University of Windsor

Scholarship at UWindsor

\title{
An Eulerian finite element model for determination of deformation state of a copper subjected to orthogonal cutting.
}

Anna G. Raczy

University of Windsor

Follow this and additional works at: https://scholar.uwindsor.ca/etd

\section{Recommended Citation}

Raczy, Anna G., "An Eulerian finite element model for determination of deformation state of a copper subjected to orthogonal cutting." (2004). Electronic Theses and Dissertations. 1003.

https://scholar.uwindsor.ca/etd/1003

This online database contains the full-text of PhD dissertations and Masters' theses of University of Windsor students from 1954 forward. These documents are made available for personal study and research purposes only, in accordance with the Canadian Copyright Act and the Creative Commons license-CC BY-NC-ND (Attribution, Non-Commercial, No Derivative Works). Under this license, works must always be attributed to the copyright holder (original author), cannot be used for any commercial purposes, and may not be altered. Any other use would require the permission of the copyright holder. Students may inquire about withdrawing their dissertation and/or thesis from this database. For additional inquiries, please contact the repository administrator via email (scholarship@uwindsor.ca) or by telephone at 519-253-3000ext. 3208. 


\title{
An Eulerian Finite Element Model for Determination of Deformation State of a Copper Subjected to Orthogonal Cutting
}

By

Anna G. Raczy

\begin{abstract}
A Thesis
Submitted to the Faculty of Graduate Studies and Research through the Engineering Materials Graduate Program in Partial Fulfillment of the Requirements for the Degree of Master of Applied Science in Engineering Materials at the University of Windsor
\end{abstract}

Windsor, Ontario, Canada

2004

(C) 2004 Anna G. Raczy 


\author{
National Library \\ of Canada \\ Acquisitions and \\ Bibliographic Services \\ 395 Wellington Street \\ Ottawa ON K1A ON4 \\ Canada
}

Bibliothèque nationale

du Canada

Acquisisitons et services bibliographiques

395, rue Wellington Ottawa ON K1A ON4 Canada
Your file Votre référence ISBN: 0-612-92521-8 Our file Notre référence ISBN: 0-612-92521-8
The author has granted a nonexclusive licence allowing the National Library of Canada to reproduce, loan, distribute or sell copies of this thesis in microform, paper or electronic formats.

The author retains ownership of the copyright in this thesis. Neither the thesis nor substantial extracts from it may be printed or otherwise reproduced without the author's permission.
L'auteur a accordé une licence non exclusive permettant à la Bibliothèque nationale du Canada de reproduire, prêter, distribuer ou vendre des copies de cette thèse sous la forme de microfiche/film, de reproduction sur papier ou sur format électronique.

L'auteur conserve la propriété du droit d'auteur qui protège cette thèse. $\mathrm{Ni}$ la thèse ni des extraits substantiels de celle-ci ne doivent être imprimés ou aturement reproduits sans son autorisation.
In compliance with the Canadian Privacy Act some supporting forms may have been removed from this dissertation.

While these forms may be included in the document page count, their removal does not represent any loss of content from the dissertation.
Conformément à la loi canadienne sur la protection de la vie privée, quelques formulaires secondaires ont été enlevés de ce manuscrit.

Bien que ces formulaires aient inclus dans la pagination, il n'y aura aucun contenu manquant.

\section{Canadä}




\begin{abstract}
The aim of the present work was to develop a finite element model for the prediction of the deformation state of copper subjected to orthogonal cutting. Numerical investigations were performed using the Lagrangian, Arbitrary Lagrangian-Eulerian (ALE), and Eulerian element formulations. The results of the Lagrangian studies showed that the element formulation has limitations in the modeling of high strain and high strain rate processes, due to accumulation of severe mesh distortion and ensuing degradation of simulation accuracy. Although adapting the Lagrangian mesh or activating ALE smoothing reduced mesh distortion, ultimately the capabilities of the Eulerian element formulation surpassed those of the Lagrangian and ALE element formulations.

The results of the Eulerian element formulation were scrutinized to a high degree, and comparisons were made to experimental results in areas of chip geometry, strain and stress distributions, force predictions, and power consumption. Material behaviour of the workpiece was studied using a Johnson-Cook constitutive relation and an elastic plastic hydrodynamic material model.

Research completed in the present work showed that the Eulerian element formulation was the most effective in modeling metal cutting, especially when the hydrodynamic material model was employed to represent the workpiece. The following correlations were drawn between the experimental findings and the numerical model employing the hydrodynamic material:
\end{abstract}

1. The maximum experimental stress $(\sigma=422 \mathrm{MPa})$ and strain $\left(\bar{\varepsilon}^{p}=8.1\right)$ were located at the tool tip and corresponded well with the numerical results, which predicted a maximum strain of 5.6 and maximum stress of $410 \mathrm{MPa}$ also at the tool tip.

2. The stresses and strains along the primary shear plane were found to decrease from a maximum at the tool tip to a minimum at the chip root, in both experimental and numerical observations.

3. An increase in strain and stress was detected beneath the machined surface and adjacent to the rake face in the numerical model. The maximum stress 
and strain in the machined surface, predicted as 3.5 and $360 \mathrm{MPa}$, respectively, were within $5 \%$ of the experimental results. The secondary deformation zone contained similar numerical stresses and strains

4. The sizes of both the primary deformation zone $(600 \mu \mathrm{m})$, and the secondary deformation zone $(80 \mu \mathrm{m})$ predicted by the hydrodynamic material model were in good agreement with the experimental observations.

5. Overall the numerical stress and strain values were within $5-25 \%$ of the experimental findings.

In summary, the finite element model best capable of predicting the deformation of copper subjected to metal cutting operations was one, which modeled the workpiece utilizing the Eulerian element formulation in combination with the elastic plastic hydrodynamic material model. Work completed in the scope of this project has established that the Eulerian element formulation can significantly contribute to the finite element modeling of high deformation processes, such as metal cutting. 
To My Parents, Halina and Zdzisław Raczy, for their endless support and encouragement 


\section{ACKNOWLEDGMENTS}

I would like to express my most sincere gratitude to Dr. Ahmet Alpas and Dr. William Altenhof for their supervision and support. Their knowledge and expertise have been of immeasurable assistance throughout my graduate and undergraduate studies.

My sincere thanks to Dr. Tom Perry and Dr. Yang-Tse Cheng, of General Motors, for their valuable discussions and suggestions; Ms. Marilyn Murphy and Ms. Barbara Denomey for their administrative assistance; Mr. John Robinson for his technical assistance; and my fellow NSERC/GM IRC researchers for their support and friendship.

The financial assistance provided by NSERC (Natural Sciences and Engineering Research Council of Canada), General Motors of Canada Limited, and OGS (The Ontario Graduate Scholarship) is gratefully acknowledged.

Finally, I would like to thank my parents, Halina and Zdzisław, and my sister, Magdalena, for the support, encouragement and patience they have given me throughout my graduate studies. 


\section{TABLE OF CONTENTS}

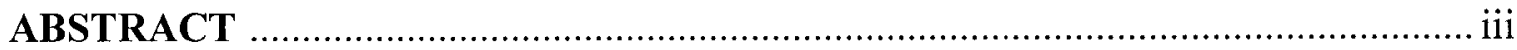

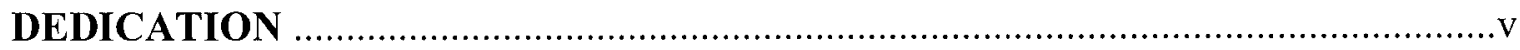

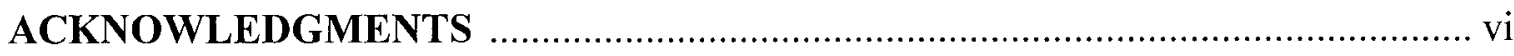

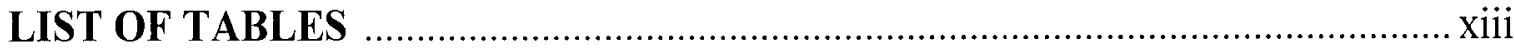

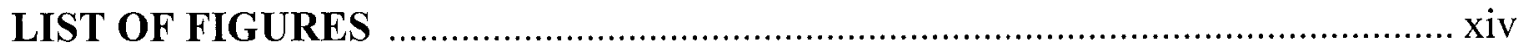

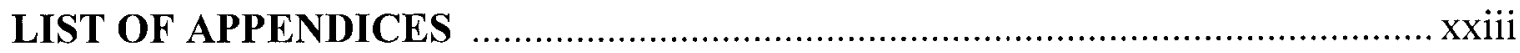

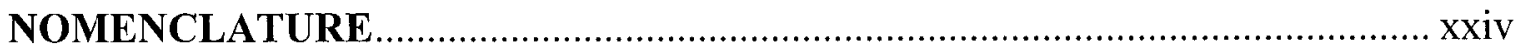

CHAPTER 1: Introduction .......................................................... 1

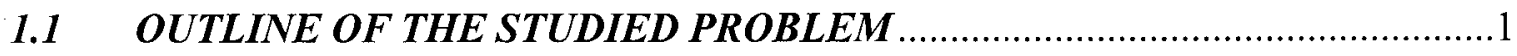

1.2 PREDICTION OF WORKPIECE BEHAVIOUR ..........................................

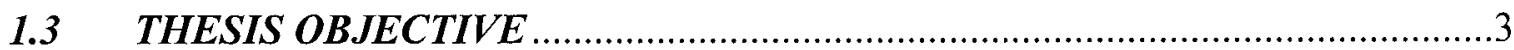

CHAPTER 2: Literature Review .................................................... 4

$2.1 \quad$ BASICS OF MACHINING OPERATIONS ........................................

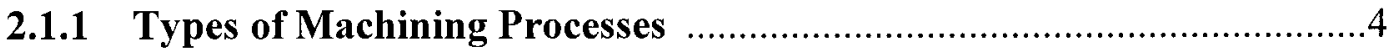

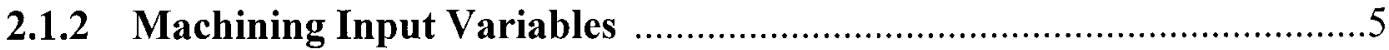

1. Workpiece Properties ............................................................5

2. Cutting Tool Properties ............................................................5

3. Cutting Parameters .............................................................

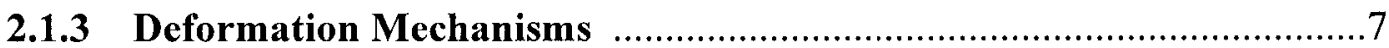

2.1.4 Theoretical Models of Metal Cutting Operations ..............................8

2.1.5 Mechanics of Chip Formation ................................................... 10

1. Force, Stress and Power Consumption ...................................10

2. Shear Plane Angle ..............................................................12

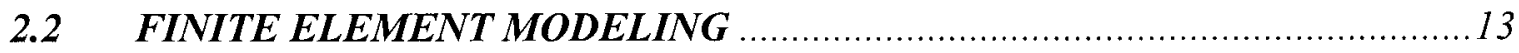

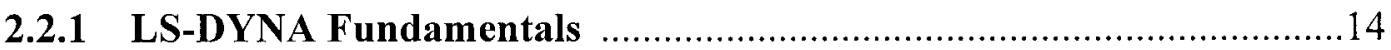

2.2.2 Eulerian, Lagrangian and ALE Element Formulations ...................17

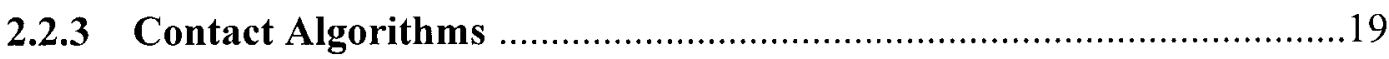

vii 


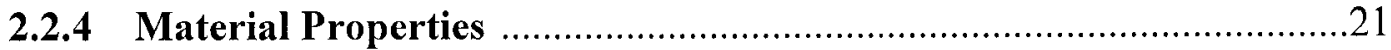

2.2.5 Adaptivity in Lagrangian Element Formulation ..........................23

2.2.6 Structure of a Finite Element Input File ........................................23

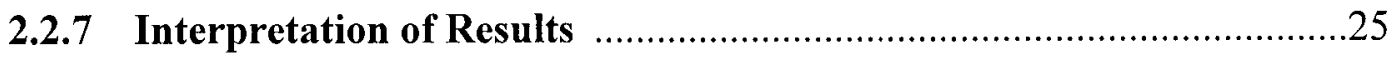

2.3 EXISTING FINITE ELEMENT MODELS OF METAL CUTTING ............28

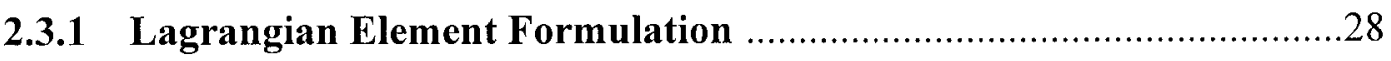

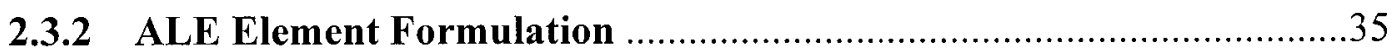

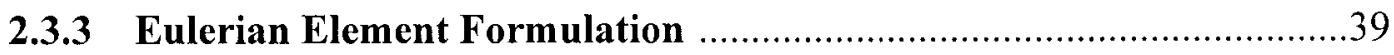

2.4 EXPERIMENTAL DATA USED FOR MODEL VERIFICATION IN THE

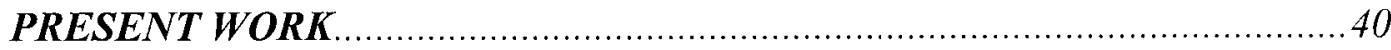

CHAPTER 3: Research Focus ........................................................ 46

\section{CHAPTER 4: Finite Element Procedures I: Metal Cutting}

Model Employing the Lagrangian Element Formulation ......... 47

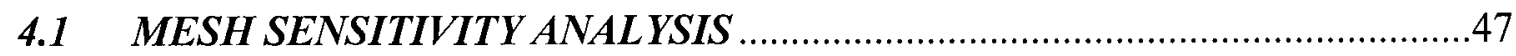

4.1.1 Geometry and Mesh Generation ................................................47

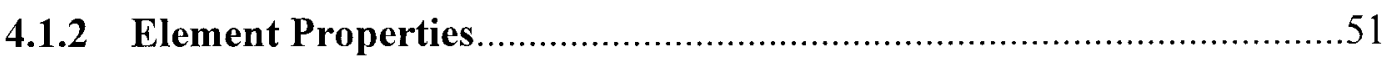

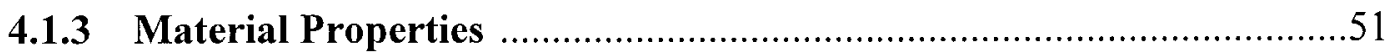

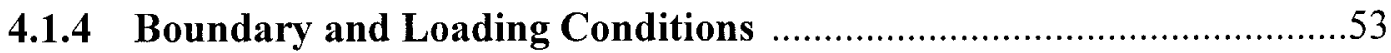

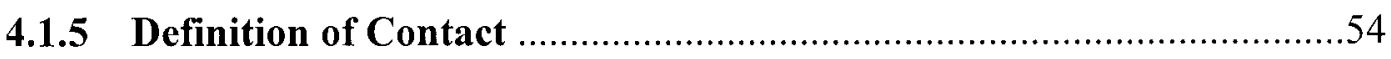

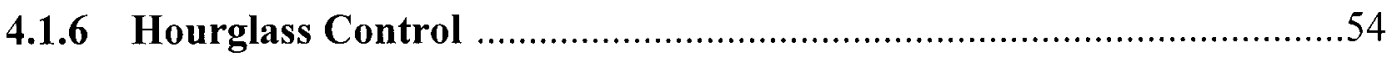

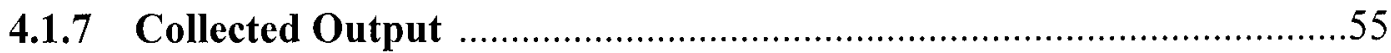

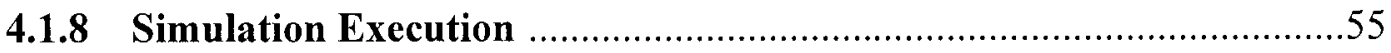

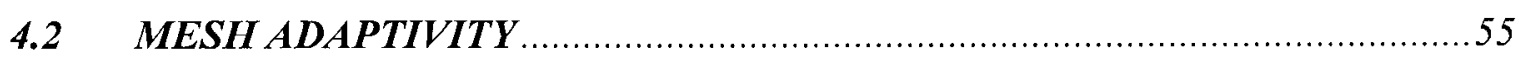

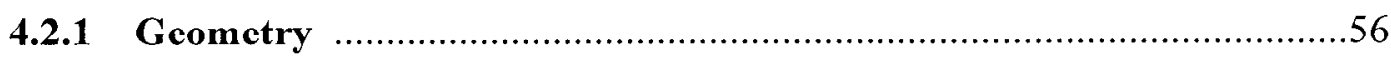

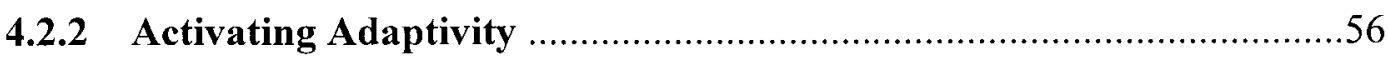




\section{CHAPTER 5: Finite Element Procedures II: Metal Cutting}

Model Employing the ALE Element Formulation .......................... 59

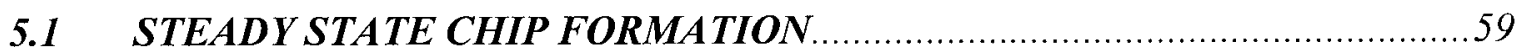

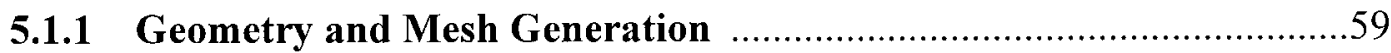

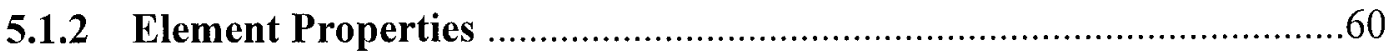

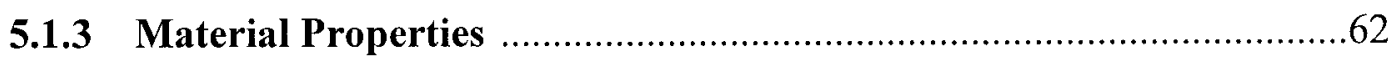

5.1.4 Applied Boundary Conditions and Motion ...................................62

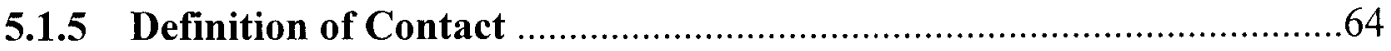

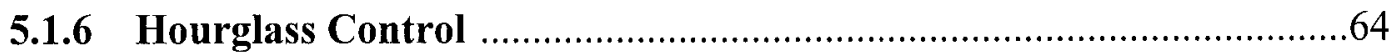

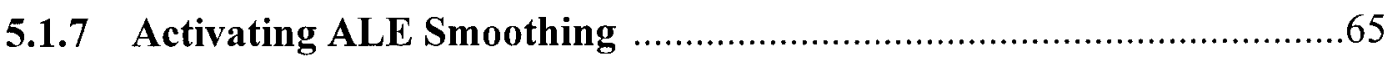

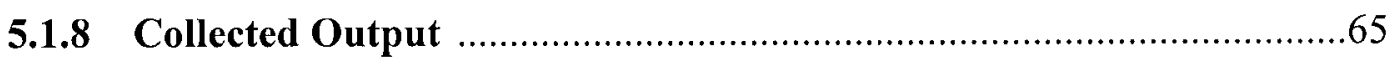

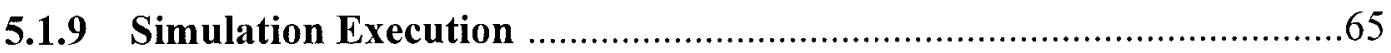

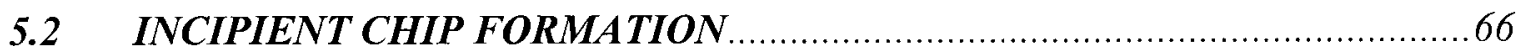

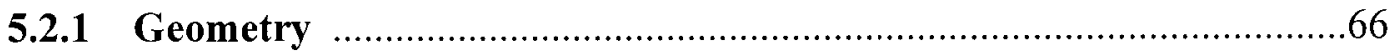

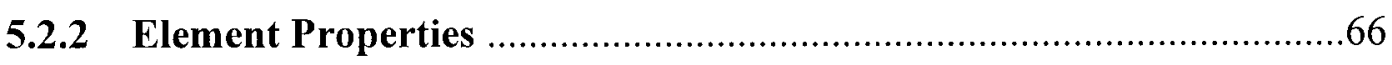

5.2.3 Applied Boundary Conditions …..............................................68

\section{CHAPTER 6: Finite Element Procedures III: Metal Cutting} Model Employing the Eulerian Element Formulation ................69

6.1 WORKPIECE MOVING INTO A STATIONARY TOOL _........................69

6.1.1 Geometry and Mesh Generation ......................................................69

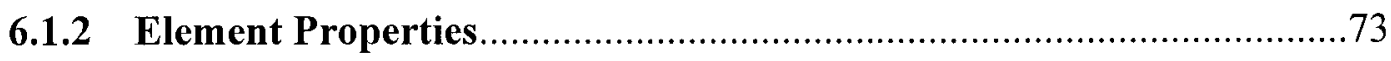

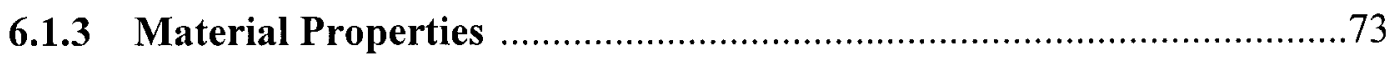

1. Johnson-Cook Constitutive Equation .....................................74

2. Hydrodynamic Material Model ...............................................76

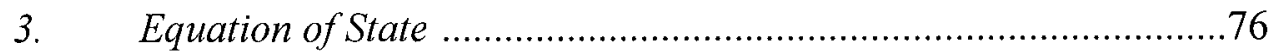

6.1.4 Applied Boundary Conditions and Workpiece Motion ......................79

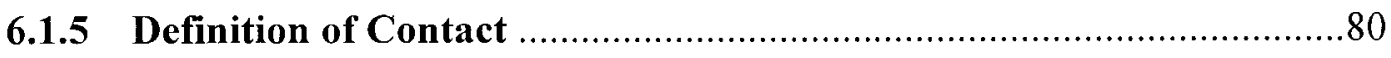

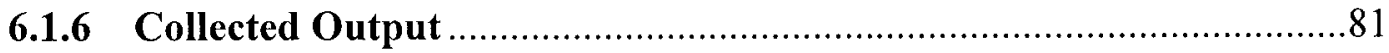

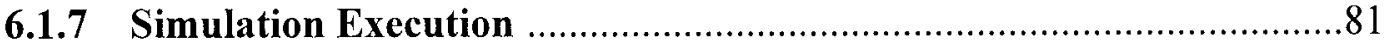


CHAPTER 7: Finite Element Results I: Metal Cutting Model Employing the Lagrangian Element Formulation ..................... 82

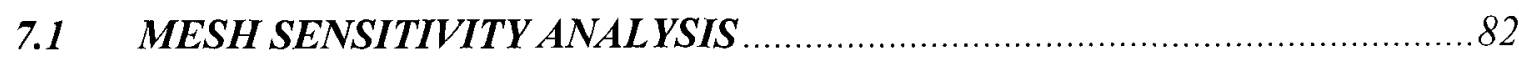

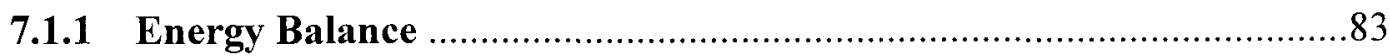

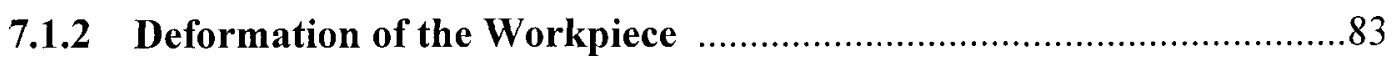

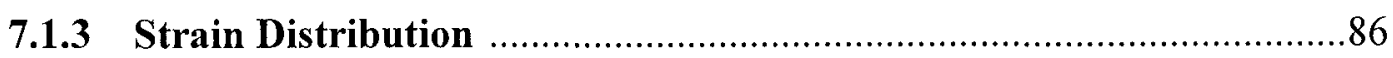

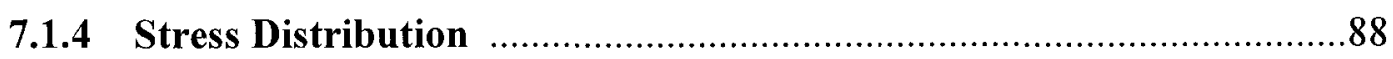

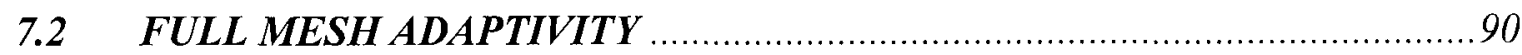

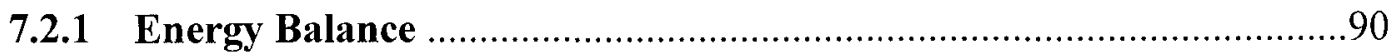

7.2.2 Deformation of the Workpiece …………………............................90

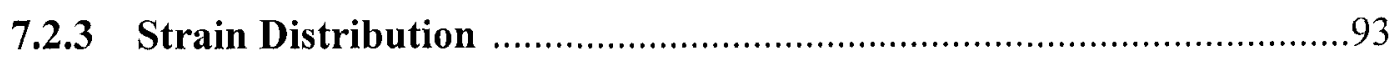

7.2.4 Stress Distribution ………………………………………………....93

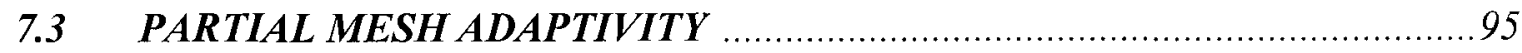

7.3.1 Deformation of the Workpiece …………………………………........95

CHAPTER 8: Finite Element Results II: Metal Cutting Model

Employing the ALE Element Formulation ............................... 97

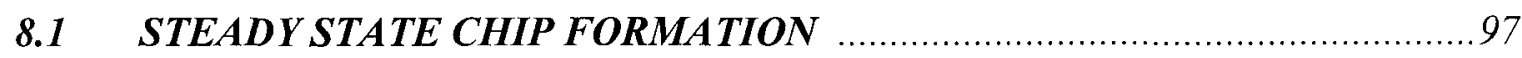

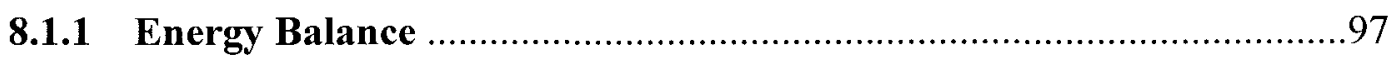

8.1.2 Deformation of the Workpiece ......................................................99

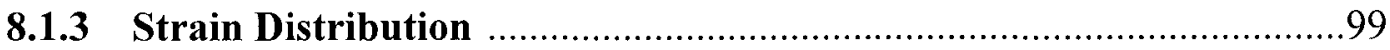

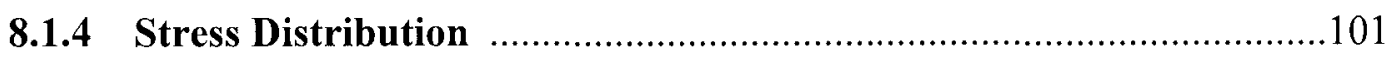

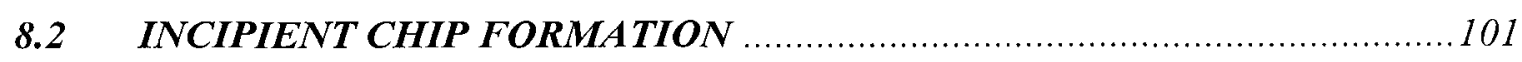

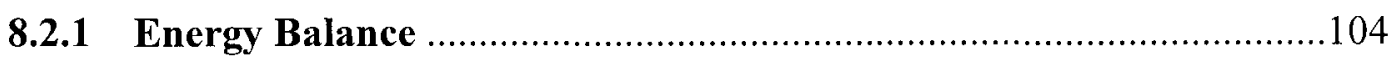

8.2.2 Deformation of the Workpiece ............................................... 104

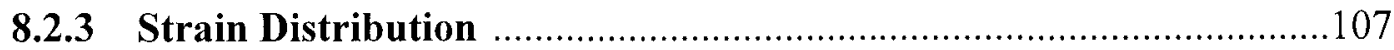

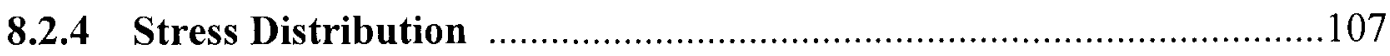




\section{CHAPTER 9: Finite Element Results III: Metal Cutting Model}

Employing the Eulerian Element Formulation ......................... 110

9.1 EFFECT OF USING ONE PLANE AND TWO PLANES OF

SYMMETRY

9.1.1 Deformation of the Workpiece …………………………………....111

9.1.2 Strain Distribution ........................................................................111

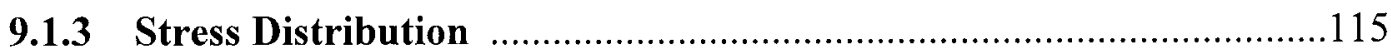

9.2 RESULTS GENERATED FROM MATERIAL MODEL $1 \ldots \ldots \ldots \ldots \ldots \ldots \ldots \ldots . .117$

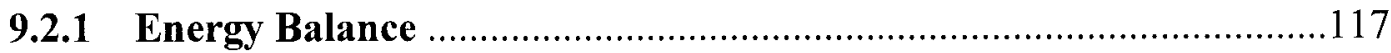

9.2.2 Deformation of the Workpiece …………………..........................119

9.2.3 Strain Distribution .........................................................................119

9.2.4 Stress Distribution …………………………...............................122

9.2.5 External Work ............................................................................122

9.2.6 Force and Power Predictions .............................................................124

9.3 RESULTS GENERATED FROM MATERIAL MODEL 2 ……................128

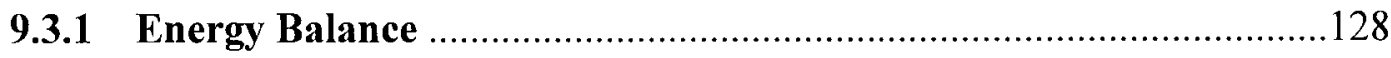

9.3.2 Deformation of the Workpiece …………………………………......128

9.3.3 Strain Distribution ...........................................................................

9.3.4 Stress Distribution ……………………………….......................130

9.3.5 External Work ………………………………............................133

9.3.6 Force and Power Predictions ...........................................................135

9.4 RESULTS GENERATED FROM MATERIAL MODEL $3 \ldots \ldots \ldots \ldots \ldots \ldots \ldots \ldots \ldots . . .138$

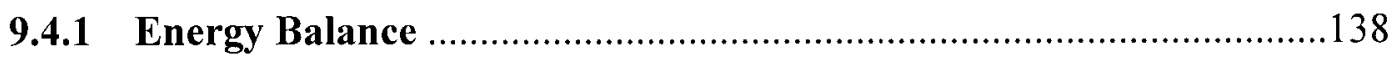

9.4.2 Deformation of the Workpiece …………………………………...138

9.4.3 Strain Distribution ..........................................................................140

9.4.4 Stress Distribution ........................................................................140

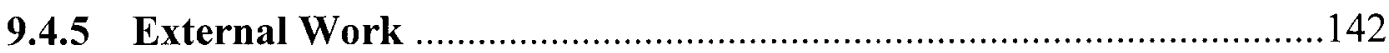

9.4.6 Force and Power Predictions ...........................................................142 
10.1 LAGRANGIAN ELEMENT FORMULATION ……….............................. 146

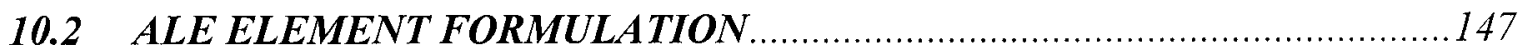

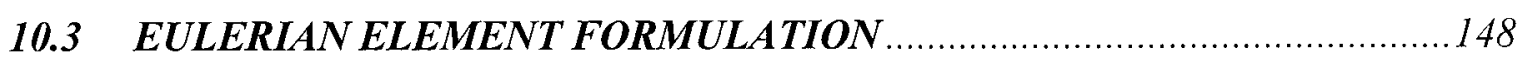

10.3.1 Deformation of the Workpiece .........................................................148

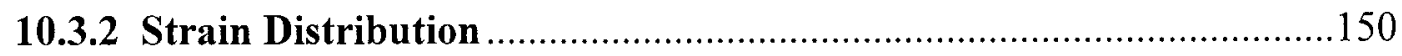

1. $\quad$ Machined Surface ................................................................150

2. Secondary Deformation Zone (SDZ) ……………………….......153

3. Primary Deformation Zone (PDZ) ...........................................154

10.3.3 Stress Distribution ........................................................................155

1. Machined Surface ...............................................................155

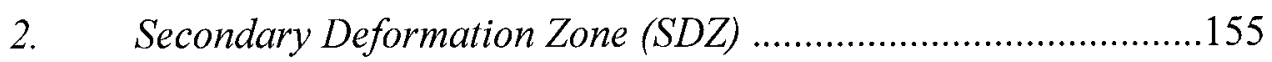

3. Primary Deformation Zone (PDZ) .............................................156

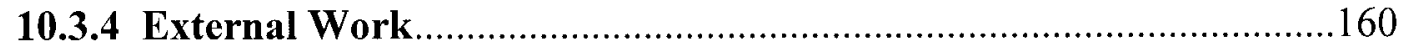

10.3.5 Forces and Power Consumption .........................................................162

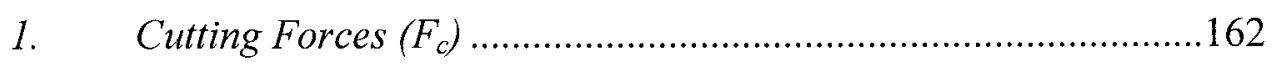

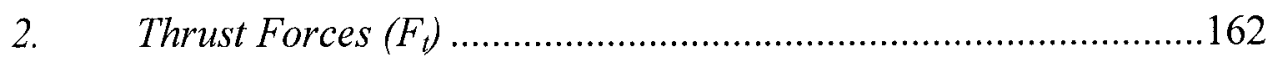

3. Total Power Consumed $\left(P_{t}\right) \ldots \ldots \ldots \ldots \ldots \ldots \ldots \ldots \ldots \ldots \ldots \ldots \ldots \ldots \ldots \ldots \ldots \ldots \ldots \ldots \ldots \ldots . . .166$

10.4 DETERMINATION OF BEST NUMERICAL MODEL ……….................

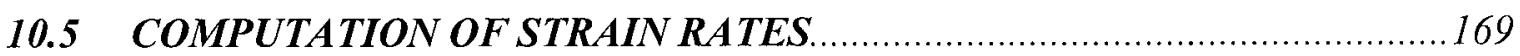

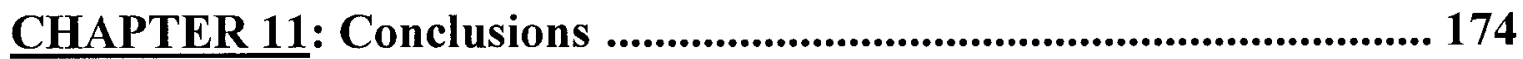

CHAPTER 12: Suggestions for Future Work ...................................... 178

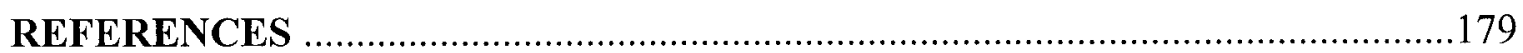

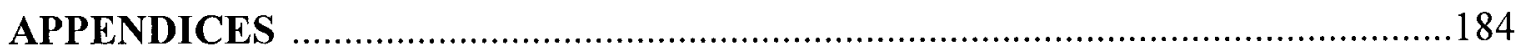

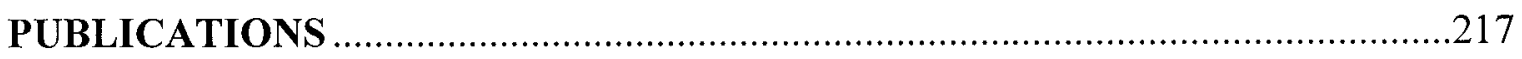

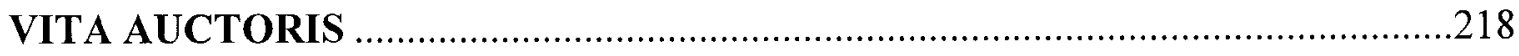

xii 


\section{LIST OF TABLES}

\section{Chapter 2:}

Table 2.1: Properties of the commercial purity copper workpiece material in the experimental machining tests, as measured experimentally and as reported in the ASM Metals Handbook: Volume 2.

Table 2.2: Properties of SiAlON grade Silicon Nitride tool inserts in the experimental machining tests, obtained from ASM Metals Handbook: Volume 16

\section{Chapter 4:}

Table 4.1: Number of elements and minimum element size in meshes (a) through (d) in the Lagrangian element formulation study

Table 4.2: $\quad$ Stress-plastic strain values used to characterize commercial purity ETP copper material behaviour for Material type 24 .

\section{Chapter 6:}

Table 6.1: Number of elements in each of the two geometries of the Eulerian element formulation study

Table 6.2: Comparison between properties of commercial purity OFHC and ETP coppers obtained from ASM Metals Handbook: Volume 2 .75

Table 6.3: Johnson-Cook parameters used in Material Models 1 and 2 .75

Table 6.4: Sixteen stress-plastic strain pairs derived from experimental results, and used to define the flow curve for commercial purity ETP copper.

Table 6.5: $\quad$ Parameters necessary to complete the EOS required by the material models of the commercial purity copper workpiece, obtained from the work of Steinberg.....

\section{Chapter 7:}

Table 7.1: $\quad$ Time required to process numerical simulations of each of the four meshes in the mesh sensitivity analysis.

Table 7.2: Values of maximum plastic strain within the workpiece material for Mesh (a) through (d)

Table 7.3: Values of maximum stress, in units of $\mathrm{MPa}$, within the workpiece material for Mesh (a) through (d) 


\section{Chapter 2:}

\section{LIST OF FIGURES}

Figure 2.1: $\quad$ Schematic diagram showing the workpiece and tool geometry during the process of orthogonal metal cutting

Figure 2.2: Schematic diagram of deformation zones and cutting parameters labelled on a cross-sectional workpiece undergoing orthogonal cutting, where $t_{c}$ is the chip thickness, $\alpha$ is the rake angle, $\gamma$ is the clearance angle, $f$ is the feed, $v_{c}$ is the cutting speed, and $\phi$ is the primary shear plane angle

Figure 2.3: Idealized model of the cutting process developed by Piispanen, where the shaded lamellar has just moved

Figure 2.4: Force diagram developed by Ernst and Merchant, with the assumption of a single shear plane

Figure 2.5: Flowchart for explicit time integration of a Lagrangian (Section 2.2.2) mesh, obtained from Belytschko et al.

Figure 2.6: Two-dimensional mesh illustrating the behaviour of: (a) Lagrangian mesh, and (b) Eulerian mesh, with respect to material deformation

Figure 2.7: Adaptivity methods in LS-DYNA: (a) two-pass adaptivity where the calculation is repeated after adaptive remeshing, and (b) one-pass adaptivity where calculation is not repeated after adaptive remeshing

Figure 2.8: (a) Hourglass modes in shell elements (note the hourglass shape of deformed elements), and (b) two of twelve possible hourglass modes in solid elements

Figure 2.9: Distributions of stress, in units $\mathrm{Pa}$, for (a) elastic-perfectly plastic material, and (b) elastic-plastic material with hardening and strain rate effects; obtained from the work of Komvopoulos and Erpenbeck

Figure 2.10: Distribution of strain in the elastic-plastic material with hardening and strain rate effects, for contact interface friction coefficients of (a) $\mu=0.0$ and (b) $\mu=0.15$; obtained from the work of Komvopoulos and Erpenbeck 
Figure 2.11: Variation of stress and strain within the machined surface for temperature being: (a) neglected, and (b) considered; obtained from the work of Lin and Lo

Figure 2.12: Temperature distribution obtained from a numerical model of AISI 4340 steel, for interface friction coefficients of: (a) $\mu=0.0$, (b) $\mu=0.2$, (c) $\mu=0.4$, and (d) $\mu=0.6$; obtained from the work of Shet and Deng

Figure 2.13: Comparison of the discontinuous chip formation in brass, predicted by (a) FE modeling, and (b) experimental tests; obtained from the work of Obikawa et al.

Figure 2.14: Mesh distribution in the workpiece and tool used in the FE modeling of Ceretti et al.

Figure 2.15: Mesh distribution in FE modeling of chip formation by Movaheddy et al.; Eulerian region located adjacent to the rake face is specified

Figure 2.16: Stress distribution corresponding to chip formation shown in Figure 2.15; obtained from the work of Movaheddy et al. .38

Figure 2.17: Optical microstructure of workpiece material ahead of the tool tip, obtained from the cross-section (AA shown in the insert), of a commercial purity copper sample, which has undergone orthogonal cutting. Cutting speed $v_{c}=0.6 \mathrm{~m} / \mathrm{sec}$, feed rate $f=0.25 \mathrm{~mm} / \mathrm{rev}$ and tube wall thickness $w=3.0 \mathrm{~mm}$ in accordance to the work of Elmadagli and Alpas

Figure 2.18: Flow lines defining deformation patterns of microstructure in Figure 2.17, constructed by Elmadagli and Alpas by outlining orientation changes in grain boundaries. The cutting line and primary shear plane are labelled as OC and OD, respectively

Figure 2.19: Microhardness distribution in the machined sample showing local microhardness values taken at intersections of a $225 \mu \mathrm{m} \times 125 \mu \mathrm{m}$ grid and lines of constant hardness contours, obtained from the work of Elmadagli and Alpas

Figure 2.20: Cumulative stress-plastic strain flow curve of copper and experimental stress and strain values corresponding to specific locations in the material ahead of the tool tip, reported by Elmadagli and Alpas 


\section{Chapter 4:}

Figure 4.1: Geometry used in the Lagrangian element formulation for (a) workpiece, and (b) tool

Figure 4.2: $\quad$ Meshes generated for each of the four models of the workpiece and tool with (a) uniform mesh, (b) tool and workpiece mesh refined in the PDZ and SDZ, (c) further mesh refinement in the PDZ and SDZ, (d) finest mesh in the PDZ and SDZ. Also noted are the coarsest and finest mesh locations

Figure 4.3: $\quad$ Stress-plastic strain behaviour of C11000 undergoing orthogonal cutting characterized by the Voce equation curve and curve fitting points used in Material type 24.

Figure 4.4: Mesh generated for the adaptivity study, including those for: (a) full workpiece adaptivity, and (b) partial workpiece adaptivity concentrated at the tool tip

\section{Chapter 5:}

Figure 5.1: Geometry of workpiece and tool used in the ALE element formulation analysis of steady state chip formation showing: (a) relevant dimensions, and (b) generated mesh

Figure 5.2: Comparison of the plastic region of the linear stress-strain approximation of Material type 3, to that of the Voce equation.....

Figure 5.3: Geometry of workpiece and tool used in the ALE element formulation analysis of incipient chip formation showing:

(a) relevant dimensions, and (b) generated mesh .67

\section{Chapter 6:}

Figure 6.1: Geometry of the Eulerian FE model including: (a) threedimensional view showing all pertinent parts and workpiece depth in millimetres, (b) geometry of workpiece modeled with one plane of symmetry reducing the workpiece depth by $50 \%$, and (c) geometry of workpiece modeled with two planes of symmetry reducing workpiece depth to $9 \%$ of original depth labelled in (a)

Figure 6.2: Cross-sectional view of the Eulerian geometry, showing the element distribution and relevant dimensions

Figure 6.3: Comparison of flow curve obtained from Material Model 1, Material Model 2 and Material Model 3 


\section{Chapter 7:}

Figure 7.1: Energy balance typical of the mesh sensitivity analysis in the Lagrangian mesh

Figure 7.2: $\quad$ Workpiece deformation and mesh distortion around the tool tip for Mesh (a) through (d)

Figure 7.3: Contours of effective plastic strain accumulated in the workpiece, observed in Mesh (a) through (d) of the Lagrangian element formulation study

Figure 7.4: Contours of stress, in units of $\mathrm{MPa}$, within the workpiece, observed in Mesh (a) through (d) of the Lagrangian element formulation study

Figure 7.5: Energy balance typical of the mesh sensitivity analysis of the Lagrangian mesh with adaptivity.

Figure 7.6: Progression of mesh/material deformation at a time of: (a) $0.3 \mathrm{~ms}$, (b) $0.325 \mathrm{~ms}$, and (c) termination time of $0.5 \mathrm{~ms}$. Mesh adaptivity was activated at $0.3 \mathrm{~ms}$

Figure 7.7: Contours of effective plastic strain, observed in the adapted workpiece

Figure 7.8: Contours of von Mises stress, in units of MPa, within the adapted workpiece

Figure 7.9: Deformation of the mesh with partial adaptivity. The adapted region has separated from the remainder of the mesh .96

\section{Chapter 8:}

Figure 8.1: $\quad$ Energy balance of the steady state chip formation simulation using the ALE element formulation .98

Figure 8.2: Deformation of the workpiece modeled with an ALE mesh at: (a) $\mathrm{t}=0.15 \mathrm{~ms}$, and (b) $\mathrm{t}=0.333 \mathrm{~ms}$

Figure 8.3: $\quad$ Strain distribution in the workpiece modeled with an ALE element formulation at: (a) $\mathrm{t}=0.15 \mathrm{~ms}$, and (b) $\mathrm{t}=0.333 \mathrm{~ms}$ 
Figure 8.4: Distribution of stress in units of $\mathrm{MPa}$, for the workpiece modeled with an ALE element formulation at: (a) $\mathrm{t}=0.15 \mathrm{~ms}$, and (b) $\mathrm{t}=0.333 \mathrm{~ms}$

Figure 8.5: Energy balance of the incipient state chip formation simulation using the ALE element formulation

Figure 8.6: Deformation of the workpiece modeled with an ALE mesh at:

(a) $\mathrm{t}=0.25 \mathrm{~ms}$, and $\mathrm{t}=0.5 \mathrm{~ms}$

Figure 8.7: Strain distribution in the workpiece modeled with an ALE element formulation at: $\mathrm{t}=0.25 \mathrm{~ms}$, and (b) $\mathrm{t}=0.5 \mathrm{~ms}$

Figure 8.8: Distribution of stress in units of $\mathrm{MPa}$, for the workpiece modeled with an ALE element formulation at: (a) $t=0.25 \mathrm{~ms}$, and (b) $\mathrm{t}=0.5 \mathrm{~ms}$

\section{Chapter 9:}

Figure 9.1: Chip formation for models with one and two plane(s) of symmetry at times of: (a) $7.5 \mathrm{~ms}$, (b) $10.0 \mathrm{~ms}$, and (c) $15.0 \mathrm{~ms}$.

Figure 9.2: Comparison of cross-sectional chip geometry generated by models with one and two plane(s) of symmetry, at times of: (a) $7.5 \mathrm{~ms}$, (b) $10.0 \mathrm{~ms}$, and (c) $15.0 \mathrm{~ms}$

Figure 9.3: Comparison of strain distributions in models with one and two plane(s) of symmetry, at times of: (a) $7.5 \mathrm{~ms}$, (b) $10.0 \mathrm{~ms}$, and (c) $15.0 \mathrm{~ms}$

Figure 9.4: Comparison of stress distributions in units of $\mathrm{kPa}$, for models with one and two plane(s) of symmetry, at times of: (a) $7.5 \mathrm{~ms}$, (b) $10.0 \mathrm{~ms}$, and (c) $15.0 \mathrm{~ms}$

Figure 9.5: Energy balance typical of the metal cutting simulated in the Eulerian element formulation study, with workpiece modeled as Material Model 1

Figure 9.6: Investigation of friction coefficient effect on chip thickness for Material Model 1, shown are: (a) chip geometry with corresponding coefficients of friction, and (b) coefficient of friction vs. chip thickness plot, with experimental data noted

Figure 9.7: Strain distribution in the material ahead of the tool tip in the Eulerian element formulation study, with workpiece modeled as Material Model 1 and $\mu=0.84$ 
Figure 9.8: Stress distribution in the material ahead of the tool tip, in units of $\mathrm{kPa}$, in the Eulerian element formulation study with workpiece modeled as Material Model 1 and $\mu=0.84$

Figure 9.9: Surface plot of external work variation, in units of $\mathrm{J} \cdot \mathrm{mm}^{-1}$, with respect to time and friction, in the Eulerian element formulation study with workpiece modeled as Material Model 1

Figure 9.10: Surface plot of cutting force $\left(F_{c}\right)$ variation in units of $\mathrm{N}$, with respect to time and friction, in the Eulerian element formulation study with workpiece modeled as Material Model 1

Figure 9.11: Surface plot of the thrust force $\left(F_{t}\right)$ variation in units of $\mathrm{N}$, with respect to time and friction, in the Eulerian element formulation study with workpiece modeled as Material Model 1

Figure 9.12: Surface plot of total power $\left(P_{t}\right)$ consumed, in units of $\mathrm{W}$, for metal cutting simulations in the Eulerian element formulation study, with workpiece modeled as Material Model 1

Figure 9.13: Energy balance typical of the metal cutting simulated in the Eulerian element formulation study, with workpiece modeled as Material Model 2

Figure 9.14: Investigation of friction coefficient effect on chip thickness for Material Model 2, shown are: (a) chip geometry with corresponding coefficients of friction, and (b) coefficient of friction vs. chip thickness plot, with experimental data noted

Figure 9.15: Strain distribution in the material ahead of the tool tip in the Eulerian element formulation study, with workpiece modeled as Material Model 2 and $\mu=0.39$

Figure 9.16: Stress distribution in the material ahead of the tool tip, in units of $\mathrm{kPa}$, in the Eulerian element formulation study, with workpiece modeled as Material Model 2 and $\mu=0.39$

Figure 9.17: Surface plot of external work variation, in units of $\mathrm{J} \cdot \mathrm{mm}^{-1}$, with respect to time and friction, in the Eulerian element formulation study with workpiece modeled as Material Model 2

Figure 9.18: Surface plot of cutting force $\left(F_{c}\right)$ variation, in units of $\mathrm{N}$, with respect to time and friction, in the Eulerian element formulation study with workpiece modeled as Material Model 2 
Figure 9.19: Surface plot of the thrust force $\left(F_{t}\right)$ variation in units of $\mathrm{N}$, with respect to time and friction, in the Eulerian element formulation study with workpiece modeled as Material Model 2

Figure 9.20: Surface plot of total power $\left(P_{t}\right)$ consumed, in units of $\mathrm{W}$, for metal cutting simulations in the Eulerian element formulation study with workpiece modeled as Material Model 2

Figure 9.21: Energy balance typical of the metal cutting simulated in the Eulerian element formulation study, with workpiece modeled as Material Model 3

Figure 9.22: Geometry of the chip formed by the simulation in the Eulerian element formulation study with workpiece modeled as Material Model 3

Figure 9.23: Strain distribution in the material ahead of the tool tip in the Eulerian element formulation study with workpiece modeled as Material Model 3

Figure 9.24: Stress distribution in the material ahead of the tool tip, in units of $\mathrm{kPa}$, in the Eulerian element formulation study with workpiece modeled as Material Model 3

Figure 9.25: External work variation, in units of $\mathrm{J} \cdot \mathrm{mm}^{-1}$, in the Eulerian element formulation study with workpiece modeled as Material Model 3

Figure 9.26: Cutting $\left(F_{c}\right)$, thrust $\left(F_{t}\right)$ and radial $\left(F_{r}\right)$ forces, in units of $\mathrm{N}$, in the Eulerian element formulation study with workpiece modeled as Material Model 3

Figure 9.27: Total power $\left(P_{t}\right)$ consumed, in units of $\mathrm{W}$, for the metal cutting simulation in the Eulerian element formulation study with workpiece modeled as Material Model 3

\section{Chapter 10:}

Figure 10.1: Chip geometry formed during simulation of: (a) Material Model 1 with $\mu=0.84$ at termination time of $\mathrm{t}=20.0 \mathrm{~ms}$, (b) Material Model 2 with $\mu=0.39$ at termination time of $\mathrm{t}=20.0 \mathrm{~ms}$, and (c) Material Model 3 at termination time of $\mathrm{t}=30.0 \mathrm{~ms}$

Figure 10.2: Contours of equivalent strain according to Material Model 1 with $\mu=0.84$ at $\mathrm{t}=20.0 \mathrm{~ms}$ 
Figure 10.3: Contours of equivalent strain according to Material Model 2 with $\mu=0.39$ at $\mathrm{t}=20.0 \mathrm{~ms}$

Figure 10.4: Contours of equivalent strain according to Material Model 3 at $\mathrm{t}=30.0 \mathrm{~ms}$

Figure 10.5: Contours of equivalent strain according to experimental measurements

Figure 10.6: Contours of flow stress, in units of $\mathrm{MPa}$, according to Material Model 1 with $\mu=0.84$ at $\mathrm{t}=20.0 \mathrm{~ms}$

Figure 10.7: Contours of flow stress, in units of $\mathrm{MPa}$, according to Material Model 2 with $\mu=0.39$ at $\mathrm{t}=20.0 \mathrm{~ms}$

Figure 10.8: Contours of flow stress, in units of $\mathrm{MPa}$, according to Material Model 3 at $\mathrm{t}=30.0 \mathrm{~ms}$......

Figure 10.9: Contours of flow stress, in units of $\mathrm{MPa}$, according to experimental data

Figure 10.10: External work variation, in units of $\mathrm{J} \cdot \mathrm{mm}^{-1}$ in: Material Model 1 with $\mu=0.84$ and termination time of $20.0 \mathrm{~ms}$, Material Model 2 with $\mu=0.39$ and termination time of $20.0 \mathrm{~ms}$, and Material Model 3 with termination time of $30.0 \mathrm{~ms}$

Figure 10.11: Cutting force $\left(F_{c}\right)$, in units of $\mathrm{N}$, for all three studied material models with respect to time, and the steady state cutting force for the machining tests

Figure 10.12: Thrust force $\left(F_{t}\right)$, in units of $\mathrm{N}$, for all three studied material models with respect to time, and the steady state thrust force for the machining tests

Figure 10.13: Total power $\left(P_{t}\right)$, in units of $\mathrm{W}$, for all three studied material models with respect to time, and the power consumed for the steady state of the machining tests

Figure 10.14: Variables defined in the experimental analysis of strain rate distributions in the material ahead of the tool tip

Figure 10.15: Strain rates, in units of $\mathrm{s}^{-1}$, according to (a) experimental findings, and (b) FE results with workpiece modeled by Material Model 3 


\section{Appendices:}

Figure A.1: Axially loaded member.

Figure A.2: Free body diagram of the section taken through member in Figure A.1

Figure A.3: Plot of experimental equivalent strain vs. flow stress

216

Figure A.4: Log-Log plot of the experimental stress-strain data, with a linear curve fit 


\section{LIST OF APPENDICES}

APPENDIX A: $\quad$ Example Problem: Analysis of a Link Element ............................184

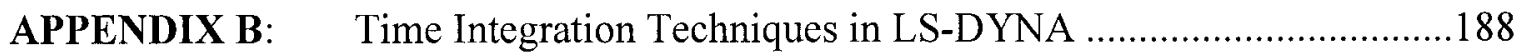

APPENDIX C: Sample LS-DYNA input for Lagrangian Element Formulation Model ............................................................191

APPENDIX D: Sample LS-DYNA input for Lagrangian Element Formulation Model with Full Adaptivity....................................194

APPENDIX E: Sample LS-DYNA input for Lagrangian Element Formulation Model with Partial Adaptivity

APPENDIX F: $\quad$ Sample LS-DYNA input for ALE Element Formulation Model for Steady State Chip Formation 200

APPENDIX G: Sample LS-DYNA input for ALE Element Formulation Model for Incipient Chip Formation. .203

APPENDIX H: Sample LS-DYNA input for Eulerian Element Formulation Study with Material Model 1 206

APPENDIX I: $\quad$ Sample LS-DYNA input for Eulerian Element Formulation Study with Material Model 2

APPENDIX J: $\quad$ Sample LS-DYNA input for Eulerian Element Formulation Study with Material Model 3

APPENDIX K: Derivation of Johnson-Cook Parameters from experimental results 


\section{NOMENCLATURE}

$\alpha \quad$ Rake angle: angle between the rake face of the tool and the direction perpendicular to the cutting direction, in degrees $\left(^{\circ}\right)$

$\gamma$

$t_{c}$

$v_{c}$

$F_{c}$

$F_{t}$

$F_{s}$

$N_{s}$

$F_{f}$

$N_{f}$

$\mu$
Clearance Angle: angle between the clearance face of the tool and the cutting direction, in degrees $\left(^{\circ}\right)$

Chip Thickness: distance between the tool rake face and the free surface of the chip, measured perpendicular to the rake face of the tool, in millimetres $(\mathrm{mm})$

Feed: the cutting depth per one cutting edge and revolution, in millimetres (mm)

Cutting speed: surface speed of the lathe rotation in a turning operation in units of $\left(\mathrm{mm} \cdot \mathrm{s}^{-1}\right)$

Primary Shear Plane Angle: angle between the cutting direction and the primary shear plane, in degrees $\left({ }^{\circ}\right)$

Depth of Cut: distance perpendicular to cutting direction, between the surface machined in the previous tool pass and the surface exposed in the current tool pass, in millimetres (mm)

Cutting Force: force measured along the cutting direction, in Newtons (N)

Tangent Force: force measured along a direction perpendicular to the cutting direction, in units of Newtons $(\mathrm{N})$

Shear Force: force measured along the primary shear plane, in units of Newtons $(\mathrm{N})$

Normal Shear Force: force measured along a direction perpendicular to the primary shear plane, in units of Newtons (N)

Friction Force: force measured along the rake face of the tool, in units of Newtons (N)

Normal Friction Force: force measured along a direction perpendicular to the rake face, in units of Newtons $(\mathrm{N})$

Coefficient of Friction: coefficient of friction between the tool and the workpiece, dimensionless 
$\sigma_{s}$

$\tau_{s}$

w

$\sigma_{f}$

$\tau_{f}$

$\sigma_{\max }$

$x_{c}$

$l_{c} \quad$ Contact Length: length of the contact between the chip and tool rake face, in units of millimetres $(\mathrm{mm})$

a Parameter used in calculations of $\sigma_{f}$, dependent on material properties, dimensionless

$\tau_{y} \quad$ Yield Shear Stress: shear stress at which yielding of the material occurs in units of Pascals $\times 10^{6}(\mathrm{MPa})$

$P_{t} \quad$ Total Power: power consumed during the metal cutting process, in units of Watts (W)

$\beta \quad$ Angle associated with the resultant of the shear and normal forces on the rake face, in degrees $\left(^{\circ}\right)$

$\delta W \quad$ Virtual Work: in units of Jules (J)

[M] Mass Matrix: matrix containing information on nodal mass distribution in a discretized body of a FE analysis, in units of kilograms $(\mathrm{kg})$

[C] Damping Matrix: matrix containing information on the degree of damping applied to nodes of a discretized body of a FE analysis, in units of $\left(\mathrm{kg} \cdot \mathrm{s}^{-1}\right)$

[K] Stiffness Matrix: matrix containing information on the stiffness applied to nodes of a discretized body of a FE analysis, in units of $\left(\mathrm{kg} \cdot \mathrm{s}^{-2}\right)$ 
$\left\{F^{e x t}\right\} \quad$ External Force Vector: Vector summarizing the external forces being applied on each node of a discretized body, in units of Newtons $(\mathrm{N})$

$\{i i\} \quad$ Nodal acceleration Vector: Vector summarizing the accelerations of all nodes of a discretized body, in units of $\left(\mathrm{mm} \cdot \mathrm{s}^{-2}\right)$

$\{\dot{u}\} \quad$ Nodal Velocity Vector: Vector summarizing the velocities of all nodes of a discretized body, in units of $\left(\mathrm{mm} \cdot \mathrm{s}^{-1}\right)$

$\{u\} \quad$ Nodal Displacement Vector: Vector summarizing the displacements of all nodes of a discretized body, in units of millimetres ( $\mathrm{mm}$ )

$\left\{F^{i n t}\right\} \quad$ Internal Force Vector: Vector summarizing the internal forces of the discretized body resulting from stress and strain fields, in units of Newtons (N)

$\varepsilon$

$\dot{\varepsilon}$

$t$

$\Delta t$

$t_{\text {termination }}$

$C_{\text {user }}$

$E$

$v$

$A_{c}$

$K$

$\tau$

$\sigma_{n}$

$\mu_{d}$
Strain: measure of deformation in material, dimensionless

Strain Rate: rate of deformation in material, in units of $\left(\mathrm{s}^{-1}\right)$

Time: time measured in units of seconds (s)

Time Interval: Length of one time step in a FE simulation, in units of seconds (s)

Termination Time: time at which a FE simulation is to terminate, in units of seconds (s)

User defined scale factor for the calculation of the stiffness of springs in $\mathrm{FE}$ contact, dimensionless

Young 's Modulus: material constant in units of Pascals $\times 10^{6}(\mathrm{MPa})$

Poisson's Ratio: material constant, dimensionless

Area: face area of the element in contact, in units of $\left(\mathrm{mm}^{2}\right)$

Bulk Modulus: material constant, in units of Pascals $\times 10^{6}(\mathrm{MPa})$

Shear Interface Stress: stress measured along the contact interface, in units of Pascals $\times 10^{6}(\mathrm{MPa})$

Normal Interface Stress: stress measured perpendicular to the contact interface, in units of Pascals $\times 10^{6}(\mathrm{MPa})$

Dynamic Coefficient of Friction: dimensionless 


\begin{tabular}{|c|c|}
\hline$\mu_{S}$ & Static Coefficient of Friction: dimensionless \\
\hline$D C$ & $\begin{array}{l}\text { Decay Coefficient: user defined parameter, which controls the gradient of } \\
\text { the transition from static to dynamic coefficient of friction during contact, } \\
\text { dimensionless }\end{array}$ \\
\hline$v_{\text {rel }}$ & $\begin{array}{l}\text { Relative Velocity: relative velocity between two entities in contact, in units } \\
\text { of }\left(\mathrm{mm} \cdot \mathrm{s}^{-2}\right)\end{array}$ \\
\hline$\sigma_{y}$ & $\begin{array}{l}\text { Yield Strength: Stress at which a particular material undergoes yielding, in } \\
\text { units of Pascals } \times 10^{6}(\mathrm{MPa})\end{array}$ \\
\hline$n s n$ & $\begin{array}{l}\text { Number of Slave Nodes: parameter used as a counter during summation of } \\
\text { interface forces over all slave nodes in a discretized body, dimensionless }\end{array}$ \\
\hline$n m n$ & $\begin{array}{l}\text { Number of Master Nodes: parameter used as a counter during summation of } \\
\text { interface forces over all master nodes in a discretized body, dimensionless }\end{array}$ \\
\hline$\Delta F_{i}^{\text {slave }}$ & $\begin{array}{l}\text { Slave Interface Force: force at the contact interface between the slave and } \\
\text { master entities, in units of Newtons, }(\mathrm{N})\end{array}$ \\
\hline$\Delta F_{i}^{\text {master }}$ & $\begin{array}{l}\text { Master Interface Force: force at the contact interface between the slave and } \\
\text { master entities, in units of Newtons, (N) }\end{array}$ \\
\hline$\Delta d_{i}^{\text {slave }}$ & $\begin{array}{l}\text { Slave Distance: incremental distance that the slave node has moved during a } \\
\text { time step, in units of millimetres, }(\mathrm{mm})\end{array}$ \\
\hline$\Delta d_{i}^{\text {master }}$ & $\begin{array}{l}\text { Master Distance: incremental distance that the master node has moved } \\
\text { during a time step, in units of millimetres, }(\mathrm{mm})\end{array}$ \\
\hline$E_{\text {contact }}$ & $\begin{array}{l}\text { Contact Energy: Energy arising from the forces generated during contact } \\
\text { between two entities, in units of Jules }(\mathrm{J})\end{array}$ \\
\hline$\rho$ & Density: material constant, in units of $\left(\mathrm{Mg} \cdot \mathrm{cm}^{-3}\right)$ \\
\hline$G$ & Shear Modulus: material constant, in units of Pascals $\times 10^{6}(\mathrm{MPa})$ \\
\hline$T_{\text {melt }}$ & $\begin{array}{l}\text { Melting Temperature: Temperature at which a particular material transitions } \\
\text { from a solid to liquid state, in units of degree Celcius }\left({ }^{\circ} \mathrm{C}\right)\end{array}$ \\
\hline$C_{p}$ & Specific Heat Capacity: in units of $\left(\mathrm{J} \cdot \mathrm{kg}^{-1} \cdot{ }^{\circ} \mathrm{C}^{-1}\right)$ \\
\hline$H$ & Hardness: hardness of a material, in units of Pascals $\times 10^{6}(\mathrm{MPa})$ \\
\hline $\bar{\varepsilon}^{p}$ or $\varepsilon^{p}$ & Effective Plastic Strain: Measure of permanent deformation, dimensionless \\
\hline
\end{tabular}


$\theta \quad$ Deformation angle: calculated from the shear plane angle and equivalent to $90^{\circ}-\phi$, in units of degrees $\left(^{\circ}\right)$

$C$ Constraint factor relating flow stress to hardness $(C=3.0$ for Knoop indenter), dimensionless

$\sigma$

Flow Stress: flow stress accumulated in a material, in units of Pascals $\times 10^{6}(\mathrm{MPa})$

$\sigma_{\text {sat }} \quad$ Saturation Stress: stress at which the work hardening becomes zero, in units of Pascals $\times 10^{6}(\mathrm{MPa})$

$\sigma_{0} \quad$ Yield Stress: value of flow stress at zero plastic strain, in units of Pascals $\times 10^{6}(\mathrm{MPa})$

$\varepsilon_{c} \quad$ Material constant necessary to complete the Voce Equation, dimensionless

$E_{t} \quad$ Tangent Elastic Modulus: Elastic modulus defining the stress-strain slope in the plastic region, in units of Pascals $\times 10^{6}(\mathrm{MPa})$

A Johnson-Cook parameter: equivalent to the yield stress of the material, in units of Pascals $\times 10^{6}(\mathrm{MPa})$

B Johnson-Cook parameter: in units of Pascals $\times 10^{6}(\mathrm{MPa})$

n Johnson-Cook parameter: work hardening exponent, dimensionless

C Johnson-Cook parameter: determines magnitude of strain rate effects, dimensionless

$m$ Johnson-Cook parameter: determines magnitude of temperature effects, dimensionless

$\dot{\varepsilon}^{*} \quad$ Normalized Equivalent Plastic Strain Rate: in units of $\left(\mathrm{s}^{-1}\right)$

$T^{*} \quad$ Homologous Temperature: dimensionless

$T_{\text {room }} \quad$ Room Temperature: in units of degree Celsius $\left({ }^{\circ} \mathrm{C}\right)$

$T \quad$ Temperature in material during simulation, in units of degree Celsius $\left({ }^{\circ} \mathrm{C}\right)$

$E_{v} \quad$ Energy per Initial Volume: Energy initially stored in a designated volume, in units of $\left(\mathrm{J} \cdot \mathrm{m}^{-3}\right)$

$\rho_{0} \quad$ Initial Density: in units of $\left(\mathrm{Mg} \cdot \mathrm{cm}^{-3}\right)$ 
$C_{o} \quad$ Bulk Sound Speed: material constant in units of $\left(\mathrm{mm} \cdot \mathrm{s}^{-1}\right)$

$S_{1}, S_{2}, S_{3} \quad$ Linear, quadratic, and cubic coefficients, respectively, relating material shock velocity to particle velocity, dimensionless

$\gamma_{o} \quad$ Grüneisen gamma: material constant, dimensionless

$\xi \quad$ Measure of compression, dimensionless

$b \quad$ Coefficient of volume dependence of the Grüneisen gamma, dimensionless

$d \varepsilon$ or $\partial \varepsilon \quad$ Strain increment: dimensionless

$d t$ or $\partial t \quad$ Time increment: in units of seconds (s)

$v_{c h i p} \quad$ Chip velocity: in units of $\left(\mathrm{mm} \cdot \mathrm{s}^{-1}\right)$

xxix 


\section{CHAPTER 1}

\section{Introduction}

\subsection{OUTLINE OF THE STUDIED PROBLEM}

Machining is the process of removing unwanted material from a workpiece; turning is a type of machining process, in which the removal of material is done by a single-point cutting tool. Cutting fluids have frequently been utilized in the machining process to increase tool life and improve surface finish. Due to increasing government demands for environmentally safe handling and disposal of cutting fluids, the cost of their use has risen significantly thereby motivating industry to shift the focus to a dry machining process. This process is made difficult due to high temperatures occurring within the workpiece, and caused by plastic deformation. Hence the study of machining can be reduced to the analysis of plastic deformation during chip formation. Deformation occurs in three regions, the primary deformation zone (PDZ), the secondary deformation zone (SDZ), and the machined surface.

\subsection{PREDICTION OF WORKPIECE BEHAVIOUR}

Many theories of machining have attempted to relate the workpiece deformation to cutting parameters including, cutting speed, rake angle, clearance angle, and feed. Despite advances made in optimizing the cutting process, through both experimental and theoretical approaches, the infinite number of input variables and the complexity of material deformation, makes the outcome of the process difficult to foresee. An experimental analysis is effective in determining important outcomes once the process is completed, but may prove to be time consuming when done on an iterative basis during the process design stage. With its capability to accurately predict material behaviour under loading, Finite Element (FE) modeling has become an increasingly sought solution to problems in the design of machining processes. 
An accurate FE model can serve to predict the behaviour of the workpiece material under designated cutting conditions. The stress and strain distributions give insight into the surface finish quality, while the cutting force predictions can estimate the power required to perform the operation. A numerical model enables the testing of various cutting parameters to forecast the ability of the process to fulfill functional requirement, while maintaining efficiency. Ultimately, the cutting parameters can be optimized to produce a reliable process while minimizing trial machining runs.

The majority of research dealing with FE modeling of metal cutting has utilized a Lagrangian $\mathrm{FE}$ formulation. In the Lagrangian element formulation the material points and element nodal points are coincident, therefore deformation of the workpiece is accompanied by the deformation of the mesh. Since large workpiece deformation is required to displace the material from the tool tip along the primary shear plane to form a chip, a Lagrangian mesh undergoes severe distortion, which can lead to a potential error. Common techniques utilized to prevent mesh distortion and decrease the magnitude of computational error involve the use of mesh adaptivity or application of a failure criterion along a pre-determined line lying parallel to the cutting direction at the level of the tool tip. The use of mesh adaptivity can be costly in computational time and yet may not aid in decreasing error if not applied appropriately. Use of a failure criterion requires the user to establish a pre-determined line along which neighbouring elements separate according to a geometrical and/or physical criterion. Once a geometrical criterion is met, separation occurs at a critical distance from the tool tip, while the physical criterion activates element separation when a critical shear stress is reached. Application of this approach approximates material fracture rather then the actual behaviour of material deformation, which is not an accurate representation of the process.

An Eulerian mesh is detached from the material and is stationary in space, so material deformation is not restricted by the mesh. Although the Eulerian formulation has remained unexplored in documented FE models of metal cutting, it provides two significant advantages: 1) the high strains observed in the machining process can be simulated without application of mesh adaptivity and without occurrence of severe mesh distortion leading to computational error, and 2) the use of an Eulerian FE formulation eliminates the need for any element separation criterion. 


\subsection{THESIS OBJECTIVE}

The objective of the present work has been to develop a Finite Element (FE) model predicting the behaviour of a material undergoing orthogonal metal cutting. Experimental investigations were paired with a numerical model to investigate the dry machining of commercial purity Electrolytic Tough Pitch (ETP) Copper. The experimental work was based on metallographic studies, from which effective plastic strains were calculated on the basis of shear angle measurements, and local flow stress values were derived from microhardness measurements. Numerical analyses were completed using the LS-DYNA explicit non-linear finite element code. Geometry and material properties of the tool and workpiece, were defined to best correspond to the experimental observations. Correlation of numerical results to experimental findings with respect to chip geometry, distribution of effective plastic strain, and flow stress, allowed for continuous model verification throughout its development.

The Lagrangian, ALE, and Eulerian element formulations have each been studied, to determine which is the most effective in modeling of workpiece displacement, or chip formation, during a machining operation. The most efficient element formulation has been scrutinized through experimental-numerical correlation to validate model accuracy in predicting material deformation, stress distribution, strain distribution, cutting and thrust forces, and power consumption. With the support of the numerical results, a procedure was also developed for calculation of strain rates and their distributions in the material ahead of the tool tip. 


\section{CHAPTER 2}

\section{Literature Review}

The understanding of microstructural aspects of deformation behaviour of materials subjected to machining operations is crucial to the successful development of a FE model for metal cutting. To gain the knowledge required to build a precise FE model, information was collected on the basics of metal cutting processes and FE methods, and is presented in this chapter. A summary of previous attempts made in modeling of metal cutting is also included in this chapter. Past research originally provided a strong foundation for the building of the presented FE model, but has also demonstrated its truly innovative nature. Lastly, results obtained from an experimental investigation on machined chips are presented. These observations were used in establishing the validity of the model through numerical-experimental correlation.

\subsection{BASICS OF MACHINING OPERATIONS}

Machining is a manufacturing process, which removes unwanted material from a part, usually in the form of a chip. Virtually every product has components that require machining to a high degree of precision. The machining process is one of the most important manufacturing processes due to the value added to the finished product; however, it can also become the most expensive process as well.

\subsubsection{Types of Machining Processes}

All machining processes fall into one of the following major classes of cutting operations: lathe turning, planing, milling, drilling, and grinding [1,2]. In this section, the principles applicable to the process studied in this project, which is lathe turning, will be discussed. In turning, the workpiece is placed on a rotating apparatus (lathe) and a stationary single-point cutting tool is used to remove the unwanted material from a revolving workpiece, producing a new surface. A schematic of the turning process is shown in Figure 2.1. Although most common cutting operations are oblique 
(three-dimensional), the turning process can be reduced to an orthogonal (two-dimensional) analysis under the condition that the cutting edge is perpendicular to the cutting direction. The geometry resulting from making an orthogonal cutting approximation is shown in Figure 2.2. The symbols on this figure are explained in Section 2.1.2, and the deformation zones are described in Section 2.1.3.

\subsubsection{Machining Input Variables}

The outcome of a machining operation is highly dependent on the machining parameters, which fall into three categories: 1) workpiece properties, 2) cutting tool properties, and 3 ) cutting parameters.

\section{Workpiece Properties}

The properties of the workpiece include the workpiece geometry and material properties. The geometry of the workpiece to be machined is determined by the prior shaping process, such as casting or forging. The initial workpiece geometry and the desired workpiece geometry are factors, which establish the type of machining operation to be used. The metallurgy of the workpiece determines the ease with which the material can be machined. For example, more ductile materials such as copper machine easily, while titanium does not [3].

\section{Cutting Tool Properties}

The properties of the cutting tool include its geometry and material properties (or metallurgy). Tool geometry that can be adjusted includes rake angle, clearance angle, and tool tip radius (Figure 2.2). The rake angle $(\alpha)$ is defined as the angle between the rake face of a tool and a line perpendicular to the cutting direction. The clearance angle $(\gamma)$ is defined as the angle between the clearance face of the tool and the cutting direction. To ensure a reliable operation, the tool material properties must be superior to those of the workpiece being cut. Most frequently tool materials are high-speed steel (HSS), carbides, and coated tools. 


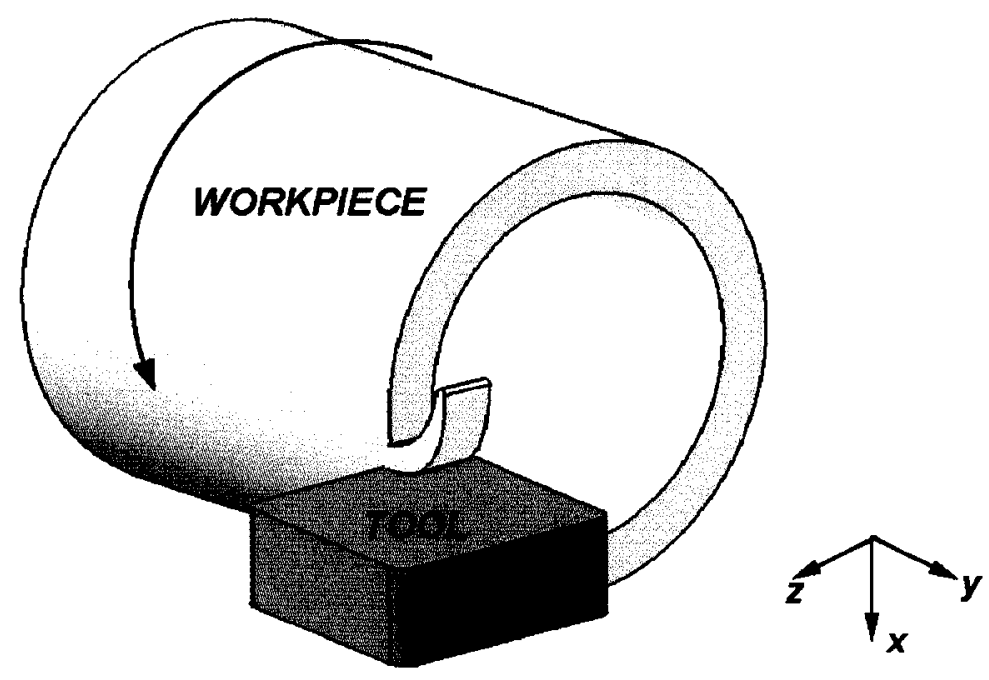

Figure 2.1: Schematic diagram showing the workpiece and tool geometry during the process of orthogonal metal cutting.

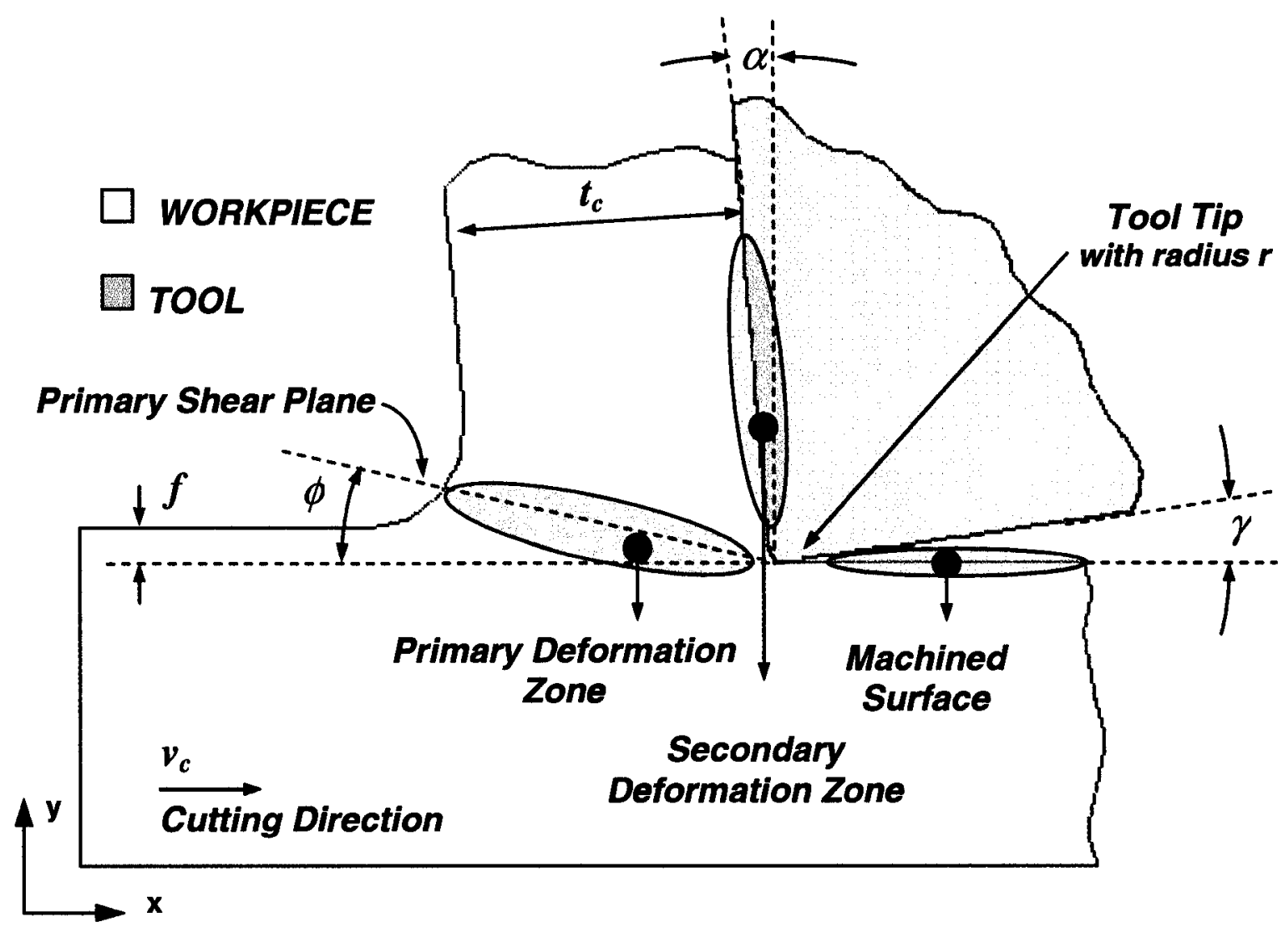

Figure 2.2: Schematic diagram of deformation zones and cutting parameters labelled on a cross-sectional workpiece undergoing orthogonal cutting, where $t_{c}$ is the chip thickness, $\alpha$ is the rake angle, $\gamma$ is the clearance angle, $f$ is the feed, $v_{c}$ is the cutting speed, and $\phi$ is the primary shear plane angle. 


\section{Cutting Parameters}

Cutting parameters include cutting speed, feed, and depth of cut; these parameters control the rate of material removal during the machining operation. The cutting speed $\left(v_{c}\right)$ is defined as the speed of the lathe rotation in a turning operation. The depth of cut $\left(t_{c u t}\right)$ is defined as a distance, perpendicular to the cutting direction, between the surface machined in the previous tool pass and the surface exposed in the current tool pass. The feed $(f)$ is the cutting depth per one cutting edge and one revolution. In the turning operation where a single edge cutting tool is used, the feed and the depth of cut are equivalent. Figure 2.2 illustrates the cutting velocity $\left(v_{c}\right)$ and feed/depth of cut $(f)$ in a turning operation.

In order to optimize the process of machining, the input variables must be chosen to fulfill functional requirements while providing a reliable service, or in other words prolonging tool life and/or achieving good surface finish. Tool life is dependent on the interaction of all input variables, and due to the infinite number of their combinations predicting the outcome of a machining process and its reliability are difficult.

\subsubsection{Deformation Mechanisms}

During the cutting process, the workpiece undergoes severe, and often highly localized, plastic deformation with strains in the order of 2 to $8 \mathrm{~mm} \cdot \mathrm{mm}^{-1}$ [4] and strain rates of $10^{3}$ to $10^{7} \mathrm{~s}^{-1}$ [5]. Three deformation zones are present during metal cutting and chip formation: primary deformation zone, secondary deformation zone and tertiary deformation zone (or machined surface), shown in Figure 2.2. As the tool edge advances and penetrates through the workpiece, the material directly ahead of the tool tip shears towards the free surface, or the chip root, and forms a localized plastic deformation zone referred to as the primary deformation zone (PDZ). Early assumptions were that the shear occurs along a single plane called the primary shear plane [7-13]. The angle between the cutting direction and the primary shear plane is called the shear angle, and is labelled as $\phi$ in Figure 2.2. The chip formed through this shearing process slides along the rake face of the tool, and deforms further in what is referred to as secondary deformation zone (SDZ). The deformation at the SDZ is caused by friction between the chip and tool rake face. The thickness of the chip, $t_{c}$ in Figure 2.2, is measured from the 
rake face to the free surface of the chip along a line perpendicular to the rake face. Finally, the newly machined surface deforms in the tertiary deformation zone due to friction between itself and the flank of the tool. The length of contact between the chip and tool is highly dependent on the input variables, as are the size and existence of the sticking and sliding regions within the contact length [6]. Due to the high chip-tool interface temperatures and pressures, the material adjacent to the tool tip seizes to the rake face, which is the region classified as the sticking region. In the sliding region the chip stops sticking to the tool and moves along the rake face with a constant sliding friction coefficient, until the contact length is reached.

\subsubsection{Theoretical Models of Metal Cutting Operations}

One of the earliest theories of material deformation during metal cutting, assumes a 'stack of cards' material displacement in the chip (Figure 2.3). Merchant [7] and Piispanen [8], assumed that the material in the chip displaces along a single shear plane forming a series of thin lamellas of material, which are so thin as to produce a uniform chip thickness. An expression for the orientation of this shear plane, or the shear angle $(\phi)$, was derived by Merchant [9] using the principle of minimum energy. Lee and Shaffer [10] and Shaw et al. [11], also derived an expression for $\phi$, but did so using the slip line field approach. Details of the models developed in reference [10] are given in Section 2.1.5.2. Rowe and Spick [12] estimated shear angles by minimizing cutting energy, an approach that was expanded upon by Wright [13], who also accounted for the energy consumed in the SDZ.

The concept of the deformation along a single shear plane was expanded by Recht [14] and Okushima and Hitomi [15]. Recht [14] extended the shear plane to a very thin rectangular zone where plastic deformation is concentrated. By machining a sample with markers of original material orientation, Okushima and Hitomi [15] were able to observe a fairly large deformation zone around the tool tip of an approximate triangular shape, and referred to it as the 'flow region'.

The significance of workpiece material properties, such as stacking fault energy and dislocation density, on machinability were studied by Black [16], Ramalingam and Black [17], and Lemaire and Backofen [18], through electron microscopy 


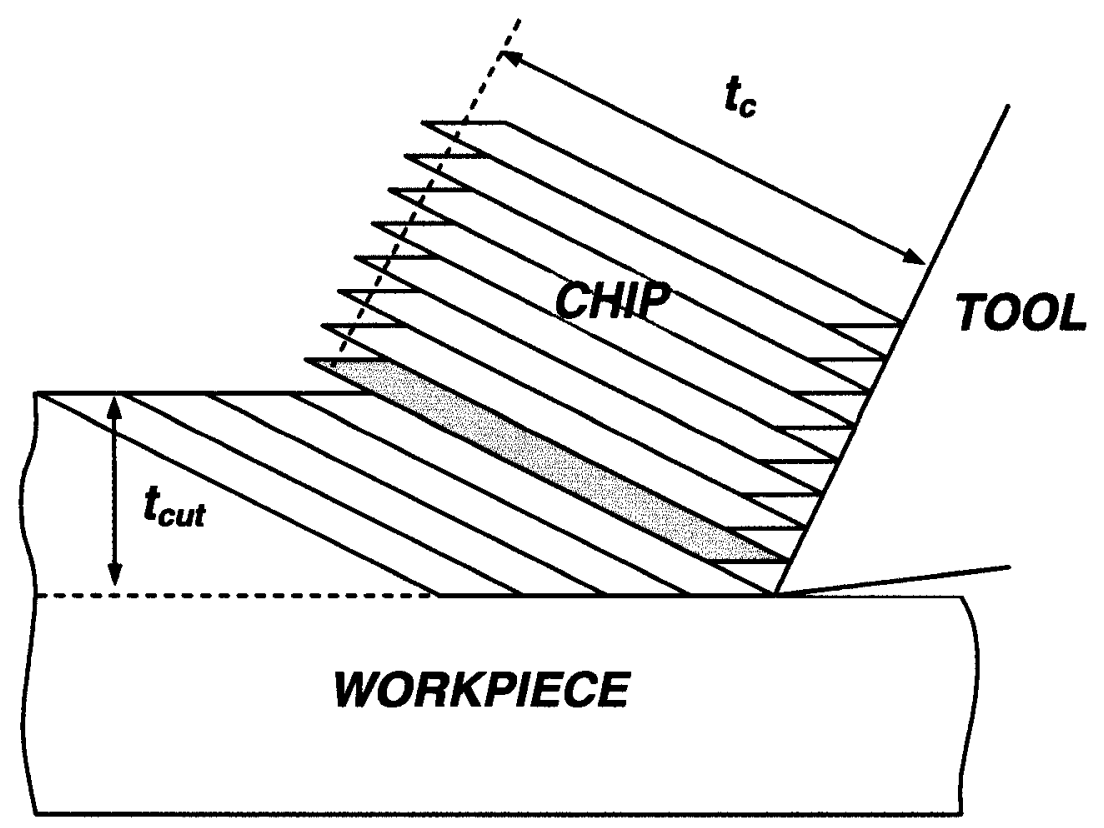

Figure 2.3: Idealized model of the cutting process developed by Piispanen [8], where the shaded lamellar has just moved.

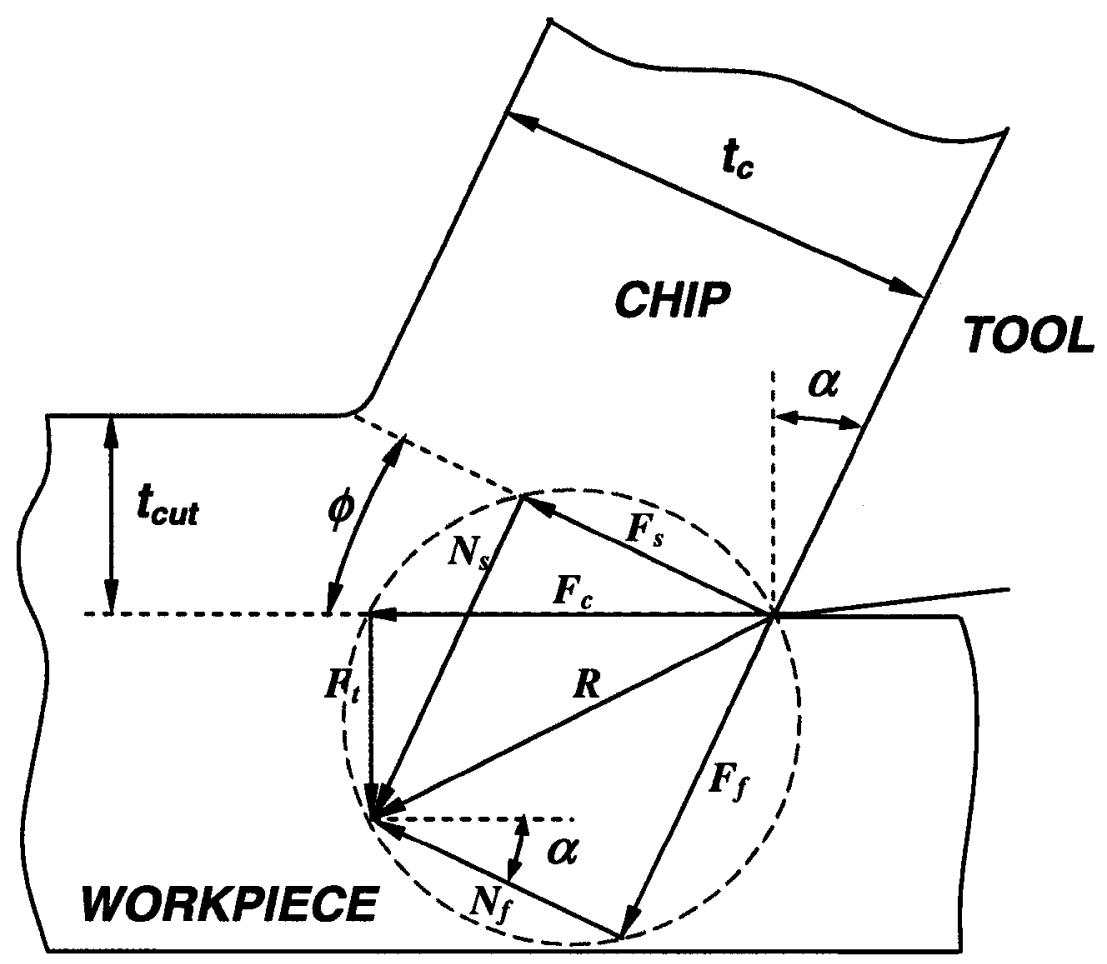

Figure 2.4: Force diagram developed by Ernst and Merchant [20], with the assumption of a single shear plane. 
investigations of the plastically deformed microstructure in machined samples. Further studies completed by Ramalingam and Black [19] concluded that chip formation is dominated by shearing within a thin shear front, which occurs when an equilibrium is established between strain hardening and dynamic recovery. Von Turkovich [4] obtained results in support of the equilibrium theory in [19] by proposing that the stress saturates above a certain strain, which accounts for the eventual equilibrium between work hardening and dynamic recovery.

\subsubsection{Mechanics of Chip Formation}

Based on the Piispanen model [8], Ernst and Merchant [20] generated a force diagram for orthogonal metal cutting (Figure 2.4), which has since been frequently used in the study of chip formation. Several assumptions were made by Piispanen in his model and are therefore applicable to the force analysis; they are:

i. Tool tip is perfectly sharp, therefore the workpiece does not contact the clearance face.

ii. Shearing occurs along a flat plane extending from the tool tip to the chip root, which is a sharp point formed by the chip free surface and previously machined surface.

iii. The width of cut is much greater than the feed, hence deformation occurs under plane strain, and radial forces are assumed to be zero.

iv. The chip is continuous and no built-up edge develops.

v. Shear stress, strain, and strain rates are uniform.

\section{Force, Stress and Power Consumption}

The following relationships are derived from the force equilibrium diagram in Figure 2.4:

$$
\begin{aligned}
& F_{s}=F_{c} \cos \phi-F_{t} \sin \phi \\
& N_{s}=F_{c} \sin \phi+F_{t} \cos \phi \\
& F_{f}=F_{c} \sin \alpha+F_{t} \cos \alpha \\
& N_{f}=F_{c} \cos \alpha-F_{t} \sin \alpha
\end{aligned}
$$


where $F_{c}$ is the cutting force, $F_{t}$ is the thrust force, $F_{s}$ and $N_{s}$ are the shear and normal forces on the shear plane, and $F_{f}$ and $N_{f}$ are the shear and normal forces on the rake face. The analysis of forces and stresses along the rake face is based on Coulomb sliding friction [21]; therefore the sliding coefficient of friction along the rake face is:

$$
\mu=\frac{F_{f}}{N_{f}}
$$

where $\mu$ is the coefficient of friction. The force diagram can further be used to determine the magnitude of the shear and tensile stress on the shear plane by diving the force by the area of the shear plane:

$$
\begin{aligned}
\tau_{s} & =\frac{F_{c} \cos \phi-F_{t} \sin \phi}{\left[\frac{w \cdot f}{\sin \phi}\right]} \\
\sigma_{s} & =\frac{F_{c} \sin \phi+F_{t} \cos \phi}{\left[\frac{w \cdot f}{\sin \phi}\right]}
\end{aligned}
$$

where $\tau_{s}$ is the shear stress on the shear plane, $\sigma_{s}$ is the normal stress on the shear plane, $w$ is the width of the chip (or tube wall thickness in turning). Such calculations cannot be performed for the rake face, since the stresses are not constant throughout the entire contact length due to the existence of the sticking and sliding regions (Section 2.1.3). The variation of normal stress $\left(\sigma_{f}\right)$ along the rake face is determined by a power law:

$$
\sigma_{f}=\sigma_{\max }\left(\frac{x_{c}}{l_{c}}\right)^{a}
$$

where $\sigma_{\max }$ is the maximum stress along the rake face and occurs at the tool tip, $x_{c}$ is the distance from the point at which the chip leaves the tool, $l_{c}$ is the length of contact between the tool and chip, and $a$ is a constant dependent on the material and tool properties. This relationship was developed on the basis of numerous experimental observations, which showed that normal stress increased along the rake face with increasing distance from the tool tip. The shear stress on the rake face is equal to the yield shear stress $\left(\tau_{y}\right)$ in the region where the chip seizes to the tool, or the sticking region. Since the friction in the sliding region obeys the Coulomb law of friction [21], the shear stress in the this region can be related to the normal stress by: 


$$
\tau_{f}=\mu \cdot \sigma_{\max }\left(\frac{x_{c}}{l_{c}}\right)^{a}
$$

The total power, or energy, required to complete the machining process is the combination of energy required to displace the material along the shear plane, and the energy required to displace the material along the rake face of the tool. The equation for the total power $\left(P_{t}\right)$ required to complete the process according to reference [3] is expressed as:

$$
P_{t}=F_{c} v_{c} .
$$

Equation (2.10) establishes that the power consumption in machining is directly related to the cutting force; therefore the process should be geared to minimize this force. By examining the force equilibrium in Figure 2.4, the cutting force decreases with an increasing shear angle $(\phi)$; as such studies on the value of this shear angle are important in controlling power consumption.

\section{Shear Plane Angle}

Ernst and Merchant [20], used the minimum energy principle to develop the following expression for the shear plane angle $(\phi)$ :

$$
\phi=\frac{\pi}{4}+\frac{\alpha}{2}-\frac{\beta}{2}
$$

where $\beta$ is an angle associated with the resultant of the shear and normal forces on the rake face $\left(F_{f}\right.$ and $\left.N_{f}\right)$ and can be expressed as:

$$
\beta=\tan ^{-1}\left(\frac{F_{f}}{N_{f}}\right)=\tan ^{-1}(\mu) .
$$

Lee and Shaffer [10] used the slip line field model to approximate the shear angle and developed the following expression:

$$
\phi=\frac{\pi}{4}+\alpha-\beta
$$

In practice, equations developed by both Ernst and Merchant [20], and Lee and Shaffer [10], do not yield accurate shear angle predictions owing to the oversimplified assumptions that shearing occurs along a single plane extending from the tool tip to the chip root. However, these equation can sufficiently enough provide insight into the 
manipulation of some variables, which can result in maximization of the shear angle $(\phi)$ and thus minimization of the process power consumption, as mentioned in the previous section. From shear angle approximations in both references [10] and [20], $\phi$ is maximized by increasing the rake angle $(\alpha)$ and decreasing the angle $\beta$. Increasing the rake angle is simply a matter taken into account during tool design, whereas decreasing the angle $\beta$ can be achieved by decreasing the friction coefficient on the rake face as indicated by Equation (2.12).

Therefore, in order to decrease power consumption of a machining process, the rake angle of the tool should be increased, as much as possible, and the friction coefficient on the chip-tool interface should be minimized by either applying lubricants or using materials with low coefficients of friction.

\subsection{FINITE ELEMENT MODELING}

FE analysis has become a vital part of mechanical design. As an alternative to prototype testing, it is a comparatively inexpensive and swift method of assessing concept designs and design improvements. The FE method subdivides a continuous body into finite size elements interconnected by nodal points on the element edges. Time integration techniques predict a real-life continuous phenomenon by approximating the displacement of each of the nodes in the discrete body at finite time intervals. Two types of mechanical analysis can be performed through FE modeling: quasi-static and dynamic. A quasi-static problem is classified as one occurring over a long period of time, without significant load or boundary condition variations. A dynamic analysis occurs over a short time interval, within which the loads and boundary conditions are highly time dependent. A machining operation, such as the one studied herein, is of a dynamic nature; hence focus in this section is placed on a dynamic analysis [22].

Non-linear FE analysis consists of five main steps:

1) Model development

2) Formulation of governing equations

3) Discretization of equations 
4) Solution of equations

5) Interpretation of results

Commercially available FE codes, such as LS-DYNA, which was used in the present work, encompass steps 2 through 4 . These steps are summarized in section 2.2.1. The subsequent sections $(2.2 .2-2.2 .7)$ summarize several topics important to model development and interpretation of results, as applicable to the present research.

\subsubsection{LS-DYNA Fundamentals}

LS-DYNA is an explicit FE program, developed by Livermore Software Technology Corporation (LSTC), and capable of performing non-linear dynamic analyses of three-dimensional structures [23].

The governing equations used in an analysis are common to most FE codes. In addition to the equation of state and constitutive relation, the dynamics of a continuous media are established through a series of differential equations derived from the principles of conservation of mass, momentum and energy. As the continuous media is subdivided into discrete elements, the governing equations must also undergo discretization. The principle of virtual work is used in defining the mechanical equilibrium and establishing the discrete equations.

$$
\delta W=\delta W^{I n t}-\delta W^{E x t}+\delta W^{K i n}=0
$$

In Equation (2-14), $\delta W$ is the total virtual work, $\delta W^{I n t}$ is the virtual work done by internal forces, $\delta W^{E x t}$ is the virtual work done by external forces acting on the body or the surface, and $\delta W^{K i n}$ is the kinetic virtual work resulting from inertial forces. For the principle of virtual work to hold, the internal virtual work (a result of the virtual strain and true stress fields), must be balanced by the external components of virtual work (those resulting from external forces). The expressions for each of the components of virtual work are based on the governing equations, which establish time dependence within the equation. By employing corresponding boundary conditions and shape functions to account for special dependence of displacement, a matrix equilibrium equation can be established, also referred to as the governing equation of motion with the 
general form:

$$
[M] \cdot\{\ddot{u}\}+[C] \cdot\{\dot{u}\}+[K] \cdot\{u\}=\left\{F_{\text {ext }}\right\}
$$

where $[M]$ is the mass matrix, $\ddot{u}$ is a nodal acceleration vector, $[C]$ is the damping matrix, $\dot{u}$ is a nodal velocity vector, $[K]$ is the stiffness matrix, $u$ is the nodal displacement vector, and $\left\{F_{e x t}\right\}$ is the externally applied force. Appendix A contains an example of discretization procedures for a 2-dimensional axially loaded member, as outlined by Zamani and Altenhof [24].

There are two distinct types of methods for the solution of the equation of motion available in FE analysis; they are the explicit and implicit time integration methods. The time integration algorithm used for the explicit code is the central difference scheme [25], while the implicit code uses the Newmark's method [26], both of which are explained in Appendix B. The main advantages of the explicit scheme are that the $[K]$ matrix does not require inversion and that $\dot{u}$ and $\ddot{u}$ are linear functions of time during a time interval, which can prove beneficial for highly non-linear problems. The limitation of the explicit scheme lies in its conditional stability where there exists a time step restriction, which can significantly prolong simulation time. While the implicit scheme is unconditionally stable with respect to the size of the time step, it requires the inversion of the stiffness matrix $[K]$, which can be difficult depending on the complexity of the problem at hand and may result in prolonged computation times.

Due to the highly dynamic and non-linear nature of the machining process, the explicit scheme was used for the simulations performed in the scope of this project. The explicit time integration proceeds according to the flow chart presented in Figure 2.5, obtained from Belytschko et al. [23]. The flowchart reflects a governing equation of motion:

$$
[M]\{\ddot{u}\}=\left\{F^{e x t}\right\}-\left\{F^{\text {int }}\right\}
$$

where $\left\{F^{e x t}\right\}$ are the external forces on the element and $\left\{F^{i n i}\right\}$ are the internal forces on the element resulting from stress and strain within the element. The flowchart shows that 


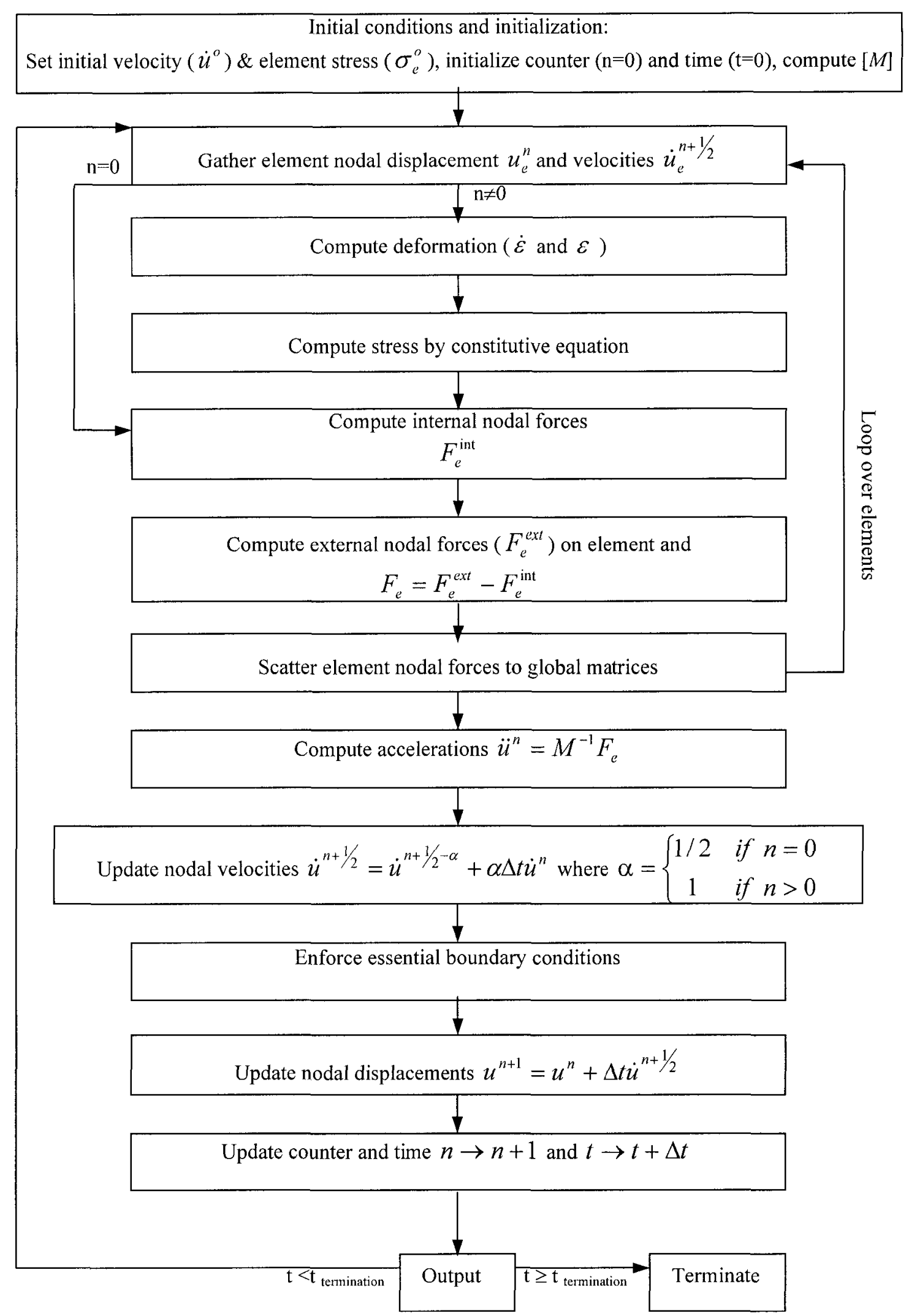

Figure 2.5: Flowchart for explicit time integration of a Lagrangian (Section 2.2.2) mesh, obtained from Belytschko et al. [23]. 
the mass matrix $[M]$, and forces $\left\{F^{e x t}\right\}$ and $\left\{F^{i n t}\right\}$ within the element, are computed first, which allows for computation of acceleration $\{\ddot{u}\}$ through equation (2-16).

\subsubsection{Eulerian, Lagrangian and ALE Element Formulations}

Three element types are available in LS-DYNA, they include: Lagrangian, Eulerian, and Arbitrary Lagrangian-Eulerian (ALE) elements. Coordinates of the distinctive meshes are subdivided into two categories: 1) spatial coordinates, denoted as $X_{s}$, and 2) material coordinates, denoted by $X_{m}$. Spatial coordinates are points defined and fixed to space, and therefore do not change throughout the duration of the simulation. Material points, on the contrary, are defined with reference to a material coordinate and remain fixed to the material coordinates throughout the simulation.

The simplest way to differentiate between an Eulerian and Lagrangian mesh, is to compare the behaviour of the mesh and its nodes. The initial configuration of a mesh in a FE simulation is such that the material points are aligned with spatial points or $X_{s}=X_{m}$. When a Lagrangian mesh undergoes deformation, the nodes of the mesh remain fixed to material coordinates, therefore the Lagrangian mesh moves with the material. During every time step the Eulerian formulation also undergoes a Lagrangian step; however at the end of every time step the mesh is remapped to its original spatial coordinates, a process referred to as an advection step. Since, the Eulerian mesh is constantly brought back to its original position, during post-processing it appears to be fixed to the spatial coordinates. While the mesh is remapped to its original position, the material is not; therefore an airmesh must surround the original mesh to enclose the deformed material. The airmesh is referred to as such, since it originally contains no material (much like air) and its only purpose is to provide ample space for material deformation outside of the original material mesh. Care must be taken in modeling the airmesh large enough to account for any possible material deformation during the simulation. The dynamics of the Lagrangian and Eulerian mesh are best described by the illustration in Figure 2.6. When the original material contained in a Lagrangian mesh ABCD in Figure 2.6 (a) deforms, the mesh deforms coincidentally with the material coordinates. The same material contained in an Eulerian mesh ABCD in Figure 2.6(b), deforms independently of the 

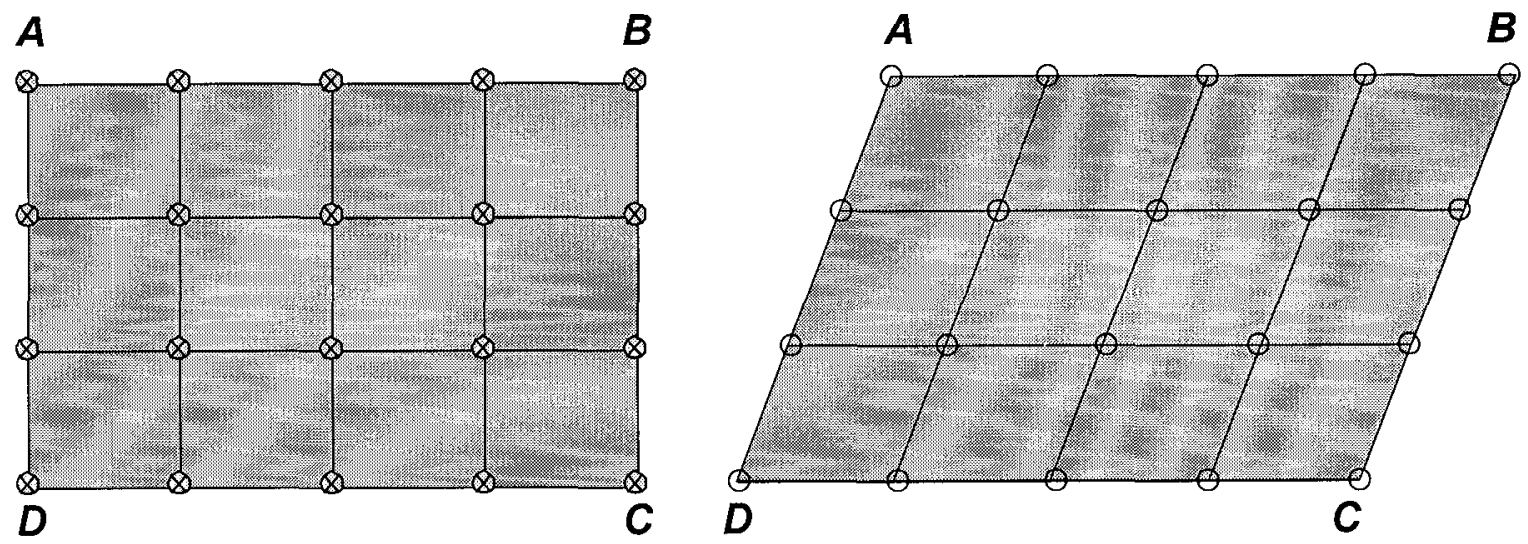

(a) Lagrangian mesh behaviour

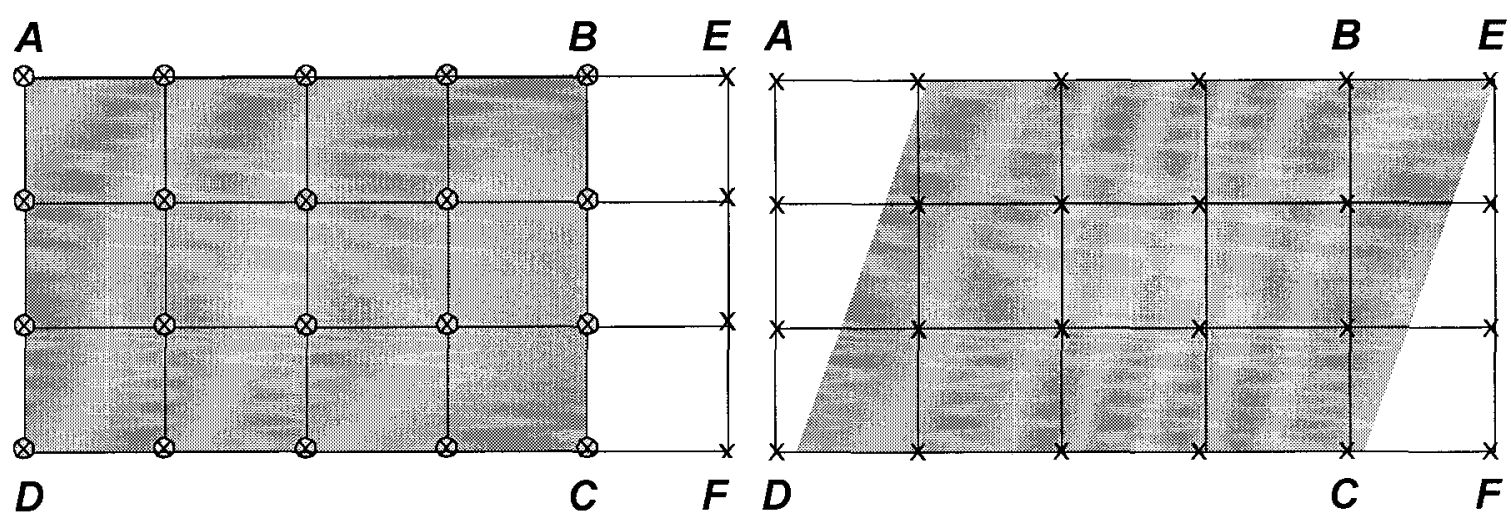

(b) Eulerian mesh behaviour

\section{Material X Spatial Coordinates $\bigcirc$ Material Coordinates}

Figure 2.6: Two-dimensional mesh illustrating the behaviour of: (a) Lagrangian mesh, and (b) Eulerian mesh, with respect to material deformation. 
mesh and therefore an additional "airmesh" BEFC is needed to provide mesh space to accommodate material deformation.

The last remaining element formulation available in LS-DYNA software is the ALE formulation, which is a hybrid of the Eulerian formulation. ALE and purely Eulerian formulations parallel in their Lagrangian step but differ in the advection step. While the Eulerian mesh is always remapped back to its original position during the advections step, the ALE mesh is remapped to an entirely new mesh smoothed only to a level that eliminates excessive distortion. The smoothing can be performed by the following smoothing weight factors: 1) simple average, 2) volume weighting, 3) equipotential, and 4) equilibrium. Each of the smoothing algorithms has a unique approach to determining the coordinates of nodes in the advection step, and all algorithms are detailed in the LS-DYNA Theoretical Manual [27].

In both the Eulerian and ALE formulation, the advection method can be: 1) first-order donor cell where properties are assumed constant throughout the element, or 2) second-order Van Leer to allow the interpolation of properties within an element into a piecewise function by methods described in $[28,29]$.

\subsubsection{Contact Algorithms}

The modeling of contact or impact in FE simulations is one of its most important aspects but often proves to be very difficult. Contact occurs when two or more bodies come together and their interaction must be computed. The most important condition for contact is impenetrability, which means that one body cannot penetrate into another. In order to uphold impenetrability and compute forces necessary to do so, the penalty stiffness method [27] is used. This most frequently used contact search algorithm in LS-DYNA decomposes bodies in contact into two parts, the slave and the master, and assumes that slave nodes cannot penetrate through master surfaces. Imaginary interface springs are placed between penetrating nodes and contact surfaces, with a (penalty) stiffness based on geometric and material properties of the contacting entities. This stiffness is defined as:

$$
k=C_{\text {user }} \cdot K \cdot \frac{A_{c}{ }^{2}}{V}
$$


where $C_{u s e r}$ is the user defined scale factor for the penalty stiffness (of imaginary springs), $K$ is the bulk modulus equal to $\frac{E}{3(1-2 v)}, A_{c}$ is the face area of the element in contact and $V$ is the volume of the element.

Many contact algorithms exist in LS-DYNA to define contact between two Lagrangian entities. The contact between the master and slave can be "automatic" or "manual", where the "manual" method requires the user to create segment sets on contacting faces of entities while the "automatic" method prompts LS-DYNA to create segment sets on user defined parts and part sets. The automatic input of contacting segment is advantageous in that it covers majority of contact and is much less time consuming for the user since it decreases modeling difficulty; however, the simulation time is longer and user has no control over where contact occurs. Choosing contact segments manually is very time consuming and carries with it the possibility of incomplete contacting area definition; however, it does decrease CPU time since only the specified segments are checked for penetration. When using the automatic contact option, it is important to orient segment normals towards the contacting entity since interface forces can only act in the direction of the normal. If normals are not defined, the interface forces can act to push nodes to penetrate the master surface rather then preventing penetration.

Friction in the contact algorithm of LS-DYNA is based on the Coulomb formulation [21], such that:

$$
\tau=\mu_{c} \cdot \sigma_{n}
$$

where $\tau$ is the shear stress on the interface, $\mu_{c}$ is the coefficient of friction calculated by LS-DYNA and $\sigma_{n}$ is the stress normal to the contacting interface. The LS-DYNA contact algorithms are capable of modeling a varying coefficient of friction $(\mu)$. The friction coefficient varies between the static and dynamic value, as a function of relative velocity of the surface in contact, shown in the following equation:

$$
\mu=\mu_{d}+\left(\mu_{s}-\mu_{d}\right) \cdot e^{-D C\left|v_{r e l}\right|}
$$

where $\mu$ is the calculated coefficient of friction, $\mu_{d}$ is the dynamic coefficient of friction, $\mu_{s}$ is the static coefficient of friction, $D C$ is the user defined decay coefficient, and $v_{r e l}$ is 
the relative velocity at the contact surface. This type of friction definition enables the user to define the gradient of the transition from static to dynamic coefficient of friction by altering the decay coefficient $D C$.

Contact is not limited to nodes and surfaces; a contact can also be defined between two surfaces or between a surface contacting itself. Contact cards also exist to model spot welds, airbag deployment and eroding surfaces, as well as constrained surfaces and tied surfaces, just to list a few. The capabilities of modeling contact between two Lagrangian entities in LS-DYNA are listed under the ${ }^{\star}$ CONTACT_OPTION in reference [30].

Modeling contact between a Lagrangian and an Eulerian mesh slightly differs from that described for two Lagrangian entities. Primarily it is not through *CONTACT_OPTION cards but rather by the *CONSTRAINED_LAGRANGE_IN_SOLID card, which couples Lagrangian elements to the nodal points of an Eulerian mesh. In this type of contact the Lagrangian mesh is declared the slave and the Eulerian mesh is the master. The penalty stiffness method previously described is also applicable in this contact algorithm; however, in addition to monitoring penetration at nodes this algorithm can also monitor penetration by a virtual rectangular grid of $n \times n$ points on the surface of each element. Other methods of preventing penetration include constraining the acceleration and/or velocity of the Eulerian material at the Lagrangian surface. The main deficiency of the Eulerian-Lagrangian contact is the friction definition, which is limited to a constant value, unlike the variable friction function available in ${ }^{\star}$ CONTACT_OPTION algorithms.

\subsubsection{Material Properties}

All FE simulations require some type of material property definition to determine the stresses, strains, displacements, velocities, and accelerations. The quality of the developed material model is very important in generating accurate simulation results. A good material model should address all aspects of material behaviour including, but not limited to: elastic deformation, plastic deformation, and effects of strain rate and temperature. An elastic material follows a constant slope characterized by the Young's modulus $(E)$ and does not undergo any plastic deformation. A purely plastic material 
contains an elastic region with slope $E$ until a yield strength is reached $\left(\sigma_{y}\right)$ and thereafter the stress remains at a constant value of $\sigma_{y}$ for any applied strain. An elastoplastic material, undergoes work hardening.

LS-DYNA defines material behaviour through three methods, they include: 1) binary approximation, 2) constitutive equations, and 3) direct input of stress-effective strain data points. Typically material data is obtained from experimental testing such as a uniaxial tension test for low strain rates, torsion test for high strains but low strain rates, and Taylor bar tests for high strains and high strain rates. These data require some manipulation since the stress and strain entered in LS-DYNA should always be true unless otherwise specified. Over 100 material models are available in the LS-DYNA code, including models designed to replicate the behaviour of composites, ceramics, polymers, metals, and fluids, including specialized types of materials such as: foams, glass, fabric, hydrodynamic materials, plastics, rubber, soil, wood, and heart and lung tissue. When choosing from these material models, some important aspects in need of consideration are the levels of strain rate and temperature dependence. For example, strain rate effects should always be taken into account when modeling highly rate sensitive materials, such as steel. Another characteristic, which requires consideration is the objective of the simulation: whether it is important to monitor plastic deformation alone, or is it also essential to model fracture or crack initiation and growth.

Under certain circumstances, a material model ( ${ }^{\star M A T}$ OPTION) relating stress to strain is insufficient for analysis. For example, when a thermal analysis is being conducted, the material definition also requires a thermal material model ( ${ }^{*}$ MAT_THERMAL_OPTION). When a material model is used in modeling hydro materials or

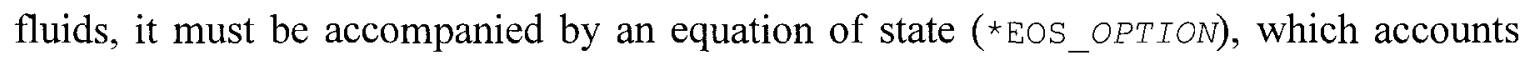
for resistance to hydrostatic compression [31].

Each of the material models has its distinct characteristics, and not all models are compatible with each element formulation and element type. The details of the material models and their applicability to the analysis being conducted, are contained in LS-DYNA Keyword User's Manual Volume II [32] and Theoretical Manual [27]. 


\subsubsection{Adaptivity in Lagrangian Element Formulation}

LS-DYNA incorporates in its code an h-adaptive method for shell elements. In this method, the shell elements are subdivided into smaller elements when original elements of the mesh lose accuracy due to mesh distortion, thereby controlling accuracy of the simulation with minimal increase in computational cost. The user sets the criteria for elements requiring refinement, in the form of a critical total angle change within an element or a critical angle change of an element relative to surrounding elements. A node is created at the midpoint of every edge in elements being adapted, and the original element (parent element) is split into 4 equally sized elements (descendant elements). The node coordinates, midpoint coordinates, nodal velocities, and midpoint angular velocities are all determined by linear interpolation, while parent element properties such as stress are directly applied to each descendant element. The user can specify the levels of refinement, or the number of times the descendant elements can be refined; where refinement levels of $1,2,3 \ldots$, correspond to the creation of 4,16 and $64 \ldots$, descendant elements from the parent element. The method used for two-dimensional solid elements (such as plane strain elements) is r-adaptivity, in which an entirely new mesh is generated from the original mesh by a least squares fit. The user defines a time at which the refinement is to occur and the characteristic element size for the refined mesh.

After a mesh refinement occurs, LS-DYNA can either continue calculations or back-up in time and repeat calculations with the refined mesh in case error arose prior to refinement. The first method is called one-pass adaptivity, illustrated in Figure 2.7 (b), and it is more efficient in computational time. The latter method is called two-pass adaptivity, illustrated in Figure 2.7 (a), and although it is more stable and avoids propagation of error, it is costly in computation time. H-adaptivity and r-adaptivity are discussed in more detail in reference [27].

\subsubsection{Structure of a Finite Element Input File}

The finite element input file, consists of a series of control "cards" used to dictate all aspects of the simulation; hence this file is often referred to as the input deck. It must

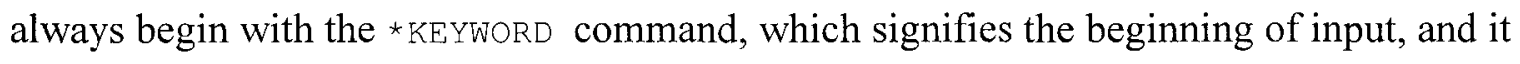




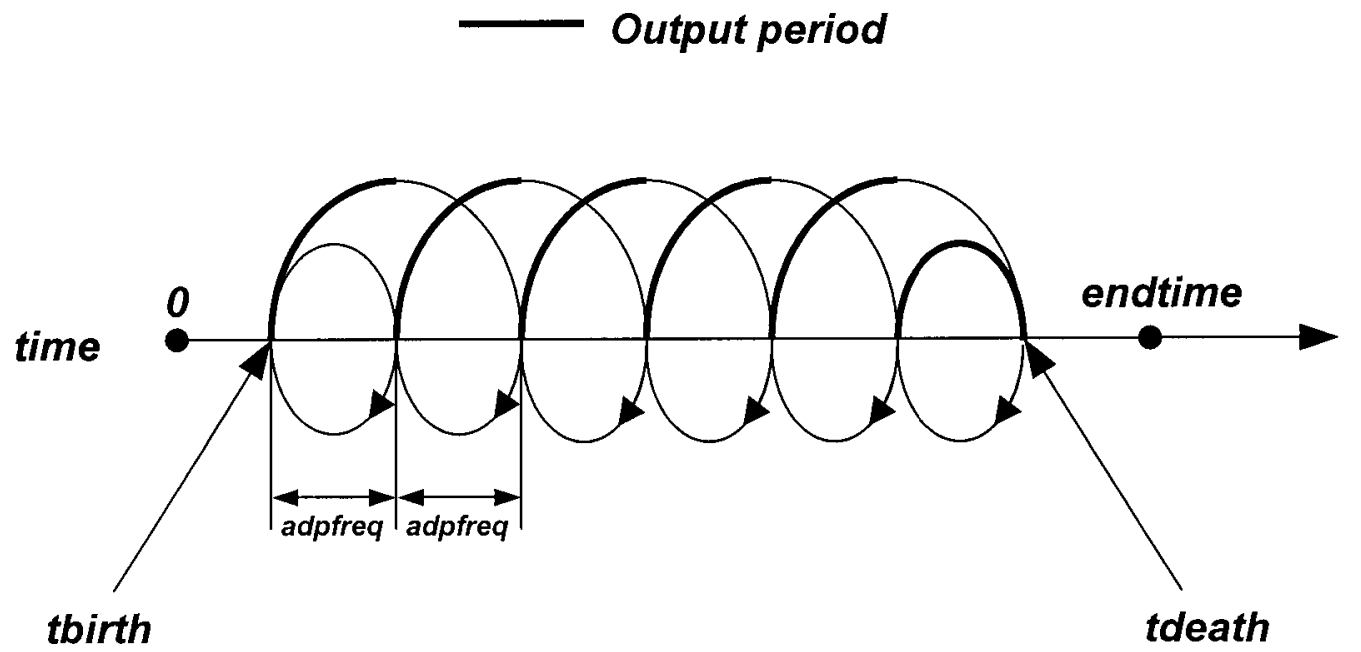

(a)

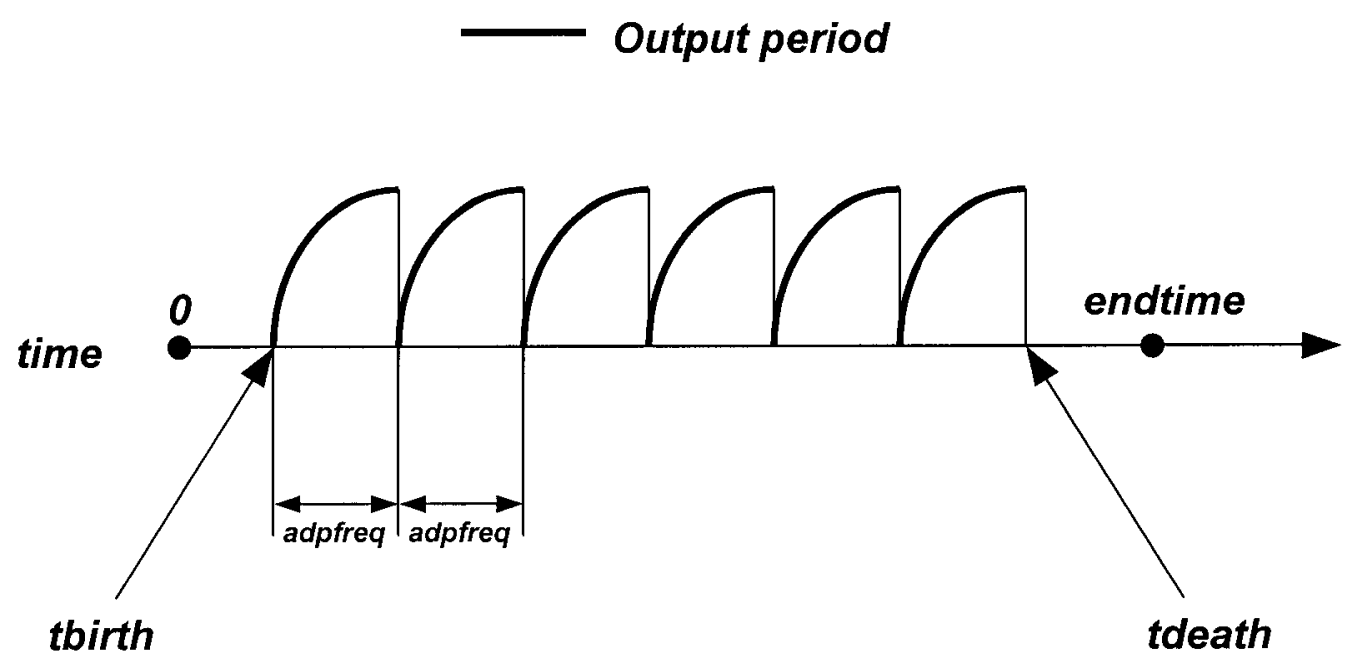

(b)

Figure 2.7: Adaptivity methods in LS-DYNA: (a) two-pass adaptivity where the calculation is repeated after adaptive remeshing, and (b) one-pass adaptivity where calculation is not repeated after adaptive remeshing. 
must always end with the ${ }^{*} E N D$ command, which indicates that the reading of input has been completed. The computation procedures are controlled through the ${ }^{\star}$ CONTROL_OPTION cards; although the definition of these cards is optional, it does help in optimizing the simulation by redefining parameters otherwise set to LS-DYNA defaults such as: specifying a termination time, defining the time step scale factor, activating the ALE element formulation or Lagrangian adaptivity, monitoring and controlling hourglass energy, etc. ${ }$ AATBASE OPTION cards control the output generated through the simulation, including all types of information collected and the time interval at which it is collected. Output files include binary files and ASCII files. The root of each model lies in the *PART cards, which contains the identification number for the part (PID) and reference to all material information required to complete the model such as: 1) the material properties identification number (MID), which links to the designated *MAT_OPTION card; 2) the section properties identification number (SECID), which links to the designated ${ }^{*}$ SECTION_OPTION card; and 3) material behaviour defined by an equation of state (EOSID), if applicable, which links to a designated ${ }^{*}$ EOS_OPTION card. The geometry of the model is defined in the ${ }^{*}$ NODE cards which define the $\mathrm{x}, \mathrm{y}$ and $\mathrm{Z}$-coordinates $(\mathrm{X}, \mathrm{Y}, \mathrm{Z}$ ), as well as the translational or rotational constraints ( $\mathrm{TC}$ and $\mathrm{RC}$ ) of all nodes in the entire model. The element definition, including its identification number (EID), nodes they are composed of (N1, N2, N3...), and parts to which they belong (PID), is in the $\star_{E L E M E N T}$ OPTION card. Additional cards, include those defining contact between two entities ( ${ }^{\circ}$ CONTACT_OPTION), those defining initial boundary conditions (*BOUNDARY_OPTION), and initial loads applied to entities (*LOAD_OPTION), etc; are also available for use in the LS-DYNA input deck. Every card that is available in LS-DYNA is fully detailed and explained in Volumes I and II of the LS-DYNA 970 Keyword User's Manual [30, 32].

\subsubsection{Interpretation of Results}

A method, by which the validity of a simulation is confirmed, lies in the energy balance of the simulation. Because energy can neither be created nor destroyed, the total energy (minus that energy accumulated through external work) should always equal to the initial energy; and hence the energy ratio, defined as the ratio between total and initial 
energy, should be 1.0. The types of energies incorporated in the energy balance of a system are: internal energy, kinetic energy, interface energy, hourglass energy, external work, etc. Energies such as hourglass energy and interface energy should be monitored. When elements with one integration point are used in a simulation, the displacement of the nodes can result in zero resultant displacement at the integration point as shown in Figure 2.8. This type of element deformation is referred to as the hourglassing or zero energy mode of deformation [23, 27]. The viscous and stiffness methods are available for controlling hourglassing effects. These methods apply additional forces related in magnitude to nodal velocities and displacements, respectively, to compensate for the hourglass deformation. In the energy balance, hourglass energy is work developed by the hourglass forces and nodal displacements to resist zero energy modes; this energy should be kept under $10 \%$.

Sliding interface energy is a result of the contact between the master and slave entities. The energy is calculated by:

$$
E_{\text {contact }}^{n+1}=E_{\text {contact }}^{n}+\left[\sum_{i=1}^{n s n} \Delta F_{i}^{\text {slave }} \times \Delta d_{i}^{\text {slave }}+\sum_{i=1}^{n m n} \Delta F_{i}^{\text {masser }} \times \Delta d_{i}^{\text {master }}\right]^{n+1 / 2}
$$

where $n s n$ and $n m n$ are the number of slave and master nodes, respectively. The interface forces between the $i$ th slave/master node and the contact segment are presented by $\Delta F_{i}^{\text {slave }}$ and $\Delta F_{i}^{\text {master }}$, respectively. The incremental distances that the $i$ th slave / master node have moved during the last time step are noted as $\Delta d_{i}^{\text {slave }}$ and $\Delta d_{i}^{\text {master }}$, respectively. When friction is not considered, the magnitude of the master energy and slave energy should be approximately equal in magnitude but opposite in sign. Friction will cause an increase in interface forces and will result in positive sliding interface or contact energy, which is acceptable. Negative sliding interface energy indicates penetration, and should be avoided. 


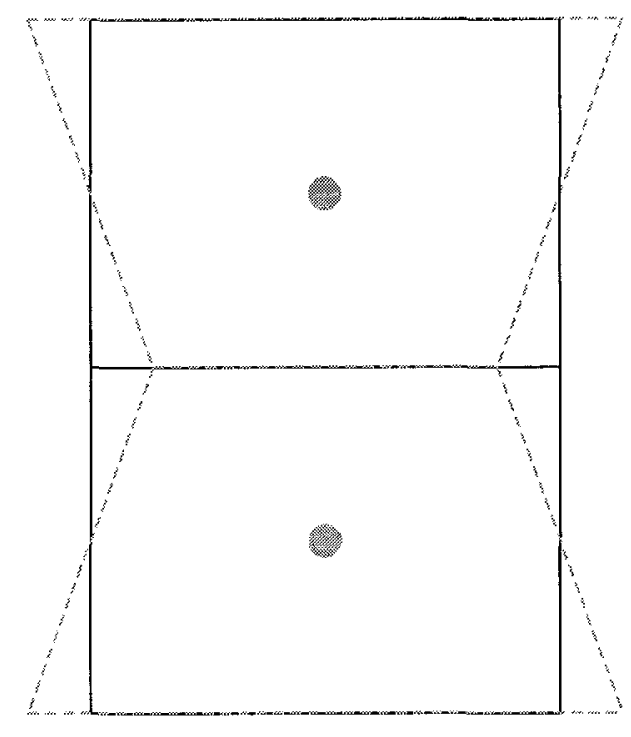

(a)
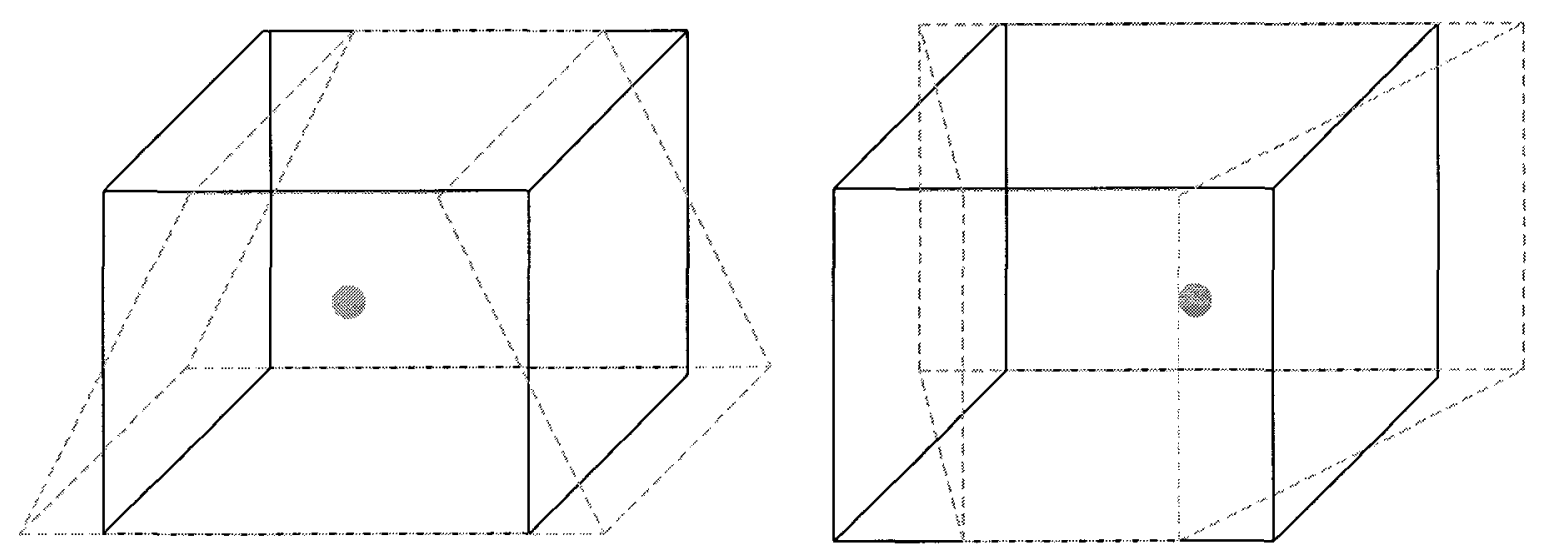

(b)

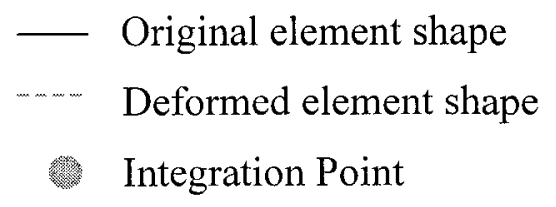

Figure 2.8: (a) Hourglass modes in shell elements (note the hourglass shape of deformed elements), and (b) two of twelve possible hourglass modes in solid elements. 


\subsection{EXISTING FINITE ELEMENT MODELS OF METAL CUTTING}

\subsubsection{Lagrangian Element Formulation}

One of the earliest FE models for metal cutting was developed by Klamecki [33], who performed simulations on a Lagrangian mesh studying the incipient chip formation, and performed experimental correlation on the shape of the chip cross-section at the initial stage. The model did not thrive largely due to significant modeling shortcuts, such as modeling the workpiece as a perfectly plastic material and disregarding friction. In the decade to follow, important advances were made in all areas of the model, including: material modeling, modeling of contact at chip-tool interface, and experimental correlation providing model verification. The material modeling of the workpiece, advanced from elastic-perfectly plastic behaviour [33, 34], to elastic-visco plastic behaviour [35, 36], and further to models accounting for strain, strain rate, and temperature effects [37]. The chip-tool interaction was accounted for using several methods, including defining a constant coefficient of friction at the interface [38], defining a distinct coefficient of friction for the two regions of contact (sticking and sliding) [39], or varying the frictional stress as a function of the normal stress [40]. The experimental correlations were limited if at all completed, and they included comparison of interfacial stress and/or temperature [35, 37], forces [35, 39, 40] or residual stresses and strains [34].

The most challenging aspect of modeling machining with a Lagrangian mesh is the inability of the mesh to support high strains such as those experienced in the chip formation. Due to the high strains in order of 2 to $8 \mathrm{~mm} \cdot \mathrm{mm}^{-1}$ [4] that accumulate in the workpiece material, and strain rates of $10^{3}$ to $10^{7} \mathrm{~s}^{-1}[5]$, the Lagrangian FE mesh can become severely distorted causing calculation inaccuracies. Techniques most frequently used by researchers to overcome the limitations of the Lagrangian mesh are to apply a failure criterion along a pre-determined line lying parallel to the cutting direction at the tool tip level, or to continuously adapt the mesh.

The separation criteria, which are applied along the cutting line, give a condition upon which nodes along this pre-determined line split or separate. Several types of conditions have been applied in past research, each based on physical and/or geometrical 
constraints. Physical conditions are those defined by critical stresses or strains, while geometrical are based strictly on the distance relative to the tool tip. Komvopoulos and Erpenbeck [41] applied a geometrical criterion to study the effect that friction coefficient and two plasticity workpiece models had on chip formation. In this case, superimposed nodes separated upon coming within a critical distance of $35 \mu \mathrm{m}$ to the tool tip, equal to one half of the element dimension ahead of the tool tip. The model assumed plane strain conditions and that the process was of quasi-static nature. The material models studied included: elastic-perfectly plastic (EPP), and elastic-plastic with isotropic strain hardening and strain rate sensitivity defined by a constitutive law (EPHSR). Each of the materials was modeled with a coefficient of friction of $0,0.15$ and 0.5 . The model was validated through good experimental-numerical cutting force and chip geometry correlation, with numerical results predicting cutting forces to within $6 \%$ and overestimating the chip thickness by $16 \%$. The distributions of stress developed in both the EPP and EPHSR materials are shown in Figure 2.9 (a) and (b), respectively. The distributions showed that accounting for work hardening and strain rate effect, considerably increased the stress value. For example, the maximum stress along the rake face increased from $480 \mathrm{MPa}$ to $800 \mathrm{MPa}$ when strain rate and hardening effects were considered. Similar increases in stress as well as strain were observed when the friction was increased, as illustrated by Figures 2.10 (a) and 2.10 (b). The strain along the rake face reached values of 0.6 when $\mu=0$, and increased to 0.8 for $\mu=0.15$. Although information in the research of Komvopoulos and Erpenbeck [41] reinforced some already understood effects of material modeling and friction on stress and strain distributions, it had limited experimental validation.

A geometrical criterion was also applied by Lin and Lo [42], with validation by numerical-experimental comparison in terms of cutting forces. Plane strain conditions were assumed for the model, and friction was assumed as constant along the chip-tool interface. By applying the Johnson-Cook constitutive equation, these researchers were able to incorporate temperature effects in addition to work hardening and strain rate effects. Conclusions drawn from the investigation were that increasing the temperature significantly reduced the numerical stresses within the chip and machined surface. When temperature effects were neglected, the stress in the chip varied from 200 to $500 \mathrm{MPa}$, 


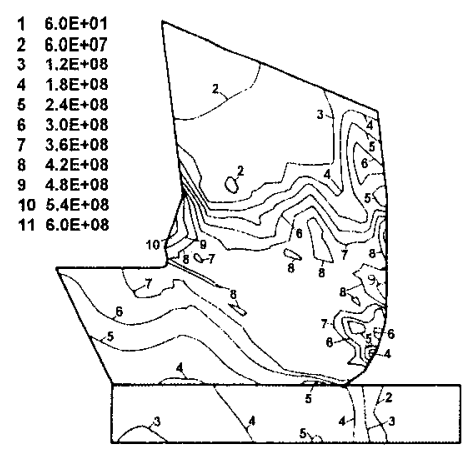

(a)

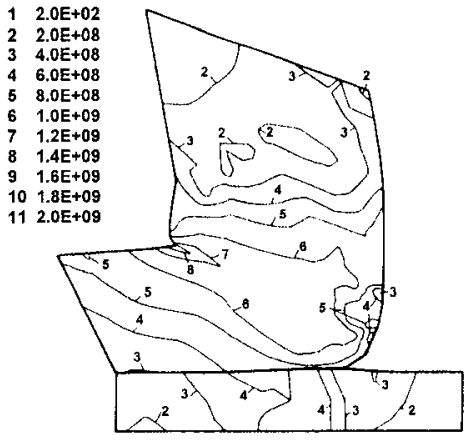

(b)

Figure 2.9: Distributions of stress, in units $\mathrm{Pa}$, for (a) elastic-perfectly plastic material, and (b) elastic-plastic material with hardening and strain rate effects; obtained from the work of Komvopoulos and Erpenbeck [41].

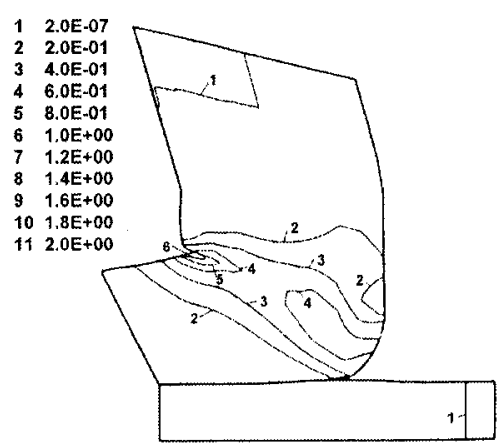

(a)

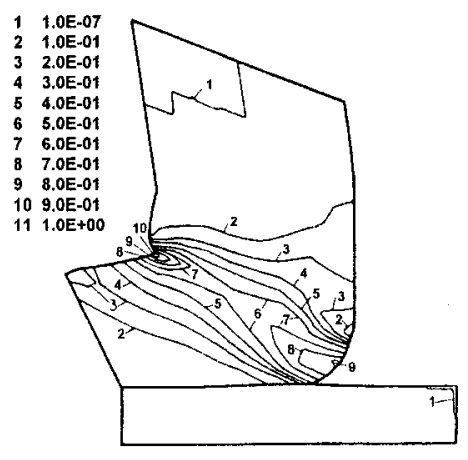

(b)

Figure 2.10: Distribution of strain in the elastic-plastic material with hardening and strain rate effects, for contact interface friction coefficients of (a) $\mu=0.0$ and (b) $\mu=0.15$; obtained from the work of Komvopoulos and Erpenbeck [41].

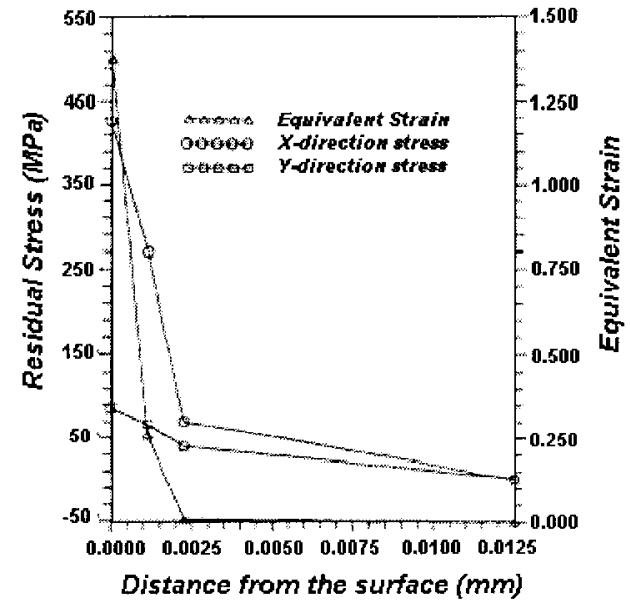

(a)

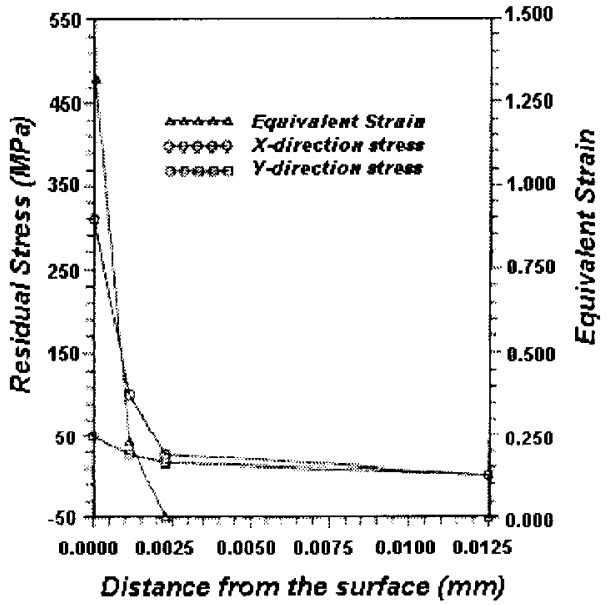

(b)

Figure 2:11: Variation of stress and strain within the machined surface for temperature being: (a) neglected, and (b) considered; obtained from the work of Lin and Lo [42]. 
but accounting for temperature effects decreased the stresses in the chip to between 200 and $450 \mathrm{MPa}$. Figure 2.11 shows the variation of stress versus distance from the machined surface for cases where temperature was neglected (Figure 2.11 (a)) and included (Figure 2.11 (b)). Temperature is shown to have little impact on equivalent strains, but has great impact on the stresses directly at the machined surface; at depths greater than $0.0025 \mathrm{~mm}$, the effect of temperature on stress becomes low. Lo and Lin [43] refined their model by defining a variable friction along the chip-tool interface, while keeping the remainder of the model consistent with their previous work [42]. The sticking region was determined by the shear stress experienced in the contacting elements; those elements with shear stress above yielding were assigned to the sticking region, while those below yielding were in the sliding region with a constant friction coefficient obeying Coulomb's law. Despite the improvements in modeling, Lin and Lo [43] were still lacking the experimental-numerical correlation to validate their model.

The model developed by Zhang and Bagchi [44] had a friction definition and separation criterion much like that of Lin and Lo [43], but used a much less extensive material model, which defined the behaviour of the workpiece as true stress-strain curve for strains ranging between 0 and 3. Despite the less precise material model definition, Zhang and Bagchi [44] had a more dependable model due to an extensive experimental-numerical correlation; experimental-numerical comparisons were made on the basis of cutting forces, primary shear angle measurements, and stresses. The assumptions in this model included the application of plane strain conditions and that the process was quasi-static. The numerical cutting forces showed a maximum relative difference of $20 \%$ from experimental forces. The primary shear angle calculated for OFHC copper, from the rake angle and feed-to-chip ratio, was within $8 \%$ of the experimentally measured primary shear angle of 28.2 degrees for the same cutting conditions. The numerical stress distributions were correlated to hardness maps derived from an experimental relation curve between strain and hardness, and showed a good agreement in distributions.

A physical criterion has also been successfully used in literature by Shet and Deng [45]. A model for the orthogonal cutting of AISI 4340 steel was developed using the FE code ABAQUS, assuming plane strain conditions. The workpiece material model 
included work hardening, strain rate and temperature effects. The tool was defined as a stiff elastic material, by setting the Young's modulus to an artificially high value. A single friction coefficient was defined along the rake face, however the fictional stress at the contact interface was equated to the lesser of the following two values: the shear failure stress of AISI 4340 or the normal pressure multiplied by the coefficient of friction. Overall, four different coefficients of friction were studied in this research: $0.0,0.2,0.4$ and 0.6. The separation of nodes along the cutting line was dependent on the stress and strain values accumulated at the nodes, and the failure tensile stress (948 MPa) and failure shear stress (548 MPa) defined for the workpiece material. The presented results included the distributions of temperature, von Mises stress, mean stress, normal strain, shear strain, and strain rate, for each of the studied coefficients of friction. Figure 2.12 illustrates the temperature distributions, and a temperature increase around the tool tip and adjacent to the rake face as the friction coefficient increased; the maximum temperature of $367^{\circ} \mathrm{C}$ for $\mu=0.0$ increased to $537^{\circ} \mathrm{C}$ for $\mu=0.6$, both occurring along the rake face. The magnitudes of strain and stress decreased at the rake face and at the tool tip due to material softening brought on by the temperature rise. Strain rates in the order of $10^{4}$ were concentrated in the PDZ. The only conclusions drawn from the results presented were that friction applied at the tool-chip interface has a large effect on the thermomechanical fields. No experimental correlation was provided for any of the results presented; therefore the model was lacking in validation.

The benefit of using the physical criterion in combination with the geometrical criterion was exemplified by the work of Obikawa et al. [46], who modeled the discontinuous chip formation in brass. The separation of the chip from the machined surface was still modeled through placing the geometrical criteria along the cutting line; however, a physical criteria was placed on all the nodes within the chip to enable the separation of nodes at a critical plastic strain and hence to model segmented chip flow. The accuracy of numerical predictions in experimental chip formation and separation is shown in Figure 2.13 for brass with composition of $62.8 \% \mathrm{Cu}$ and $37.2 \% \mathrm{Zn}$. The model was also verified by good correlation between numerical and experimental cutting forces, with $20 \%$ error. 


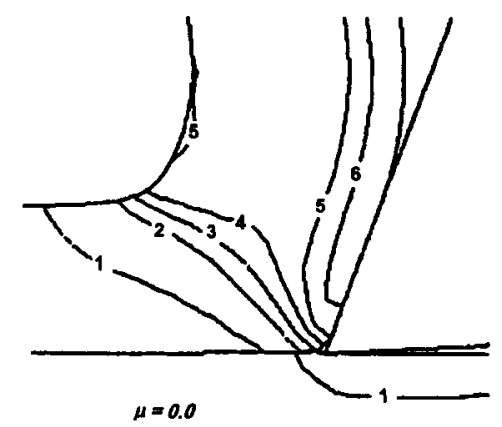

(a)

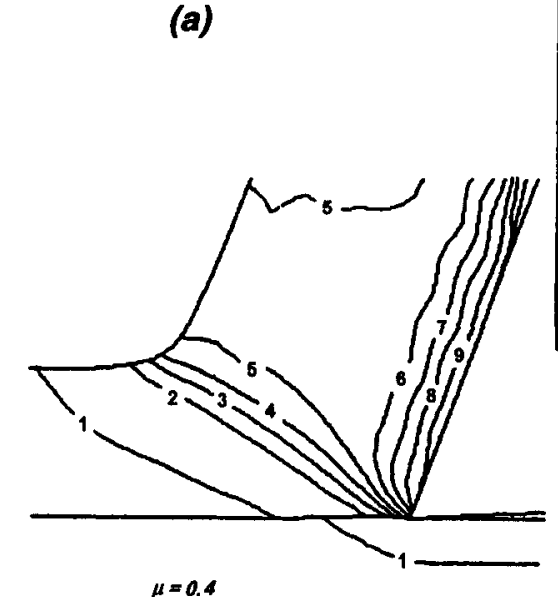

(c)

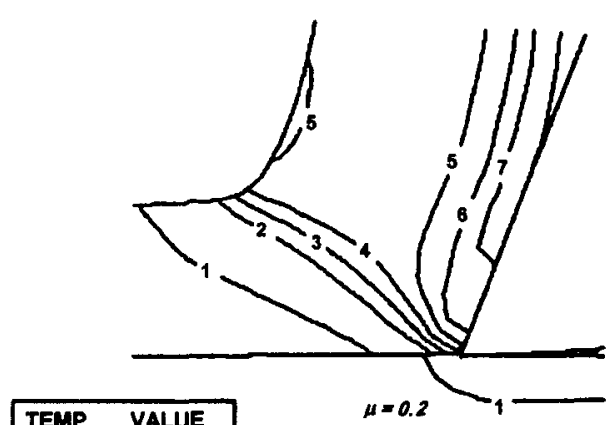

(b)

Figure 2.12: Temperature distribution obtained from a numerical model of AISI 4340 steel, for interface friction coefficients of: (a) $\mu=0.0$, (b) $\mu=0.2$, (c) $\mu=0.4$, and (d) $\mu=0.6$; obtained from the work of Shet and Deng [45].

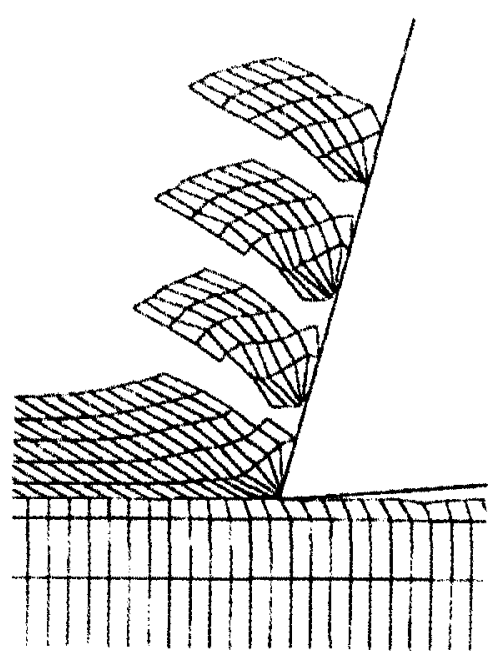

(a)

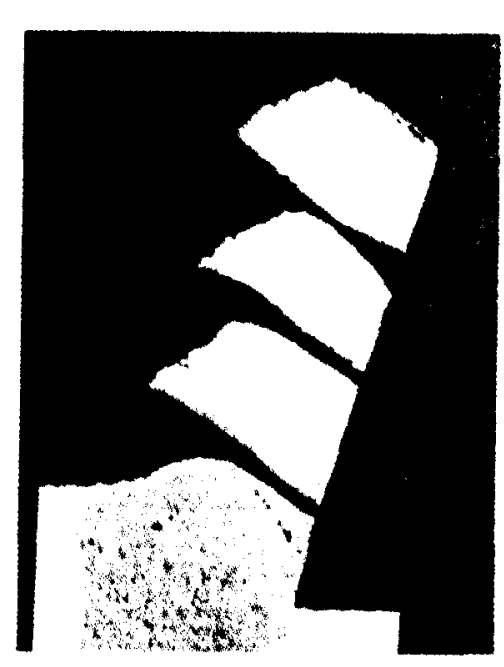

(b)

Figure 2.13: Comparison of the discontinuous chip formation in brass, predicted by (a) FE modeling, and (b) experimental tests; obtained from the work of Obikawa et al. [46]. 
The studies of Huang and Black [47] evaluated physical, geometrical, and a combination of physical and geometrical criteria. The geometrical condition was based on the critical distance of the nodes relative to the tool tip; the physical condition was in terms of the maximum shear stress experienced by the nodes; and the combination applied both criterion simultaneously with nodes separating when either of the conditions were satisfied. The analysis was performed on the NIKE2D FE code, which analyzes the deformation of two-dimensional, axisymmetric, plane stress and plane strain solids. The mild steel workpiece was modeled as an elastoplastic material with nonlinear strain hardening. Since focus was placed on examining the effectiveness of separation criteria, the chip-tool interface friction was neglected in this study. The values for the geometrical and physical separation criteria were determined through trial and error, and varied between $12 \mu \mathrm{m}$ and $48 \mu \mathrm{m}$ (for the geometrical definition) and $0.34 \mathrm{GPa}$ and $0.4 \mathrm{GPa}$ (for the physical definition). The separation criteria did not have an effect on chip geometry or distribution of stress, but it did have a significant effect on the magnitude of stress and the mesh distortion. The conclusion drawn from this research was that the combination of geometrical and physical criterion accurately predicts incipient as well as steady state chip formation, and is superior to either one used on its own.

While the Lagrangian mesh is capable of modeling metal cutting with the assistance of a failure criterion, the application of this approach approximates material fracture rather than the actual behaviour of material deformation. Adapting the mesh presents a viable alternative to the separation criterion and enables the modeling of material deformation and flow around the tool rather than fracture. Ceretti et al. [48, 49] used the commercial code DEFORM 2D to simulate the metal cutting process using both mesh smoothing and remeshing algorithms. The workpiece material of this model incorporated strain, strain rate, and temperature dependence. Details of the friction model were not discussed in references [48] and [49]. In addition to the measures taken to counteract the mesh distortion, a damage value was also assigned to elements, which were deleted upon exceeding this value to ease the chip and machined surface separation. The damage value was determined from information gathered through a tensile test, such as the effective stress and strain, maximum strain, and failure strain. Clearly the deletion 
of elements is a violation of the continuity law due to loss of volume, therefore the elements around the tool tip where deletion was most probable, were refined to minimize volume loss as shown in Figure 2.14. The algorithm was unable to contain the element deletion to the tool tip region. On occasion, elements in other areas met the criteria for deletion; and since they may have been considerably larger in size, volume loss was no longer insignificant. In addition to the volume loss, the downfalls of the model were lack of experimental verification and necessity of determining the damage value, which is unique to every type of workpiece material and operation parameters.

Continuous remeshing was also applied by Marusich and Ortiz [50], in modeling of AISI 4340 steel. The model was contained in two dimensions by applying plane strain conditions and the tool was modeled as rigid. The model was extensive in incorporating thermal softening, heat conduction, variable contact friction, and crack propagation leading to fracture. Mesh refinement was applied only to elements, which exceeded a critical value of plastic power density. In order to model the discontinuities in chip formation of AISI 4340 steel, a separation criterion was applied to the nodes in the chip, directing them to split upon reaching a critical effective strain dependent on the fracture toughness of the material. The temperature contours show a concentration of temperature increase at the contact interface of up to $990^{\circ} \mathrm{C}$ due to friction, and an increase in the PDZ of temperatures up to $500^{\circ} \mathrm{C}$ due to material shearing. The only remark made towards validation of the model was that numerical chip morphologies were in approximate range of experimental observations.

The major downfall of continuous mesh adaptivity is that it may not necessarily aid in decreasing error caused by mesh distortion, and yet the cost in computation time is high due to small incremental time steps required for proper mesh adaptivity.

\subsubsection{ALE Element Formulation}

Recently, the use of ALE element formulation has become more frequent in modeling high deformation problems such as machining. Benson [51] was one of the first researchers to introduce the concept of the ALE element formulation and discuss its strengths and weaknesses. One of the advantages of using this formulation is that it combines the finest characteristics of both the Lagrangian and Eulerian mesh [52], while 


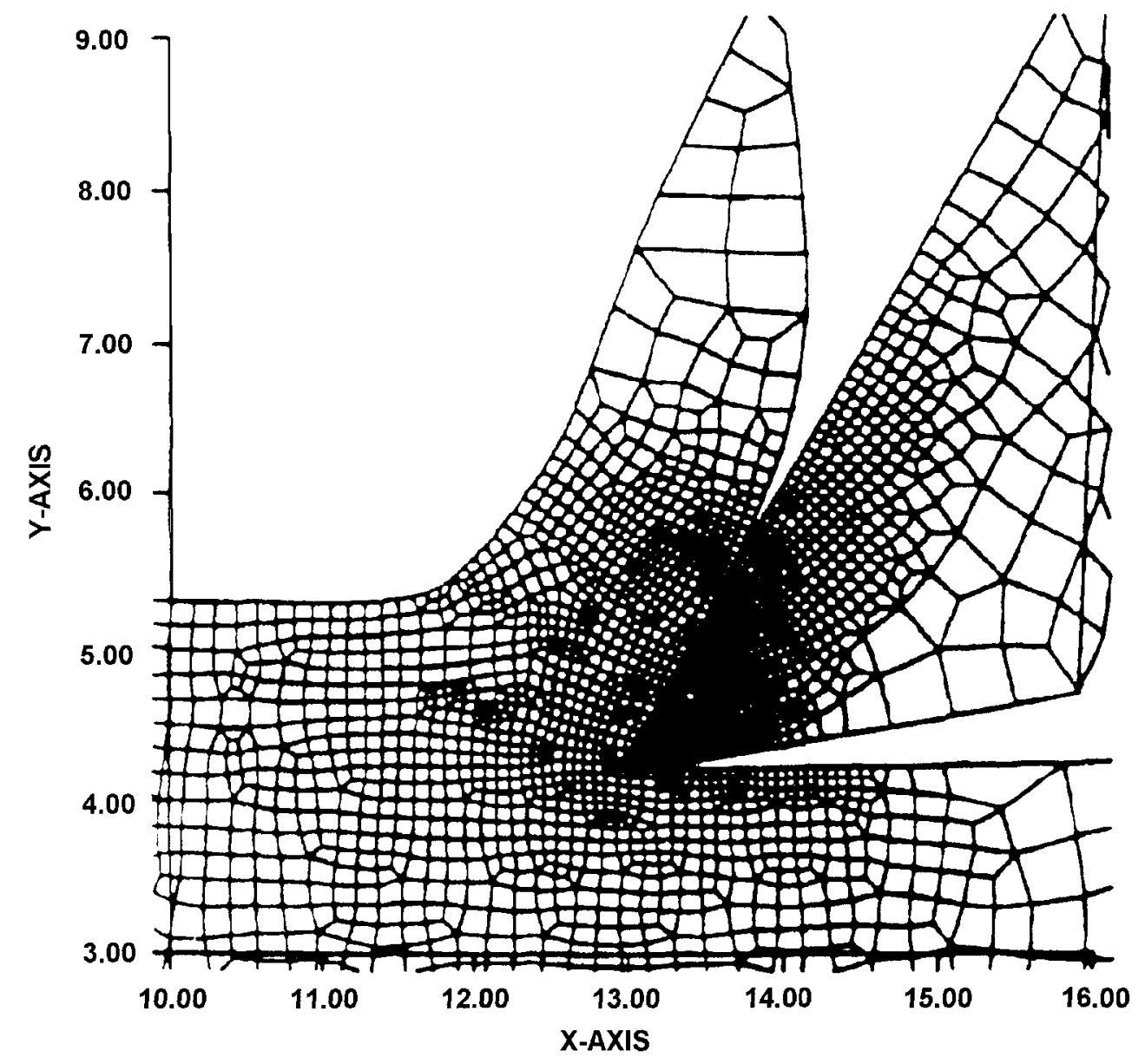

Figure 2.14: Mesh distribution in the workpiece and tool used in the FE modeling of Ceretti et al. [48]. 
being fairly simple to activate. An ALE mesh is able to combine the ability of an Eulerian mesh to withstand large deformation, while maintaining the ability of the Lagrangian mesh to track changes in material properties pertinent to solid mechanics. Researchers have been hesitant in applying hydrocodes to solid mechanics problems because they were designed to model fluid interaction, and their accuracy in modeling solid mechanics was uncertain.

Souli et al. [53] applied the ALE element formulation to model the Taylor bar impact test, and was able to duplicate Lagrangian mesh results with more ease and within half of the Lagrangian computation time; ultimately the numerical analysis lacked experimental comparison. The applicability of the ALE element formulation to metal forming problems was studied by Gadala and Wang [54], who applied the formulation to several high deformation solid mechanics problems, including: punch indentation, sheet metal extrusion, and compression between wedge-shaped dies. Each of the processes was successfully modeled and was in good agreement with experimental findings and theoretical estimations. Results from Lagrangian models of the same processes were compared to those of the ALE element formulations. General conclusions showed that ALE element technology is capable of modeling solid mechanics problems as well as the Lagrangian method, and better then the Lagrangian method in high deformation problems.

Movaheddy et al. [55] modeled metal cutting using the ALE element formulation. The process was not modeled from its steady state, as the initial workpiece geometry included an already formed chip with established chip thickness. With the ALE element formulation being incapable of supporting the extreme material deformation in the tool tip region, an Eulerian mesh was used adjacent to the rake face and around the tool tip. The workpiece material was not identified, but was simply modeled as an elastoplastic material with Young's modulus of $200 \mathrm{GPa}$, yield stress of $414 \mathrm{MPa}$ and strain hardening modulus of $1 \mathrm{GPa}$; further, the tool was assumed to be rigid, and the details of chip-tool interface friction were not discussed. The mesh distribution during chip formation is illustrated in Figure 2.15 with the circled Eulerian mesh at the rake face and around the tool tip. A stress distribution generated by the simulation is presented in Figure 2.16, and shows concentration of high stress in the PDZ, as well as increased stresses at 


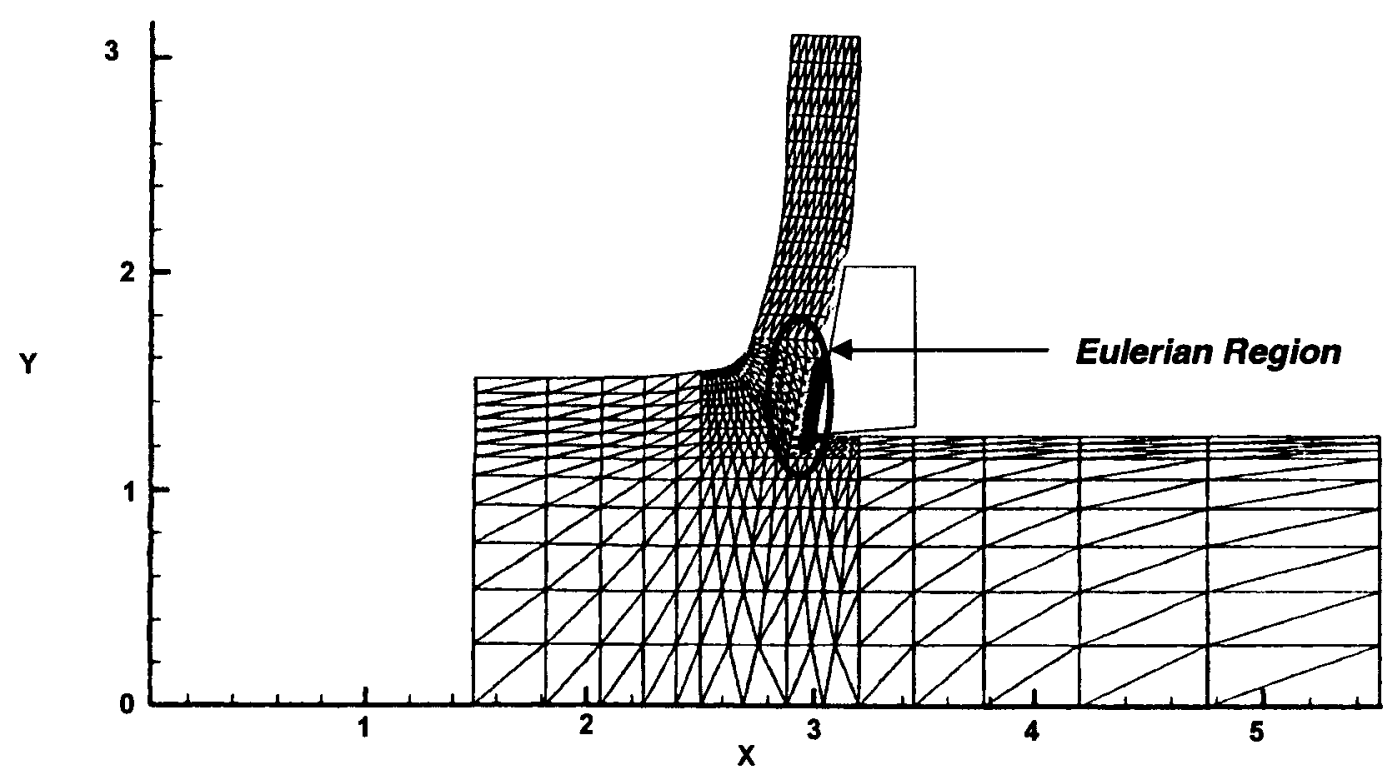

Figure 2.15: Mesh distribution in FE modeling of chip formation by Movaheddy et al. [55]; Eulerian region located adjacent to the rake face, is specified.

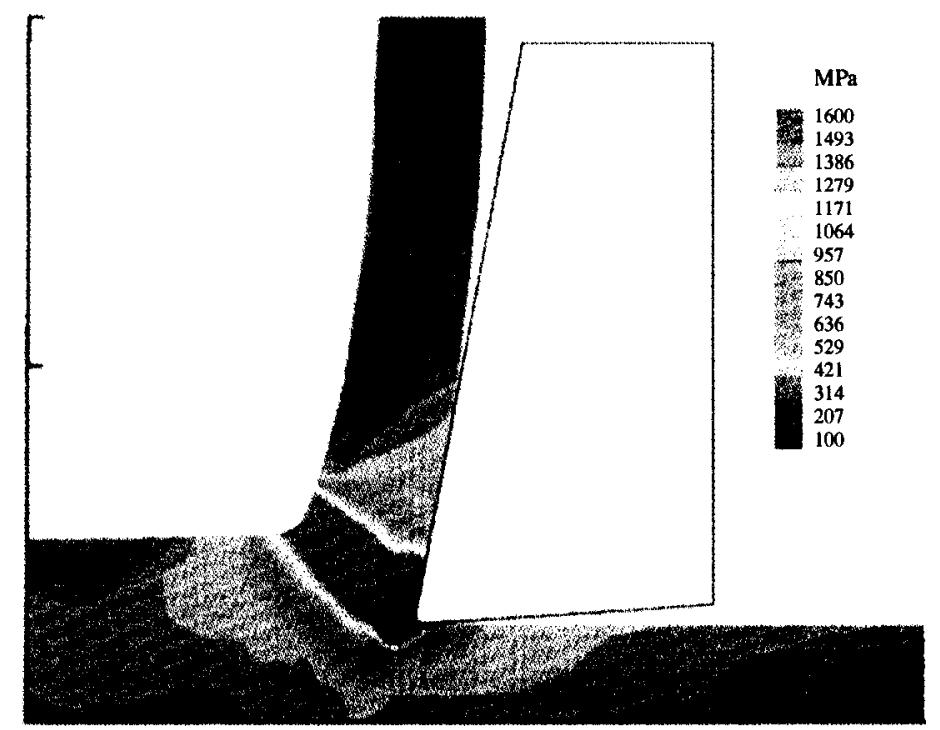

Figure 2.16: Stress distribution corresponding to chip formation shown in Figure 2.15; obtained from the work of Movaheddy et al. [55]. 
the tool rake face. The stress levels are fairly high, reaching values of $1600 \mathrm{MPa}$, but the values reflect the stress-strain relationship defined by the workpiece material model. Using the ALE formulation in combination with the Eulerian element formulation has enabled the chip formation to be modeled as a continuous flow of material around the tool tip (without a separation criterion being defined). One weakness in the model built by Movaheddy et al. [55] was that it was lacking any type of numerical-experimental correlation for validation of results.

\subsubsection{Eulerian Element Formulation}

The Eulerian element formulation has remained virtually uncultivated in FE modeling of machining. Only recently has the modeling of solid mechanics problems using an Eulerian element formulation been explored [23]. Although forfeiting the Lagrangian element formulation for the Eulerian element formulation gives rise to some disadvantages, it is beneficial especially in high deformation manufacturing processes. It is more difficult to track time dependent material properties in the Eulerian mesh because the mesh in not attached to the material. Conversely, the Eulerian mesh remains undistorted regardless of the degree of material deformation, and its accuracy does not diminish. It is therefore a good candidate for studying high deformation processes such as metal cutting. 


\subsection{EXPERIMENTAL DATA USED FOR MODEL VERIFICATION IN THE PRESENT WORK}

Extensive experimental observations were performed by Elmadagli and Alpas [56], on the workpiece material directly ahead of the tool tip. The extent of the information obtained during this research has the capability of verifying the results of the numerical model, and was therefore used extensively during all phases of numerical modeling. Due to the importance of the work of Elmadagli and Alpas [56] to the presented research, the following is a summary of their published work.

The investigated material was commercial purity ETP Copper (C11000), with properties listed in Table 2.1. The sample was in the form of a hot extruded rod with $25.4 \mathrm{~mm}$ diameter; the rod was machined into a tubular shape with $3.0 \mathrm{~mm}$ wall thickness prior to the orthogonal cutting tests. The grain size was calculated according to ASTM E 112-96 [57], while the hardness was measured by a Knoop indenter with a load of $245 \mathrm{mN}$; both are listed in Table 2.1 .

\begin{tabular}{|c|c|}
\hline Material Property & Value with units \\
\hline Density $(\rho)$ & $8.9 \mathrm{~g} \cdot \mathrm{cm}^{-3}$ \\
\hline Young's Modulus $(E)$ & $115.0 \mathrm{GPa}$ \\
\hline Poisson's Ratio $(v)$ & 0.33 \\
\hline Yield Strength $\left(\sigma_{y}\right)$ & $199.3 \mathrm{MPa}$ \\
\hline Shear Modulus $(G)$ & $47 \mathrm{GPa}$ \\
\hline Melting Temperature $\left(T_{\text {mell }}\right)$ & $1065^{\circ} \mathrm{C}$ \\
\hline Specific Heat $\left(C_{p}\right)$ & $384.7 \mathrm{~J} \cdot \mathrm{kg}^{-1} \cdot{ }^{\circ} \mathrm{C}^{-1}$ \\
\hline Grain Size & $47.4 \mu \mathrm{m}$ \\
\hline Hardness $(H)$ & $67.4 \mathrm{~kg} \cdot \mathrm{mm}^{-2}$ \\
\hline
\end{tabular}

Table 2.1: Properties of the commercial purity copper workpiece material in the experimental machining tests, as measured experimentally and as reported in the ASM Metals Handbook: Volume 2 [58].

Orthogonal cutting tests were performed on a standard lathe (Harrison M3000, TS Harrison \& Sons, West Yorkshire, UK), without the use of cutting fluids. SiAlON grade Silicon Nitride based ceramic cutting tool inserts (Kennametal CNGA-432 [59]) with a negative 5 degree rake angle were used to cut the work material, the properties of 
the tool are listed in Table 2.2. A cutting speed of $0.6 \mathrm{~m} \cdot \mathrm{s}^{-1}$ and feed rate of $0.25 \mathrm{~mm}$ were used in the cutting process

\begin{tabular}{|c|c|}
\hline Material Property & Value with units [3] \\
\hline Density $(\rho)$ & $3.24 \mathrm{~g} \cdot \mathrm{cm}^{-3}$ \\
\hline Young's Modulus $(E)$ & $288.0 \mathrm{GPa}$ \\
\hline Poisson's Ratio $(v)$ & 0.25 \\
\hline
\end{tabular}

Table 2.2: Properties of SiAION grade Silicon Nitride tool inserts in the experimental machining tests, obtained from ASM Metals Handbook: Volume 16 [3].

A three component piezoelectric dynamometer was used to measure the resultant force acting on the tool by its two components; the cutting force $\left(F_{c}\right)$ along the cutting direction, and the thrust force $\left(F_{t}\right)$ perpendicular to the cutting direction. The steady state cutting force and the thrust force were measured to be $1177.2 \mathrm{~N}$ and $945.7 \mathrm{~N}$, under the cutting conditions employed. The cutting process was stopped using a rapid action foot controlled brake with electrical disengagement in order to produce chips attached to the workpiece. Interrupted cut specimens were sectioned for metallographic examination of their deformation microstructures. Samples were prepared using standard metallographic preparation techniques and etched with a solution composed of five parts of $\mathrm{HNO}_{3}$ and acetic acid, and one part of $\mathrm{H}_{3} \mathrm{PO}_{4}$.

The deformation microstructure of the sectioned C11000 interrupted cut specimen, is shown in Figure 2.17. The nearly equiaxed grains of the workpiece, became elongated in the direction of deformation assuming a 'tear drop' shape within the PDZ, where grain axes became aligned in the plastic flow direction. The direction of the plastic flow at each point is represented by the value of the shear angle $(\phi)$, indicated in Figure 2.18. The values of local equivalent strains $\bar{\varepsilon}^{p}$ in the material ahead of the tool tip were estimated using [60-64]:

$$
\bar{\varepsilon}^{p}=\frac{\sqrt{3}}{3} \tan \theta
$$

where the deformation angle $\theta$ is $\left(90^{\circ}-\phi\right)$. 

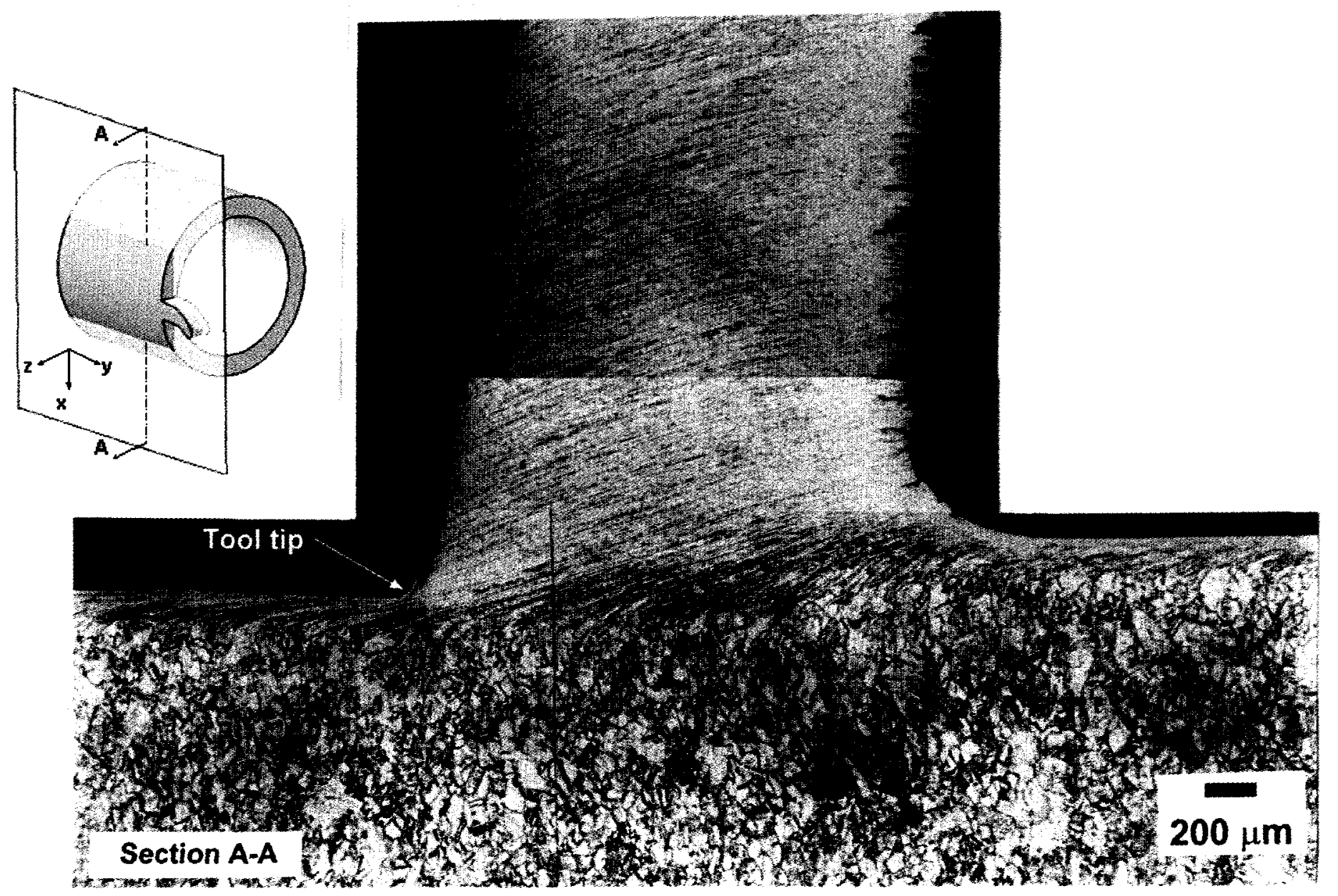

Figure 2.17: Optical microstructure of workpiece material ahead of the tool tip, obtained from the cross-section (AA shown in the insert), of a commercial purity copper sample, which has undergone orthogonal cutting. Cutting speed $v_{c}=0.6 \mathrm{~m} / \mathrm{sec}$, feed rate $f=0.25 \mathrm{~mm} / \mathrm{rev}$ and tube wall thickness $w=3.0 \mathrm{~mm}$ in accordance to the work of Elmadagli and Alpas [56]. 
Microhardness measurements were taken at intersection points of an imaginary $225 \mu \mathrm{m} \times 125 \mu \mathrm{m}$ grid within the deformed material ahead of the tool tip. The indentations were made by a Knoop indenter with a load of $245 \mathrm{mN}$ and with the long diagonal of the indenter parallel to the cutting direction. Two additional measurements were taken at $30 \mu \mathrm{m}$ above and below each indentation point, and the mean value of these measurements was used to represent local microhardness. The variation of microhardness of the material ahead of the tool tip is presented in Figure 2.19. Local flow strength values of the material were estimated using the following equation [65].

$$
\sigma=\frac{H}{C}
$$

where $\sigma$ is the flow stress, $H$ is the microhardness, and the constant (constraint factor) $C=3.0$. This equation was commonly used for determination of flow strength values of pre-strained materials for which direct testing is not practical.

Corresponding stress and strain values determined at each point in the material ahead of the tool tip were plotted to obtain a relationship between the equivalent stresses and strains. The resulting flow curve is shown in Figure 2.20. The material shows rapid strain hardening as depicted in the initial section of the flow curve, followed by the inability of the material to strain harden further where a saturation stress is reached. A regression analysis showed that this curve could be described in the form of a Voce type exponential relationship [66].

$$
\sigma=\sigma_{s a t}-\left(\sigma_{s a t}-\sigma_{o}\right) \exp \left(\frac{-\bar{\varepsilon}^{p}}{\varepsilon_{c}}\right)
$$

where $\sigma$ is the value of the equivalent flow stress, $\bar{\varepsilon}^{p}$ is the corresponding equivalent plastic strain, $\sigma_{s a t}(422.2 \mathrm{MPa})$ is the saturation stress or the stress at which the work hardening rate becomes zero, $\sigma_{o}(199.3 \mathrm{MPa})$ is the flow strength of the material determined by microhardness measurements, and $\varepsilon_{c}(1.89)$ is a constant.

Stress and strain maps will be presented in latter sections, along with the numerical results for correlation and discussion purposes. 


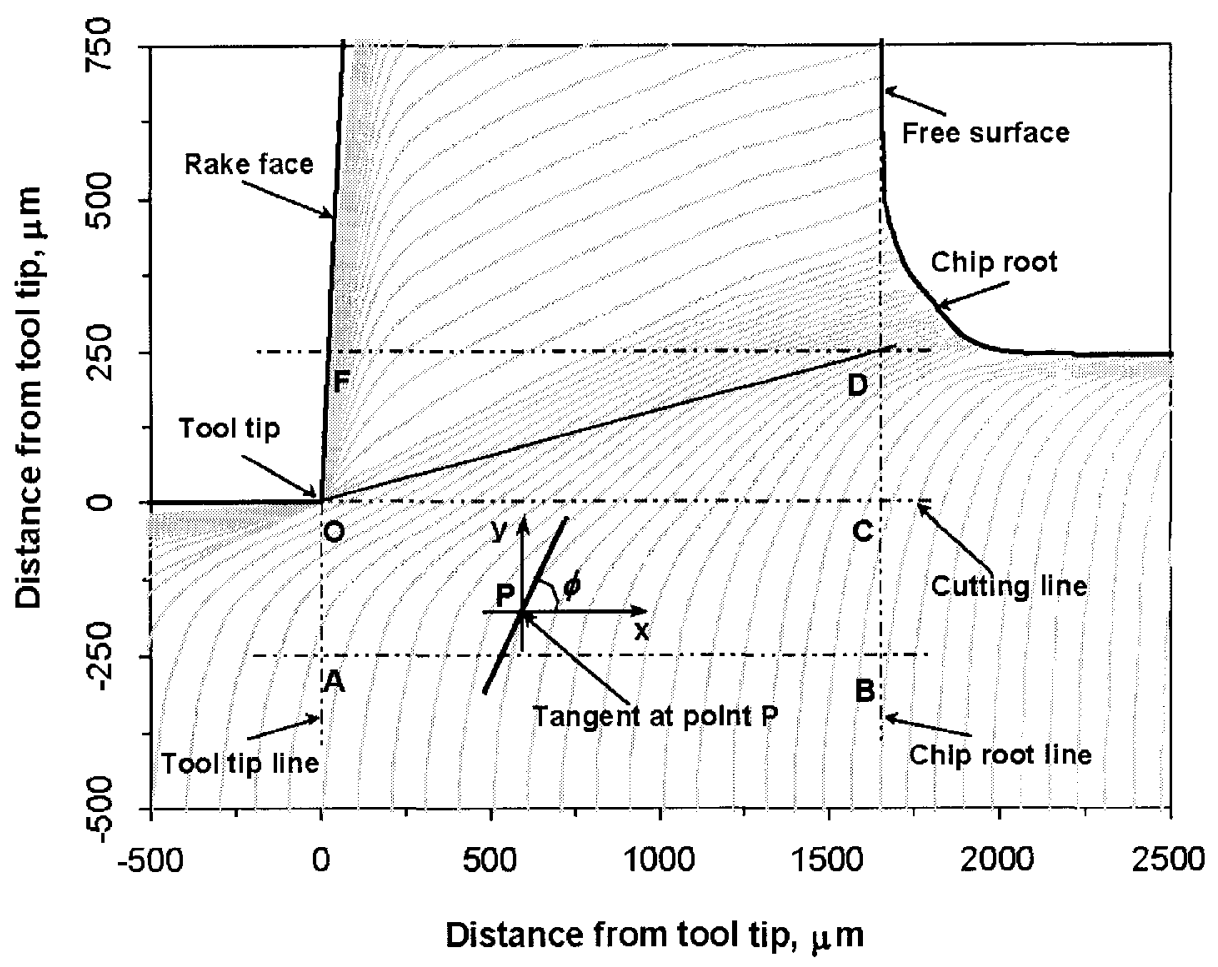

Figure 2.18: Flow lines defining deformation patterns of microstructure in Figure 2.17, constructed by Elmadagli and Alpas [56] by outlining orientation changes in grain boundaries. The cutting line and primary shear plane are labelled as OC and OD, respectively.

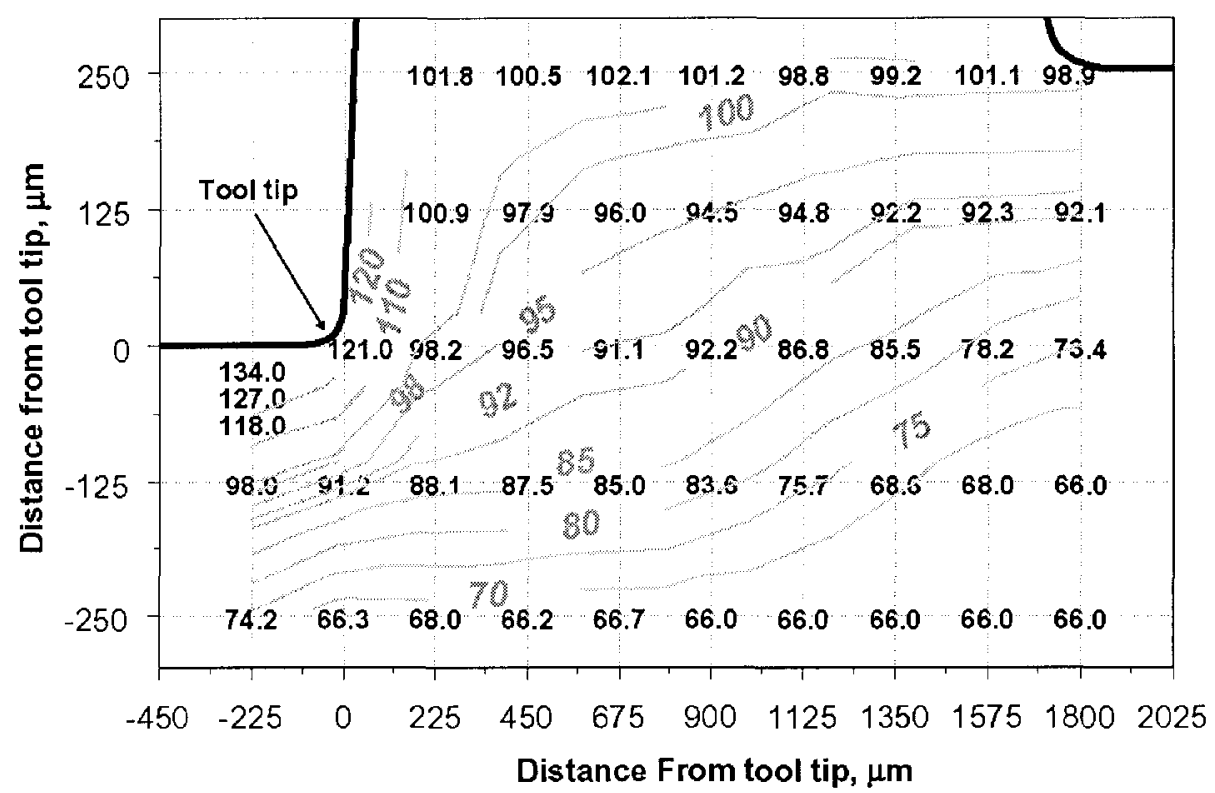

Figure 2.19: Microhardness distribution in the machined sample showing local microhardness values taken at intersections of a $225 \mu \mathrm{m} \times 125 \mu \mathrm{m}$ grid and lines of constant hardness contours, obtained from the work of Elmadagli and Alpas [56]. 


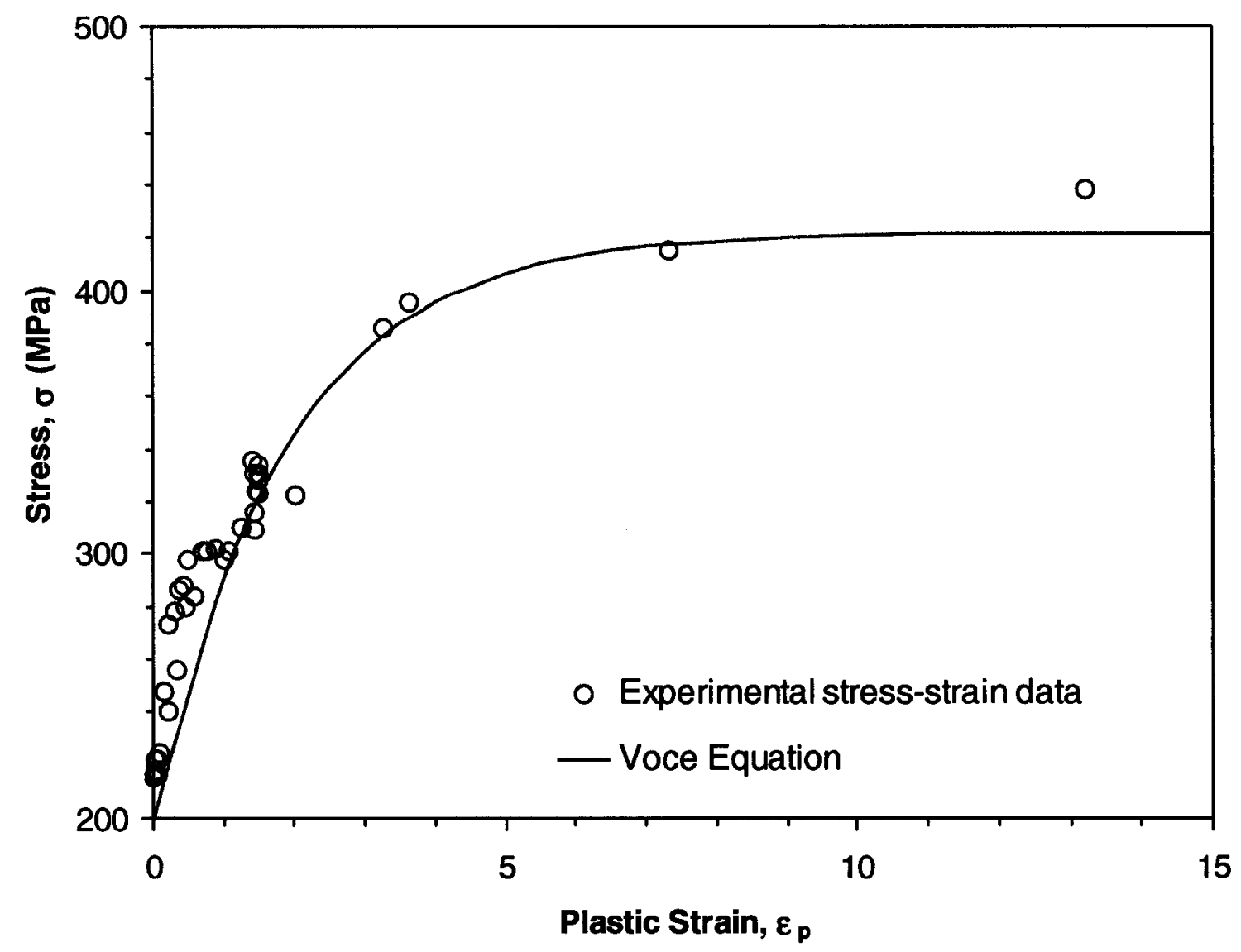

Figure 2.20: Cumulative stress-plastic strain flow curve of copper and experimental stress and strain values corresponding to specific locations in the material ahead of the tool tip, reported by Elmadagli and Alpas [56]. 


\section{CHAPTER 3}

\section{Research Focus}

All three element formulations available in the FE code LS-DYNA were studied in the scope of this project for their applicability to the modeling of metal cutting processes. The Lagrangian and ALE element formulations have been used in previous research to model metal cutting, and have proven to lack the capabilities to simulate the excessive material deformation required to displace the workpiece at the tool tip to form a chip. Experimental-numerical comparisons were rarely performed for the Lagrangian and ALE results analyses because the mesh distortion incurred inaccuracies, which made correlation unfeasible.

In an effort to develop an innovative model, one that would surpass the capabilities of the existing models, the investigation of the Lagrangian and ALE element formulations was limited and focus was placed on employing the Eulerian element formulation to model chip formation.

The following were objectives of the present work:

- Build a FE model of the metal cutting process utilizing all three element formulations, with precedence given to the Eulerian element formulation.

- Refine the most promising model, by investigating the validity of different material models, including both Johnson-Cook as well as elastic plastic hydrodynamic constitutive models.

- Correlate the numerical result to the experimental observations to establish, which FE model best predicts the behaviour of commercial purity copper subjected to metal cutting. Experimental observations available for correlation include: strain distribution, stress distribution, cutting and thrust forces, and chip geometry. 


\section{CHAPTER 4}

\section{Finite Element Procedures I :}

\section{Metal Cutting Model Employing the Lagrangian Element Formulation}

This chapter summarizes the study of steady state chip formation using a Lagrangian element formulation. In summary, the following simulations were performed using this element technology:

- Mesh Sensitivity Analysis: Four numerical models were studied, each with a different level of mesh refinement in the PDZ of the workpiece (see Section 4.1).

- Full Mesh Adaptivity: The coarsest workpiece mesh was adapted in latter stages of the simulation to counteract mesh distortion (see Section 4.2)

- Partial Mesh Adaptivity: Small area of the coarsest mesh, adjacent to the tool tip, was adapted to eliminate severe mesh distortion (see Section 4.2).

Summary of the FE input file required for the mesh sensitivity analysis is contained in Appendix $\mathrm{C}$, while the input used in the mesh adaptivity studies are summarized in Appendices D and E, for full and partial adaptivity, respectively. The essential input components are explained in subsequent sections, while detailed description of all keywords can be found in the LS-DYNA Keyword User's Manual: Volumes I and II [30, 32].

The results of the simulations performed with the Lagrangian element formulation are presented in Chapter 7.

\subsection{MESH SENSITIVITY ANALYSIS}

\subsubsection{Geometry and Mesh Generation}

The geometry of the numerical model used to investigate the application of the Lagrangian element formulation was developed in FEMB and is shown in Figure 4.1. The complete model consisted of two parts, the workpiece ( $P I D=2$ on pg. 191) and the 


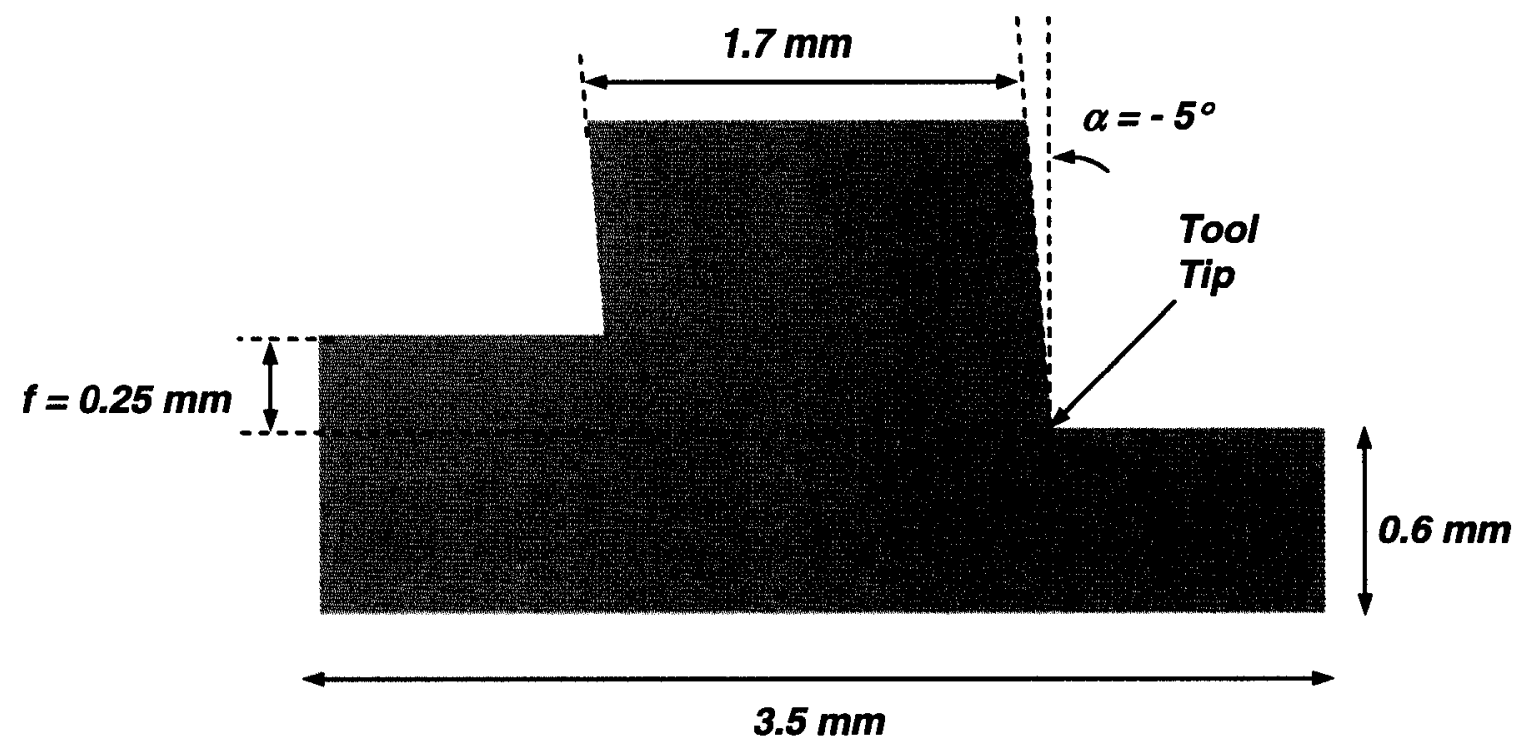

(a)
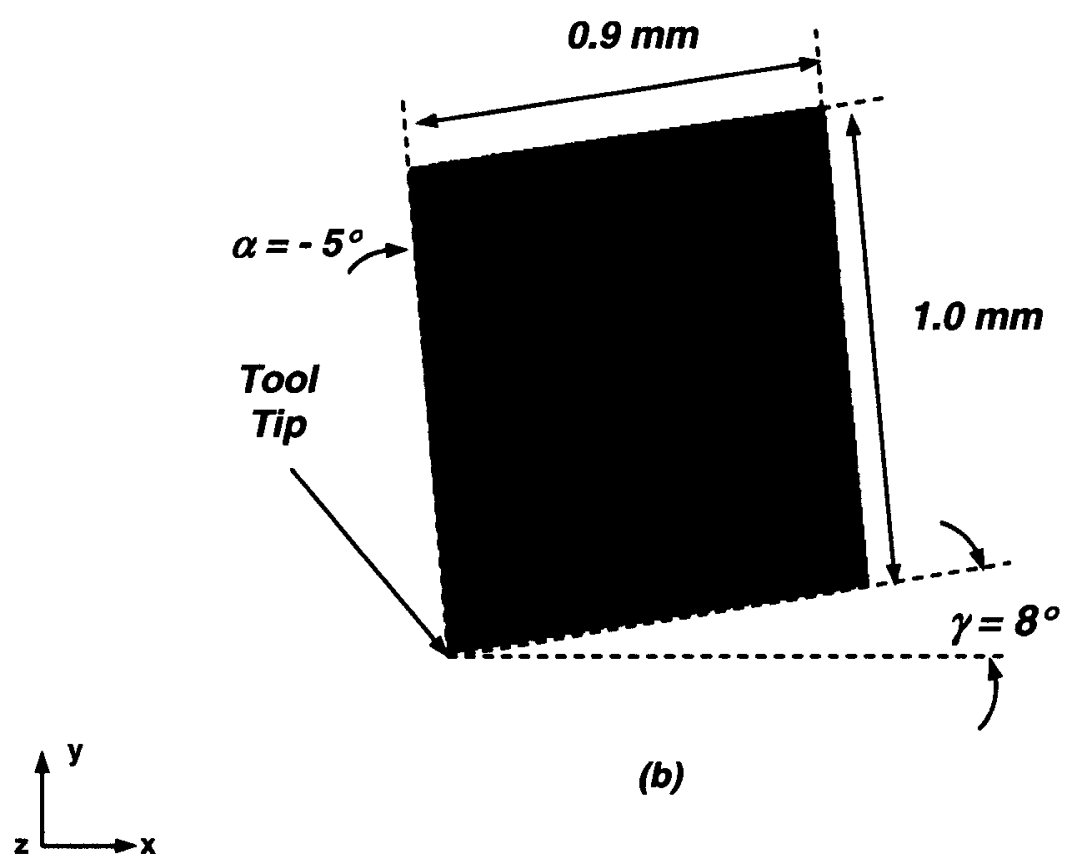

(b)

Figure 4.1: Geometry used in the Lagrangian element formulation for (a) workpiece, and (b) tool. 
tool ( $\mathrm{PID}=1$ on pg. 191). The cutting parameters were selected to duplicate the experimental investigations [56], with a feed $(f)$ of $0.25 \mathrm{~mm}$, clearance angle $(\gamma)$ of 8 degrees, and rake angle $(\alpha)$ of negative 5 degrees. The remainder of the dimensions were chosen to suffice accurate modeling of the process while keeping the number of elements low in efforts to reduce computation time.

Four models were developed to study the sensitivity of results on element size, and hence to find an optimal range of element size for the simulations. Each workpiece mesh had different levels of refinement in the PDZ and SDZ where deformation is the greatest. To ensure the most accurate contact algorithm execution, elements on the rake face of the tool, contacting the SDZ of the workpiece, were refined accordingly. Mesh (a) of Figure 4.2 was the first and coarsest mesh developed for the Lagrangian study, and was progressively refined to produce consecutive meshes (b) through (d). A summary of the number of elements and smallest elements size of each numerical model is given in Table 4.1. The information of each particular element is stored in the *ELEMENT_SHELL card (pgs. 192, 195 and 198), which includes the part number to which the element belongs (PID), and the four nodes that make up the quadrilateral element (N1, N2, N3, and N4). The nodes are defined by their coordinates (X, Y, and $\mathrm{z}$ ) in the * NODE card.

\begin{tabular}{|c|c|c|c|c|}
\cline { 3 - 5 } \multicolumn{1}{l|}{} & $\begin{array}{c}\text { Number of } \\
\text { Elements }\end{array}$ & $\begin{array}{c}\text { Minimum Element Size } \\
(\mu \mathrm{m})\end{array}$ & Element Aspect Ratio \\
\hline \multirow{2}{*}{ Mesh (a) } & Workpiece & 1015 & $60 \times 60$ & 1.0 \\
\cline { 2 - 5 } & Tool & 180 & $75 \times 67$ & 1.1 \\
\hline \multirow{2}{*}{ Mesh (b) } & Workpiece & 1792 & $30 \times 30$ & 1.0 \\
\cline { 2 - 5 } & Tool & 213 & $45 \times 38$ & 1.2 \\
\hline \multirow{2}{*}{ Mesh (c) } & Workpiece & 3181 & $15 \times 15$ & 1.0 \\
\cline { 2 - 5 } & Tool & 802 & $19 \times 20$ & 1.1 \\
\hline \multirow{2}{*}{ Mesh (d) } & Workpiece & 5358 & $7.5 \times 7.5$ & 1.0 \\
\cline { 2 - 5 } & Tool & 802 & $19 \times 20$ & 1.1 \\
\hline
\end{tabular}

Table 4.1: Number of elements and minimum element size in meshes (a) through (d) in the Lagrangian element formulation study. 


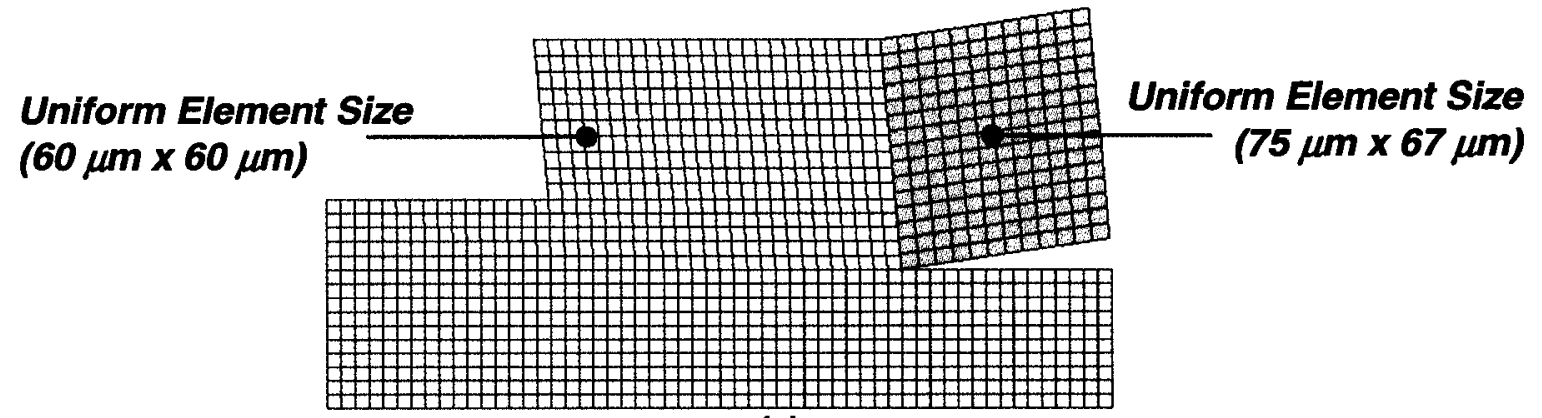

(a)

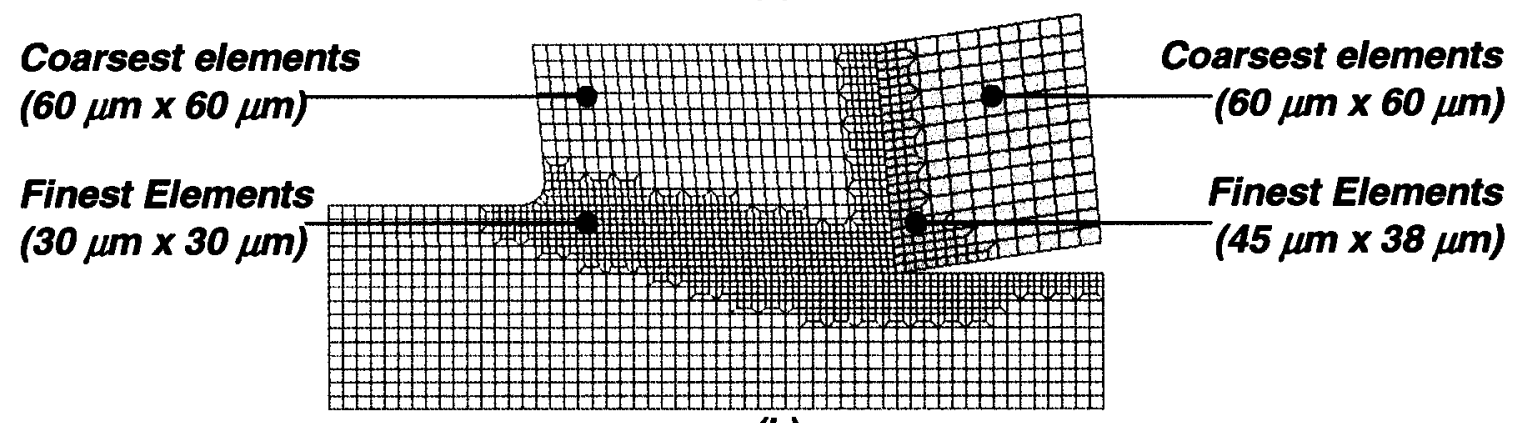

(b)

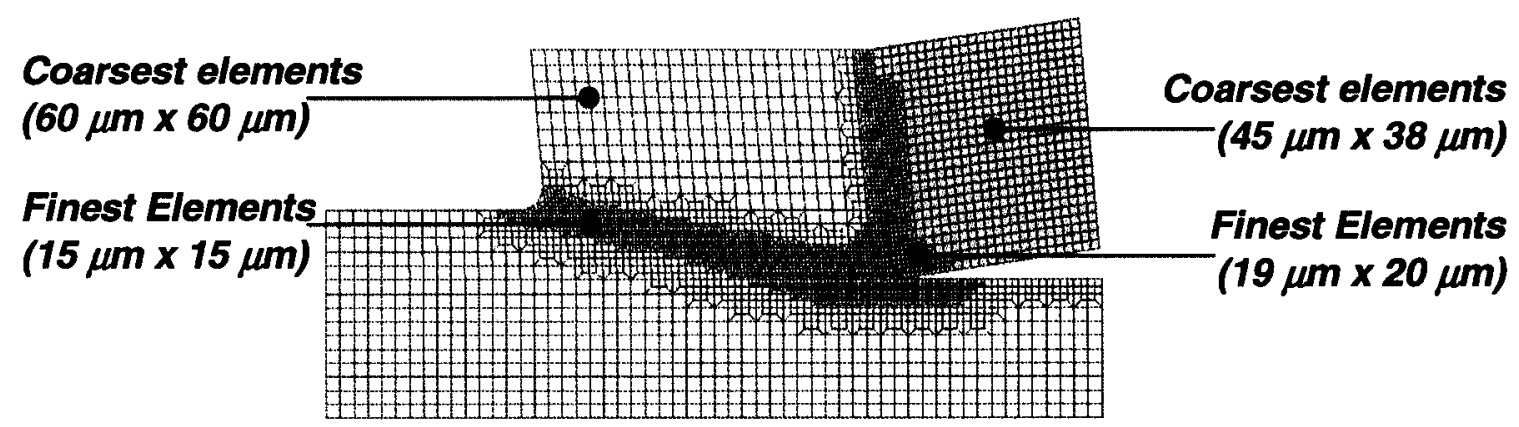

(c)

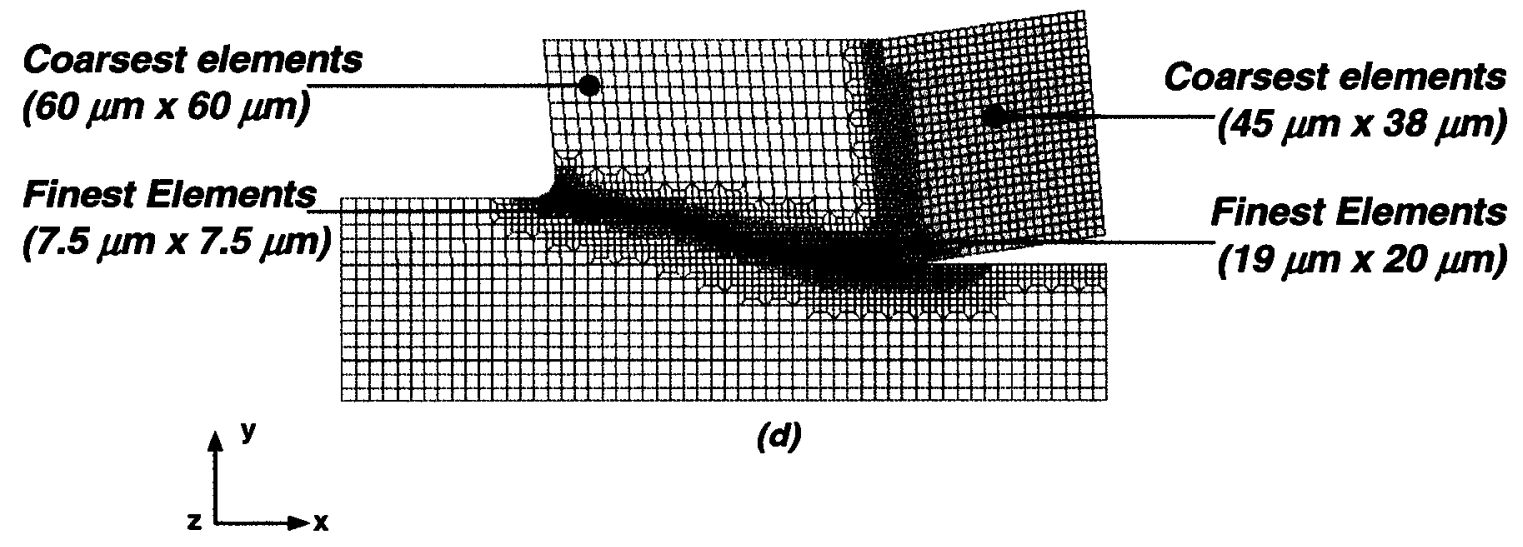

Figure 4.2: Meshes generated for each of the four models of the workpiece and tool with (a) uniform mesh, (b) tool and workpiece mesh refined in the PDZ and SDZ, (c) further mesh refinement in the PDZ and SDZ, (d) finest mesh in the PDZ and SDZ. Also noted are the coarsest and finest mesh locations. 


\subsubsection{Element Properties}

The ratio of experimental chip thickness in z-direction $(3.0 \mathrm{~mm})$ to feed $(0.25 \mathrm{~mm})$ exceeded 10.0 , which validates the application of plane strain conditions. The three-dimensional oblique process was reduced to two-dimensional orthogonal cutting in the numerical simulations. The plane strain properties were assigned in the *SECTION_SHELL card (pgs. 192, 195 and 198) by defining ELFORM=13. The workpiece and tool were modelled as shell elements in the xy plane, with an arbitrary thickness of $5 \mu \mathrm{m}$ defined at each of the four nodal points ( $T 1, T 2, T 3$ and $T 4$ ).

\subsubsection{Material Properties}

The material models chosen for the Lagrangian element formulation study were the Material type 24 (*MAT_PIECEWISE_LINEAR_PLASTICITY on pgs. 192, 195 and 198) for the workpiece and Material type $20\left({ }^{\star}\right.$ MAT_RIGID on pgs. 192, 195 and 198) for the tool.

Material type 24 enables the user to define the stress-strain relationship of a material by means of plastic strain $\left(\bar{\varepsilon}^{p}\right)$ values paired with corresponding yield stress values $(\sigma)$. Stress and strain data for commercial purity ETP copper undergoing machining presented in reference [56] is provided in the form of an exponential Voce equation described in Section 2.4 and Equation (2.23). Therefore Material type 24 was chosen to model the workpiece due to the ease of curve fitting representative points into the experimentally derived function. A load curve containing twenty-one points was used to characterize commercial purity ETP copper material behaviour. It was correlated through LCSS $=2$ in the material card and defined in *DEFINE_CURVE (pgs. 192, 195 and 198). The plastic strain values were distributed between 0.0 and 20.0 to ensure that all values, which could possibly arise in the simulation, would be accounted for. The corresponding stress values were calculated through the Voce equation derived in [56]. The stress-strain values are listed in Table 4.2 and are shown in Figure 4.3 in association with the Voce equation curve fit of experimental data. The additional properties of density, Young's modulus, Poisson's ratio, and yield strength were required to complete the model. These are provided in Table 2.1. 


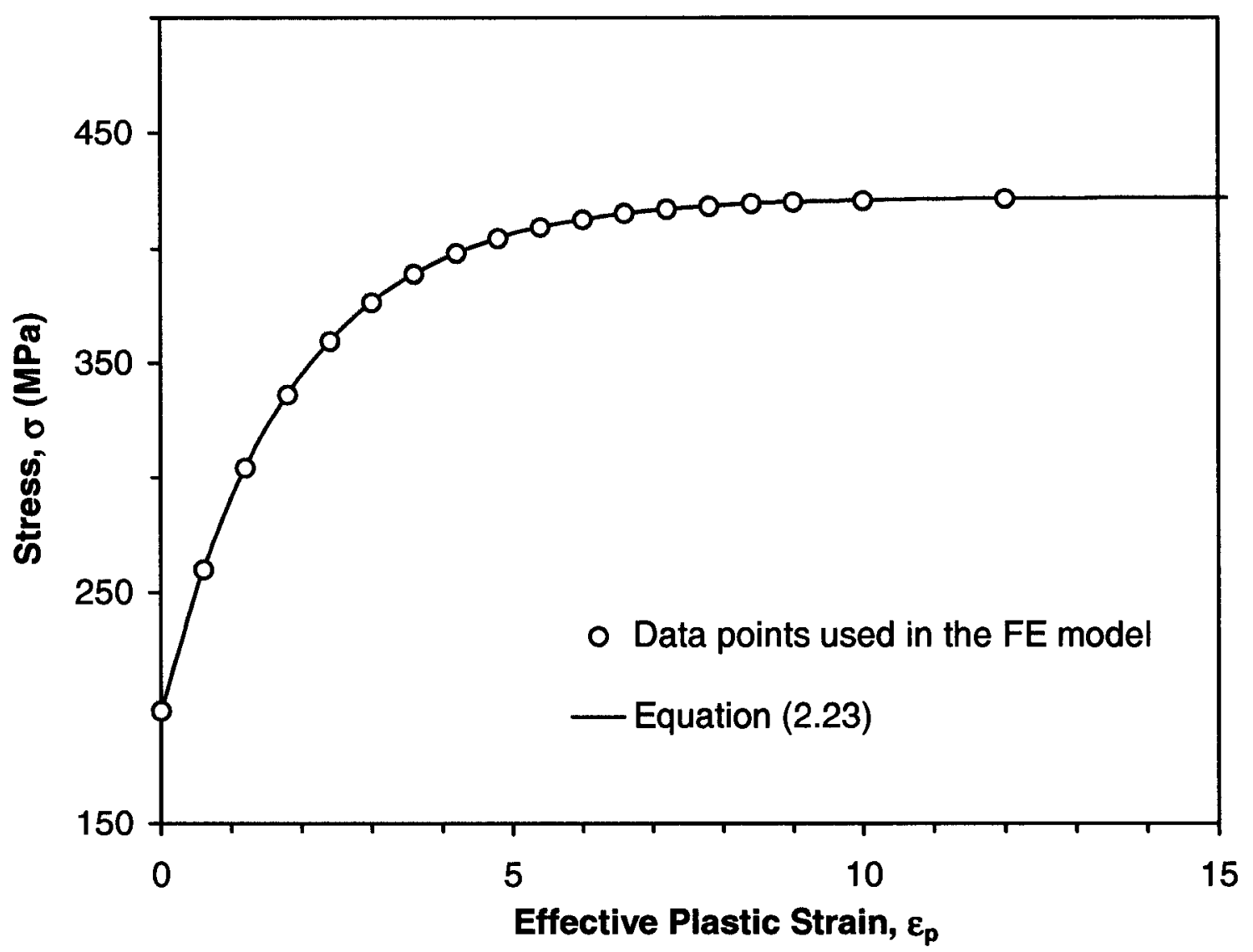

Figure 4.3: Stress-plastic strain behaviour of $\mathrm{C} 11000$ undergoing orthogonal cutting characterized by the Voce equation curve [56] and curve fitting points used in Material type 24. 


\begin{tabular}{|c|c|c|c|c|c|}
\hline$\varepsilon_{p}\left(\mathbf{m m} \cdot \mathbf{m m}^{-\mathbf{1}}\right)$ & $\sigma(\mathbf{M P a})$ & $\varepsilon_{p}\left(\mathbf{m m} \cdot \mathbf{m m}^{-1}\right)$ & $\sigma(\mathbf{M P a})$ & $\varepsilon_{p}\left(\mathbf{m m} \cdot \mathbf{m m} \mathbf{m}^{-\mathbf{1}}\right)$ & $\sigma(\mathbf{M P a})$ \\
\hline 0.0 & 199.30 & 4.2 & 389.04 & 8.4 & 419.58 \\
\hline 0.6 & 259.93 & 4.8 & 404.62 & 9.0 & 420.29 \\
\hline 1.2 & 304.07 & 5.4 & 409.40 & 10.0 & 421.08 \\
\hline 1.8 & 336.20 & 6.0 & 412.88 & 12.0 & 421.81 \\
\hline 2.4 & 359.59 & 6.6 & 415.42 & 16.0 & 422.15 \\
\hline 3.0 & 376.62 & 7.2 & 417.26 & 18.0 & 422.18 \\
\hline 3.6 & 389.01 & 7.8 & 418.60 & 20.0 & 422.19 \\
\hline
\end{tabular}

Table 4.2: Stress-plastic strain values used to characterize commercial purity ETP copper material behaviour for Material type 24.

The tool was modeled as a rigid material for the following reasons: 1) mechanical properties of the SiAlON grade tool exceed those of the commercial purity ETP copper workpiece, and 2) Material type 20 required far less computations during simulations thereby shortening the time to complete the simulation. Properties defined in *MAT_RIGID (pgs. 192, 195 and 198), included density, Young's modulus, and Poisson's ratio and are listed in Table 2.2 .

Because the tool was modeled as rigid, Young's modulus did not have any influence over the behaviour of the tool. The Young's modulus of the tool only contributed to the calculation of the contact spring stiffness defined by Equation (2-17). An extremely high $E$ value, such as that of the tool material, significantly raises the spring stiffness in contact and can result in unrealistic contact modeling; therefore Young's modulus for the tool was defined as $115.0 \mathrm{GPa}$.

\subsubsection{Boundary and Loading Conditions}

To properly model the experimental set-up, where one part is stationary while the other moves, the bottom surface of the workpiece was restricted in all degrees of freedom

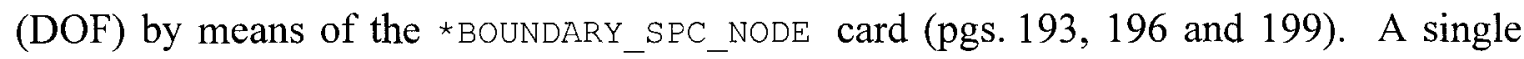
point constraint (SPC) was activated on listed nodes by placing " 1 " in each of the six columns representing the six DOF: DOFX ( $\mathrm{x}$ translation), DOFY (y translation), DOFZ (z translation), DOFRX (x rotation), DOFRY (y rotation), and DOFRZ (z rotation). 
The motion of the tool was generated by *LOAD_RIGID_BODY (pgs. 193, 196 and 199), with reference to a load curve (LCID=1) defined by *DEFINE CURVE (pgs. 193, 196 and 199). The load was applied in the $x$-direction (DOF=1). A constant load was applied throughout the duration of the simulation with ABCISSA defining the time ( 0 to $0.5 \mathrm{~ms}$ ) and ORDINATE defining the magnitude of the load (1177 N). The magnitude of the load was equivalent to the experimental cutting force reported in reference [56], but was reduced by 2000 times $(S F O=-2.0 E-03)$ to compensate for the limitations in mesh distortion of the Lagrangian formulation.

\subsubsection{Definition of Contact}

Since plane strain conditions were utilized in the model, the contact was two-dimensional and defined in *CONTACT_2D_AUTOMATIC_SURFACE_TO_SURFACE (pgs. 193, 196 and 199). The master contact entities and slave contact entities were defined by two part sets ( ${ }^{*}$ PART_SET_LIST on pgs. 193, 196 and 199) with the tool as the master and workpiece as the slave. A stiffness penalty factor of 10 was found to be sufficient in preventing penetration of the tool into the workpiece. This type of contact algorithm enables the user to define a coefficient of friction varying between the static and dynamic value as a function of relative velocity of the surfaces in contact as detailed in Section 2.2.3. Although the friction between the chip and the tool at the rake face does indeed vary between the sticking and sliding region (Section 2.1.3) due to the difficulty of characterizing this behaviour, the decay coefficient was set to a very high value $(D C=1000$ ) so that the coefficient of friction $\mu$ would always equal to the dynamic component. ( $\mathrm{FD}=0.6[67])$.

\subsubsection{Hourglass Control}

Given the high strain nature of the deformation problem at hand and the fact that plane strain elements contain only one integration point, hourglassing of elements must be considered. The hourglass effect, described in Section 2.2.7, was monitored by setting HGEN $=2$ in the ${ }^{*}$ CONTROL_ENERGY card (pgs. 191, 194 and 197), and controlled through the Flanagan-Belytschko stiffness method type 3 designated in ${ }^{*}$ CONTROL_HOURGLASS 
(pgs. 191, 194 and 197) as $I H Q=5$. This method of hourglass control is preferred for situations experiencing large deformation.

\subsubsection{Collected Output}

Several sets of data, specified by the *DATABASE_OPTION on pgs. 191, 194 and 197, were collected through the simulation. The information was stored in ASCII files at intervals of $1.0 \mu \mathrm{S}(\mathrm{DT})$. The GLSTAT and MATSUM files were generated to provide information on global statistics, including: energies, external work, velocities of parts and rigid bodies, and time step information. The translation and rotation displacements, velocities, and accelerations of nodes were tracked in the NODOUT file, and could be used in providing information on tool or chip velocity, tool displacement, and many other details on the dynamics of the problem. Particulars of the rigid tool displacement, velocity, and acceleration could also be obtained from the RBDOUT ASCII file. The RCFORC file, recorded the resultant forces between the rigid tool and the workpiece.

Plotting output states and data over the geometry of the model was enabled by

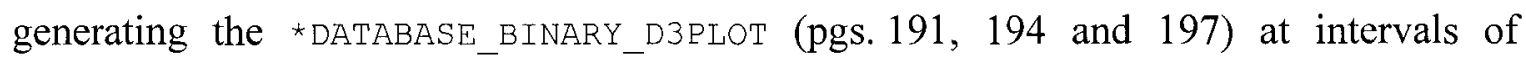
$25.0 \mu \mathrm{s}$ (DT/CYCL), while the *DATABASE_BINARY_D3THDT (pgs. 191, 194 and 197) file was generated at the same time intervals to record time history data for element subsets.

\subsubsection{Simulation Execution}

The simulation was set to terminate at $0.5 \mathrm{~ms}$, as designated by the ENDTIM parameter in the ${ }^{*}$ CONTROL_TERMINATION card (pgs. 191, 194 and 197). The time step scale factor (TSSEAC) was defined as 0.5 in the ${ }^{*}$ CONTROL_TIMESTEP card (pgs. 191, 194 and 197).

\subsection{MESH ADAPTIVITY}

To overcome the excessive element distortion arising from large material deformation and displacement in the tool tip area, adaptivity was applied to the mesh of the workpiece in latter segments of the simulation when the distortion was most severe. 
Through adaptivity, shell elements are subdivided into smaller elements when the original elements of the mesh lose accuracy due to mesh distortion, thereby lowering the potential of decreasing accuracy of the simulation with minimal increase in computational cost. The two methods utilized in applying adaptivity were: 1) adapting the mesh of the entire workpiece, and 2) adapting a fraction of the workpiece mesh, which surrounds the tool tip area or the area of most excessive distortion. The numerical set-up followed much of that in the Lagrangian mesh sensitivity study outlined in Section 4.1, including: element and material properties, boundary and loading conditions, contact definition, collected output, and simulation execution. Alterations to the geometry and additional executed keyword commands are described below.

\subsubsection{Geometry}

Because activating adaptivity is aimed towards eliminating excessive element distortion, the coarsest Lagrangian mesh was chosen for the simulation. Since the elements were plane strain elements, r-adaptivity was used; it refines the mesh of the entire designated part, as discussed in Section 2.2.5. The first approach to adapting the workpiece was to activate adaptivity for the entire part ( $P I D=2$ on pg. 194), which included the entire workpiece mesh. For the full workpiece adaptivity no adjustments were made to the mesh of the model, as shown in Figure 4.4 (a).

Secondly, since the literature review (Section 2.1.3) has shown that severe deformation is largely concentrated at the tool tip, the workpiece elements surrounding the tool tip were transferred to a new part ( $P I D=3$ on pg. 198); only this part was specified to be adapted in an effort to simplify and shorten the computation time for mesh adaptivity. The high deformation area surrounding the tool tip would be adapted while keeping the rest of the mesh coarse. The mesh of the partial adaptivity study is shown in Figure 4.4 (b), where the darker elements surrounding the tool tip are those placed in a separate part for adaptivity.

\subsubsection{Activating Adaptivity}

Adaptivity parameters were defined in the keyword *CONTROL_ADAPTIVE (pgs. 194 and 197), and adaptivity was set to occur in the second half of the simulation 
when most distortion takes place. For full mesh adaptivity, the first adaptation was to occur at $0.3 \mathrm{~ms}$ (TBIRTh) and repeat at intervals of $50.0 \mu \mathrm{s}$ (ADPFREQ) until the termination time of $0.5 \mathrm{~ms}$ (TDEATH). For partial mesh adaptivity, the first adaptation was to occur at $0.0 \mathrm{~s}$ (TBIRTH) and repeat at intervals of $50.0 \mu$ S (ADPFREQ) until the termination time of $0.5 \mathrm{~ms}$ (TDEATH). The method of adaptivity was two-dimensional r-adaptive remeshing ( $A D P T O L=8)$, and two-pass adaptivity was utilized as explained in Section 2.2.5. In this type of remeshing method, a completely new mesh is generated with a new element size specified in the card, which in this case was $30 \mu \mathrm{m}$ (ADPTOL) reducing the element side length to half of the original value (as specified in the input file) or quarter of the original area. Adaptivity is activated for a particular part, by setting the ADPOPT parameter in its corresponding *PART card to " 2 " standing for two-dimensional $r$-adaptive remeshing. In the full adaptivity study, remeshing was done for the entire workpiece ( $\mathrm{PID}=2$ on pg. 194), while for the partial adaptivity study it was done only for the newly formed part ( $\mathrm{BD}=3$ on pg. 198) containing elements adjacent to the tool tip. 


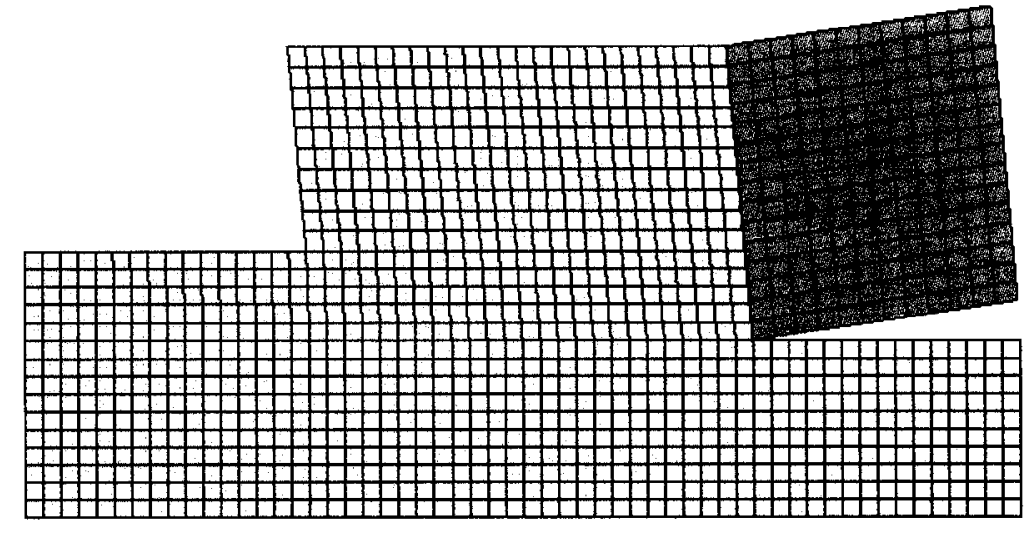

(a)

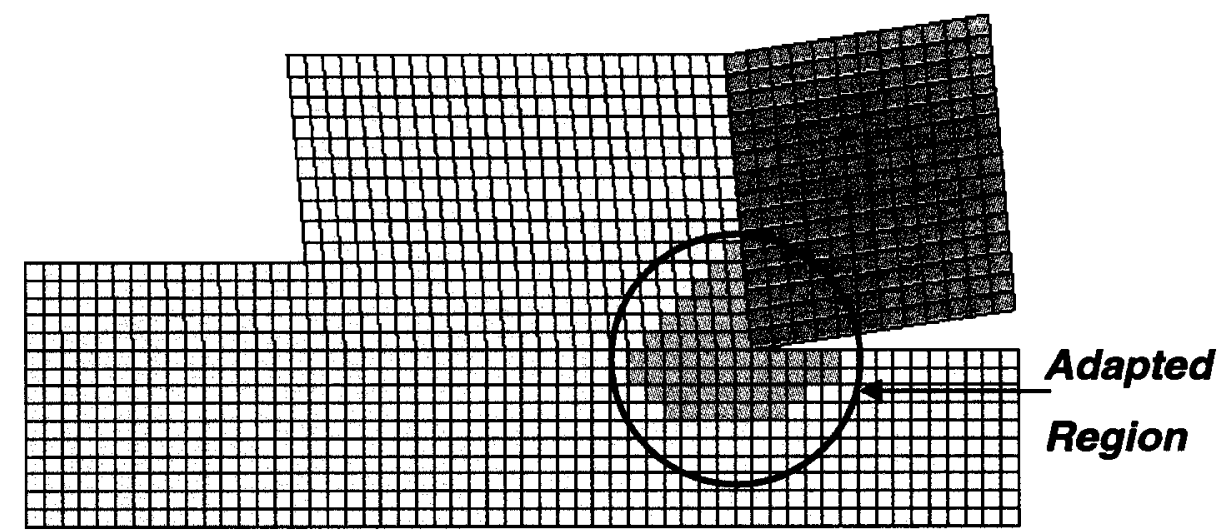

(b)

Figure 4.4: Mesh generated for the adaptivity study, including those for: (a) full workpiece adaptivity, and (b) partial workpiece adaptivity concentrated at the tool tip. 


\section{CHAPTER 5}

\section{Finite Element Procedures II : Metal Cutting Model Employing the ALE Element Formulation}

The ALE element formulation enables the smoothing of a mesh by remapping the coordinates of distorted elements into a new configuration, resulting in elements that have an acceptable level of distortion. The study of the ALE element formulation was conducted under incipient and steady state chip formation conditions. As with the Lagrangian procedures, presented in Chapter 4, the workpiece and tool were modeled with accordance to the experimental set-up of Elmadagli and Alpas [56]. The following cases were studied with the ALE element formulation:

- Steady State Chip Formation: ALE smoothing was activated in geometry similar to that of Mesh (b) in the Lagrangian study (Section 4.1.1), and compared with Lagrangian results.

- Incipient Chip Formation: Effect of using the ALE element formulation to model an undeformed workpiece subjected to orthogonal cutting was studied.

A summary of the input files compiled for the ALE study is enclosed in Appendices $\mathrm{F}$ and $\mathrm{G}$ for the steady state and incipient cases, respectively. The most pertinent parts of the input files are contained in the text below, and all other details can be found in LS-DYNA Keyword User's Manual: Volumes I and II [30, 32].

The results of the simulations performed with the ALE element formulation are presented in Chapter 8.

\subsection{STEADY STATE CHIP FORMATION}

\subsubsection{Geometry and Mesh Generation}

Currently an element capable of modeling plane strain conditions while incorporating ALE smoothing does not exist; as such plane strain conditions could not be applied in the ALE study, and the model was expanded to include a third dimension. In 
FEMB, Mesh (b) (Figure 4.2 (b)) from the Lagrangian study outlined in Chapter 4 was re-orientated into the xz-plane and extruded in the $y$-direction by $1500 \mu \mathrm{m}$ to form the 3-dimensional geometry shown in Figure 5.1. The tool was designated as part number 2 ( $P I D=2$ on pgs. 201 and 204), and the workpiece was designated as part number 1 ( $P I D=1$ on pgs. 201 and 204). The tool was modeled as a surface, which appeared as a solid line in the xz-plane. The clearance and rake angles were specified as 8 degrees and negative 5 degrees, respectively, consistently with experimental set-up [56]. The tool tip was given a radius of approximately $25 \mu \mathrm{m}$ to more precisely model the experimental tool geometry, which was not perfectly sharp. As with the Lagrangian element formulation,

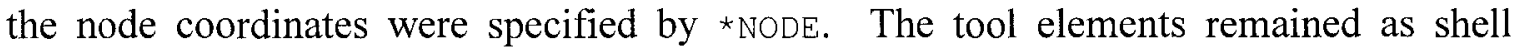
elements and were defined by *ELEMENT_SHELL (pgs. 201 and 204). The workpiece elements were now three-dimensional hexahedral elements, and the *ELEMENT_SOLID card (pgs. 201 and 204) was used to define the eight nodes of each of these elements. The hexahedral workpiece elements varied in size from a minimum of $30 \mu \mathrm{m} \times 30 \mu \mathrm{m} \times 60 \mu \mathrm{m}$ in the PDZ and SDZ, to a maximum of $60 \mu \mathrm{m} \times 60 \mu \mathrm{m} \times 60 \mu \mathrm{m}$ in the remainder of the mesh. The shell elements in the tool also varied in size from a minimum size of $20 \mu \mathrm{m} \times 25 \mu \mathrm{m}$ at the tool tip to a maximum size of $100 \mu \mathrm{m} \times 100 \mu \mathrm{m}$ at the rake face and clearance face extremities.

\subsubsection{Element Properties}

The workpiece elements used for the ALE study were ELFORM $=5$ specified in ${ }^{\star}$ SECTION_SOLID, which is an ALE element with one integration point. Although this card provides the option of specifying smoothing parameters, the parameters themselves were defined in the ${ }^{\star}$ CONTROL_ALE (pgs. 200 and 203) card, which enables the user to have more control over the smoothing. Elements of the tool were defined as Belytschko-Tsay type by setting ELFORM $=2$ in the ${ }^{\star}$ SECTION_SHELL card on pg. 201. The thickness of the tool shell elements was set to an arbitrary value of $1.0 \mu \mathrm{m}$. 


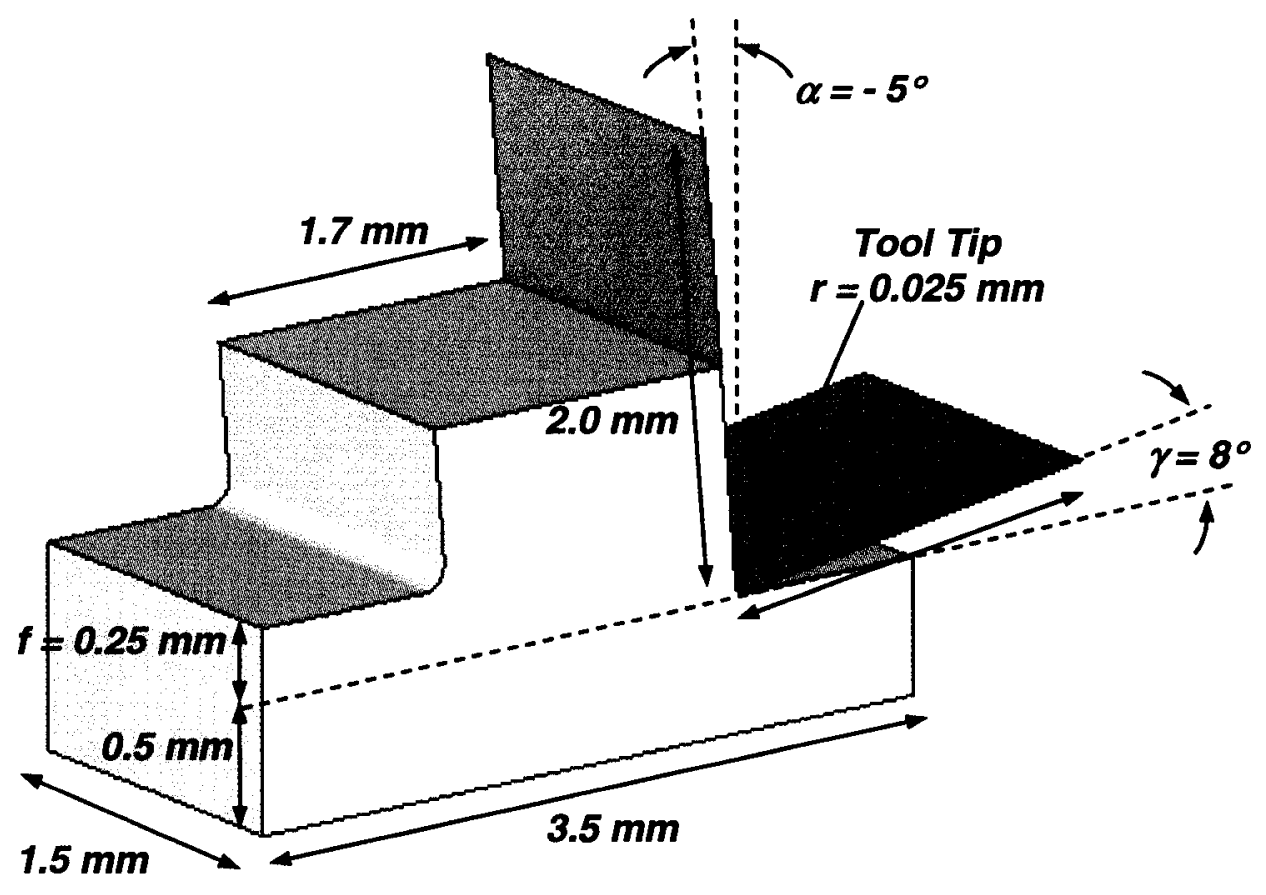

(a)

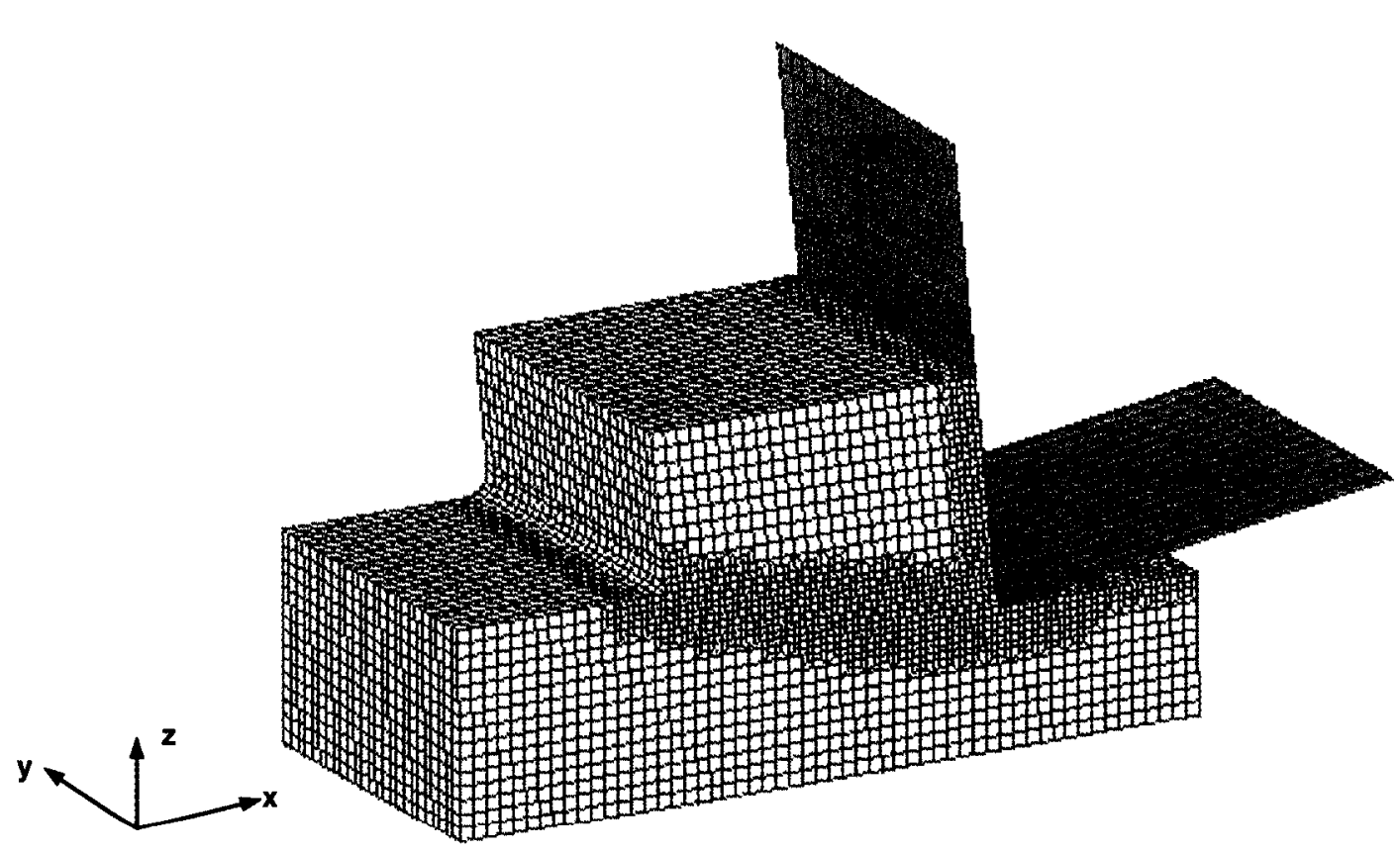

(b)

Figure 5.1: Geometry of workpiece and tool used in the ALE element formulation analysis of steady state chip formation showing: (a) relevant dimensions, and (b) generated mesh. 


\subsubsection{Material Properties}

Material properties used for the tool were identical to ones used in the Lagrangian studies described in Section 4.1.3. ALE smoothing is not compatible with the material model established in the Lagrangian study, which was Material type 24; so a new material model was developed for the workpiece in the ALE study.

To minimize computational time, the material model chosen to define the workpiece was *MAT_PLASTIC_KINEMATIC (pgs. 201 and 204), or Material type 3, which models isotropic and/or kinematic hardening. For this particular material model, the stress-strain curve was bilinear and required the definition of the elastic modulus and tangent modulus. The properties of commercial purity copper $(\mathrm{C} 11000)$ required in the definition of the material model included density, Young's modulus, Poisson's ratio, and yield strength, which appear in Table 2.1. The plastic region of the stress-strain curve formed by setting $E_{t}=15 \mathrm{GPa}$ is presented as a solid line in Figure 5.2, along with the stress-strain curve produced in reference [56] fitting the Voce equation, which is shown as a dashed line. Isotropic hardening was activated by setting BETA=1 (pgs. 201 and 204) in the input file; strain rate effects, although can be applied, were neglected to simplify the analysis.

Details of the tool material model are described in section 4.1.3, and properties required for its definition are listed in Table 2.2.

\subsubsection{Applied Boundary Conditions and Motion}

For the ALE study, the tool was prescribed motion while the workpiece was kept stationary. In order to keep the workpiece stationary all nodes on the bottom of the workpiece were restricted in all DOF. Nodes on the workpiece surfaces parallel to the $\mathrm{xz}$-plane were assigned a plane of xz-symmetry (restricting translation in the $\mathrm{y}$-direction and rotation about the $\mathrm{x}$-axis and z-axis) to model an infinite depth in the y-direction, resembling plane strain conditions. The nodal constraints were defined using *BOUNDARY_SPC_NODE, described earlier in Section 4.1.4. 


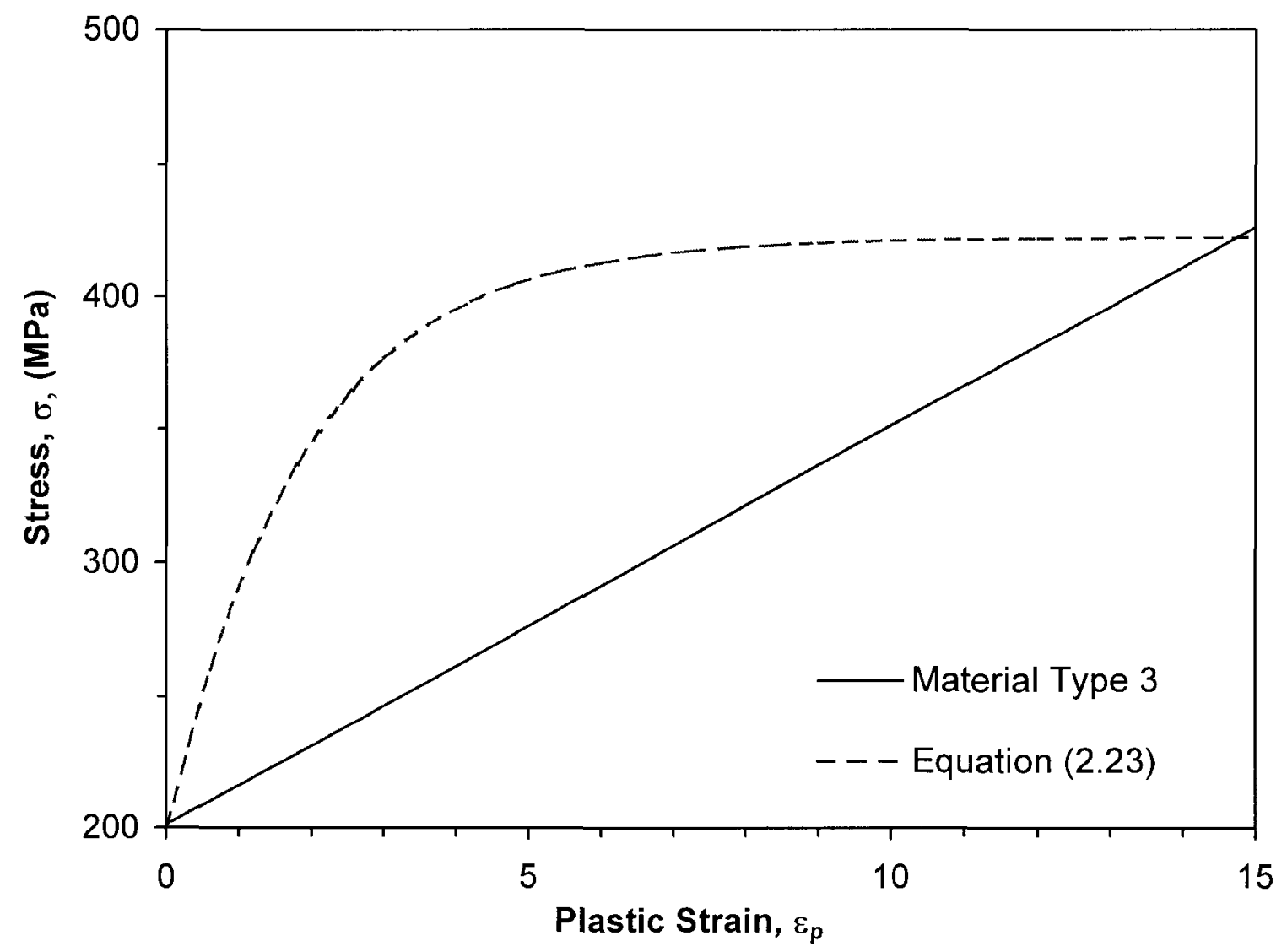

Figure 5.2: Comparison of the plastic region of the linear stress-strain approximation of Material type 3, to that of the Voce equation [56]. 
Since the tool of the ALE study was modeled with shell elements rather than solid elements like in the Lagrangian study, the tool motion was no longer generated by forces in ${ }^{*} \angle O A D_{-} R I G I D \_B O D Y$ card but rather by velocities in the *BOUNDARY_PRESCRIBED_MOTION_RIGID card on pgs. 202 and 205. The definition of velocity was depicted by setting $V A D=0$ and it was given an $x$-translation $D O F(D O F=1)$ in the negative direction $(S F=-1.0)$. The magnitude of the velocity was defined in *DEFINE_CURVE, which was referenced as load curve $L C I D=1.0$, with ABCISSA values as time in seconds and ORDINATE values as velocity in $\mu \mathrm{m} \cdot \mathrm{s}^{-1}$. By scaling the values of the ordinate parameter $(\mathrm{SFO}=6 . \mathrm{OE}+05)$, a constant velocity of $600 \mathrm{~mm} \cdot \mathrm{s}^{-1}$ was applied to reflect the experimental set-up [56].

\subsubsection{Definition of Contact}

The contact between the three-dimensional models of the tool and workpiece were defined in ${ }^{*}$ CONTACT_SURFACE_TO_SURFACE (pgs. 202 and 205). The master and slave entities were both designated as parts (SSTYP=MSTYP=3), where the tool was the master part (MSID=2) and the workpiece was the slave part (SSID=1). A stiffness penalty factor of 20 was applied to prevent penetration of the tool into the workpiece. The defined friction parameters were identical to the values specified in Section 4.1.5. for *CONTACT_2D_AUTOMATIC_SURFACE_TO_SURFACE. The value of the coefficient of friction $(\mu)$ was defined as constant by setting $\mathrm{DC}=1000$, and was given a value of 0.6 .

\subsubsection{Hourglass Control}

The ALE elements used to model the workpiece contained one integration point; therefore there was a potential for hourglassing. The hourglass effect, outlined in Section 2.2.7, was monitored by same methods as those outlined in Section 4.1.6. 


\subsubsection{Activating ALE Smoothing}

ALE formulation, previously described in Section 2.2.2, was activated through the ${ }^{\star}$ CONTROL_ALE card on pgs. 200 and 203, by setting DCT=3. Advection was performed through a second-order Van Leer [28, 29] method $(\mathrm{METH}=2)$, which allows the interpolation of properties within an element into a piecewise function. ALE smoothing was based on simple average, volume and equipotential weight factors, whose contribution to the total smoothing calculation was $0.1 / 4.1,2 / 4.1$ and $2 / 4.1$, respectively, as determined by several trial simulations. The smoothing weight factors are outlined in more detail in the LS-DYNA Theoretical Manual [27]. Mesh smoothing was set to occur after every cycle $(\mathrm{NADV}=1)$ throughout the entire simulation starting from a time of 0.0 seconds (START) and ending at a time of 0.005 seconds (END).

\subsubsection{Collected Output}

Data to be collected throughout the simulation were specified by the *DATABASE_OPTION command on pgs. 200 and 203. The collected information was recorded in ASCII files at intervals of $1.0 \mu \mathrm{S}$ (DT). In addition to GLSTAT, MATSUM, RCFORC and RBDOUT files (defined in Section 4.1.8), an SPCFORC file was also generated during the ALE smoothing simulations. This file records the $\mathrm{x}, \mathrm{y}$ and $\mathrm{z}$ forces and moments occurring between the two parts defined in the contact card, or in this case the tool and workpiece.

As in the Lagrangian study, *DATABASE_BINARY_D3PLOT was used to plot output states and data distribution at intervals of $25.0 \mu \mathrm{s}$ (DT/CYCL). History data for element subsets was generated through *DATABASE_BINARY_D3THDT at the same intervals of time.

\subsubsection{Simulation Execution}

The simulation was terminated at $0.5 \mathrm{~ms}$, as specified by ENDTIM in the *CONTROL_TERMINATION card on pgs. 200 and 203. The designated time step scale factor (TSSFAC) was 0.5, as was defined by the ${ }^{\star}$ CONTROL_TIMESTEP card on pgs. 200 and 203. 


\subsection{INCIPIENT CHIP FORMATION}

The numerical set-up for the study of ALE element formulation during incipient chip formation was very similar to that of the steady state chip formation. The input for the two cases differed only in geometry, element properties, and boundary conditions; all other definitions are consistent with those outlined in Section 5.1, including: material properties, applying tool motion, contact definition, collected output, and simulation execution.

\subsubsection{Geometry}

The geometry of the workpiece and the tool was altered in an effort to study the machining process from its initiation, beginning with an undeformed workpiece and an approaching tool. The modeling was completed in FEMB and the geometry of each generated part is shown in Figure 5.3 (a). Mesh distribution was kept uniform through the entire geometry, as is seen in Figure 5.3 (b), which shows the mesh in the xz-plane.

The model consisted of two parts: 1) the workpiece ( $P I D=1$ on pg. 204) made up of 1800 hexahedral solid elements with size $100 \mu \mathrm{m} \times 100 \mu \mathrm{m} \times 111 \mu \mathrm{m}$, and 2) the tool ( $P I D=2$ on pg. 204) made up of 1000 hexahedral solid elements with elements of size $90 \mu \mathrm{m} \times 100 \mu \mathrm{m} \times 100 \mu \mathrm{m}$. The most pertinent dimensions were modeled according to the experimental set-up [56]; the remainder of the dimensions were chosen arbitrarily.

\subsubsection{Element Properties}

The workpiece elements used for this study were ELFORM=5 specified in the *SECTION_SOLID card on pg. 204, identical to those described in Section 5.1.2. The tool geometry was converted to solid hexahedral elements therefore they were also characterized by the ${ }^{*}$ SECTION_SOLID card on pg. 204. Since the tool was rigid and did not undergo any deformation, ALE elements were not used for this part, but rather the default constant stress solid elements were used ( $E L F O R M=1)$. 


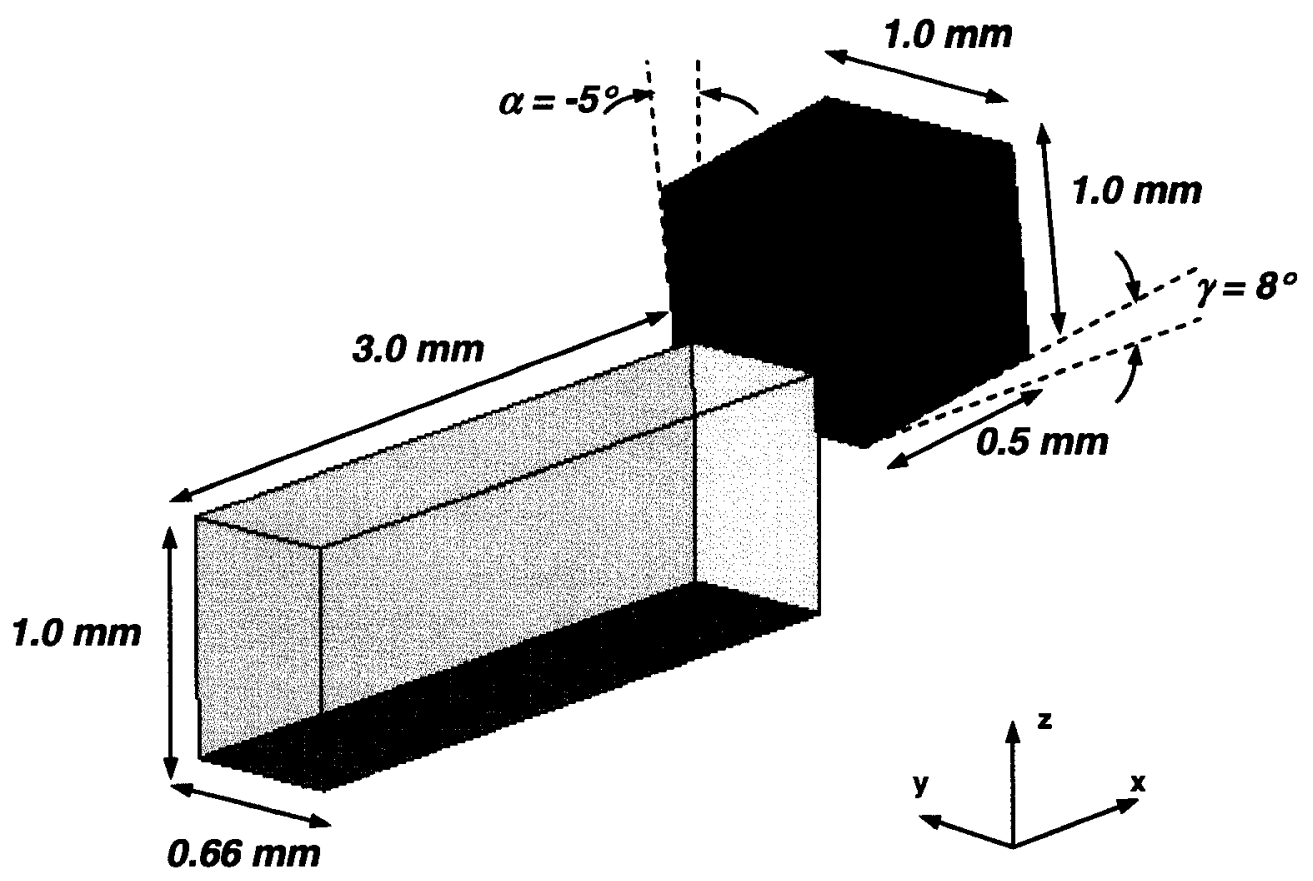

(a)
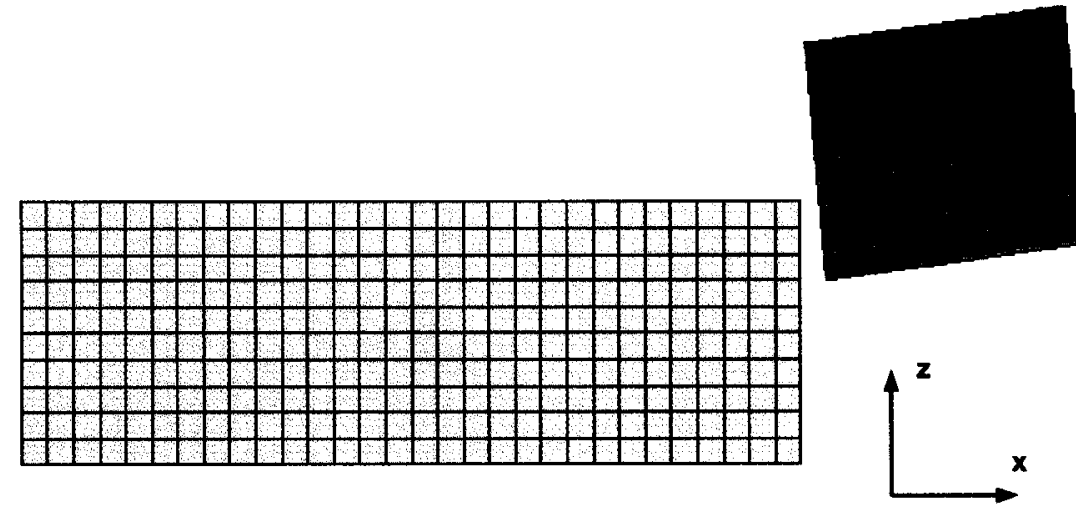

(b)

Figure 5.3: Geometry of workpiece and tool used in the ALE element formulation analysis of incipient chip formation showing: (a) relevant dimensions, and (b) generated mesh. 


\subsubsection{Applied Boundary Conditions}

As with the first ALE study, the tool was prescribed motion while the workpiece was kept stationary. To prevent the workpiece from moving all nodes at the bottom surface of the tool were constrained in all DOF. Sides of the workpiece parallel to the xz-plane, where defined as planes of xz-symmetry (restricting translation in the $\mathrm{y}$-direction and rotation about the $\mathrm{x}$-axis and $\mathrm{z}$-axis) to create an infinite workpiece length in the $y$-direction and replicate a plane strain scenario. None of the nodes in the tool were constrained, and its motion was applied in the same manner as described in Section 5.1.4. 


\section{CHAPTER 6}

\section{Finite Element Procedures III :}

\section{Metal Cutting Model Employing the Eulerian Element Formulation}

The application of the Eulerian element formulation for modeling of metal cutting was conducted on incipient chip formation. The chip and tool were modeled according to the experimental work of Elmadagli and Alpas [56]. The critical components of the input file are described below and others are detailed in the LS-DYNA Keyword User's Manual: Volumes I and II [30, 32].

In summary, the following simulations were performed using the Eulerian element formulation:

- Workpiece Moving into a Stationary Tool:

- Model 1: Application of the Johnson-Cook material model for the workpiece, with parameters derived from experimental stress-strain values calculated in [56].

- Model 2: Application of the Johnson-Cook material model for the workpiece, with parameters obtained from Johnson and Cook [68].

- Model 3: Application of the hydrodynamic material model for the workpiece, with stress-strain behaviour curve-fitted to the Voce equation flow curve produced in [56]

The input corresponding to Material Models 1, 2 and 3, are presented in Appendices H, I, and J, respectively.

The results of the simulations performed with the Eulerian element formulation are presented in Chapter 9.

\subsection{WORKPIECE MOVING INTO A STATIONARY TOOL}

\subsubsection{Geometry and Mesh Generation}

The model used in the study of the Eulerian element formulation, consisted of three parts namely, the workpiece ( $P I D=1$ on pgs. 206, 209 and 212), the airmesh ( $P I D=2$ 
on pgs. 206, 209 and 212), and the tool (PID=3 on pgs. 207, 210 and 213). Since the airmesh $(P I D=2)$ did not initially contain any material, its material was defined as void using the *INITIAI_VOID_PART cards on pgs. 207,210 and 213.

A three-dimensional view of the set-up is shown in Figure 6.1 (a). The first geometry generated included one plane of symmetry, which ran along the xy-plane and cut the model in half, as shown in Figure 6.1 (b). A summary of the number of elements in each of the three parts modeled is shown in Table 6.1. The experimental wall thickness-to-feed ratio was greater than 10 and reflects a plane strain condition, which was utilized in the second geometry where three elements were modeled through the thickness (in the z-direction), with two planes of symmetry on each of the xy faces to simulate infinite depth. The second geometry, with the two planes of symmetry, is shown in Figure 6.1 (c); the number of elements constructing each of the parts in this model is summarized in Table 6.1. Utilizing two planes of symmetry to model plane strain conditions reduced the number of elements of each part by one order of magnitude. Although each model depth and the number of elements varied considerably, the element size in the cross section was constant for both models, and is shown in Figure 6.2. The mesh size was refined at the tool tip level, to improve accuracy of calculations in this high deformation area. The feed $(0.25 \mathrm{~mm})$, clearance angle ( 8 degrees), and rake angle (negative 5 degrees) were modeled in consistency with the experimental set-up, and the remainder of the dimensions were chosen arbitrarily.

\begin{tabular}{|c|c|c|c|c|}
\hline Part & $\begin{array}{c}\text { Number of Elements } \\
\text { in model with one } \\
\text { plane of symmetry }\end{array}$ & $\begin{array}{c}\text { Number of Elements } \\
\text { in model with two } \\
\text { planes of symmetry }\end{array}$ & $\begin{array}{c}\text { Minimum } \\
\text { Element } \\
\text { Size }(\boldsymbol{\mu} \mathbf{m})\end{array}$ & $\begin{array}{c}\text { Maximum } \\
\text { Element Size } \\
(\mu \mathrm{m})\end{array}$ \\
\hline Tool & 6336 & 660 & $38 \times 37$ & $60 \times 75$ \\
\hline Workpiece & 676 & 78 & $92 \times 54 \times 276$ & $92 \times 120 \times 276$ \\
\hline Airmesh & 181424 & 16008 & $92 \times 54 \times 276$ & $96 \times 120 \times 276$ \\
\hline
\end{tabular}

Table 6.1: Number of elements in each of the two geometries of the Eulerian element formulation study. 


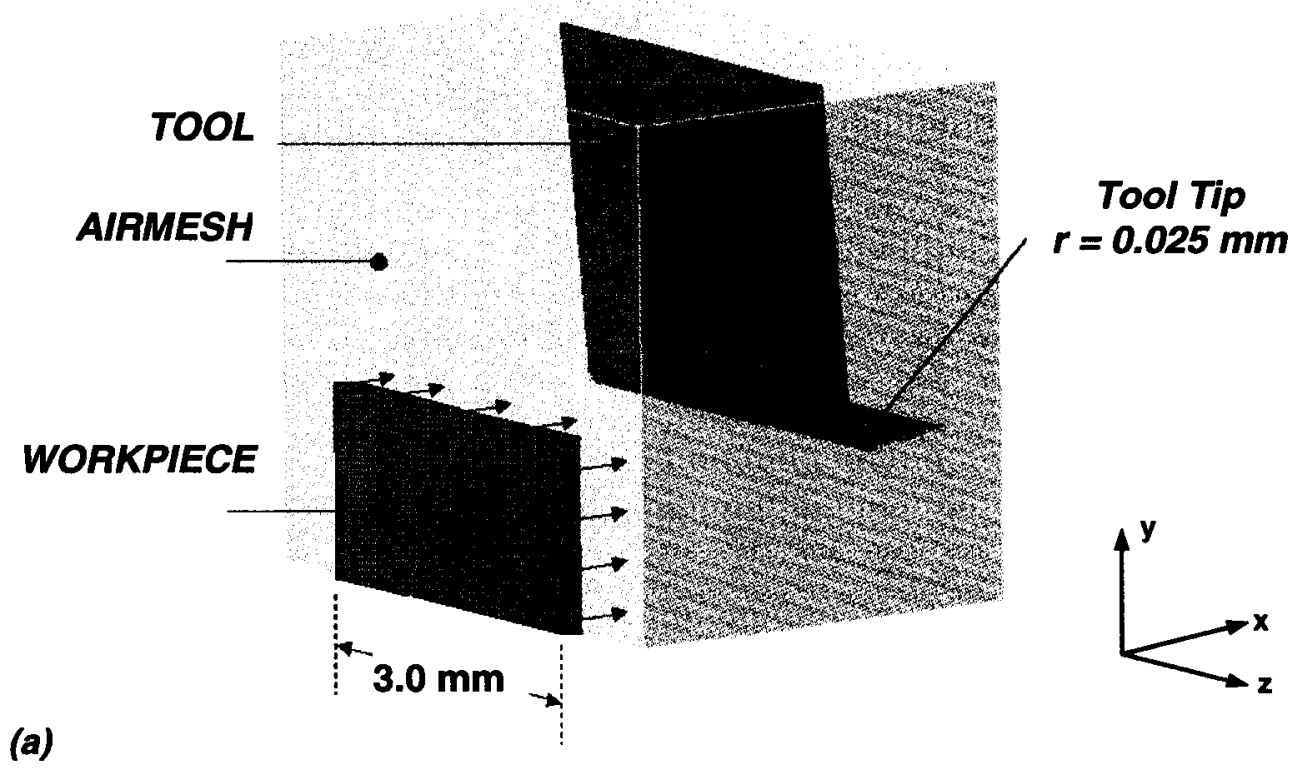

(a)

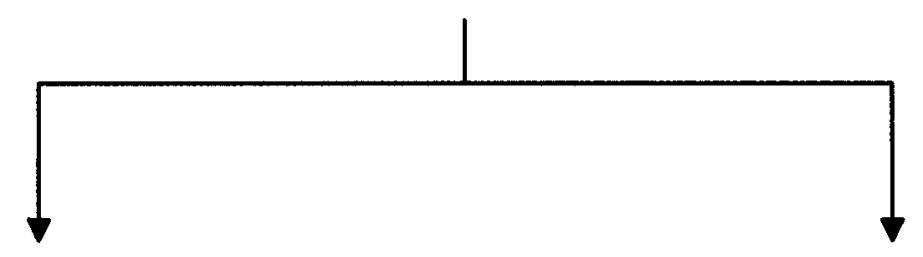

\section{ONE PLANE OF SYMMETRY}

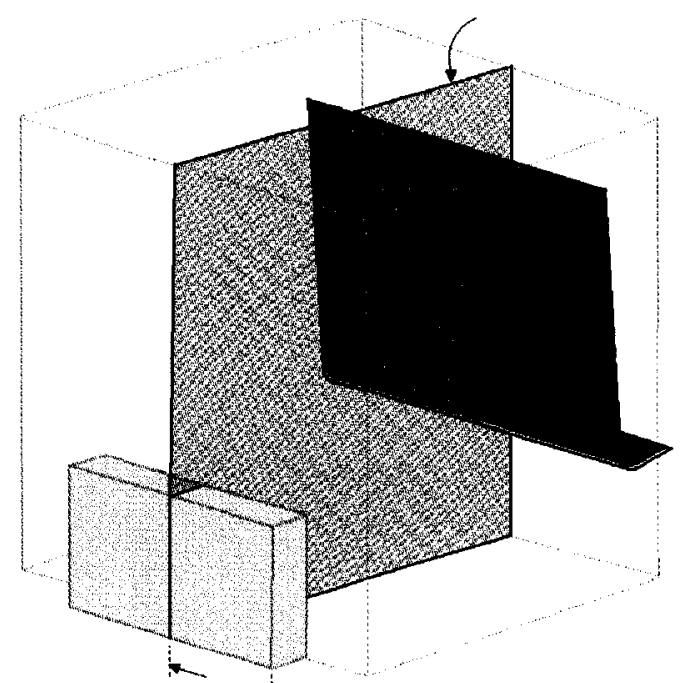

(b)
TWO PLANES OF SYMMETRY

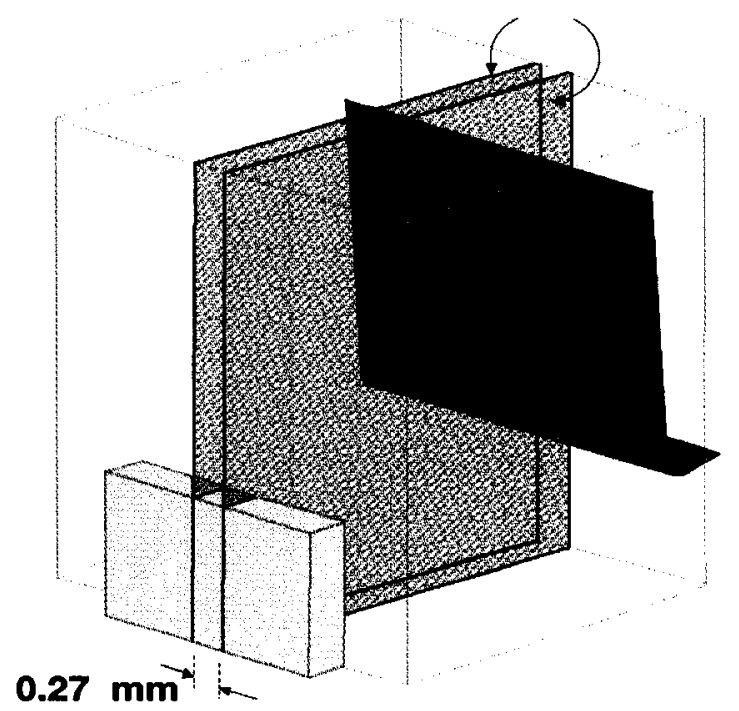

Figure 6.1: Geometry of the Eulerian FE model including: (a) three-dimensional view showing all pertinent parts and workpiece depth in millimetres, (b) geometry of workpiece modeled with one plane of symmetry reducing the workpiece depth by $50 \%$, and (c) geometry of workpiece modeled with two planes of symmetry reducing workpiece depth to $9 \%$ of original depth labelled in (a). 


\section{Plane parallel to $x y$-axis}

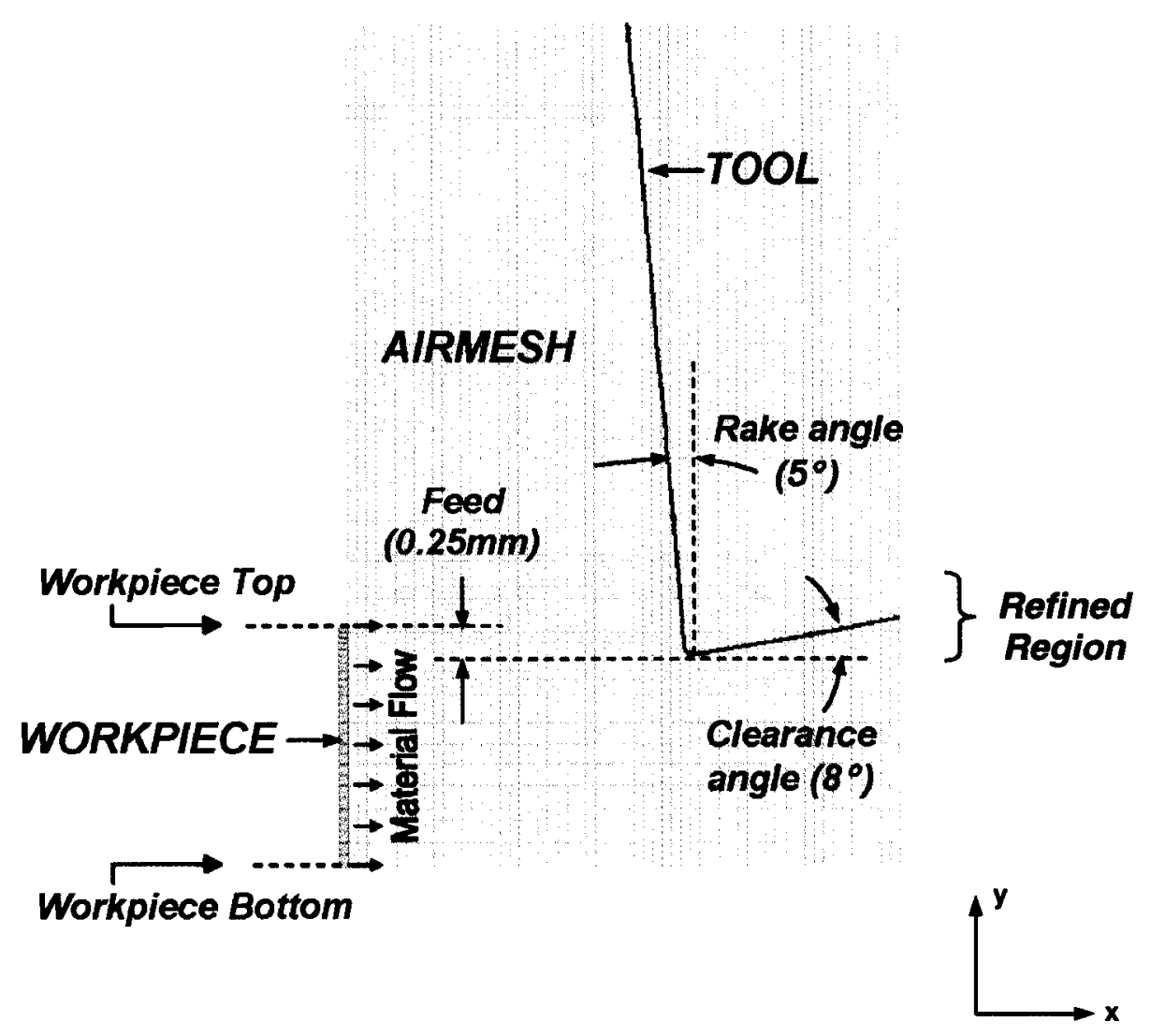

Figure 6.2: Cross-sectional view of the Eulerian geometry, showing the element distribution and relevant dimensions. 
Both the workpiece and the airmesh were modeled as hexahedral elements, whose nodes were defined in ${ }^{*} \operatorname{NODE}$ (pgs. 208, 211 and 214); the eight nodes defining the

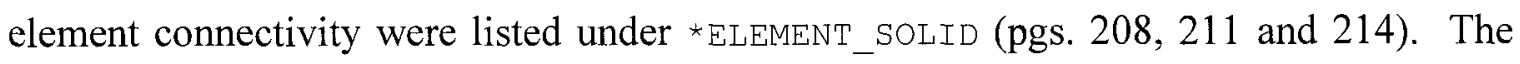
tool was constructed of shell element, whose nodes were also defined in *NODE; however, element connectivity was specified using *ELEMENT__SHELL (pgs. 208, 211 and 214).

\subsubsection{Element Properties}

The hexahedral workpiece and airmesh elements were of a single-point integration solid Eulerian type with single material and void, as was defined by setting ELFORM $=12$ in their respective ${ }^{*}$ SECTION_SOLID cards on pgs. 207, 210 and 213. In addition, the workpiece elements were also assigned a pressure inflow, which provided a constant inflow of the material through the workpiece elements and into the airmesh at a designated velocity. The tool elements were designated as Belytschko-Tsay elements of a Lagrangian formulation by indicating ELFORM $=2$ in the ${ }^{*}$ SECTION_SHELL cards on pgs. 208, 211 and 214. The thickness of the tool shell elements was arbitrarily chosen to be $0.1 \mathrm{~mm}$ at each of the four nodal points of an element (T1, T2, T3 and $\mathrm{T} 4)$.

\subsubsection{Material Properties}

The material chosen for modeling of the Lagrangian tool was Material type 20

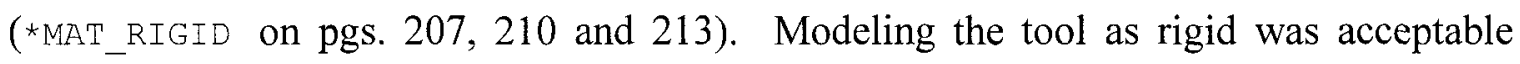
because tool properties and its behaviour were not studied in the scope of the project. It was also beneficial to use this material model due to the simplicity of computations within the rigid material, which shortened simulation time. The properties of the tool were kept consistent with the experimental set-up and have previously been listed in Table 2.2.

Two different material types were investigated for the workpiece: (a) the Johnson-Cook constitutive equation incorporating strain, strain rate, and temperature effects, and (b) elastic plastic hydrodynamic material defining a flow curve fitted to the experimental data. Details of each developed material model are following, with a description of the equation of state required by both material types. 


\section{Johnson-Cook Constitutive Equation}

The Johnson-Cook material model (*MAT_JOHNSON_COOK pgs. 207 and 210) is referenced as Material type 15 in LS-DYNA and is often used in numerical modeling due to its ability to accurately predict the behaviour of materials subjected to a broad range of strains, strain rates, and temperatures [68]. In this study, the effectiveness of the Johnson-Cook constitutive model, in predicting behaviour of a material subjected to orthogonal cutting, was investigated.

The general form of the Johnson-Cook equation is as follows:

$$
\sigma=\left(A+B \bar{\varepsilon}^{p^{n}}\right)\left(1+C \ln \dot{\varepsilon}^{*}\right)\left(1-T^{* m}\right)
$$

where $A, B, n$ and $c$ and $m$ are material constants, $\bar{\varepsilon}^{p}$ is the equivalent plastic strain, $\dot{\varepsilon}^{*}$ is the normalized equivalent plastic strain rate, and $T^{*}$ is the homologous temperature defined by Equation (6.2),

$$
T^{*}=\frac{T-T_{\text {room }}}{T_{\text {melt }}-T_{\text {room }}}
$$

where $T_{\text {room }}$ is the specified room temperature, $T_{\text {melt }}$ is the melting temperature, and $T$ is the temperature of the material.

Johnson-Cook parameters $A, B, n, C$, and $m$, are typically derived from a series of dynamic tests, such as Taylor bar or torsion tests, performed at a wide range of temperatures and strain rates. In the presented work the Johnson-Cook parameters were obtained in two alternative ways. These methods were: 1) curve fitting the strain dependent terms of the Johnson-Cook equation into the experimental Voce equation, and 2) using parameters derived for OFHC copper, whose properties are very similar to the properties of ETP copper used in the experiments.

In the first version of the Johnson-Cook material model (herein referred to as Material Model 1), the relationship between stress and strain (stress is dependent only on the first term of the constitutive equation) was fitted to the experimental stress-strain flow curve defined through the Voce equation [56]. In the first term of Equation (6.1), the constant $A$ (also defined as the yield strength) was determined by extrapolating experimental data from Figure 2.20 at zero plastic strain. Constants $B$ and $n$, the work hardening exponent, were found by curve fitting a logarithmic plot of the term 
$\left(A+B \bar{\varepsilon}^{p^{n}}\right)$ to experimental stress/strain values shown in Figure 2.20. The remainder of the constants $(C$ and $m$ ) were obtained from Johnson and Cook [68]. The full extrapolation of parameters $A, B$ and $n$, is outlined in Appendix $\mathrm{K}$.

In the second Johnson-Cook material model (referred to as Material Model 2), the parameters were obtained from research of Johnson and Cook [68]. In actuality the parameters were specified as those for commercial purity OFHC copper (C10200) but were used to model the commercial purity ETP copper $(\mathrm{C} 11000)$ in the numerical simulations due to the similarity in properties between the two metals shown in Table 6.2.

\begin{tabular}{|l|c|c|}
\hline Properties & OFHC copper & ETP copper \\
\hline Composition & $99.95 \% \mathrm{Cu}+\mathrm{Ag}$ & $99.9 \% \mathrm{Cu}+0.04 \mathrm{O}$ \\
\hline$\rho\left(\mathrm{gm} \cdot \mathrm{cm}^{-3}\right)$ & 8.90 & $8.89-8.94$ \\
\hline $\mathrm{G}(\mathrm{GPa})$ & 44 & 47 \\
\hline $\mathrm{E}(\mathrm{MPa})$ & $117-132$ & $115-130$ \\
\hline Poisson's Ratio & 0.31 & 0.33 \\
\hline $\mathrm{T}_{\text {melt }}\left({ }^{\circ} \mathrm{C}\right)$ & 1083 & 1065 \\
\hline $\mathrm{C}_{\mathrm{p}}\left(\mathrm{J} \cdot \mathrm{kg}^{-1} \cdot{ }^{-0} \mathrm{C}^{-1}\right)$ & 385.15 & 384.7 \\
\hline
\end{tabular}

Table 6.2: Comparison between properties of commercial purity OFHC and ETP coppers obtained from ASM Metals Handbook: Volume 2 [58].

The Johnson Cook parameters for Material Model 1 and Material Model 2 are listed in Table 6.3. In addition, density, shear modulus, Young's modulus, and Poisson's ratio, already listed in Table 2.1, were also used.

\begin{tabular}{|c|c|c|}
\hline Properties and units & $\begin{array}{c}\text { Johnson-Cook } \\
\text { Model 1 (pg. 207) }\end{array}$ & $\begin{array}{c}\text { Johnson-Cook } \\
\text { Model 2 (pg. 210) }\end{array}$ \\
\hline$A(\mathrm{MPa})$ & 199.3 & $92.0[68]$ \\
\hline$B(\mathrm{MPa})$ & 105.2 & $292.0[68]$ \\
\hline$n$ & 0.325 & $0.310[68]$ \\
\hline$C[68]$ & 0.025 & 0.025 \\
\hline$m[68]$ & 1.09 & 1.09 \\
\hline
\end{tabular}

Table 6.3: The Johnson-Cook parameters used in Material Models 1 and 2. 


\section{Hydrodynamic Material Model}

The hydrodynamic material (*MAT_ELASTIC_PLASTIC_HYDRO on pg. 213) is referred to as Material type 10 within LS-DYNA. This material type models an elastic plastic hydrodynamic material and defines the stress-strain relationship through sixteen points fitted to the flow curve. The sixteen stress-plastic strain pairs used in the material model of the commercial purity copper (herein referred to as Material Model 3) are listed in Table 6.4 and were obtained from the Voce equation [56]. Other properties required to complete the model are density and shear modulus, both previously defined in Table 2.1.

\begin{tabular}{|c|c|c|c|}
\hline$\varepsilon_{p}\left(\mathbf{m m} \cdot \mathbf{m m}^{-1}\right)$ & $\sigma(\mathbf{M P a})$ & $\varepsilon_{p}\left(\mathbf{m m} \cdot \mathbf{m m}^{-1}\right)$ & $\sigma(\mathbf{M P a})$ \\
\hline 0.0 & 199.30 & 4.0 & 395.4 \\
\hline 0.5 & 251.1 & 5.0 & 406.4 \\
\hline 1.0 & 290.9 & 6.0 & 412.9 \\
\hline 1.5 & 321.4 & 7.0 & 416.7 \\
\hline 2.0 & 344.8 & 8.0 & 419.0 \\
\hline 2.5 & 362.8 & 10.0 & 421.1 \\
\hline 3.0 & 376.6 & 15.0 & 422.1 \\
\hline 3.5 & 387.2 & 20.0 & 422.2 \\
\hline
\end{tabular}

Table 6.4: Sixteen stress-plastic strain pairs derived from experimental results, and used to define the flow curve for commercial purity ETP copper.

The flow curves generated by the two Johnson-Cook Models as well as the curve generated by the hydrodynamic material model are shown in Figure 6.3. The flow curve of Material Model 3 (or the hydrodynamic material model) is represented by the experimental curve from which it was derived. The Material Model 1 flow curve is graphed using the first term of the Johnson-Cook equation, whose parameters were curve-fitted to experimental findings. The flow curve of Material Model 2 was obtained from Reference [68], and reflects data obtained from torsion tests.

\section{Equation of State}

As described in reference [31], when the amplitude of stress waves in a material exceeds the dynamic flow strength, the hydrostatic component of stress dominates and 


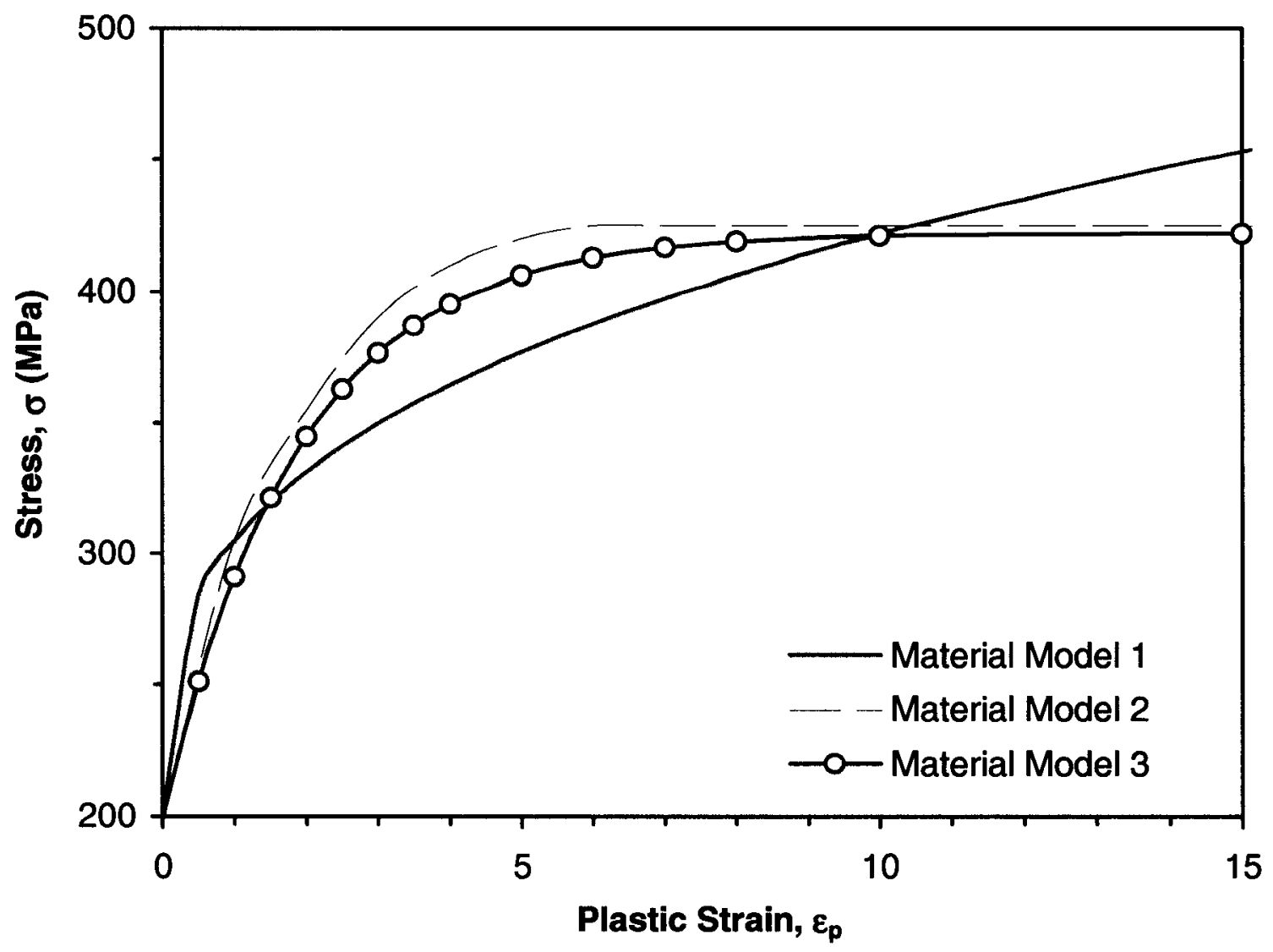

Figure 6.3: Comparison of flow curve obtained from Material Model 1, Material Model 2 and Material Model 3. 
the solid has no resistance to shear. The hydrostatic component of stress generates a high-pressure state, which travels through the material in the same manner as the stress wave causing a disturbance front to form. The disturbance front accumulates as it travels through the material because higher amplitude regions travel faster then lower amplitude regions. This accumulation of the disturbance front leads to a shock wave, which is defined as a discontinuity in pressure, temperature, and density.

The Grüneisen equation of state (EOS) [27, 31] was utilized to describe the pressure-volume relationship of the copper specimen for both the Johnson-Cook and hydrodynamic material models. This particular type of EOS relates the material shock velocity and particle velocity through a cubic equation and defines the pressure of a compressed material as:

$$
P=\frac{\rho_{o} C_{o}^{2} \xi\left[1+\left(1-\frac{\gamma_{o}}{2}\right) \xi-\frac{b}{2} \xi^{2}\right]}{\left[\left(1-\left(S_{1}-1\right) \xi-S_{2} \frac{\xi^{2}}{\xi+1}-S_{3} \frac{\xi^{3}}{(\xi+1)^{2}}\right]^{2}\right.}+\left(\gamma_{o}+b \xi\right) E_{v}
$$

where $\rho_{o}$ is the initial density, $E_{v}$ is the energy per initial volume, $C_{o}$ is the bulk sound speed, $S_{1}, S_{2}$, and $S_{3}$ are the linear, quadratic, and cubic coefficients, respectively, relating material shock velocity to particle velocity, $b$ is the coefficient of volume dependence of gamma, $\gamma_{o}$ is the initial value of the Grüneisen gamma, and $\xi$ is the compression defined as:

$$
\xi=\frac{\rho}{\rho_{0}}-1
$$

where $\rho$ is the density of the material. The ${ }^{\star}$ EOS_GRUNEISEN card (pgs. 207, 210 and 213) requires the definition of $C_{o}, S_{1}, S_{2}, S_{3}, \gamma_{0}$ and $b$, parameters obtained from work by Steinberg [69] and listed in Table 6.5. While the Johnson-Cook constitutive equation is used to determine the deviatoric component of stress, the EOS accounts for the hydrostatic component of stress; together they constitute the complete stress state. 


\begin{tabular}{|c|c|}
\hline Parameter & Value with units \\
\hline$C_{o}$ & $0.394 \mathrm{~cm} / \mu \mathrm{s}$ \\
\hline$S_{l}$ & 1.489 \\
\hline$S_{2}$ & 0 \\
\hline$S_{3}$ & 0 \\
\hline$\gamma_{0}$ & 2.02 \\
\hline$b$ & 0.47 \\
\hline
\end{tabular}

Table 6.5: Parameters necessary to complete the EOS required by the material models of the commercial purity copper workpiece, obtained from the work of Steinberg [69].

\subsubsection{Applied Boundary Conditions and Workpiece Motion}

To simulate the experimental set-up, the tool was kept stationary and a velocity was applied to the workpiece. The tool was restricted from motion by setting constraints on all of its nodes in all DOF using * BOUNDARY_SPC_NODE (pgs. 208, 211 and 214). To ensure that the flow of material throughout the simulation is horizontal despite deformation due to interaction with the tool, the SPC method was also used to restrict material within the "workpiece" and along the "workpiece bottom", to travel in the $\mathrm{x}$-direction. Velocity of the material initially contained in the workpiece part was defined in the *BOUNDARY_PRESCRIBED_MOTION_OPTION cards on pgs. 208, 211 and 214. The

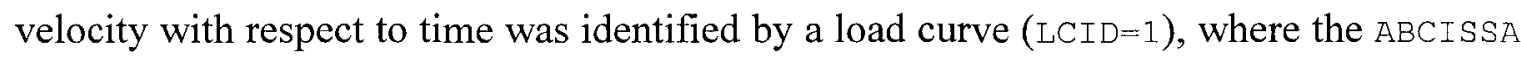
values were time varying from $0.0 \mathrm{~s}$ to $0.04 \mathrm{~s}$, and ORDINATE values were the velocity remaining constant at $600 \mathrm{~mm} \cdot \mathrm{s}^{-1}$. The velocity direction was specified as the $\mathrm{x}$-direction $(\mathrm{DOF}=1)$ and it was applied to nodes listed in *SET_NODE_LIST (pgs. 208, 211 and 214) referenced to as NSID $=50$, which included all nodes of the workpiece part. Although the material in the workpiece was designated to travel into the airmesh, the pressure inflow assigned to the workpiece elements ensured constant replenishment of the material into these elements. The planes of symmetry used in the geometries shown in Figure 6.1 (b) and (c), were activated by placing SPC's on all nodes lying on the plane, disabling motion in the z-direction and rotation about the $\mathrm{x}$-axis and $\mathrm{y}$-axis ( $\mathrm{DOFZ}=1$, DOFRX $=1$ and DOFRY=1), while allowing translation along the $\mathrm{x}$ and $\mathrm{y}$-directions as well as rotation about the $\mathrm{z}$-axis $(\mathrm{DOFX}=0, \mathrm{DOFY}=0$ and $\mathrm{DOFRZ}=0)$. 


\subsubsection{Definition of Contact}

The ${ }^{*}$ CONSTRAined_LAGRANGE_IN_SOLID (pgs. 207, 210 and 213) penalty-type (CTYPE $=4$ ) contact algorithm, was used to model the interaction between the nodal points of the Eulerian workpiece and the surface of the Lagrangian tool. Since SSTYP=1, the slave entity was identified by a part number; therefore setting SLAVE to the part number 3 designated the tool as the slave. Since MSTYP was set to "0", the master entity was identified through a part set. The MASTER referred to a ${ }^{*}$ SET_PART_LIST (pgs. 208, 211 and 214), which included part numbers of the airmesh and workpiece, designating them as the masters in the contact. The Eulerian and Lagrangian contact coupling was conducted in the normal direction (DIREC=2) through a $3 \times 3 \times 3$ point grid (NQUAD=3) representing the virtual nodes located at the gauss points of the workpiece, which were checked for penetration into the tool.

The definition of the coefficient of friction (FRIC) in this contact algorithm was limited to only one constant value for all applicable areas of contact. The experimental results, which were the basis for the elastic plastic hydrodynamic material model, simplified the stress definition in the material to being a function of strain, while incorporating strain rate, temperature, friction, and all other influences, into the flow curve. Since the hydrodynamic material has the frictional effects embedded in its flow curve, the coefficient of friction was set to " 0.0 " as is shown in Appendix J. Such is not the case for the Johnson-Cook model, which required separate terms in its equation to account for strain, strain rate, and temperature effects. The factor, not incorporated in the Johnson-Cook constitutive model is friction arising from the material contacting the tool; hence it must be accounted for in the definition of workpiece-tool contact. Since the coefficient of friction varies within the contact region, from high values in the sticking region to lower values in the sliding region, it is difficult to estimate a single value to account for the entire contact length. As such, several coefficients of friction were studied for the numerical simulations with the Johnson-Cook material model, ranging from 0.0 to 0.9 for Material Model 1 and from 0.0 to 0.4 for Material Model 2 . 


\subsubsection{Collected Output}

Data specified by *DATABASE_OPTION (pgs. 206, 209 and 212) was collected into ASCII files at intervals of $0.5 \mathrm{~ms}$ (DT). The GLSTAT and MATSUM files gathered information on global statistics, including: energies, external work, velocities of parts and rigid bodies, and time step information. Due to the nature of the Eulerian formulation, it is difficult to detect penetration visually; therefore the SLEOUT file was requested to monitor the sliding interface energy and provides information on the level of penetration (Section 2.2.7). The last ASCII file created was the DBESI file, which recorded interaction forces between the Eulerian workpiece material and the Lagrangian tool.

The *DATABASE_BINARY_D3PLOT plotted output states and data distribution over the geometry, and ${ }^{\circ}$ DATABASE_BINARY_D3THDT recorded time history data for element subsets; both sets of information were collected at an intervals of $0.5 \mathrm{~ms}$ (DT/CYCL).

\subsubsection{Simulation Execution}

The simulation was terminated at $20.0 \mu$ s for simulations incorporating Material Models 1 and 2, and at $30.0 \mu \mathrm{s}$ for simulations incorporating Material Model 3. Termination times were specified by the ENDTIM parameter in the *CONTROL_TERMINATION cards on pgs. 206, 209 and 212. The time step scale factor (TSSFAC) was defined as 0.7 in the *CONTROL_TIMESTEP cards on pgs. 206, 209 and 212.

\subsubsection{Activating Eulerian Formulation}

The use of Eulerian formulation, described in Section 2.2.2, was designated by setting $D C T=2$ in the ${ }^{*}$ CONTROL_ALE cards on pgs. 206, 209 and 212. Advection was performed after every time cycle (NADV=1), using the second-order Van Leer method (METH=2). The parameter AFAC was set to "-1" in order to turn off ALE smoothing, which is controlled by the same card but is not needed for the Eulerian formulation since the mesh is always restored to its initial orientation. Advection was set to start at $0.0 \mathrm{~s}$ (START) and continue throughout the simulation. 


\section{CHAPTER 7}

\section{Finite Element Results I :}

\section{Metal Cutting Model Employing the Lagrangian Element Formulation}

Presented in this chapter are the results of the Lagrangian element formulation, employed in the modeling of metal cutting. All numerical investigations pertaining to the study were simulated with LS-DYNA 960 (Section 2.2.1), on dual Athlon $1.8 \mathrm{GHz}$ processors with one gigabyte of memory.

\subsection{MESH SENSITIVITY ANALYSIS}

As described in Section 4.1, the mesh sensitivity analysis investigated the response of the Lagrangian mesh with four levels of refinement. Mesh (a), with uniform element size was progressively refined in the PDZ and SDZ to form Mesh (b), Mesh (c) and finally the finest mesh, Mesh (d). The processing time of each simulation completed during the mesh sensitivity study is listed in Table 7.1, and detailed results of the analysis are presented thereafter.

\begin{tabular}{|c|c|}
\hline Mesh & Processing Time \\
\hline Mesh (a) & 2 minutes 55 seconds \\
\hline Mesh (b) & 15 minutes 52 seconds \\
\hline Mesh (c) & 1 hours 14 minutes 55 seconds \\
\hline Mesh (d) & 6 hours 37 minutes 7 seconds \\
\hline
\end{tabular}

Table 7.1: Time required to process numerical simulations of each of the four meshes in the mesh sensitivity analysis. 


\subsubsection{Energy Balance}

The balance of energy in a numerical simulation is very important to the validity of the results (Section 2.2.7). Figure 7.1 shows the typical energy balance of the mesh sensitivity investigations, including the external work, total energy, kinetic energy, internal energy, hourglass energy and sliding energy. The total energy was equivalent to the external work, which was a result of the force and displacement applied by the tool (Section 4.1.4). The external work was dispersed into kinetic energy of the tool and workpiece, and internal energy stored in the deformed workpiece. The sliding energy was approximately zero. Hourglass control was effective in preventing hourglass energy or hourglass modes in element deformation. The energy ratio remained constant at 1.0, which confirmed that energy was conserved and that the results are valid with respect to the energy balance.

\subsubsection{Deformation of the Workpiece}

The force applied by the rigid tool imposed a displacement on the workpiece and caused mesh deformation, which was particularly excessive at the tool tip. As described in Section 2.1.3, the material from the tool tip displaces along the PDZ to the chip root; therefore it was expected that most material deformation would occur in the vicinity of the tool tip. Since the Lagrangian mesh is connected to the material, the material deformation resulted in the distortion of the mesh. The more distorted an element becomes the less accurate is its computation capability, and ultimately a maximum state of distortion is reached beyond which the element may "lock-up" and disable further deformation, or invert leading to negative volume. The deformation of the workpiece geometry for all four studied mesh distributions is shown in Figure 7.2, along with the excessively distorted mesh observed at the tool tip. Observations of the mesh adjacent to the tool tip, showed that the smaller the element size, the more distorted the elements became upon material deformation. Mesh (a), which has the coarsest elements, is much less distorted then Mesh (d), which has the smallest elements. Usually a finer mesh distribution is favourable in numerical modeling to ensure more precise calculations, but Figure 7.2 shows that a finer mesh is also more susceptible to distortion when modeling high deformation processes. 


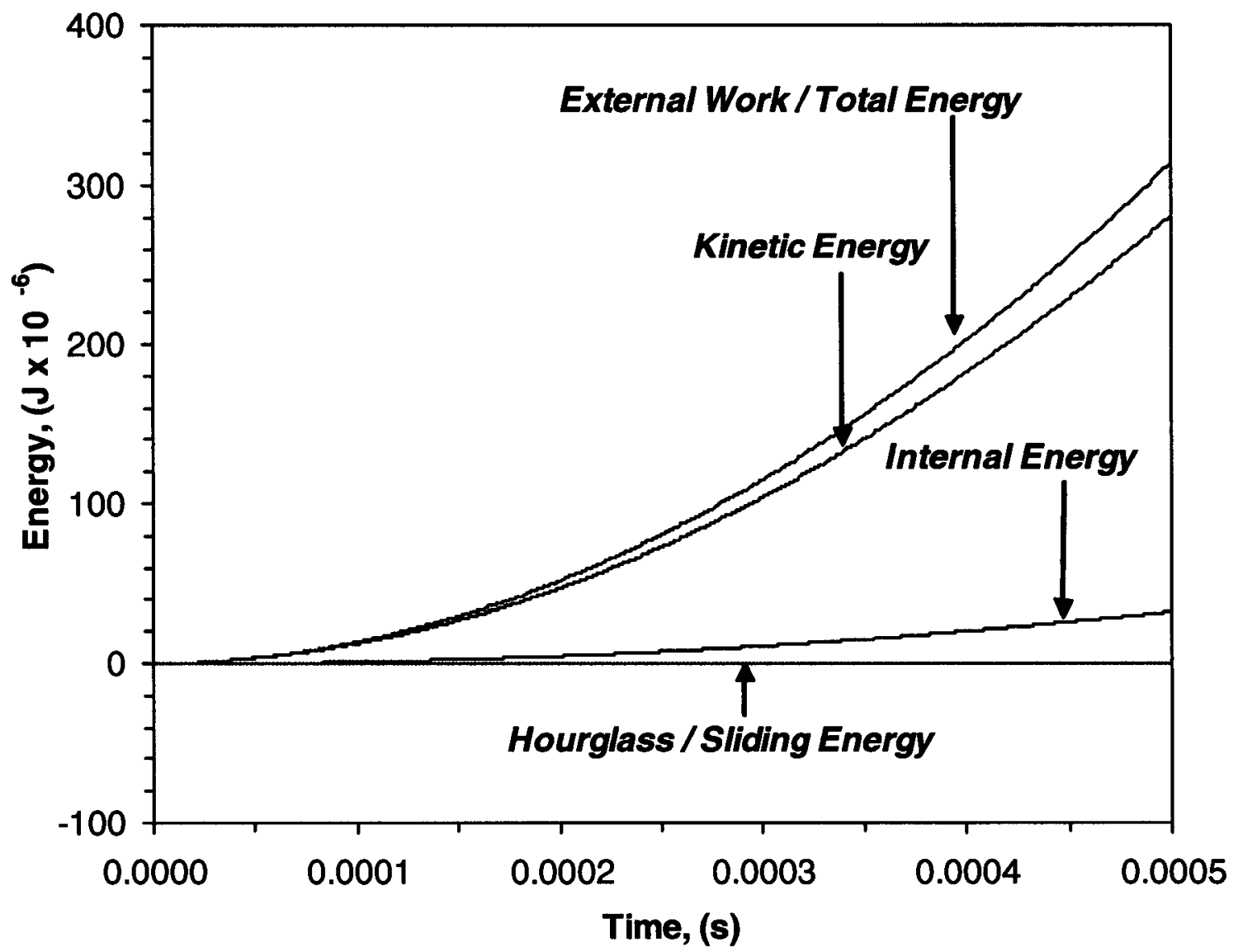

Figure 7.1: Energy balance typical of the mesh sensitivity analysis in the Lagrangian mesh. 

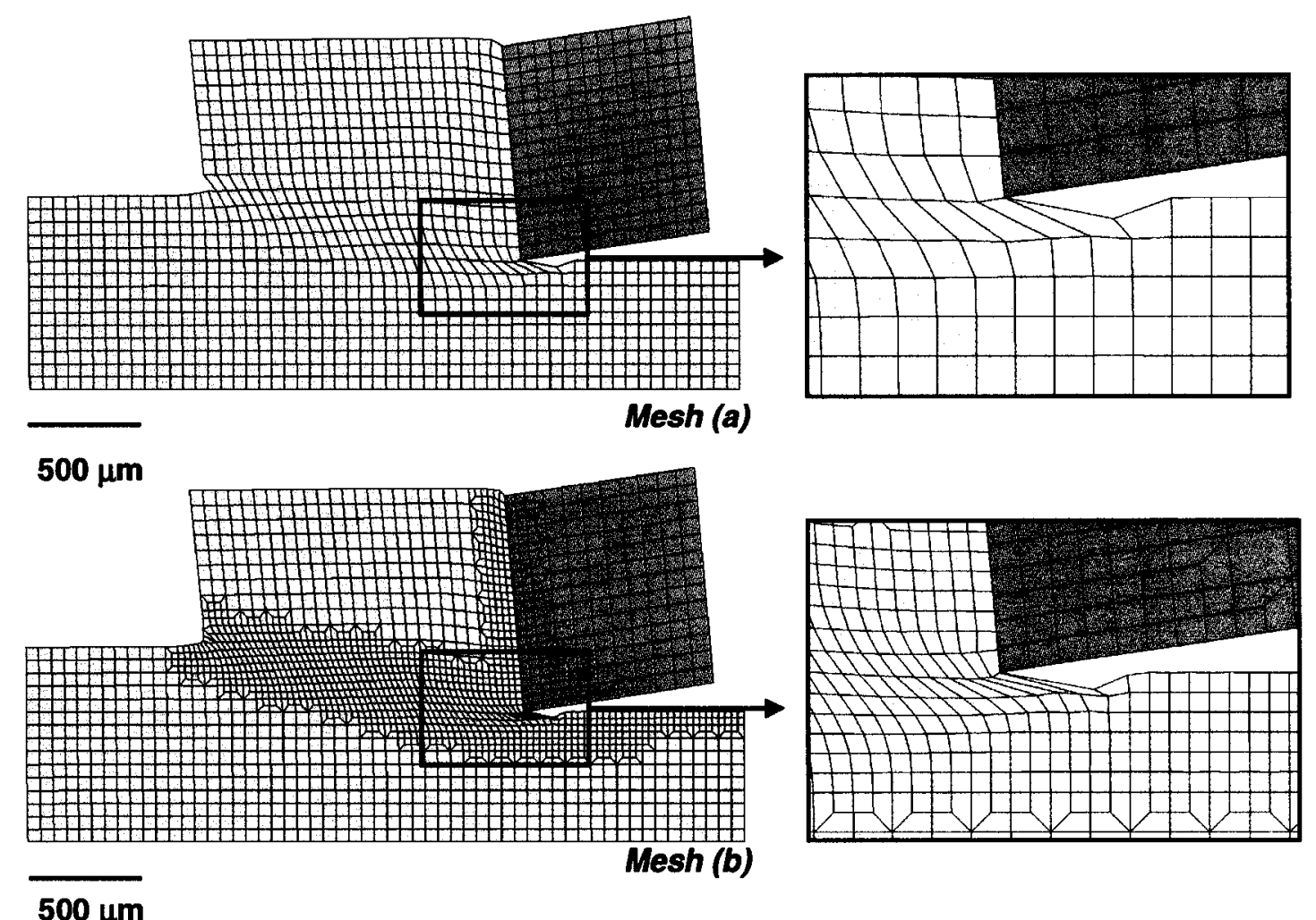

$500 \mu \mathrm{m}$
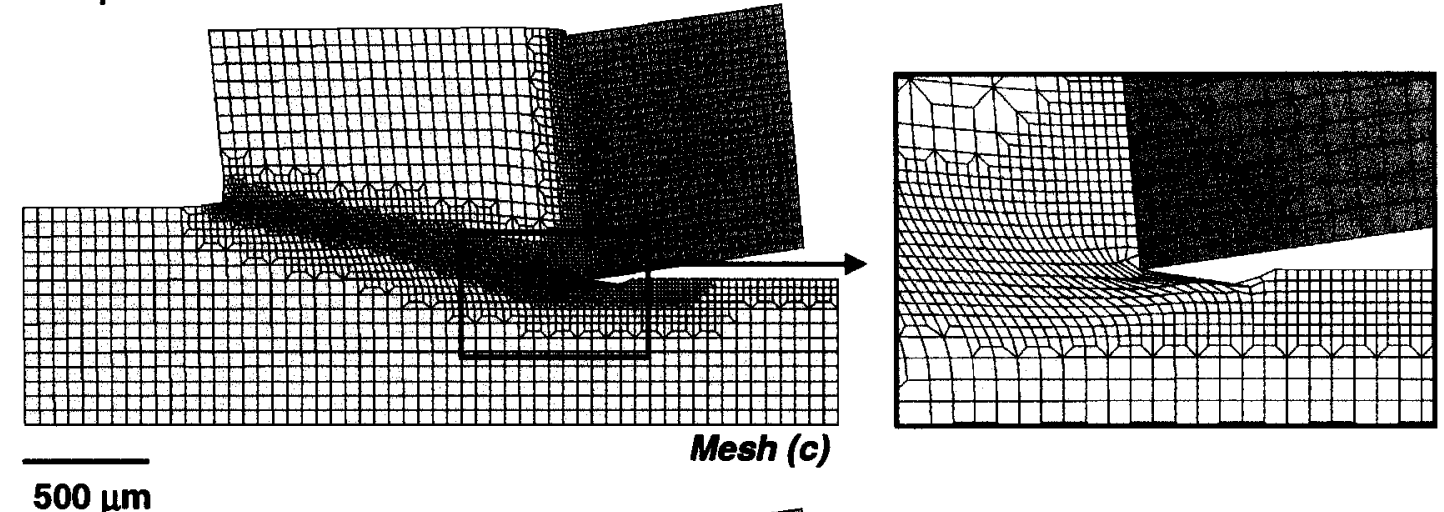

$500 \mu \mathrm{m}$
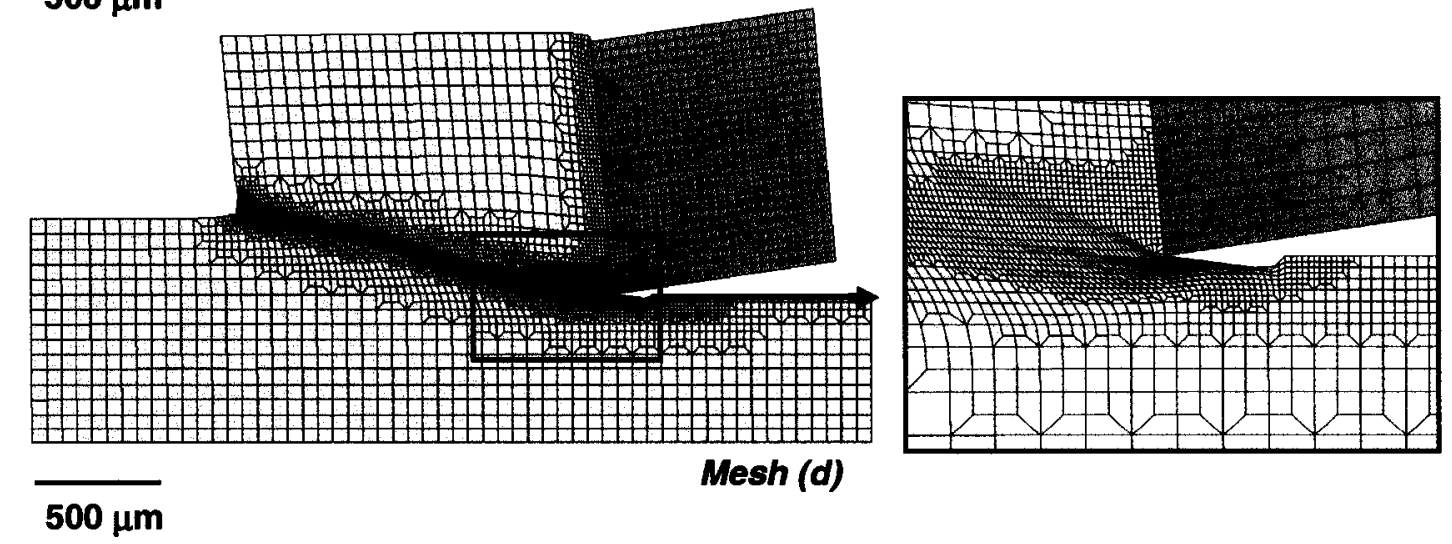

Mesh (d)

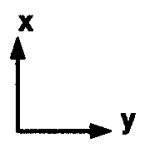

Figure 7.2: Workpiece deformation and mesh distortion around the tool tip for Mesh (a) through (d). 


\subsubsection{Strain Distribution}

The contour plots of the plastic strain in each of the Lagrangian mesh distributions studied, are shown in Figure 7.3. General trends of strain distribution were similar for all cases studied, and showed that strain was concentrated along a region between the tool tip and the chip root. The region of strain concentration is consistent with the location of the PDZ (Section 2.1.3), which is the region of localized plastic deformation or a path along which the material shears from the tool tip to the chip root during chip formation. The approximate width of the region to which the plastic deformation was confined was $320 \mu \mathrm{m}$.

When considering the strain distribution along the deformation zone from the tool tip to the chip root, the maximum strain occurred at the tool tip. It then decreased until reaching a constant value of 0.3 , and again rose towards the chip root reaching an approximate value of 0.5 . The maximum strain value for each mesh studied is listed in Table 7.2. Mesh (d) had a maximum strain of 5.39, which is considerably higher then the maximum strain of 1.19 observed in Mesh (a).

Since the strain is a direct result of element deformation, the maximum strains listed in Table 7.2 are consistent with the levels of deformation illustrated in Figure 7.2. The maximum strain in the tool tip region increases with increasing levels of mesh distortion in the vicinity of the tool tip. The increase in strain at the chip root can also be attributed to mesh distortion, which was not as drastic as at the tool tip but noticeable nonetheless.

\begin{tabular}{|c|c|}
\hline Mesh & Maximum Plastic Strain, $\varepsilon_{p}\left(\mathbf{m m} \cdot \mathbf{m m}^{-1}\right)$ \\
\hline Mesh (a) & 1.19 \\
\hline Mesh (b) & 2.04 \\
\hline Mesh (c) & 3.25 \\
\hline Mesh (d) & 5.39 \\
\hline
\end{tabular}

Table 7.2: Values of maximum plastic strain within the workpiece material for Mesh (a) through (d). 


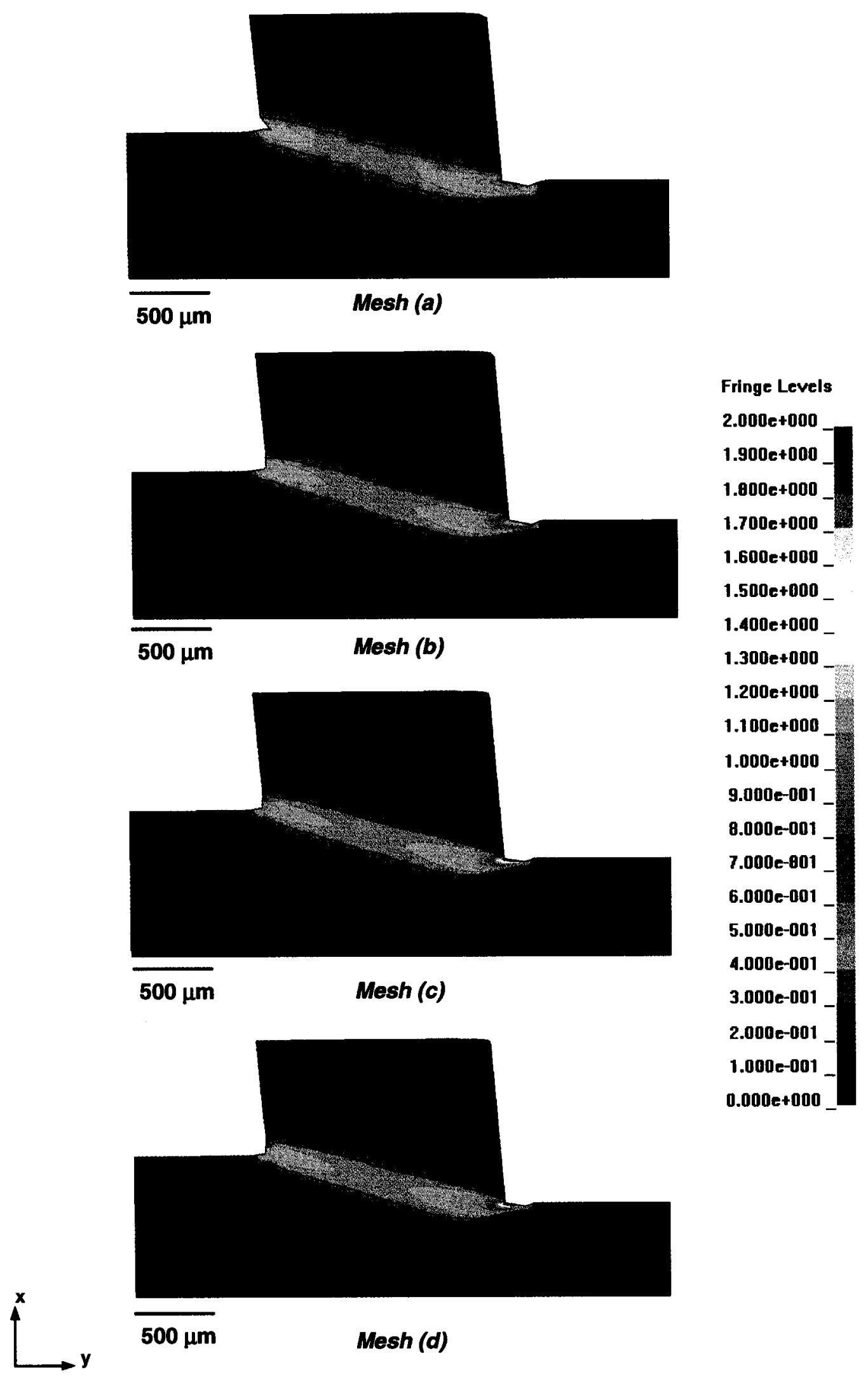

Figure 7.3: Contours of effective plastic strain accumulated in the workpiece, observed in Mesh (a) through (d) of the Lagrangian element formulation study. 


\subsubsection{Stress Distribution}

The contours of stress for Mesh (a) though (d), simulated with the Lagrangian element formulation, are shown in Figure 7.4. Since the stress is directly related to strain through a stress-strain curve described in Section 4.1.3, it was expected that the stress distribution would mimic the strain distribution. This expectation was shown to be true by comparing Figure 7.4 to Figure 7.3 . Much like the strain, the stress was concentrated along a region lying between the tool tip and the chip root, in the locality of the PDZ. The width of the stress concentration region was approximately $200 \mu \mathrm{m}$.

The stress within the PDZ slightly varied, with the maximum stress occurring at the tool tip, increased stresses of approximately $280 \mathrm{MPa}$ at the chip root, and a lower stress of $260 \mathrm{MPa}$ in the bulk of the PDZ between these two extremities. The maximum stress at the tool tip varied from one mesh to the next, and the summary of the maximum stresses in Mesh (a) through (d) are listed in Table 7.3. The stress is directly related to strain and therefore also directly related to the amount of distortion. The maximum amount of distortion observed in Mesh (d) generated the maximum strain and, hence, the maximum stress of 409.3 MPa. The minimal distortion of Mesh (a) generated a minimum stress of only $299.6 \mathrm{MPa}$.

Outside of the PDZ, the stress remained below the yield point since the material deformed only elastically and no plastic strain was accumulated.

\begin{tabular}{|c|c|}
\hline Mesh & Maximum Stress, $\sigma$ (MPa) \\
\hline Mesh (a) & 299.6 \\
\hline Mesh (b) & 345.5 \\
\hline Mesh (c) & 281.1 \\
\hline Mesh (d) & 409.3 \\
\hline
\end{tabular}

Table 7.3: Values of maximum stress, in units of $\mathrm{MPa}$, within the workpiece material for Mesh (a) through (d). 

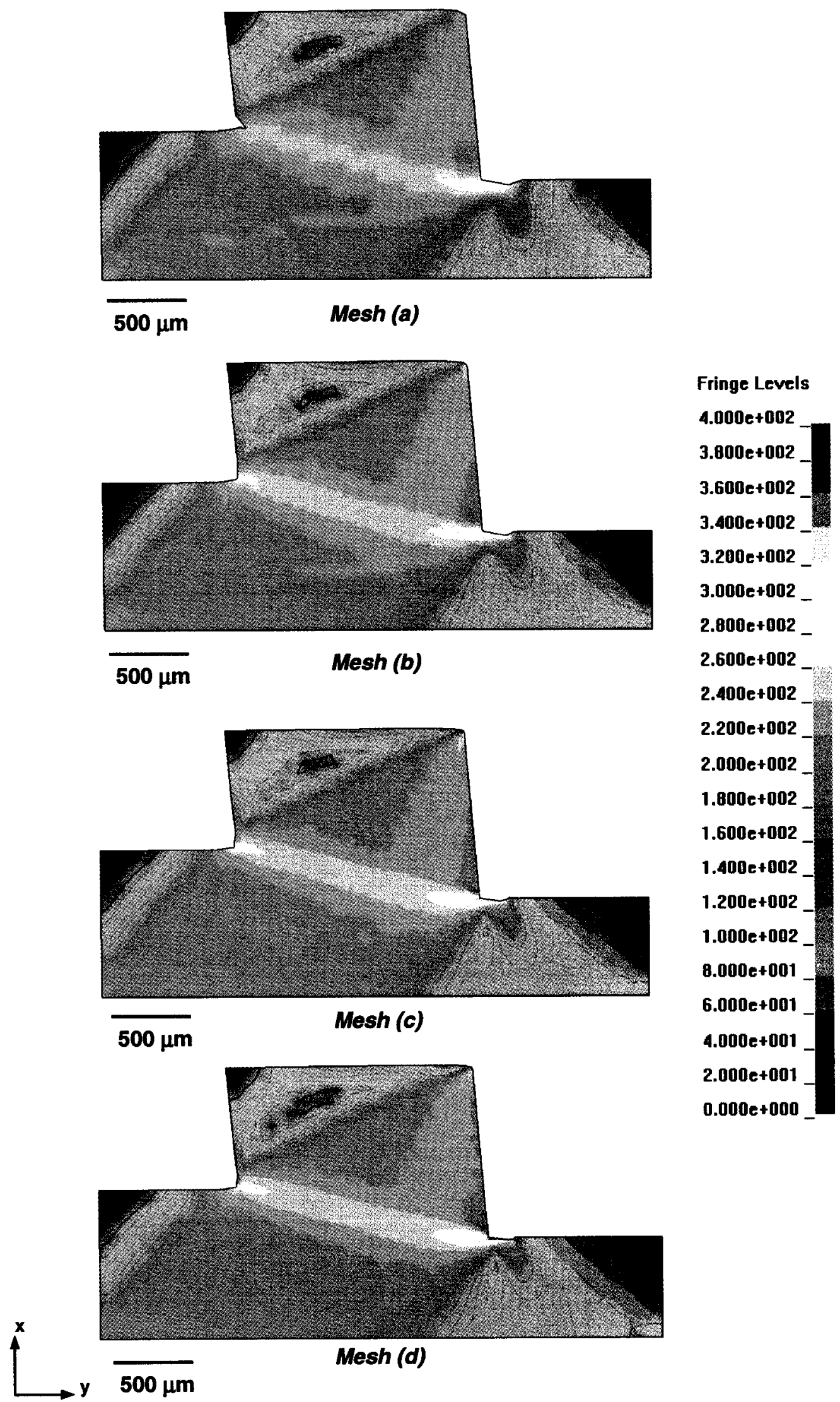

$.000 e+000$

Figure 7.4: Contours of stress, in units of MPa, within the workpiece, observed in Mesh (a) through (d) of the Lagrangian element formulation study. 


\subsection{FULL MESH ADAPTIVITY}

To eliminate the mesh distortion arising in the workpiece material (Figure 7.2), adaptivity was applied to the entire workpiece mesh as described in Section 4.2. The processing time for the simulation with full adaptivity of the workpiece was 11 minutes and 36 seconds.

\subsubsection{Energy Balance}

The energy balance was analyzed to ensure that energy was conserved. Figure 7.5 shows the energy balance was maintained throughout the simulation with full workpiece adaptivity. The total energy of the system was equivalent to the external work. The total energy was composed of internal energy stored in the deformed workpiece and kinetic energy of the moving tool and workpiece. The sliding and hourglass energies were negligible, confirming that neither hourglass deformation nor penetration occurred. The energy ratio was 1.0 throughout the simulation, which established the results are valid with respect to the energy balance.

\subsubsection{Deformation of the Workpiece}

The deformation of the workpiece prior to adaptivity was identical to that of Mesh (a) in the study of the Lagrangian element formulation. Figure 7.6 (a) shows the mesh geometry just prior to the adaptivity, while Figure 7.6 (b) shows the mesh in the consecutive time step, after adaptivity was activated. It is evident by comparing Figures 7.6 (a) and 7.6 (b), that each element of the original mesh was subdivided into four elements generating a much finer mesh. Throughout the remainder of the simulation, the mesh adaptivity followed every time step and was successful in reducing excessive mesh distortion (or preventing it) at the tool tip, as shown in Figure 7.6 (c). The most significant problem arising from the simulation was the loss of nodal constraints upon the activation of adaptivity. When the mesh was subdivided through adaptivity, an entirely new mesh was generated and the constraints placed on the original mesh were not retained in the new mesh. Constraints were placed on the nodes at the bottom of the workpiece restricting their motion in all DOF. After mesh 


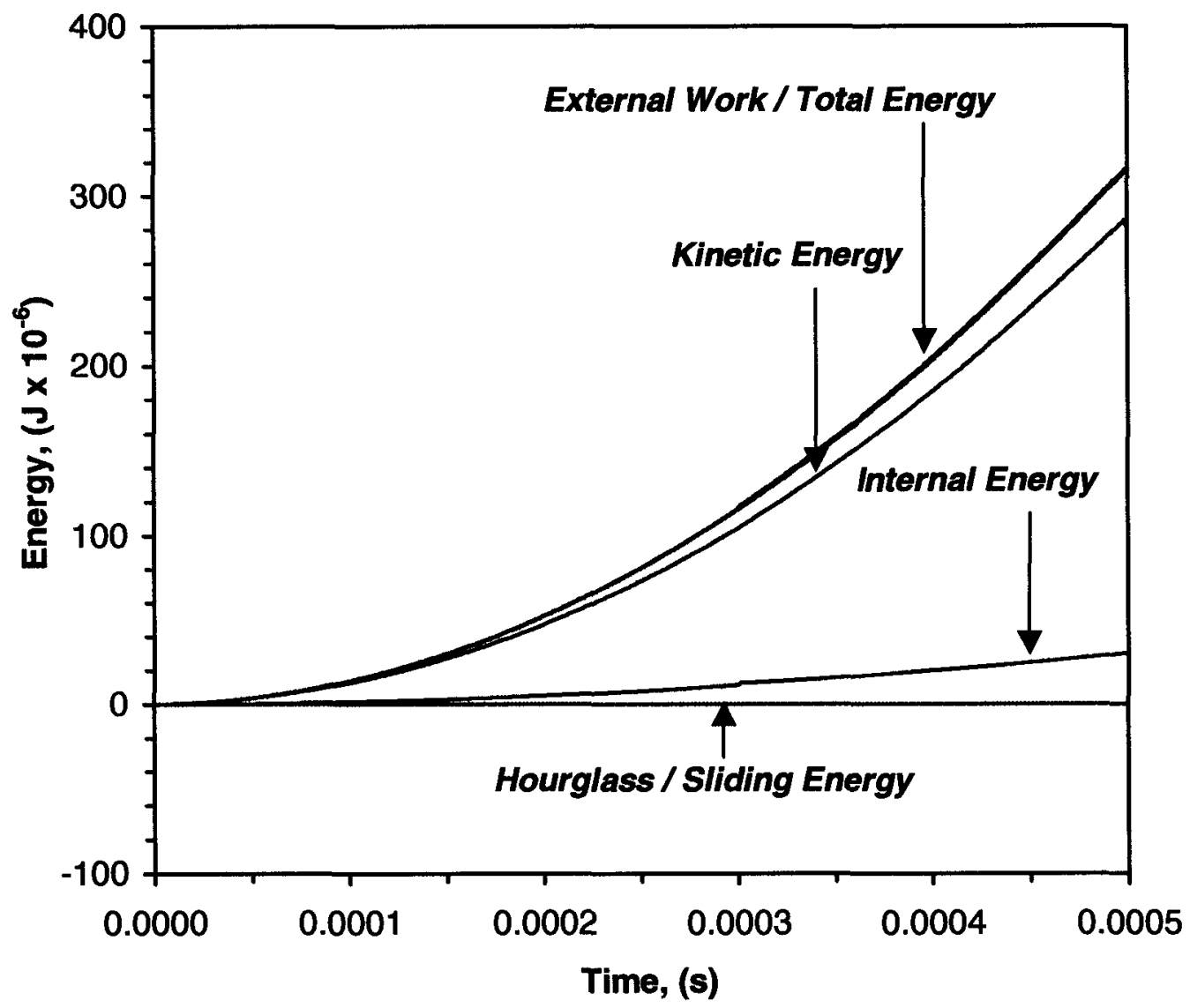

Figure 7.5: Energy balance typical of the mesh sensitivity analysis of the Lagrangian mesh with adaptivity. 

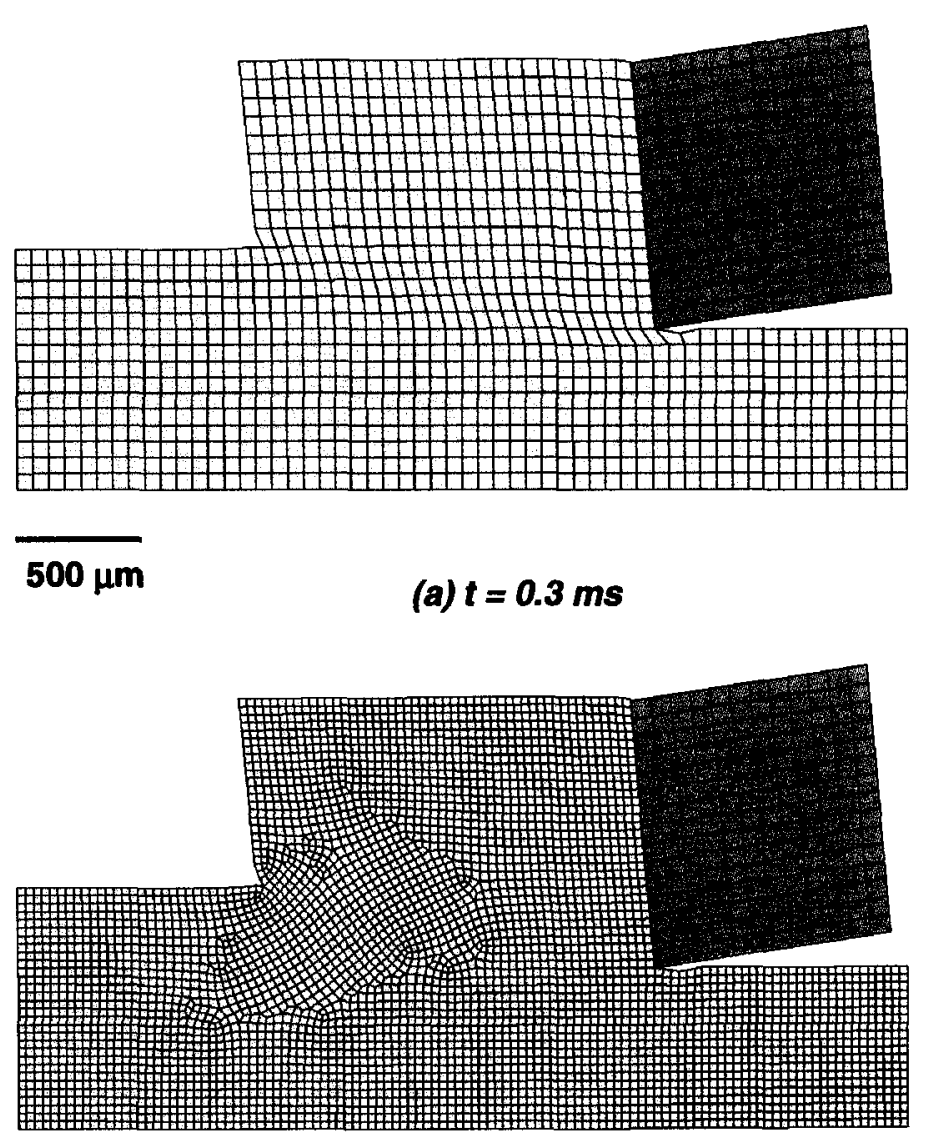

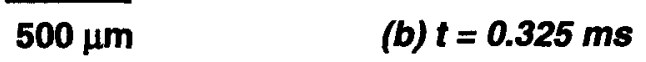
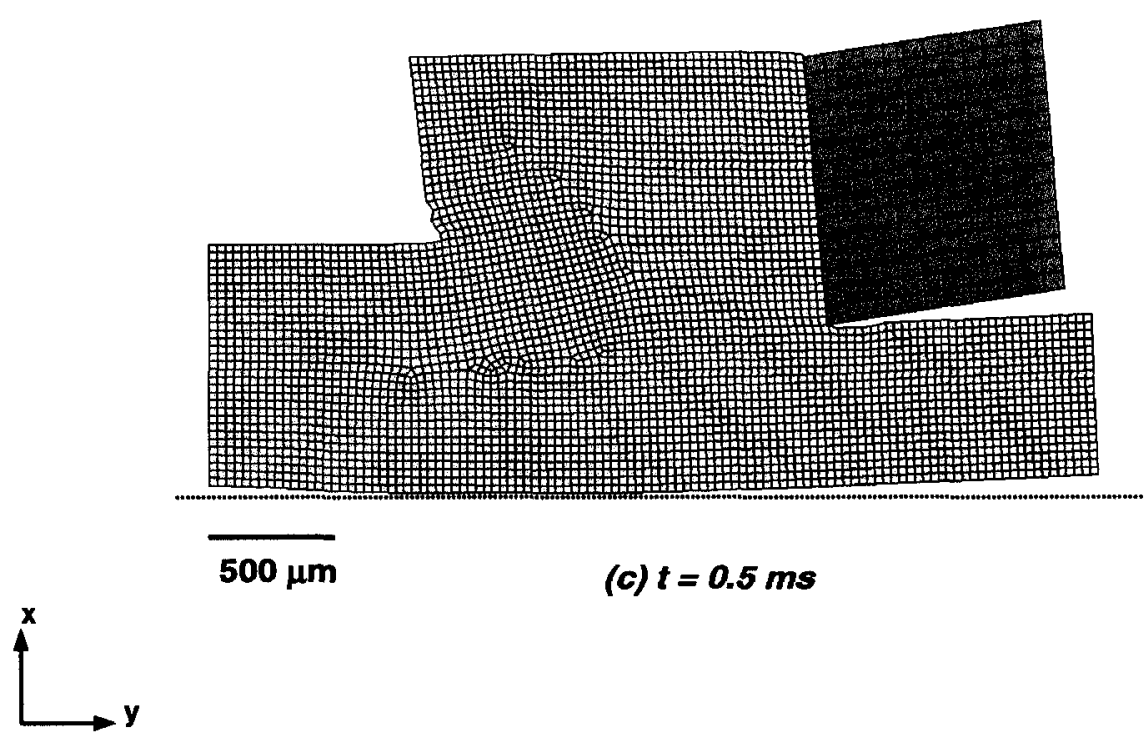

$500 \mu \mathrm{m}$

(c) $t=0.5 \mathrm{~ms}$

Figure 7.6: Progression of mesh/material deformation at a time of: (a) $0.3 \mathrm{~ms}$, (b) $0.325 \mathrm{~ms}$, and (c) termination time of $0.5 \mathrm{~ms}$. Mesh adaptivity was activated at $0.3 \mathrm{~ms}$. 
adaptivity was activated the bottom of the workpiece was no longer restricted. At termination time, definite bending can be observed in the workpiece by examining the bottom of the workpiece in Figure 7.6 (c).

\subsubsection{Strain Distribution}

The strain distribution in the workpiece at termination time is shown in Figure 7.7. As occurred without mesh adaptivity, the concentration of strain was around the PDZ. The highest strains were localized at the chip root and tool tip and ranged between 0.5 and 0.6 . The remainder of the PDZ accumulated strain of approximately 0.3 . Since the adaptivity was successful in reducing element distortion, the maximum strain at the tool tip was only 0.745 , which is significantly lower than the strain of 5.39 occurring in the highly distorted Mesh (d) and discussed in Section 7.1.3. As a result of the constraint loss, the workpiece underwent bending and additional strain developed from the PDZ towards the bottom of the workpiece. The ability of the workpiece to relieve the applied force through bending, likely contributed to the comparatively lower strains observed in this workpiece, as opposed to the strains illustrated in Figure 7.3.

\subsubsection{Stress Distribution}

The distribution of stress within the adapted workpiece is shown in Figure 7.8, in units of MPa. As expected the maximum stress of $270.6 \mathrm{MPa}$ occurred at the tool tip consistently with the location of maximum strain. The concentration of stress was located within the PDZ and extended towards the bottom of the workpiece as a result of bending, the values of stress in this area were approximately $240-260 \mathrm{MPa}$. The stresses in regions of elastic deformation remained below the yield stress value of 199.3 MPa. 
Figure 7.7: Contours of effective plastic strain, observed in the adapted workpiece.

\author{
Fringe Levels \\ $4.000 e+002$ \\ $3.800+002$ \\ $3.600 e+002$ \\ $3.400 e+002$ \\ $3.200 e+002$ \\ $3.000 \mathrm{e}+002$ \\ $2.800 \mathrm{e}+002$ \\ $2.600 e+002$ \\ 2.400e+002 \\ $2.200 e+002$ \\ $2.000 e+002$ \\ 1.800e+002 \\ $1.600 \mathrm{e}+002$ \\ $1.400 \mathrm{e}+002$ \\ $1.200 e+002$ \\ $1.000 e+002$ \\ $8.000 e+001$ \\ 6.000e+001 \\ $4.000 \mathrm{e}+001$ \\ $2.000 e+001$ \\ $0.000 e+000$
}

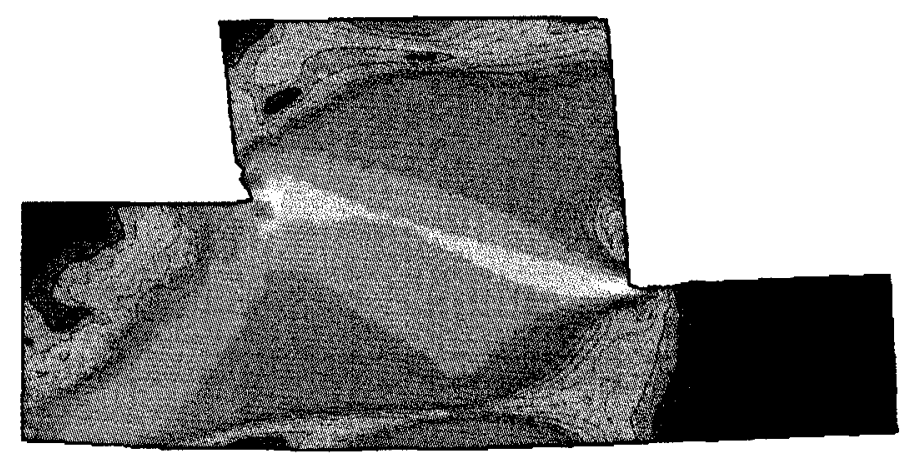

Figure 7.8: Contours of von Mises stress, in units of MPa, within the adapted workpiece. 


\subsection{PARTIAL MESH ADAPTIVITY}

The procedures to partially adapting the workpiece mesh are outlined in Section 4.2. The partial adaptivity was sought out in an effort to shorten simulation time by adapting only the part of the mesh that was the most distorted under the tool force, which was shown to be the tool tip in Figure 7.2. Adapting only a fraction of the mesh was also an attempt to resolve a problems arising from full mesh adaptivity, or the loss of constraints at the bottom of the workpiece causing workpiece bending. The processing time for the simulation with partial adaptivity of the workpiece was 4 minutes and 5 seconds.

\subsubsection{Deformation of the Workpiece}

The deformation of the workpiece and mesh was the first to be analyzed after the simulation was completed, and it is illustrated in Figure 7.9. Within the first time step, the fraction of the mesh that was set to be adapted was regenerated into a new finer mesh. Since an entirely new mesh was generated and was made up of all new nodes, it was no longer merged with the remainder of the mesh as was before adaptivity occurred. Since nodal connectivity was not conserved, the adapted part completely separated from the remainder of the workpiece. The analysis was deemed inconsequential and no further results of this simulation were investigated. 


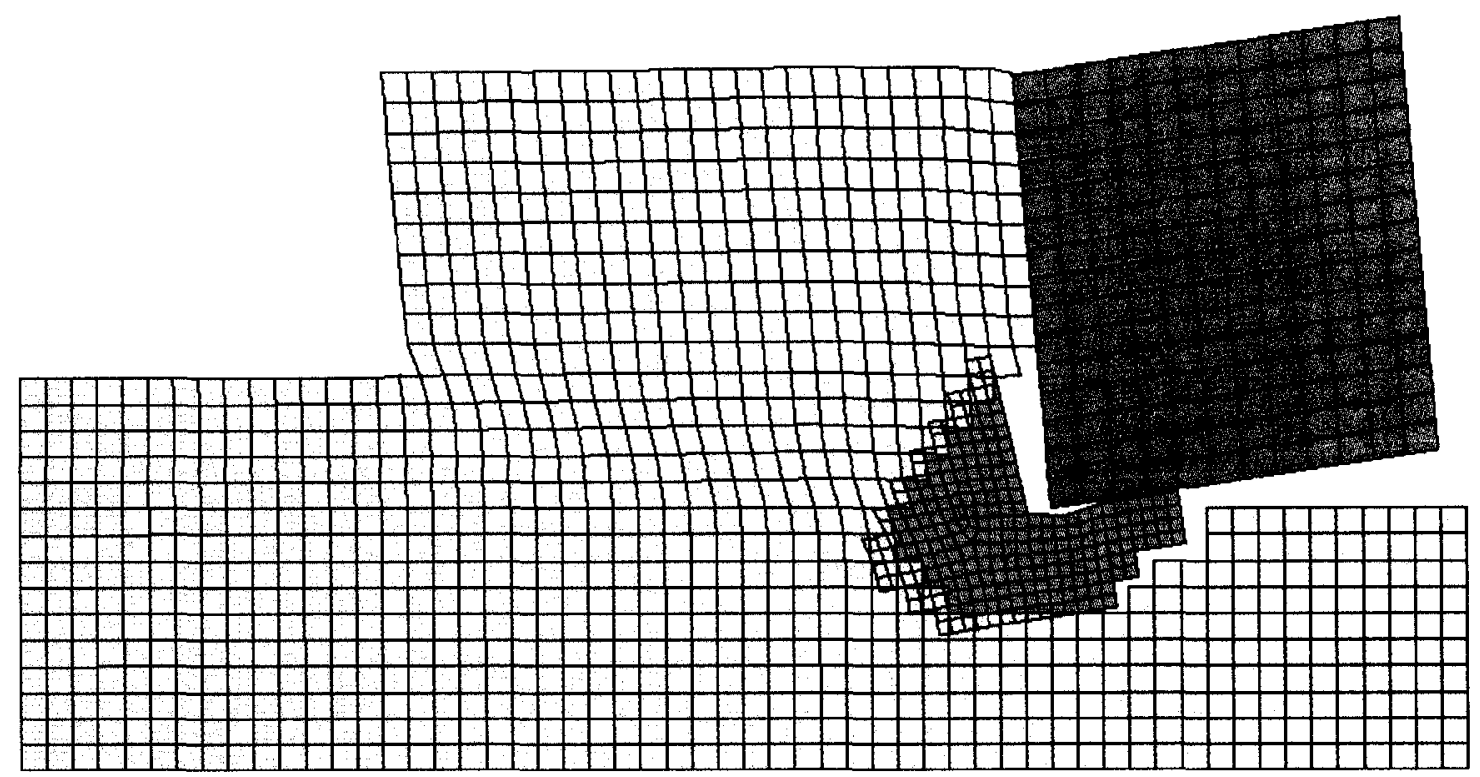

$500 \mu \mathrm{m}$

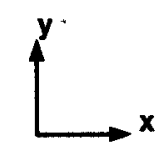

Figure 7.9: Deformation of the mesh with partial adaptivity. The adapted region has separated from the remainder of the mesh. 


\section{CHAPTER 8}

\section{Finite Element Results II :}

\section{Metal Cutting Model Employing the ALE Element Formulation}

Presented in this chapter are the results of the investigation of the Arbitrary Lagrangian-Eulerian (ALE) element formulation, applicable to the modeling of metal cutting. As previously stated, all simulations were performed using LS-DYNA 960 and dual Athlon $1.8 \mathrm{GHz}$ processors with one gigabyte of memory.

\subsection{STEADY STATE CHIP FORMATION}

The procedures for the study of steady state chip formation with an ALE element formulation are summarized in Section 5.1. The simulation was stopped at $0.333 \mathrm{~ms}$, before the defined termination time, due to complications in the analysis. At the point of termination, the processing time was approximately 40 minutes.

\subsubsection{Energy Balance}

The energy balance observed in modeling of the steady state chip formation through ALE element formulation is shown in Figure 8.1, and resembled those observed in the Lagrangian studies discussed in Sections 7.1.1 and 7.2.1. The external work was equivalent to the total energy. The total energy was composed of internal energy stored in the deformed workpiece material and of the kinetic energy accumulated by the motion of the tool and workpiece. Sliding energy was negligible. The maximum hourglass energy during the simulation was observed at $0.3 \mathrm{~ms}$, it remained at a value of less then $5 \%$ of the total energy and therefore it was deemed acceptable. 


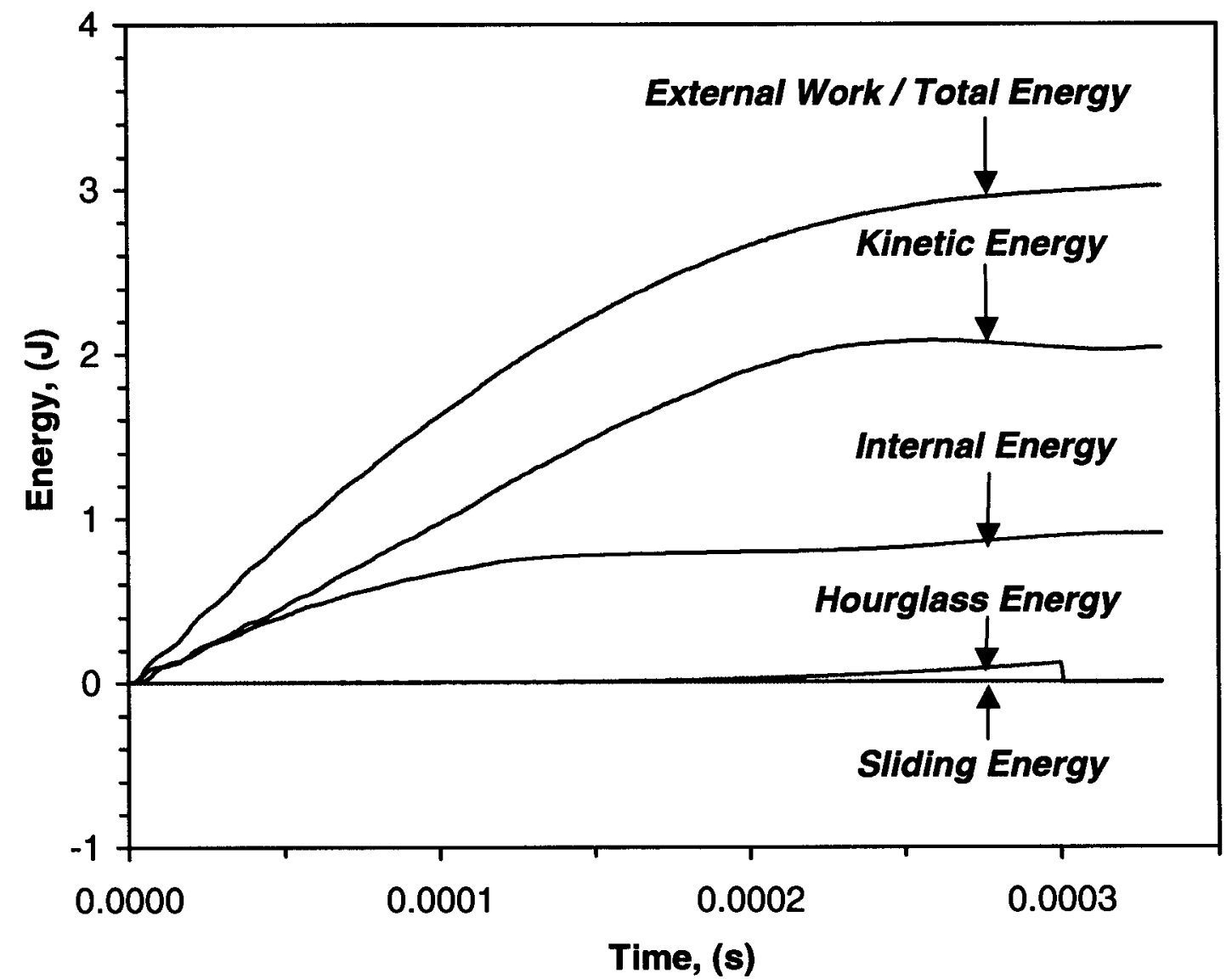

Figure 8.1: Energy balance of the steady state chip formation simulation using the ALE element formulation. 


\subsubsection{Deformation of the Workpiece}

The motion applied to the tool, resulted in the displacement of the workpiece and therefore the deformation of its mesh. The material deformation was monitored throughout the duration of the simulation. At approximately $0.15 \mathrm{~ms}$, a problem arose in the material response. The bottom of the workpiece became free of the constraints placed upon it, and it bent to relieve the force being applied by the tool. This problem was detected at $0.333 \mathrm{~ms}$, at which time the simulation was terminated prematurely. Figure 8.2 (a) shows the mesh deformation at a time of $0.15 \mathrm{~ms}$, just before the bottom constraints were released. Since the mesh cross-section resembles that of Mesh (b) used in the Lagrangian study (Section 7.1), comparing the distortion of Mesh (b) in Figure 7.2 and distortion in Figure 8.2 (a) shows definite improvement in controlling mesh distortion. This indicates that ALE smoothing did occur through the simulation. The mesh distortion at termination time $(0.333 \mathrm{~ms})$ is shown in Figure $8.2(\mathrm{~b})$. The problems arising in this simulation are illustrated in this figure. The constraints at the bottom of the workpiece had completely vanished, and the workpiece had bowed. Despite several manipulations to the input code, including adjusting the time step and the ALE smoothing weight factors, the problem persisted.

\subsubsection{Strain Distribution}

The strain distribution of the steady state chip formation using the ALE element formulation is shown in Figure 8.3. Strain was analyzed at $0.15 \mathrm{~ms}$ and $0.333 \mathrm{~ms}$, before and after the loss of constraints on the bottom of the workpiece. The distribution differs significantly from those observed in the Lagrangian study, shown in Figures 7.3 and 7.7. Previously, the concentration of strain was observed around the PDZ, but Figure 8.3 shows that in this case the strain was concentrated at the tool rake face or in the SDZ. The maximum strain observed was 2.68 and 5.87 at $0.15 \mathrm{~ms}$ and $0.333 \mathrm{~ms}$, respectively. The maximum strain occurred along the rake face approximately $250 \mu \mathrm{m}$ above the tool tip. Increased strains were observed directly adjacent to the rake face of the tool and decreased away from the tool tip, which was likely the result of the friction applied between the tool and workpiece. The average width of the SDZ was predicted to be 


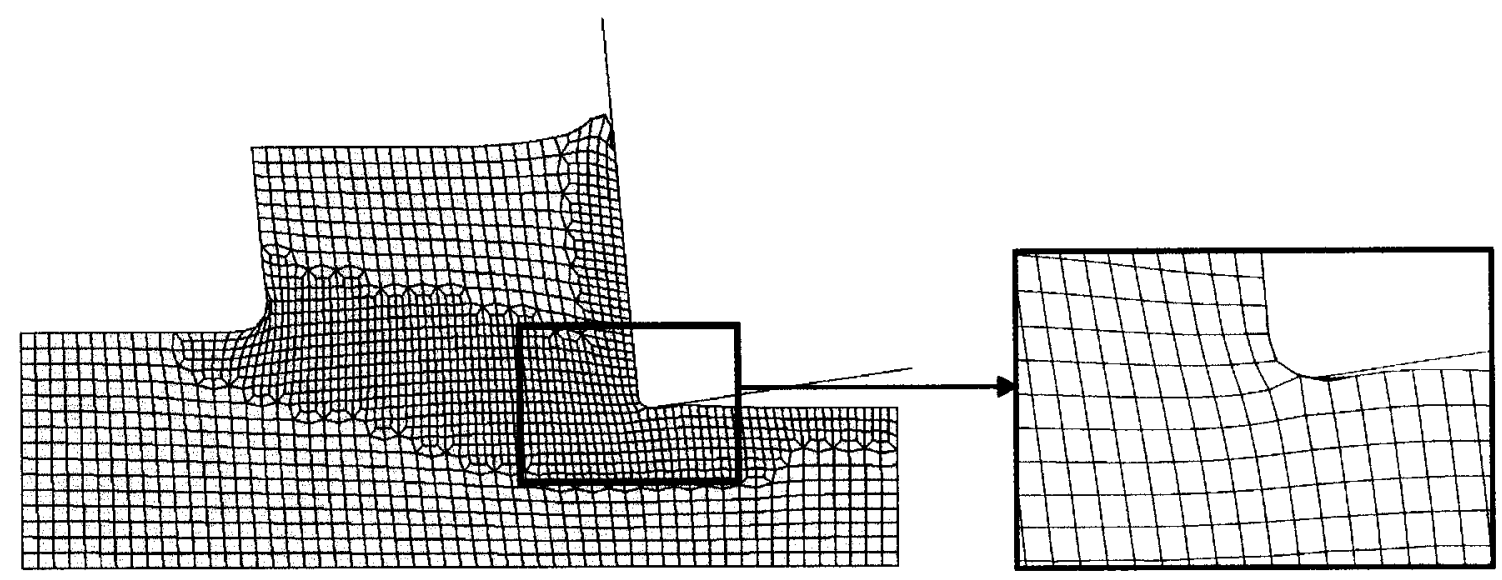

$500 \mu \mathrm{m}$

(a) $t=0.15 \mathrm{~ms}$

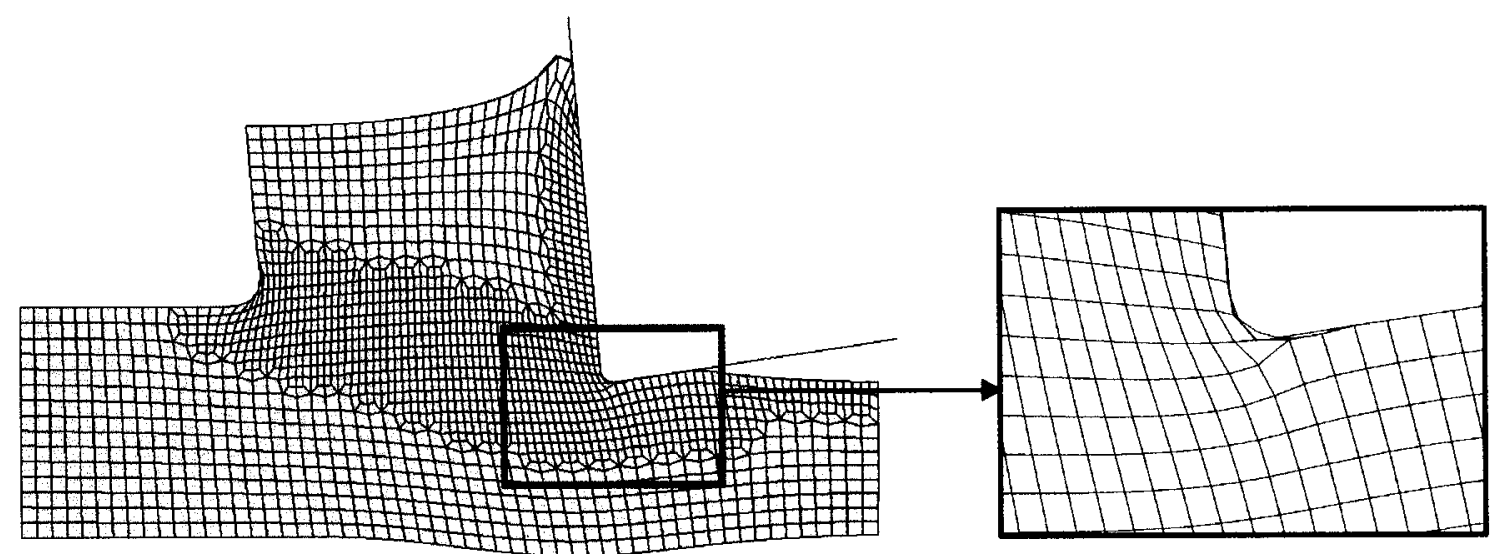

$500 \mu \mathrm{m}$

(b) $t=0.333 \mathrm{~ms}$

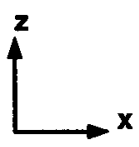

Figure 8.2: Deformation of the workpiece modeled with an ALE mesh at: (a) $t=0.15 \mathrm{~ms}$, and (b) $\mathrm{t}=0.333 \mathrm{~ms}$. 
approximately $200 \mu \mathrm{m}$. At the termination time of $0.333 \mathrm{~ms}$, strains also developed at the bottom of the workpiece where the most bending had occurred. These strains were relatively small compared to those observed in the SDZ.

\subsubsection{Stress Distribution}

The stress distribution of the steady state chip formed by the ALE element formulation is presented in Figure 8.4. As with the strains, the stress was analyzed at $0.15 \mathrm{~ms}$ and $0.333 \mathrm{~ms}$, before and after the loss of constraints on the bottom of the workpiece. The stress distribution was very similar to the strain distribution, due to the fact that the two are related through a simple binary stress-strain flow curve (Section 5.1.3). The maximum stresses were observed at the locations of maximum strains, approximately $250 \mu \mathrm{m}$ above the tool tip. The stresses reached values of 478.1 MPa and $797.6 \mathrm{MPa}$ at a time of $0.15 \mathrm{~ms}$ and $0.333 \mathrm{~ms}$, respectively; the stresses were higher then those in the Lagrangian study due to the use of different material models in the two cases. The stresses were localized in the SDZ with maximum stresses occurring at the rake face and decreasing away from the rake face. At the termination time, Figure 8.4 (b) reveals that small stresses also developed at the bottom of the workpiece due to the bending in the workpiece.

\subsection{INCIPIENT CHIP FORMATION}

The procedures for the study of incipient chip formation with an ALE element formulation are summarized in Section 5.2. Despite numerous attempts to adjust the FE input file and optimize ALE parameters, the simulation persistently terminated with an error before the termination time was reached, stating negative volume elements. The error was due to the extreme deformation of elements in the workpiece. The best of the numerous attempts was one which terminated at a time of $0.54 \mathrm{~ms}$ after 2 minutes and 38 seconds of processing time. The results of this simulation are summarized in the following sections. 


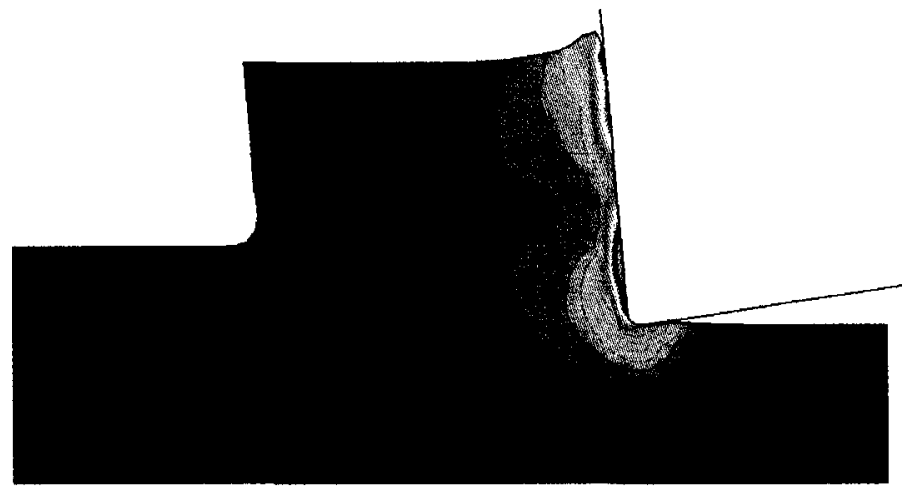

$500 \mu \mathrm{m}$

(a) $t=0.15 \mathrm{~ms}$

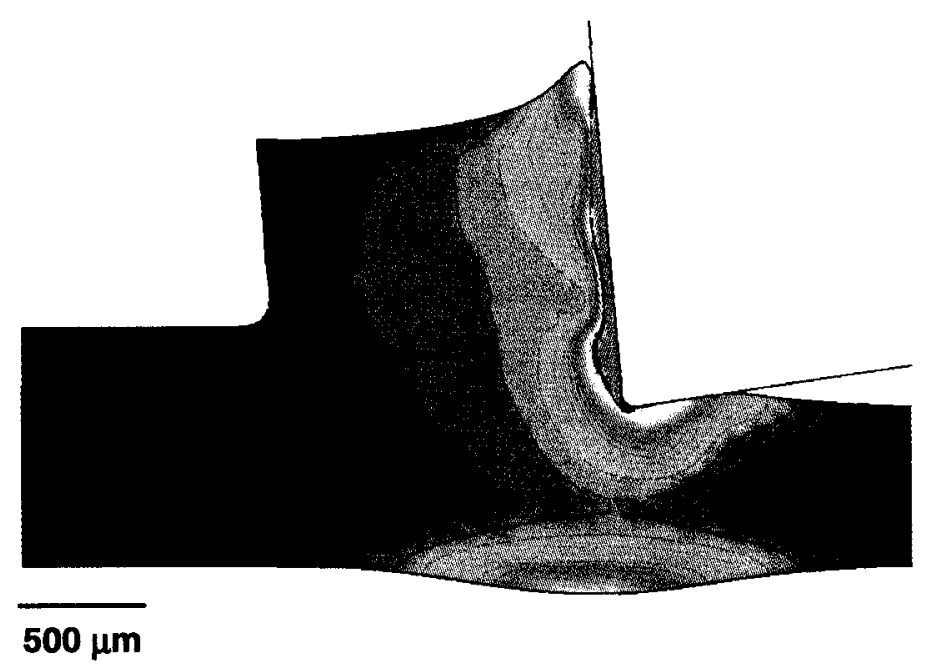

(b) $t=0.333 \mathrm{~ms}$

$500 \mu \mathrm{m}$
Fringe Levels

$2.000 e+000$

$1.900 e+000$

$1.800 e+000$

$1.700 e+000$

$1.600 e+000$

$1.500 e+000$

$1.400 e+000$

$1.300 e+000$

$1.200 e+000$

$1.100 e+000$

$1.000 e+000$

9.000e-001

8.000e-001

$7.000 e-001$

6.000e-001

5.000e-001

4.000e-001

3.000e-001

2.000e-001

1.000e-001

$0.000 e+000$

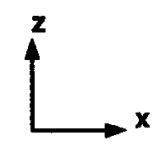

Figure 8.3: Strain distribution in the workpiece modeled with an ALE element formulation at: (a) $\mathrm{t}=0.15 \mathrm{~ms}$, and (b) $\mathrm{t}=0.333 \mathrm{~ms}$. 


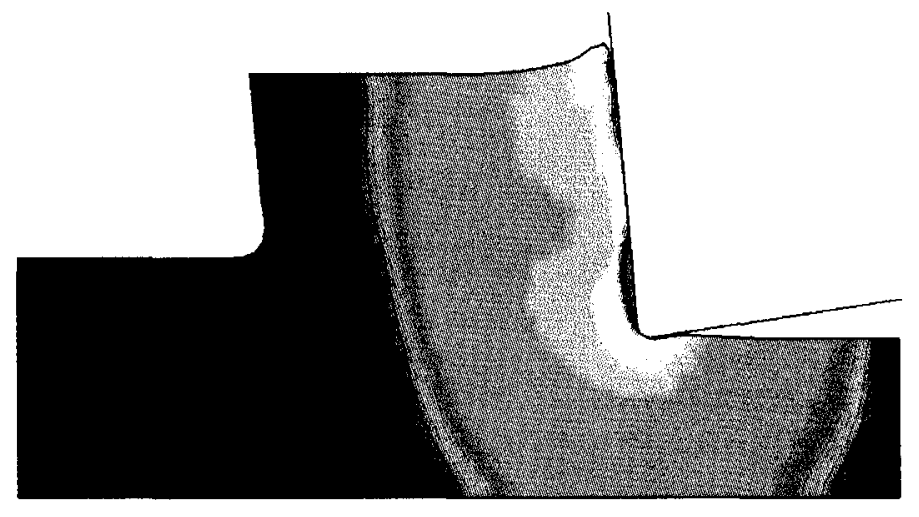

$\overline{500 \mu \mathrm{m}}$

(a) $t=0.15 \mathrm{~ms}$

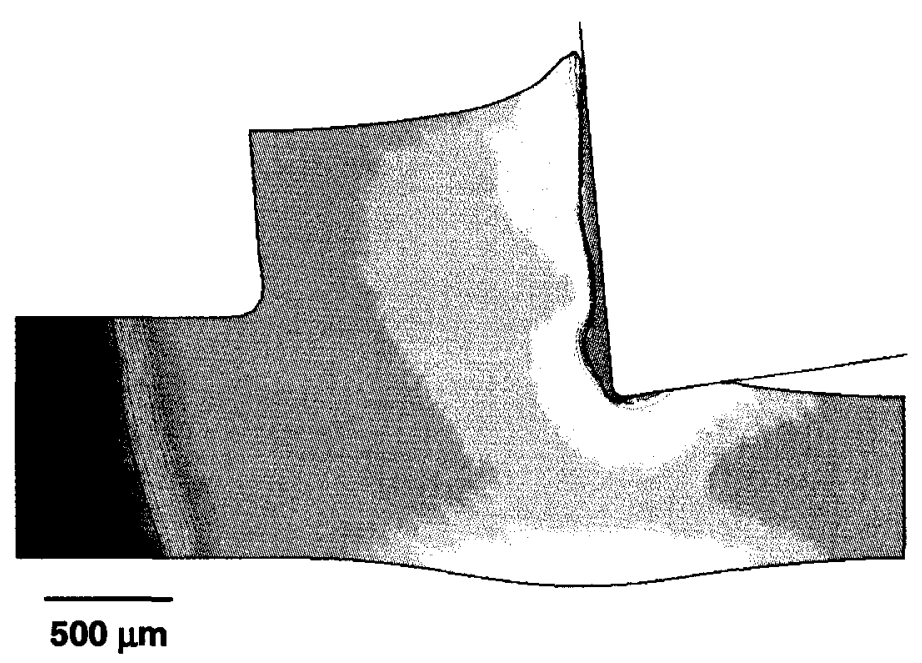

(b) $t=0.333 \mathrm{~ms}$
Fringe Levels

4.000e+002

$3.800 e+002$

$3.600 e+002$

$3.400 e+002$

$3.200 e+002$

$3.000 e+002$

$2.800 c+002$

2.600e+002

$2.400 e+002$

2.200e+002

$2.000 e+002$

$1.800 e+002$

$1.600 e+002$

1.400e+002

1.200e+002

$1.000 e+002$

$8.000 e+001$

$6.000 e+001$

4.000e+001

$2.000 e+001$

$0.000 e+000$

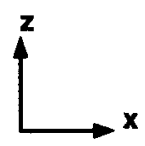

Figure 8.4: Distribution of stress in units of MPa, for the workpiece modeled with an ALE element formulation at: (a) $\mathrm{t}=0.15 \mathrm{~ms}$, and (b) $\mathrm{t}=0.333 \mathrm{~ms}$. 


\subsubsection{Energy Balance}

The energy balance attained during the modeling of the incipient chip formation with an ALE element formulation, is shown in Figure 8.5. The most significant problem detected through the energy balance was the high negative sliding interface energy, which was approximately $25 \%$ of total energy at termination time. Due to the high velocities applied in the process, the tool had the tendency to penetrate into the workpiece at the tool tip area and made the sliding energy very difficult to control. Despite efforts to optimize the contact parameters, the shown negative sliding energy was the minimum sliding energy that could be attained. Manipulation of the hourglass control parameters was successful in keeping the hourglass energy at nearly zero throughout the simulation. The kinetic energy was significantly higher then the internal energy, due to the extremely high velocity of the tool and the limited workpiece deformation occurring prior to error termination. The external work and total energy were equivalent to each other up to a time of $0.2 \mathrm{~ms}$, but the external work increasingly exceeded the total energy thereafter. At termination time the total energy was approximately $90 \%$ of the external work.

\subsubsection{Deformation of the Workpiece}

The motion of the tool resulted in the deformation of the workpiece and therefore the deformation of its mesh. The deformation of the mesh was monitored during the simulation, and is illustrated in Figure 8.6. Penetration of the tool into the workpiece was evident at a time of $0.25 \mathrm{~ms}$ and can be seen in Figure 8.6 (a) at the rake face of the tool. Figure 8.6 (b), which presents the deformation at $0.5 \mathrm{~ms}$, shows not only penetration of the tool into the workpiece but also significant workpiece mesh distortion at the rake face and at the tool tip. The negative volume elements at the time of termination were elements adjacent to the rake face. It was apparent from observing the mesh deformation in Figure 8.6 that the generated ALE mesh was incapable of controlling the distortion of the workpiece mesh under the applied metal cutting conditions. 


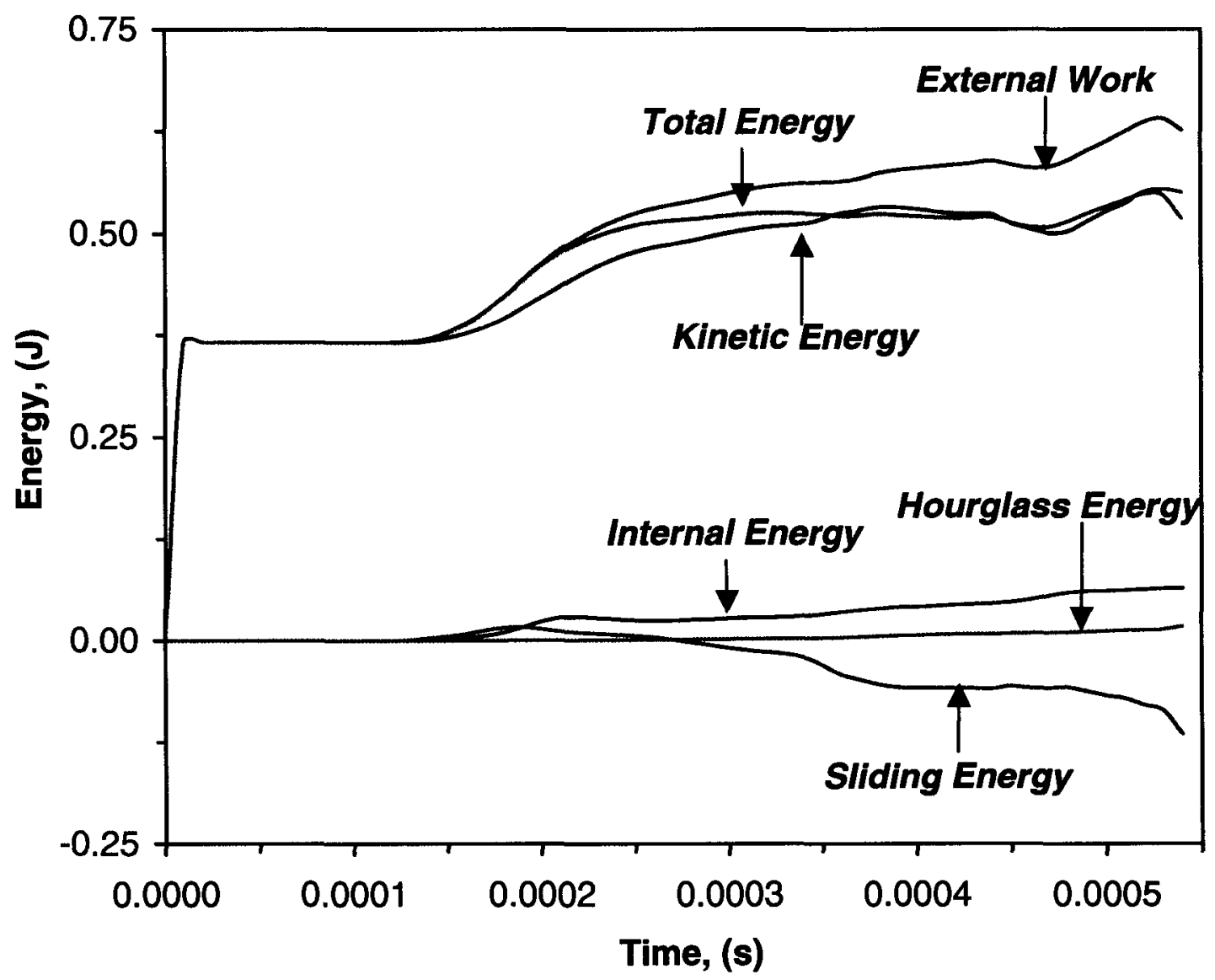

Figure 8.5: Energy balance of the incipient state chip formation simulation using the ALE element formulation. 


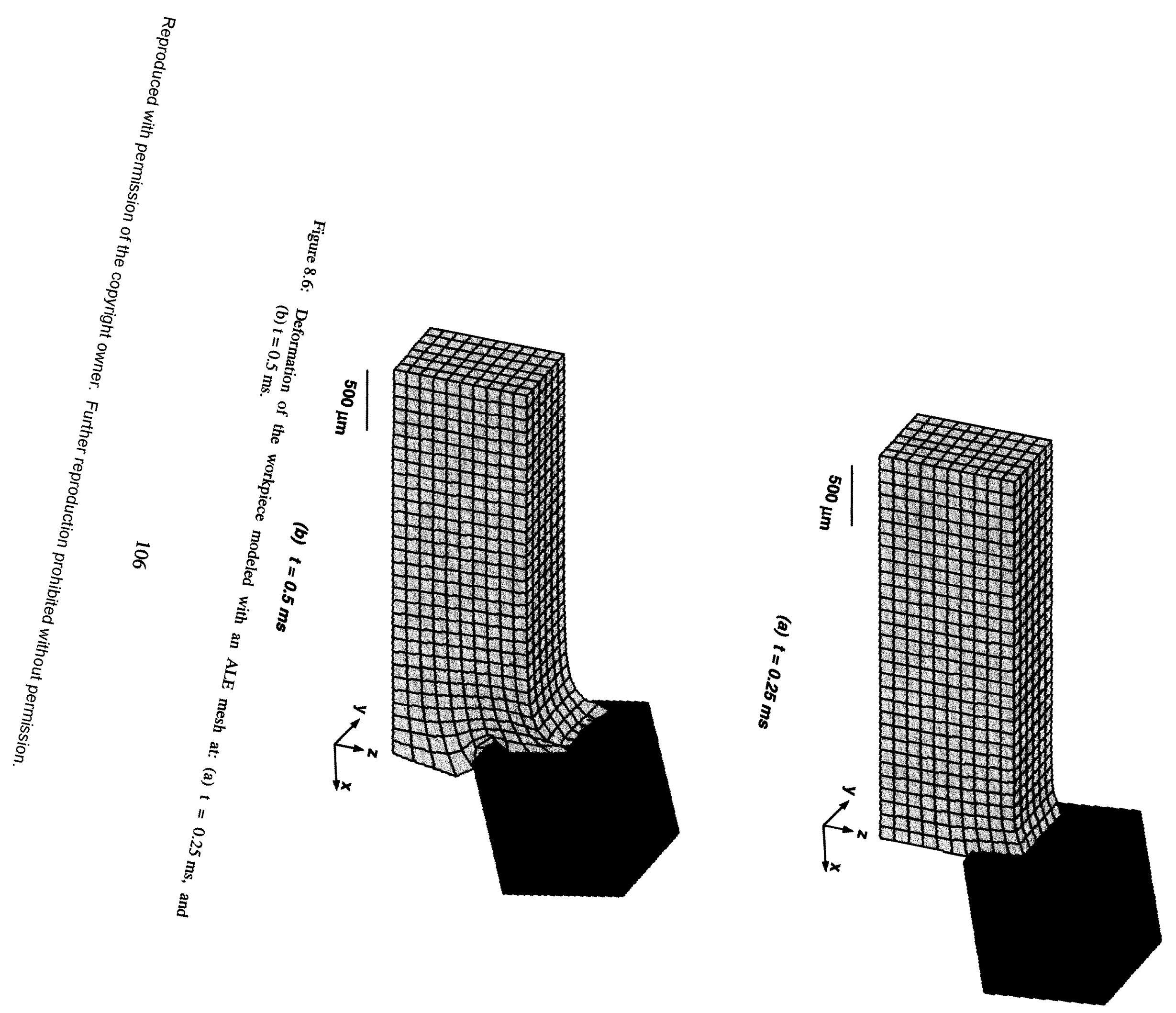




\subsubsection{Strain Distribution}

The strain distribution in the workpiece during incipient chip formation, is shown in Figure 8.7 (a) for a time of $0.25 \mathrm{~ms}$ and Figure 8.7 (b) for a time of $0.5 \mathrm{~ms}$. The maximum strain at $0.25 \mathrm{~ms}$ was 0.50 and occurred adjacent to the tool rake face. Increased strains were concentrated at the top of the workpiece, adjacent to the rake face, and propagated with decreasing magnitude approximately $200 \mu \mathrm{m}$ into the workpiece (in the negative $\mathrm{x}$-direction) and up to $100 \mu \mathrm{m}$ below the cutting line (in the negative z-direction). The maximum strain at a time of $0.5 \mathrm{~ms}$ was 1.47 and also occurred at the rake face of the tool. Strain increase was concentrated along the entire rake face, including the tool tip, and propagated with decreasing magnitude approximately $350 \mu \mathrm{m}$ into the workpiece (in the negative $\mathrm{x}$-direction) and $500 \mu \mathrm{m}$ below the cutting line (in the negative $z$-direction). Since the ALE element formulation was unable to support the formation of a chip under incipient conditions only the SDZ was generated due to friction at the tool-workpiece interface; the PDZ did not form.

\subsubsection{Stress Distribution}

The stress distribution in the workpiece resulting from incipient chip formation studied with the ALE element formulation is shown in Figure 8.8. Similarly to the strain distributions, the stress was analyzed at a time of $0.25 \mathrm{~ms}$, illustrated in Figure 8.8 (a), and at a time of $0.5 \mathrm{~ms}$, illustrated in Figure $8.8(\mathrm{~b})$. The maximum stress at $0.25 \mathrm{~ms}$ was 259.7 $\mathrm{MPa}$ located adjacent to the rake face, which corresponded to the location of the maximum strain. The stresses within the workpiece, decreased with trends similar to those observed in the strain distributions. Stress levels exceeding the yield strength were contained within regions of plastic strain as discussed in Section 8.2.3 (200 $\mu \mathrm{m}$ from the tool-workpiece interface in the negative $\mathrm{x}$-direction and $100 \mu \mathrm{m}$ below the cutting line in the negative $\mathrm{z}$-direction). At $0.5 \mathrm{~ms}$ the maximum stress was $357.1 \mathrm{MPa}$, and occurred along the rake face. Stress values exceeding the yield strength were contained to regions of plastic deformation predicted by the strain distributions, which were $350 \mu \mathrm{m}$ into the workpiece (in the negative $\mathrm{x}$-direction) and $500 \mu \mathrm{m}$ below the cutting line (in the negative z-direction). The stress increase at the tool rake face implied the existence of the SDZ due to tool-workpiece friction. 


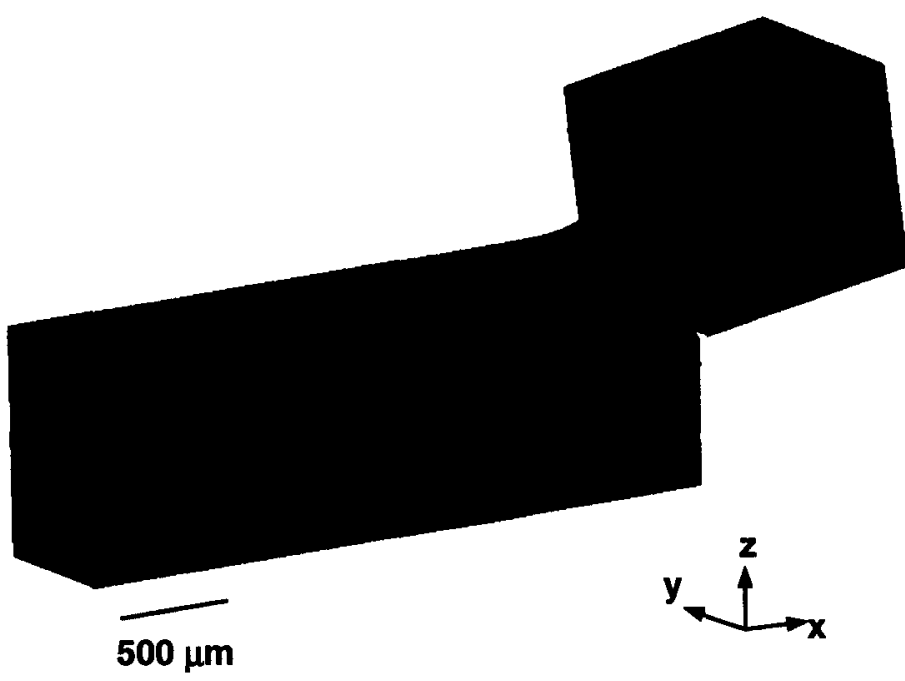

Fringe Levels

$2.000 e+000$

$1.90 B e+000$

$1.800 e+000$

$1.700 e+000$

$1.600 e+000$

$1.500 e+000$

$1.400 \mathrm{e}+000$

(a) $t=0.25 \mathrm{~ms}$

$1.300 e+000$

$1.200 e+000$

$1.100 e+000$

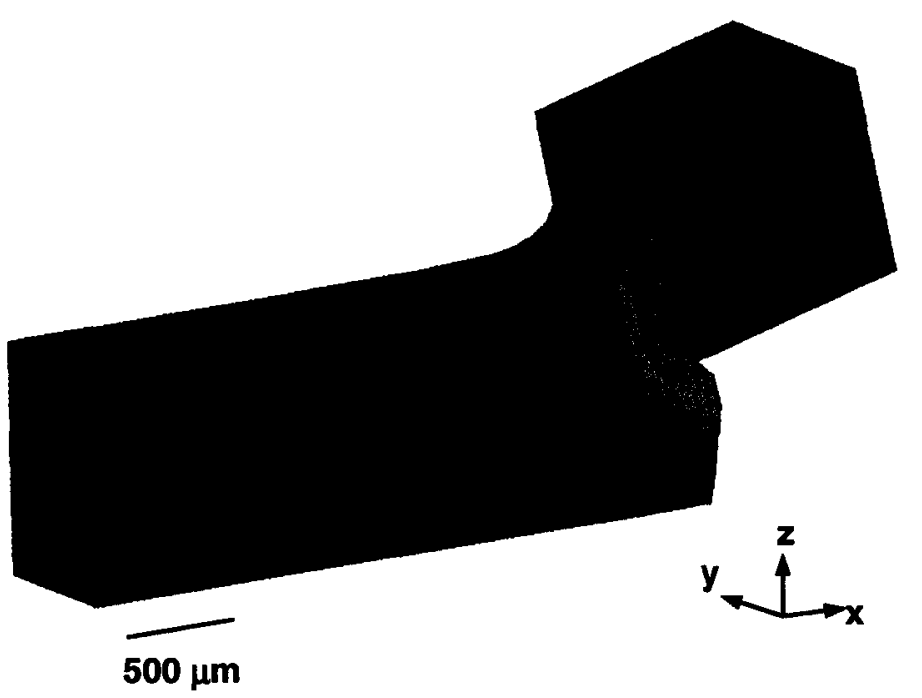

$1.000 e+000$

9.000e-001

8.000e-001

7.000e-001

6.000e-001

5.000e-001

4.000e-001

$3.000 \mathrm{e}-001$

2.000e-001

1.000e-001

$0.000 e+000$

(b) $t=0.5 \mathrm{~ms}$

Figure 8.7: Strain distribution in the workpiece modeled with an ALE element formulation at: (a) $\mathrm{t}=0.25 \mathrm{~ms}$, and ) $\mathrm{t}=0.5 \mathrm{~ms}$. 


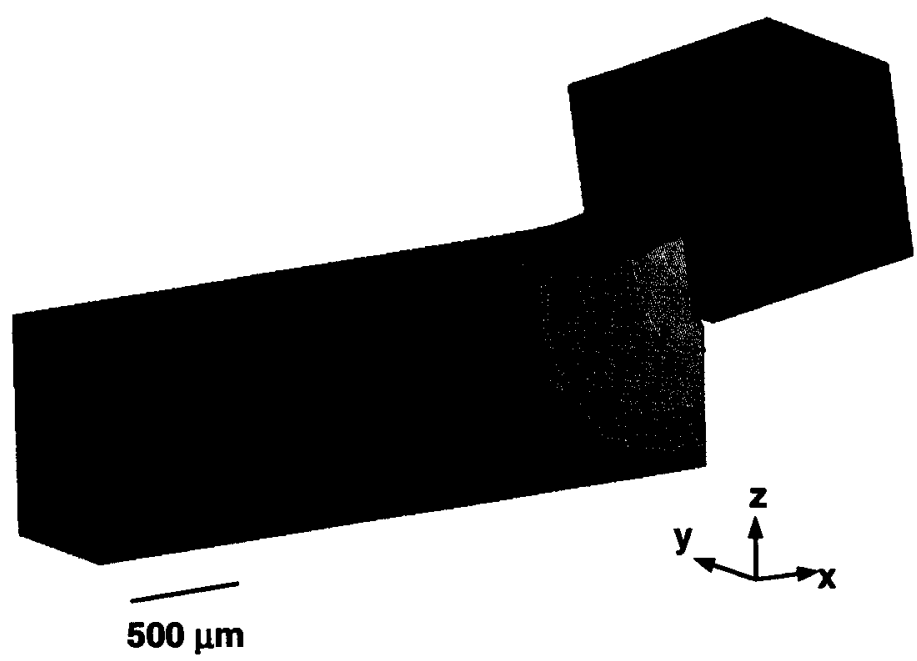

Fringe Levels $4.000 e+002$ $3.800 e+002$ $3.600 e+002$ $3.400 e+002$ $3.200 e+002$ 3.000 e+002

(a) $t=0.25 \mathrm{~ms}$ $2.800 e+002$ $2.600 c+002$ $2.400 e+002$ $2.200 e+002$ $2.000 e+002$

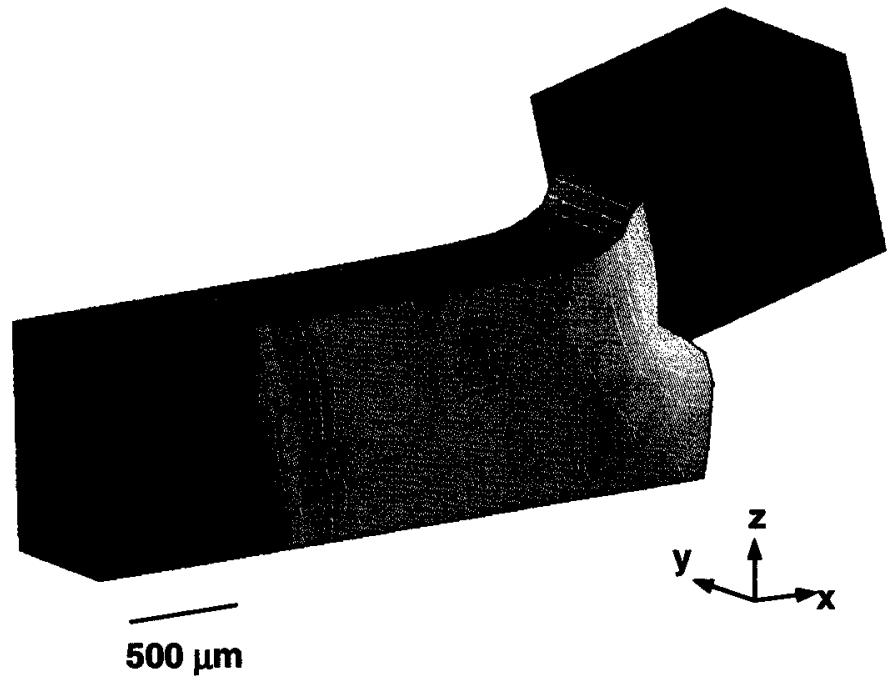
$1.600 e+002$ $1.600 \mathrm{e}+002$ $1.400 e+002$ $1.200 e+002$ $1.000 c+002$ $8.000 e+001$ 6.000e+001 4.000e+001 $2.000 e+001$ $0.000 e+000$

(b) $t=0.5 \mathrm{~ms}$

Figure 8.8: Distribution of stress in units of MPa, for the workpiece modeled with an ALE element formulation at: (a) $\mathrm{t}=0.25 \mathrm{~ms}$, and (b) $\mathrm{t}=0.5 \mathrm{~ms}$. 


\section{CHAPTER 9}

\section{Finite Element Results III :}

\section{Metal Cutting Model Employing the Eulerian Element Formulation}

The investigations of metal cutting modeled utilizing the Eulerian element formulation, were performed according to procedures described in Chapter 6 . The analyses were completed on three different material models, and two different geometries: one with a single plane of symmetry and the other with two planes of symmetry. Simulations were performed using the FE code LS-DYNA 970 on dual Athlon $1.8 \mathrm{GHz}$ processors with one gigabyte of memory.

\subsection{EFFECT OF USING ONE PLANE AND TWO PLANES OF SYMMETRY}

The two geometries used to model the metal cutting process were discussed in Section 6.1.1 and were illustrated in Figure 6.1. The model with one plane of symmetry modeled $1.5 \mathrm{~mm}$ (or one half) of the actual width of the workpiece, and was composed of 188,436 elements. The second model utilized two planes of symmetry, modeled $0.27 \mathrm{~mm}$ (or less than 10\%) of the workpiece width, and contained 16,746 elements. The same material model, Material Model 1, was employed in simulating metal cutting using both geometries; input parameters were kept constant, including rake angle (negative 5 degrees), clearance angle ( 8 degrees), feed $(0.25 \mathrm{~mm})$, and applied velocity $\left(600 \mathrm{~mm} \cdot \mathrm{s}^{-1}\right)$. The processing time was approximately 670 hours (or 28 days) for the larger model, and approximately 127 hours (or 5 days) for the reduced geometry. In order to ensure that reducing the geometry, by adding a second plane of symmetry, would not diminish accuracy, the results for both of the simulations were compared on the basis of geometry (Section 9.1.1), strain (Section 9.1.2), and stress (Section 9.1.3). All investigations were performed along the workpiece cross-section, which lay on the single plane of symmetry in the larger geometry and on either of the two planes of symmetry in the reduced geometry. Comparisons were made at times of $7.5 \mathrm{~ms}, 10.0 \mathrm{~ms}$, and $15.0 \mathrm{~ms}$. 
Once it was confirmed that the reduced geometry could replicate the results of the larger geometry, two planes of symmetry were used for all simulations thereafter.

\subsubsection{Deformation of the Workpiece}

The comparison between the chip formation for each of the geometrical models used is shown in Figure 9.1 (a three-dimensional view) and in Figure 9.2 (a cross-sectional view). At $7.5 \mathrm{~ms}$, the chip had just begun to form and travel along the rake face. Curling of the chip was evident at a time of $10.0 \mathrm{~ms}$, and a completely curled chip was approaching the workpiece surface at a time of $15.0 \mathrm{~ms}$. For all three assessment times, the chip formation matched very well between one and two symmetry plane(s). The steady state chip thickness of approximately $0.67 \mathrm{~mm}$ was similar in both sets of analyses. Slight, yet negligible, discrepancies were noted in the chip outline, but were likely the cause of averaging deviations.

\subsubsection{Strain Distribution}

The strain distributions in both of the geometries are shown in Figure 9.3, at $7.5 \mathrm{~ms}, 10.0 \mathrm{~ms}$, and $15.0 \mathrm{~ms}$. The maximum strain occurred at the tool tip for all considered times, with a maximum strain of 5.0 for $7.5 \mathrm{~ms}, 5.4$ for $10.0 \mathrm{~ms}$, and 5.5 for $20.0 \mathrm{~ms}$. The extent of plastic deformation under the machined surface was constant in time and approximately $300 \mu \mathrm{m}$ below the cutting line for geometries with one and two symmetry plane(s). The distribution of strain along the PDZ remained the same through time, with the maximum strain at the tool tip decreasing to a minimal strain (of approximately 0.5 ) at the chip root. All strain distributions illustrated an increase in strain at the tool rake face within the SDZ. At times greater then $10.0 \mathrm{~ms}$ a strain increase was also apparent along the rake face at $400 \mu \mathrm{m}$ above the tool tip. The strain decreased within the SDZ from a high at the rake face to a steady value of strain between 1.5 and 2.0 in the chip. The geometry utilizing two symmetry planes has strain predictive capabilities equal to those of the one symmetry plane geometry within the machined surface, SDZ, and PDZ. The distribution of strain within the formed chip varies slightly between the two geometries; however, this is not a region of interest for this study, and the differences were not considered significant. 
One plane of symmetry

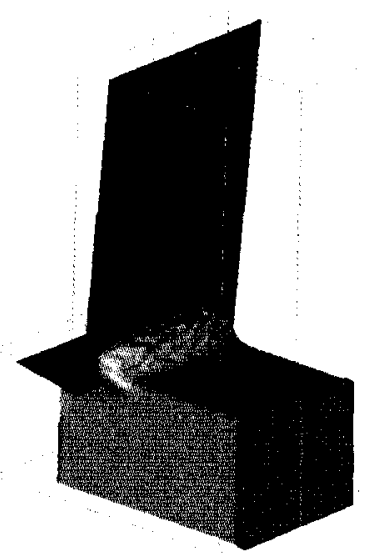

(a) $t=7.5 \mathrm{~ms}$

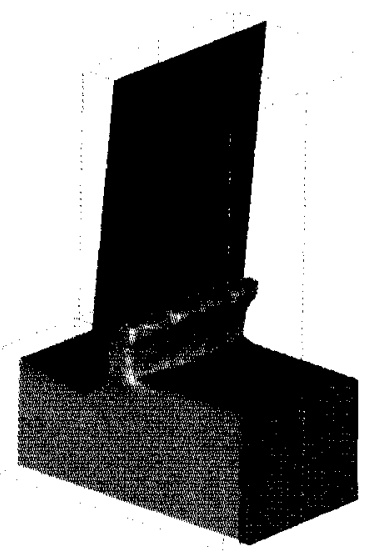

(b) $t=10.0 \mathrm{~ms}$

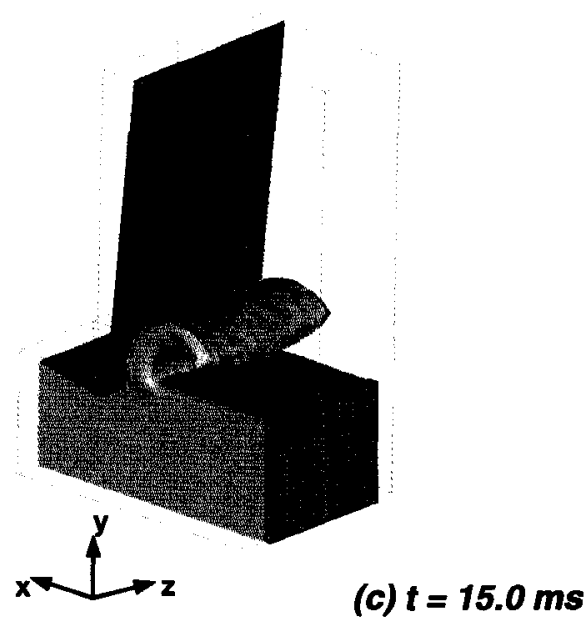

Two planes of symmetry
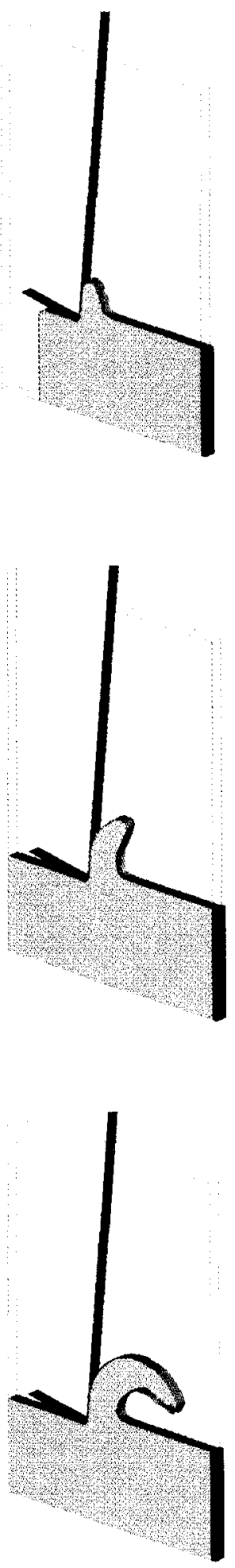

Figure 9.1: Chip formation for models with one and two plane(s) of symmetry at times of: (a) $7.5 \mathrm{~ms}$, (b) $10.0 \mathrm{~ms}$, and (c) $15.0 \mathrm{~ms}$. 

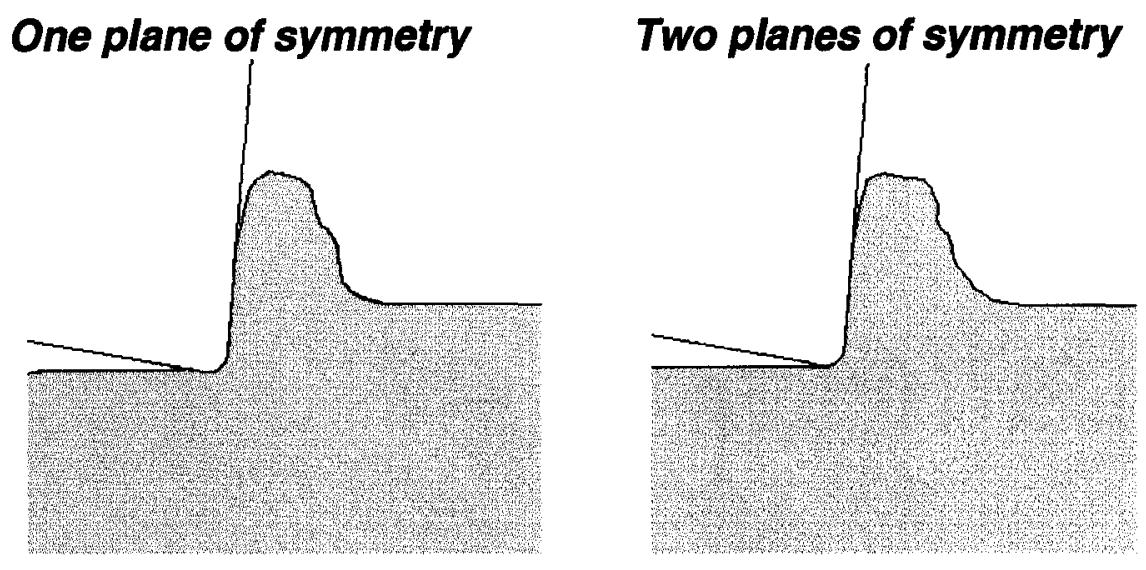

$500 \mathrm{um}$

(a) $t=7.5 \mathrm{~ms}$
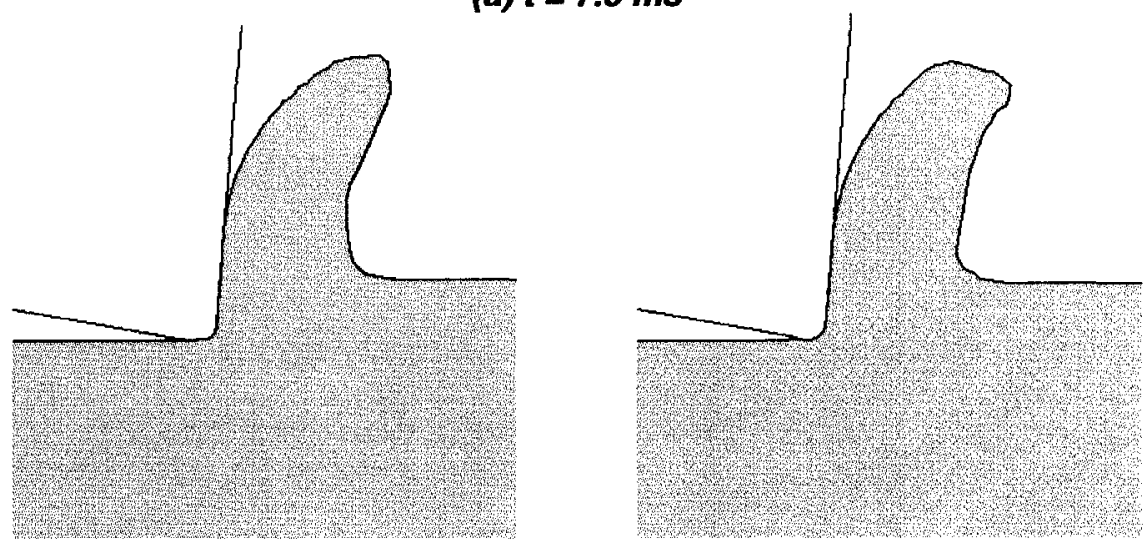

$500 \mathrm{um}$

(b) $t=10.0 \mathrm{~ms}$

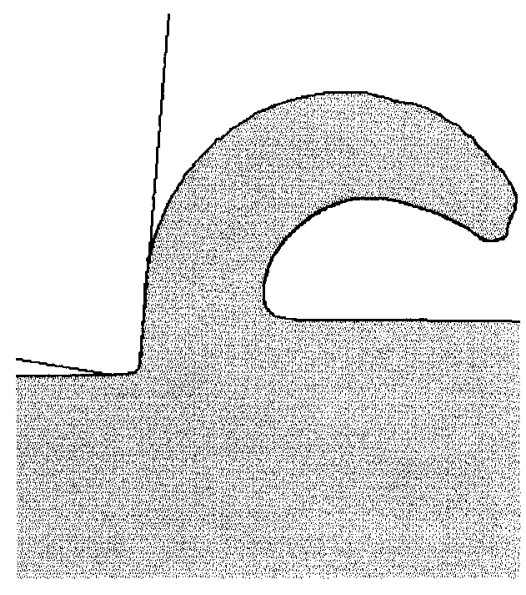

$500 \mathrm{um}$

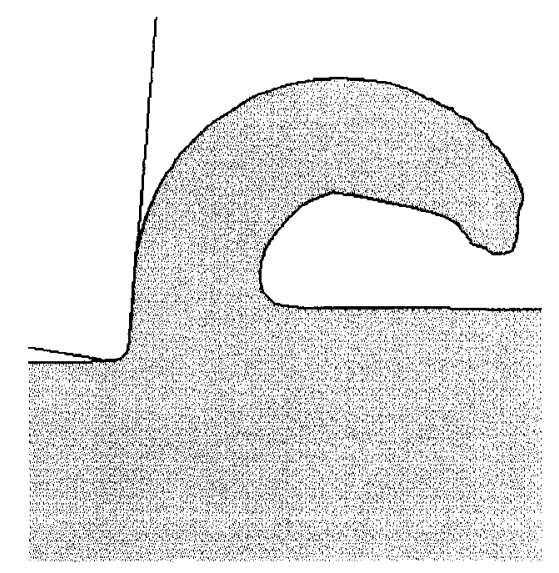

(c) $t=15.0 \mathrm{~ms}$

Figure 9.2: Comparison of cross-sectional chip geometry generated by models with one and two plane(s) of symmetry, at times of: (a) $7.5 \mathrm{~ms}$, (b) $10.0 \mathrm{~ms}$, and (c) $15.0 \mathrm{~ms}$. 


\section{One plane of symmetry}

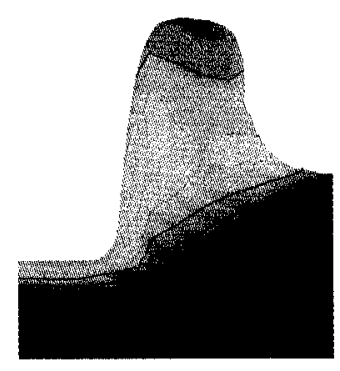

$500 \mu \mathrm{m}$

(a) $t=7.5 \mathrm{~ms}$

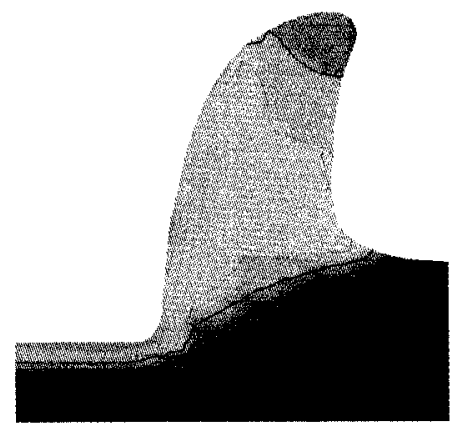

$500 \mu \mathrm{m}$
Two planes of symmetry

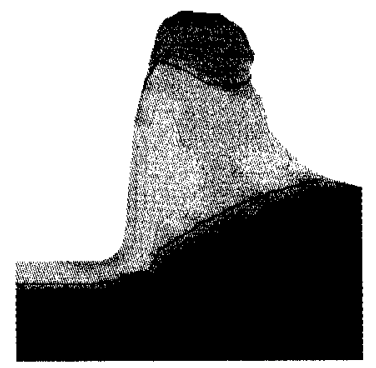

Fringe Levels

$0.000 e+000$

7.600 e+000

$7.200 e+000$

$6.800 e+000$

6.400e+000

$6.000 \mathrm{e}+000$

$5.600 \mathrm{e}+000$

$5.200 e+000$

4.800e+000

4.400e+000

4.000e+000

$3.600 e+000$

3.200 +000

2.000e+000

$2.400 e+000$

2.000e+000

1.600 e+000

1.200 e+000

0.000e-001

4.000e-001

D.000e+000
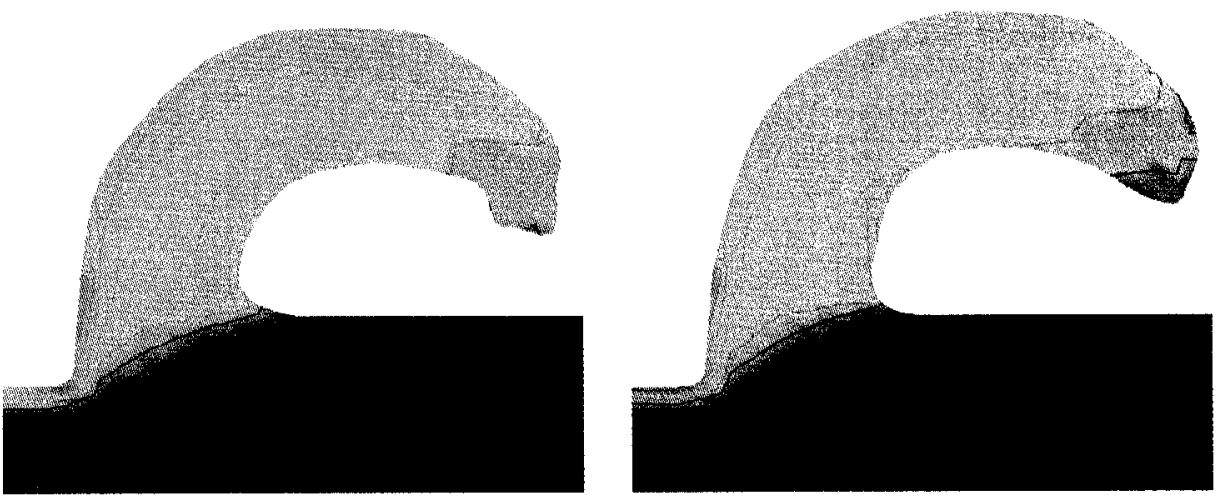

$500 \mu \mathrm{m}$

(c) $t=15.0 \mathrm{~ms}$

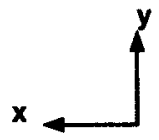

Figure 9.3: Comparison of strain distributions in models with one and two plane(s) of symmetry, at times of: (a) $7.5 \mathrm{~ms}$, (b) $10.0 \mathrm{~ms}$, and (c) $15.0 \mathrm{~ms}$. 


\subsubsection{Stress Distribution}

The distribution of stress for both studied geometries and each of the considered times is illustrated in Figure 9.4. For each, a maximum stress of $340 \mathrm{MPa}$ occurred at the tool tip. The concentration of increased stresses, in the range of 320 to $330 \mathrm{MPa}$, spanned between the tool tip and the chip root defining the PDZ. The width of the PDZ was defined on the basis of the stress concentration and was approximated to range between 150 and $200 \mu \mathrm{m}$. The extent of plastic deformation below the tool tip was approximately $300 \mu \mathrm{m}$ for all stress distributions considered, which was consistent with observations made from strain distributions. The most noticeable discrepancies between the stress distributions of the considered geometries occurred within the chip and undeformed region of the workpiece, which were not the areas under close scrutiny when correlating the results. The most important regions, including the PDZ, the SDZ, and the machined surface, generally showed good agreement in both stress levels and their distributions. 


\section{One plane of symmetry}

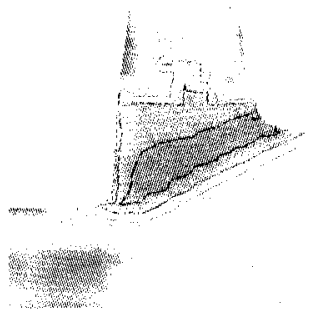

$500 \mu \mathrm{m}$

(a) $t=7.5 \mathrm{~ms}$

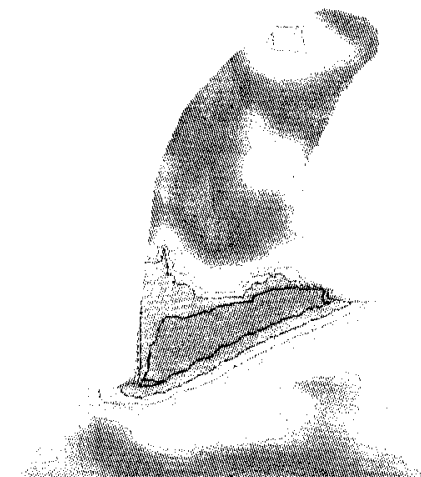

$500 \mu \mathrm{m}$

\section{(b) $t=10.0 \mathrm{~ms}$}

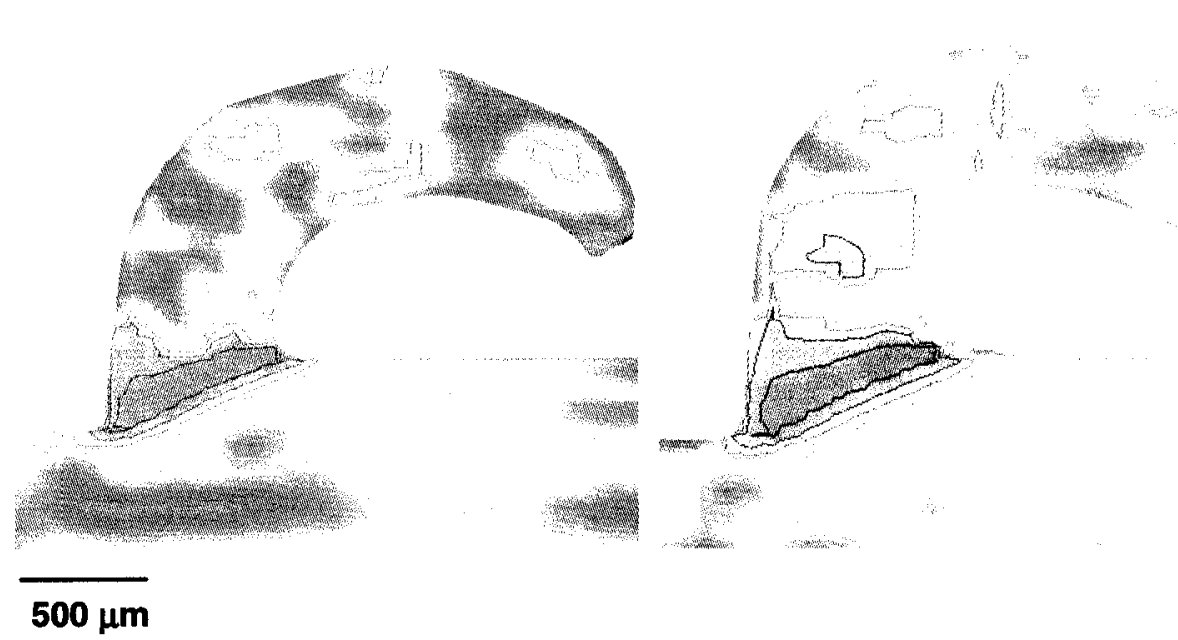

(c) $t=15.0 \mathrm{~ms}$
Fringe Levels

$3.400 e+005$

$3.230 e+005$

$3.060 e+005$

$2.890 e+005$

$2.720 e+005$

$2.550 e+005$

$2.380 e+005$

$2.210 \mathrm{e}+005$

$2.040 e+005$

$1.870 e+005$

$1.700 e+005$

$1.530 e+005$

$1.360 \mathrm{e}+005$

$1.190 e+005$

$1.020 e+005$

8.500e+004

6.800e+004

$5.100 e+004$

$3.400 e+004$

$1.700 e+004$

$0.000 e+000$

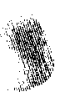

Figure 9.4: Comparison of stress distributions in units of $\mathrm{kPa}$, for models with one and two plane(s) of symmetry, at times of: (a) $7.5 \mathrm{~ms}$, (b) $10.0 \mathrm{~ms}$, and (c) $15.0 \mathrm{~ms}$. 


\subsection{RESULTS GENERATED FROM MATERIAL MODEL 1}

Material Model 1 was based on the Johnson-Cook constitutive equation, with constants obtained from curve fitting the stress-strain relationship to the experimental results, as discussed in Section 6.1.3.1. The interaction between the workpiece and tool was modeled with several different coefficients of friction varying between 0.0 and 0.9 , with processing time varying from 125 hours and 18 minutes to 137 hours and 25 minutes.

\subsubsection{Energy Balance}

An energy balance typical of simulations performed with Material Model 1 is shown in Figure 9.5. It is evident from this figure that total energy consists mostly of internal energy, since they are approximately equivalent. Since the energy required to deform the workpiece material (internal energy) is so much greater in scale than the kinetic energy produced by the workpiece velocity, the kinetic energy appears to be zero; but in fact it is only small in comparison. It was extremely difficult to control penetration, and therefore sliding energy was detected but in very small amounts. Ideally work done should be completely converted into energy, and external work and total energy should equal; however, they deviated above a time of $10.0 \mathrm{~ms}$. The time of $10.0 \mathrm{~ms}$ corresponded to the instant when the workpiece left the airmesh after having travelled along the entire model width in the positive x-direction. Energy is calculated at the end of every time step with the deformed material being the source of internal and kinetic energy; therefore, the outgoing deformed material possessed kinetic energy and stored internal energy that was no longer included in the total energy. External work is the sum the work done in all time steps required to displace the material and is cumulative from the beginning to the end of the simulation; hence, the work required to acquire these internal and kinetic energies was still included in the cumulative external work causing the external work to exceed the total energy. 


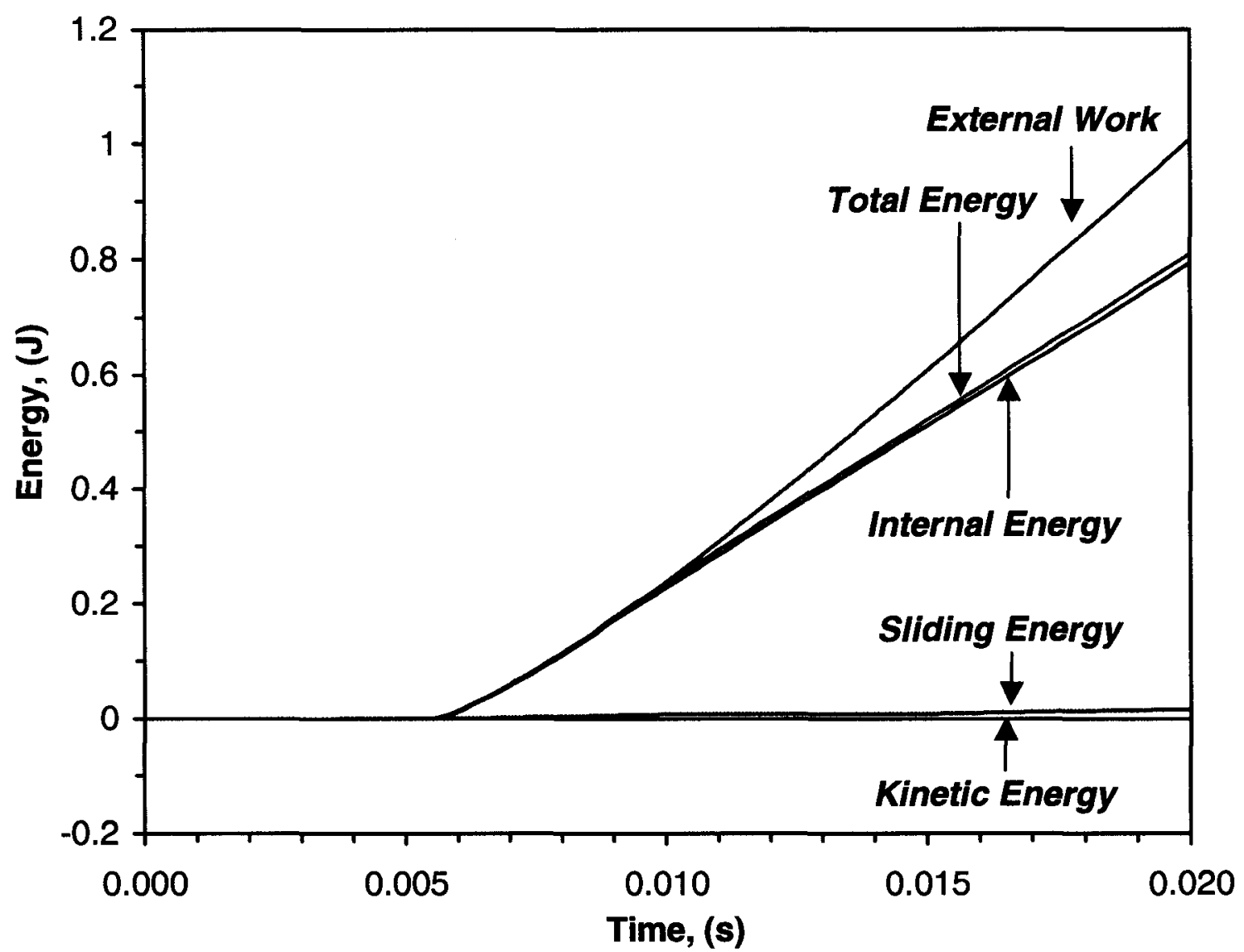

Figure 9.5: Energy balance typical of the metal cutting simulated in the Eulerian element formulation study, with workpiece modeled as Material Model 1. 


\subsubsection{Deformation of the Workpiece}

The friction between the tool and workpiece has a large influence on the workpiece deformation. As discussed in Section 6.1.5, the Eulerian-Lagrangian contact is limited to defining a constant coefficient of friction, making it difficult to approximate a constant value to replace the variable friction along the tool-chip contact length. Numerical simulations were completed using different values of the coefficient of friction to investigate the relationship between chip thickness and the coefficient of friction for Material Model 1. The investigation studied friction coefficients of $0.0,0.2,0.4,0.6,0.8$ and 0.9, and the results of the simulations are summarized in Figure 9.6 (a). The relationship between the coefficient of friction $(\mu)$ and the chip thickness $\left(t_{c}\right)$ is plotted in Figure $9.6(\mathrm{~b})$, and it obeys the following quadratic equation:

$$
t_{c}=0.7112 \mu^{2}+0.6287 \mu+0.6678 .
$$

Figure 9.6 (b) paired with Equation (9-1), showed that a coefficient of friction of 0.84 best replicated the experimental chip thickness of $1.7 \mathrm{~mm}$ observed in Figure 2.17. Ernst and Merchant [70] observed similar increase in chip thickness with progression from a lubricated to dry machining process, which establishes the capability of this FE model to simulate all machining conditions from fully lubricated to dry. Consequently, another simulation was completed employing a coefficient of friction equal to $\mu=0.84$. The geometry of the chip formed during this simulation was in excellent agreement with the experimental chip thickness of $1.7 \mathrm{~mm}$.

\subsubsection{Strain Distribution}

The strain distribution in the material ahead of the tool tip, modeled with Material Model 1 and $\mu=0.84$, is shown in Figure 9.7. The plastic strain within the material varied between 0.0 , where no plastic deformation occurred, and 7.5 at $400 \mu \mathrm{m}$ above the tool tip, which was the maximum plastic strain accumulated in the material. The strain distribution showed an increase in strain below the machined surface and adjacent to the tool rake face, due to the material displacement at the tool tip and the friction at the chip-tool interface. Maximum strain within the machined surface was 3.6 and occurred in the proximity of the cutting line. The strain in the machined surface decreased to approximately 0.025 at a depth of $300 \mu \mathrm{m}$ beneath the cutting line, and all traces of 


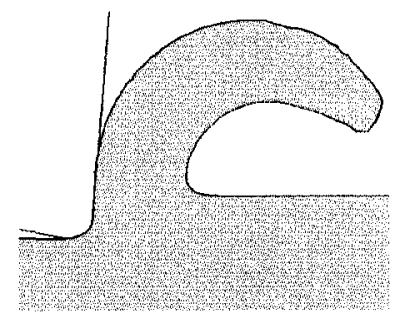

$\mu=0.0, t_{c}=0.67$

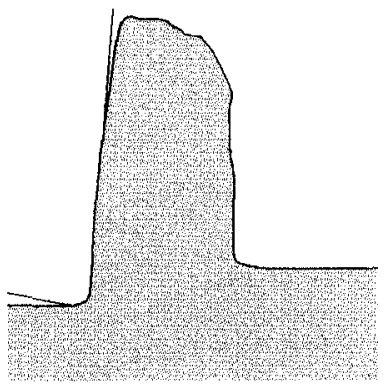

$\mu=0.6, t_{c}=1.27$

$500 \mu \mathrm{m}$

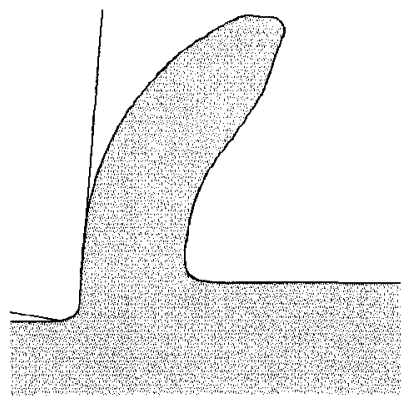

$\mu=0.2, t_{c}=0.81$

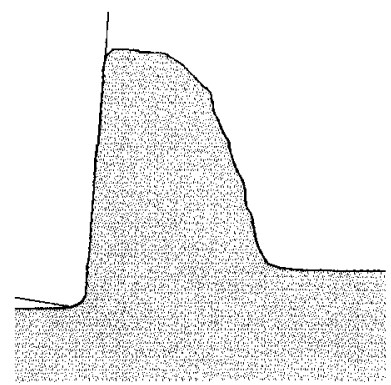

$\mu=0.8, t_{c}=1.65$

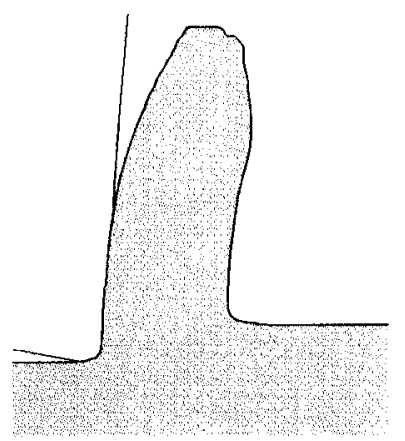

$\mu=0.4, t_{c}=1.06$

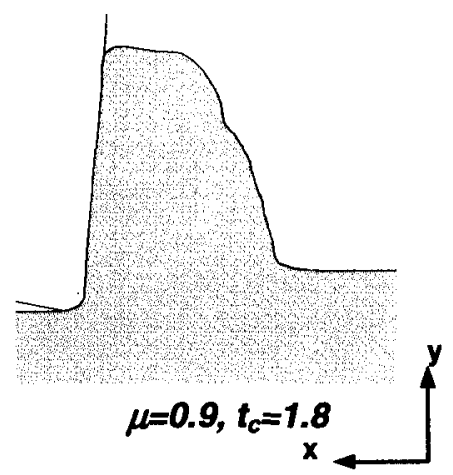

(a)

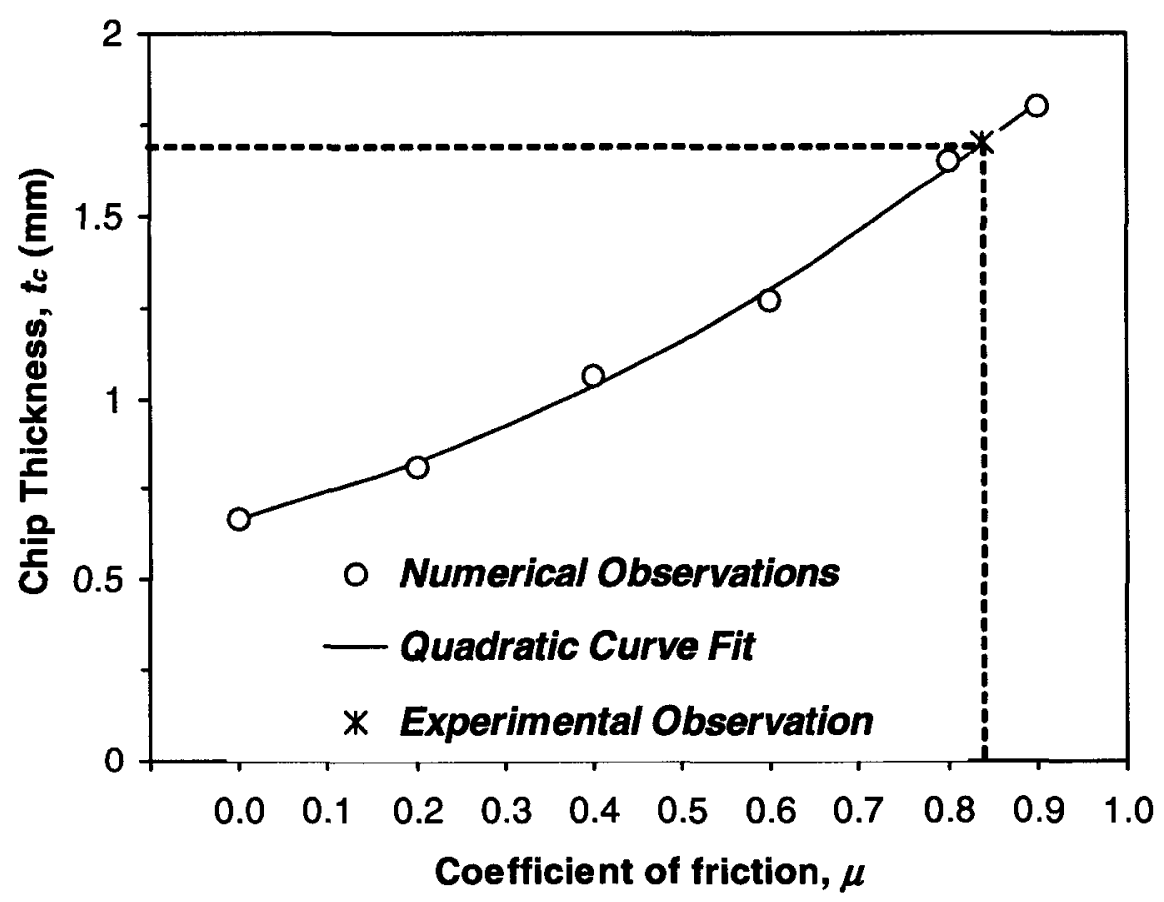

(b)

Figure 9.6: Investigation of friction coefficient effect on chip thickness for Material Model 1, shown are: (a) chip geometry with corresponding coefficients of friction, and (b) coefficient of friction vs. chip thickness plot, with experimental data noted. 


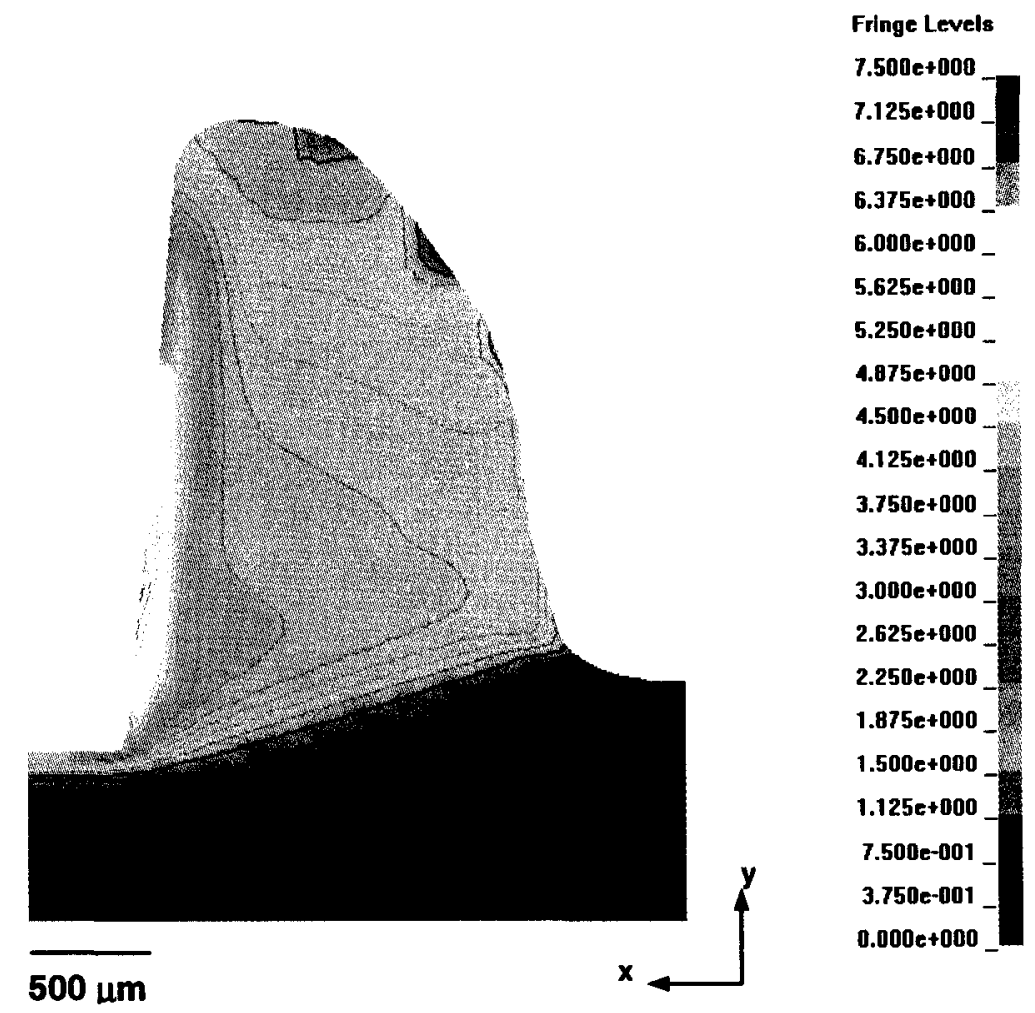

Figure 9.7: Strain distribution in the material ahead of the tool tip in the Eulerian element formulation study, with workpiece modeled as Material Model 1 and $\mu=0.84$.

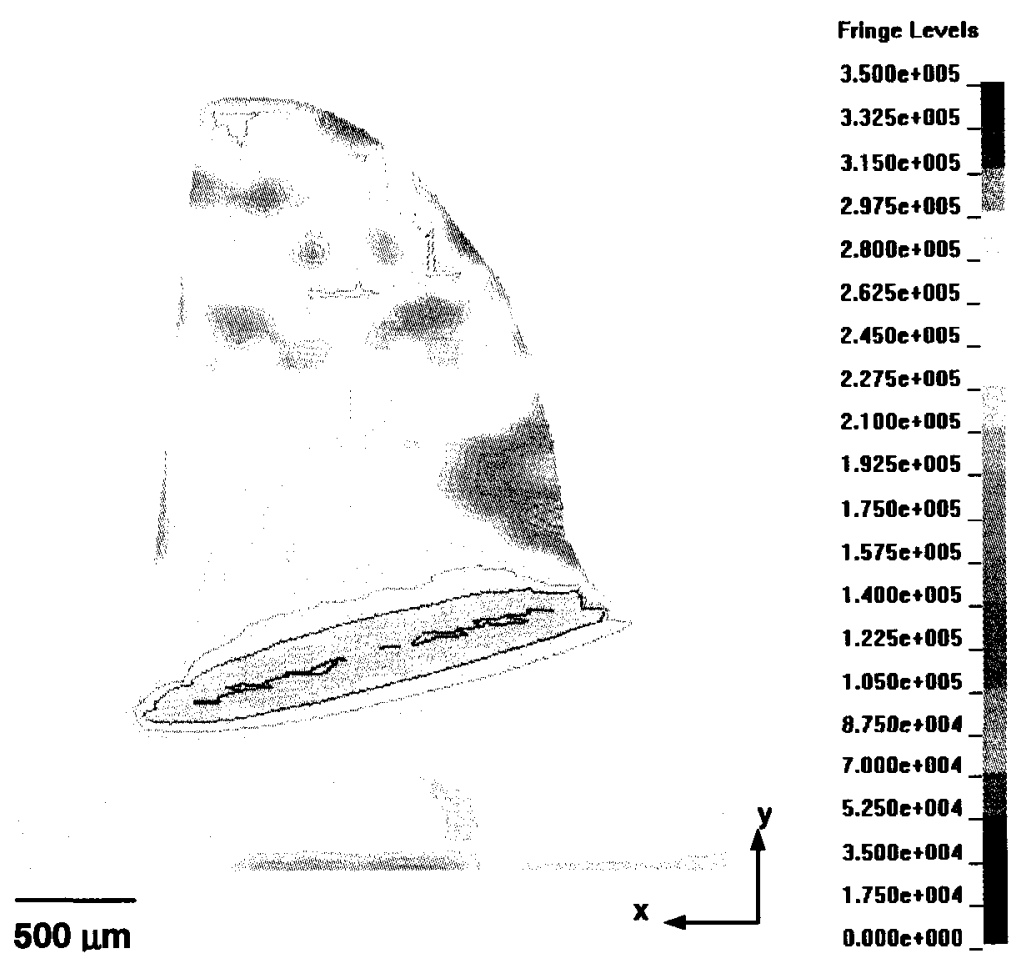

Figure 9.8: Stress distribution in the material ahead of the tool tip, in units of $\mathrm{kPa}$, in the Eulerian element formulation study, with workpiece modeled as Material Model 1 and $\mu=0.84$. 
plastic strain vanished beyond depths of $350 \mu \mathrm{m}$ beneath the cutting line. Severe deformation within the SDZ was contained to $100 \mu \mathrm{m}$ within the tool rake face. The strain decreased as the distance from the rake face increased. The strain within the chip ranged between 1.5 and 2.1. A maximum strain within the PDZ of 4.5 occurred immediately ahead of the tool tip and decreased along the primary shear plane to a minimum strain of 0.1 at the chip root.

\subsubsection{Stress Distribution}

The computed values of von Mises stress distribution, corresponding to Material Model 1 and $\mu=0.84$, are presented in Figure 9.8. At termination time, the von Mises stress in the workpiece reached a maximum value of $342 \mathrm{MPa}$ at the tool tip. The plastic deformation zone beneath the machined surface was estimated to extend to $350 \mu \mathrm{m}$ below the cutting line in strain observations, which was in agreement with the stress distributions. Within the machined surface, a maximum local stress of $265 \mathrm{MPa}$ occurred adjacent to the cutting line and decreased to the vicinity of yield stress levels at depths exceeding the region of plastic deformation. Concentration of high stresses was observed in the PDZ which contained stress levels ranging from 300 to $330 \mathrm{MPa}$ and approximated the width of the zone as $600 \mu \mathrm{m}$. The stress decreased above the PDZ, within the formed chip, and below the PDZ into the undeformed material. The stress distribution within the chip did not indicate an existence of a SDZ, since no stress increase occurred adjacent to the rake face of the tool.

\subsubsection{External Work}

Work done during workpiece deformation was tracked with respect to time and the applied friction coefficient, and is presented in Figure 9.9 as external work per unit depth of the model. Since external work is the work required to deform the material, it was equal to zero until the workpiece contacted the tool at a time of $5.5 \mathrm{~ms}$. As the workpiece advanced, an increasing amount of work was required to continually form the chip, and thus external work increased linearly with respect to time. The highest external work was observed at the termination time of $20.0 \mathrm{~ms}$. When considering the variation of external work with respect to the friction coefficient, the required work increased 


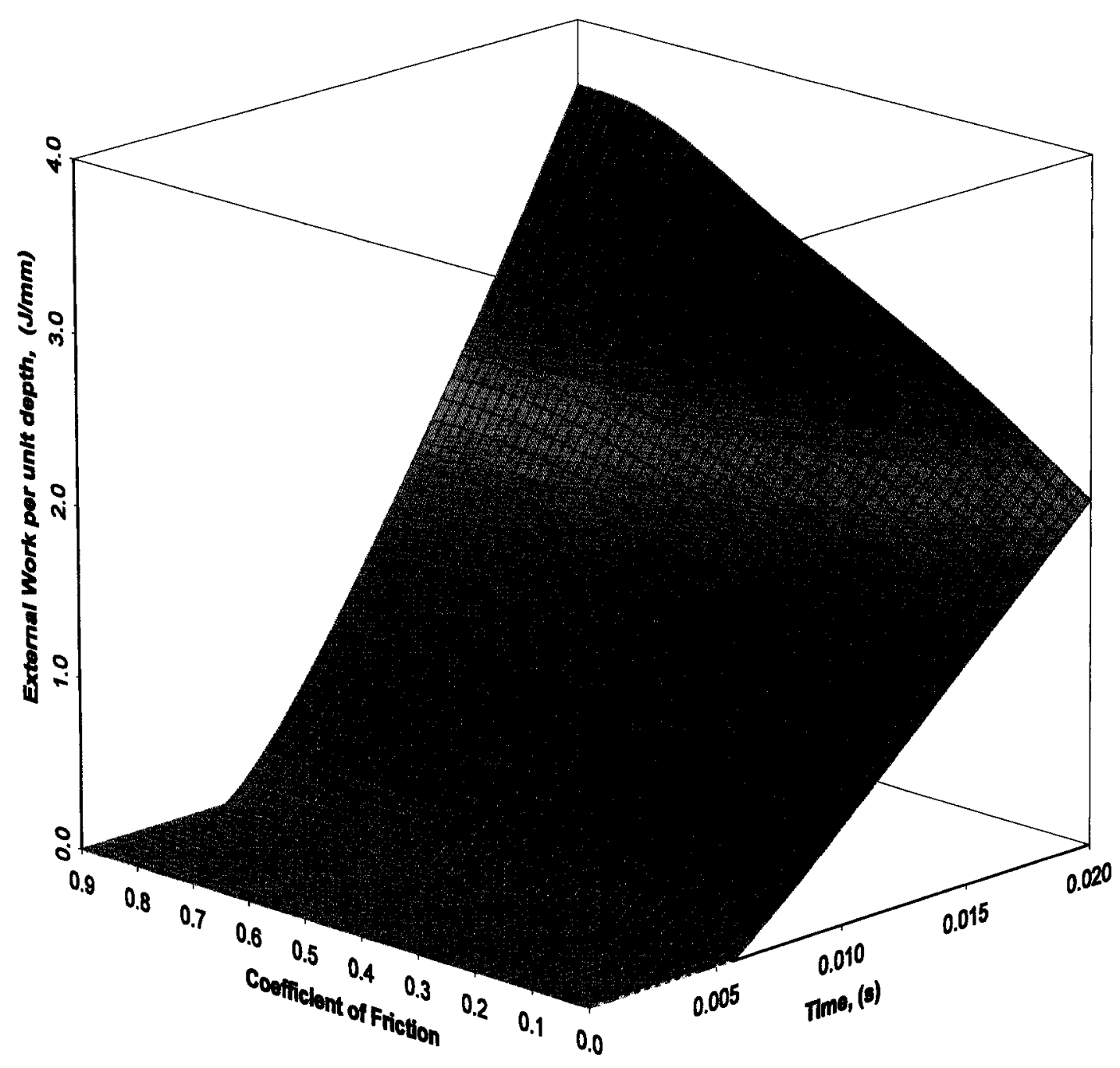

Figure 9.9: Surface plot of external work variation, in units of $\mathrm{J} \cdot \mathrm{mm}^{-1}$, with respect to time and friction, in the Eulerian element formulation study with workpiece modeled as Material Model 1. 
with higher coefficients of friction. Increasing friction provided additional resistance along the tool-workpiece interface, and therefore more work was required to form a chip. The external work at termination varied from $2.01 \mathrm{~J} \cdot \mathrm{mm}^{-1}$ for $\mu=0.0$ to $3.62 \mathrm{~J} \cdot \mathrm{mm}^{-1}$ for $\mu=0.9$. For the friction coefficient of $\mu=0.84$, which best reproduced the experimental chip geometry, the external work increased linearly from 0.0 , at a time of $5.5 \mathrm{~ms}$, to a maximum of $3.60 \mathrm{~J} \cdot \mathrm{mm}^{-1}$ at the time of termination.

\subsubsection{Force and Power Predictions}

The forces derived from the interaction between the Lagrangian tool and Eulerian workpiece were analyzed with respect to time and the applied coefficient of friction. Considering the model orientation shown in Figure 6.2 and the force equilibrium in Figure 2.4, the numerical $\mathrm{x}$-component of the resultant contact force corresponded to the cutting force $\left(F_{c}\right)$, the y-component corresponded to the thrust force $\left(F_{t}\right)$, and the z-component corresponded to the radial force $\left(F_{r}\right)$. Figures 9.10 and 9.11 are variations of cutting and thrust forces, respectively, with respect to time and friction. The values of force obtained numerically were multiplied by a factor of 11.1 in order to account for the experimental contact surface spanning along a $3.0 \mathrm{~mm}$ wall thickness, as opposed to the model depth of $0.27 \mathrm{~mm}$. The radial force, was negligible in comparison to cutting and thrust forces, which is in agreement with assumptions made by Piispanen [8] listed in Section 2.1.5. Both the cutting and thrust forces remained zero until contact between the workpiece and tool occurred, which was at $5.5 \mathrm{~ms}$. Beyond initial contact, cutting and thrust forces rose rapidly with time and eventually reached a steady state value. The higher the coefficient of friction between the tool and workpiece, the longer it took for a chip to reach steady state formation and for the forces to reach steady state values. The thrust force was significantly lower than the cutting force, for each coefficient of friction, throughout simulation time. Both the cutting and thrust forces were found to increase with respect to increasing friction coefficient applied between the tool and workpiece. The steady state value of the thrust force increased from $211 \mathrm{~N}$ for $\mu=0.0$ to $1030 \mathrm{~N}$ for $\mu=0.9$, which corresponds to a $400 \%$ increase. The steady state cutting forces, on the other hand, increased from $738 \mathrm{~N}$ for $\mu=0.0$ to $1520 \mathrm{~N}$ for $\mu=0.9$, which corresponds to a $100 \%$ increase. This result indicated that increasing the coefficient of friction between 
the tool and workpiece has a larger impact on the thrust force than on the cutting force. For $\mu=0.84$, the cutting and thrust forces at termination time were $1430 \mathrm{~N}$ and $962 \mathrm{~N}$, respectively.

Utilizing Equation (2-10), the surface plot in Figure 9.12 was generated from the cutting forces presented in Figure 9.10 and summarizes the variation of power required for the cutting operations simulated. The power follows the same trends as the cutting forces in Figure 9.10. The maximum power consumption was $442 \mathrm{~W}$ for $\mu=0.0$, and $910 \mathrm{~W}$ for $\mu=0.9$. Figure 9.12 illustrates that an increase in power consumption for the metal cutting process occurs as the friction between the tool and workpiece increases. 


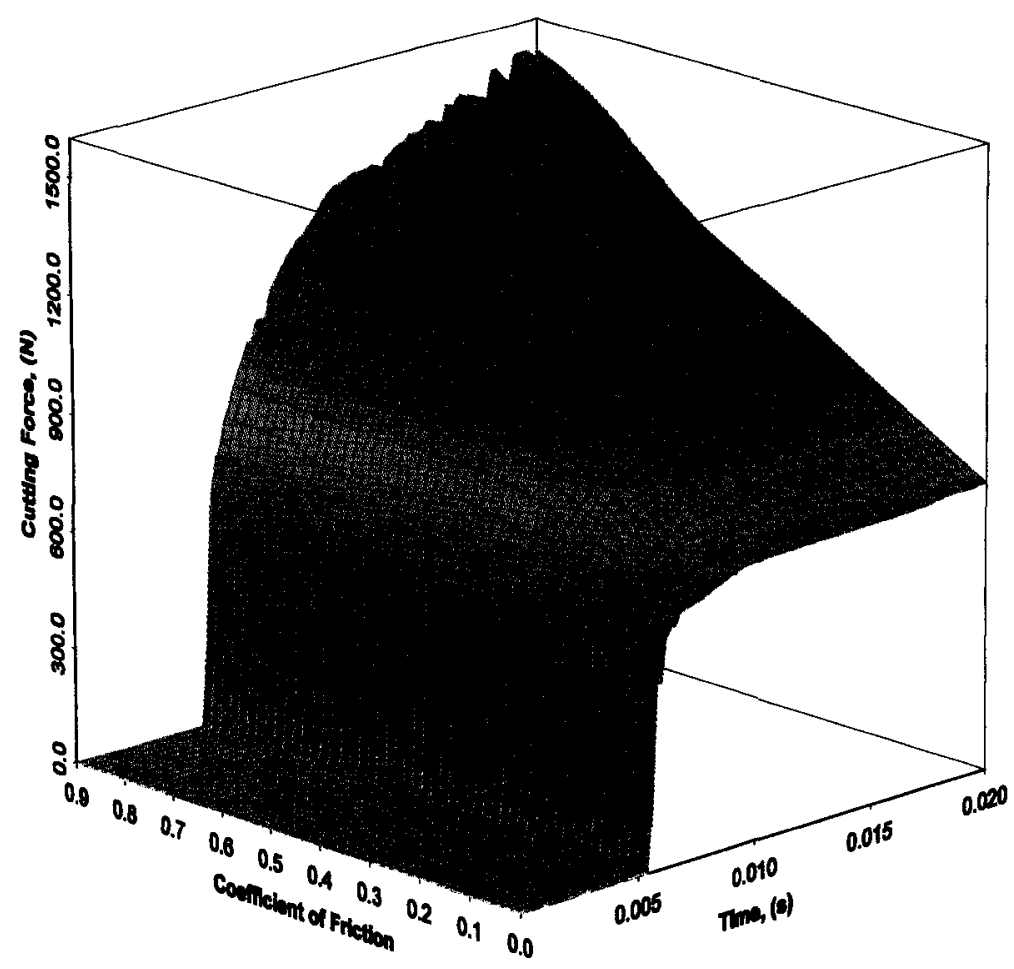

Figure 9.10: Surface plot of cutting force $\left(F_{c}\right)$ variation in units of $\mathrm{N}$, with respect to time and friction, in the Eulerian element formulation study with workpiece modeled as Material Model 1.

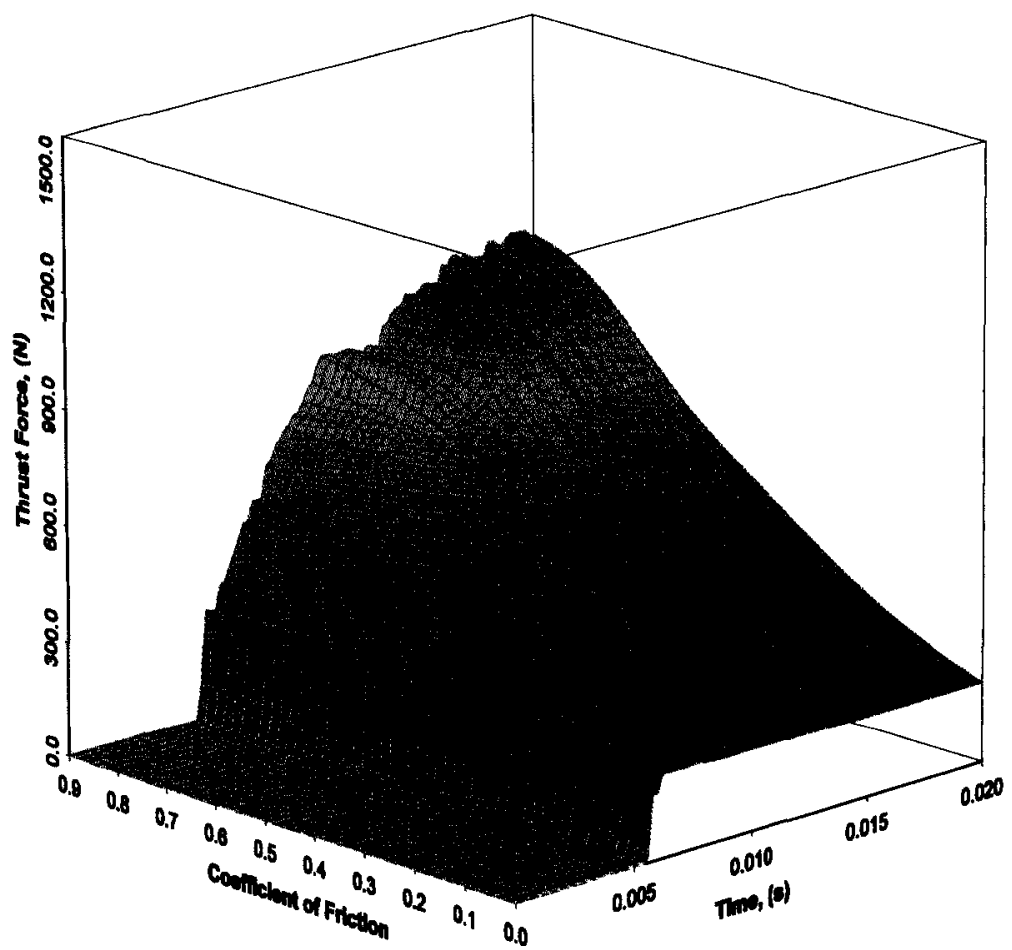

Figure 9.11: Surface plot of the thrust force $\left(F_{t}\right)$ variation in units of $\mathrm{N}$, with respect to time and friction, in the Eulerian element formulation study with workpiece modeled as Material Model 1. 


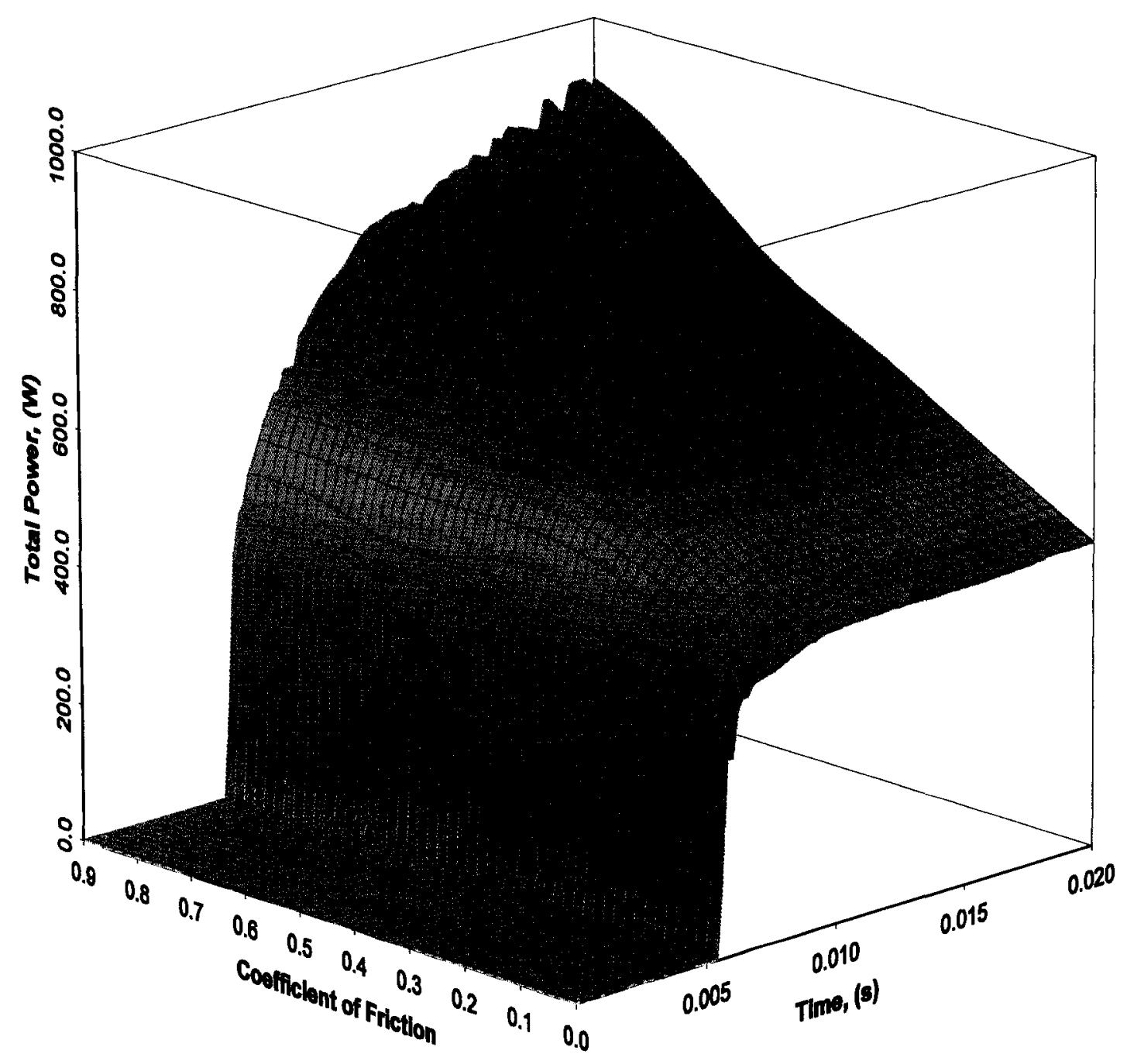

Figure 9.12: Surface plot of total power $\left(P_{t}\right)$ consumed, in units of $\mathrm{W}$, for metal cutting simulations in the Eulerian element formulation study, with workpiece modeled as Material Model 1. 


\subsection{RESULTS GENERATED FROM MATERIAL MODEL 2}

Material Model 2 was based on the Johnson-Cook constitutive equation, with constants obtained from work by Johnson and Cook [68], as discussed in Section 6.1.3.1. The interaction between the workpiece and tool was investigated with four different coefficients of friction varying between 0.0 and 0.4 , with processing time varying from 133 hours and 12 minutes to 136 hours and 28 minutes.

\subsubsection{Energy Balance}

The energy balance typical of simulations conducted with Material Model 2 is shown in Figure 9.13 and closely resembles the energy balance observed in the study of Material Model 1. Total energy was composed of both internal energy and sliding energy components, but the contribution of internal energy was much more significant. Although penetration, or sliding energy, could not be completely eliminated, it was present only in small amounts and was negligible. Once again, the internal energy was much greater than the kinetic energy and hence kinetic energy appeared to be approximately zero in Figure 9.13. The external work and total energy were equivalent up to $10.0 \mathrm{~ms}$, beyond which time the kinetic and internal energy in the outgoing workpiece material were omitted from the energy balance, and external energy exceeded total energy as previously described in Section 9.2.1.

\subsubsection{Deformation of the Workpiece}

Three simulations were completed with Material Model 2, each with a different coefficient of friction applied between the tool and workpiece. As with observations made on Material Model 1 in Section 9.2.2, increasing the coefficient of friction had a significant effect on chip geometry. The investigation studied friction coefficients of 0.0 , 0.2 , and 0.4 , and their effect on chip thickness. The results of the simulations are summarized in Figure 9.14 (a). The relationship between the coefficient of friction $(\mu)$ and the chip thickness $\left(t_{c}\right)$ is plotted in Figure 9.14 (b) and obeys the following quadratic equation:

$$
t_{c}=1.1475 \mu^{2}+1.227 \mu+1.0464
$$




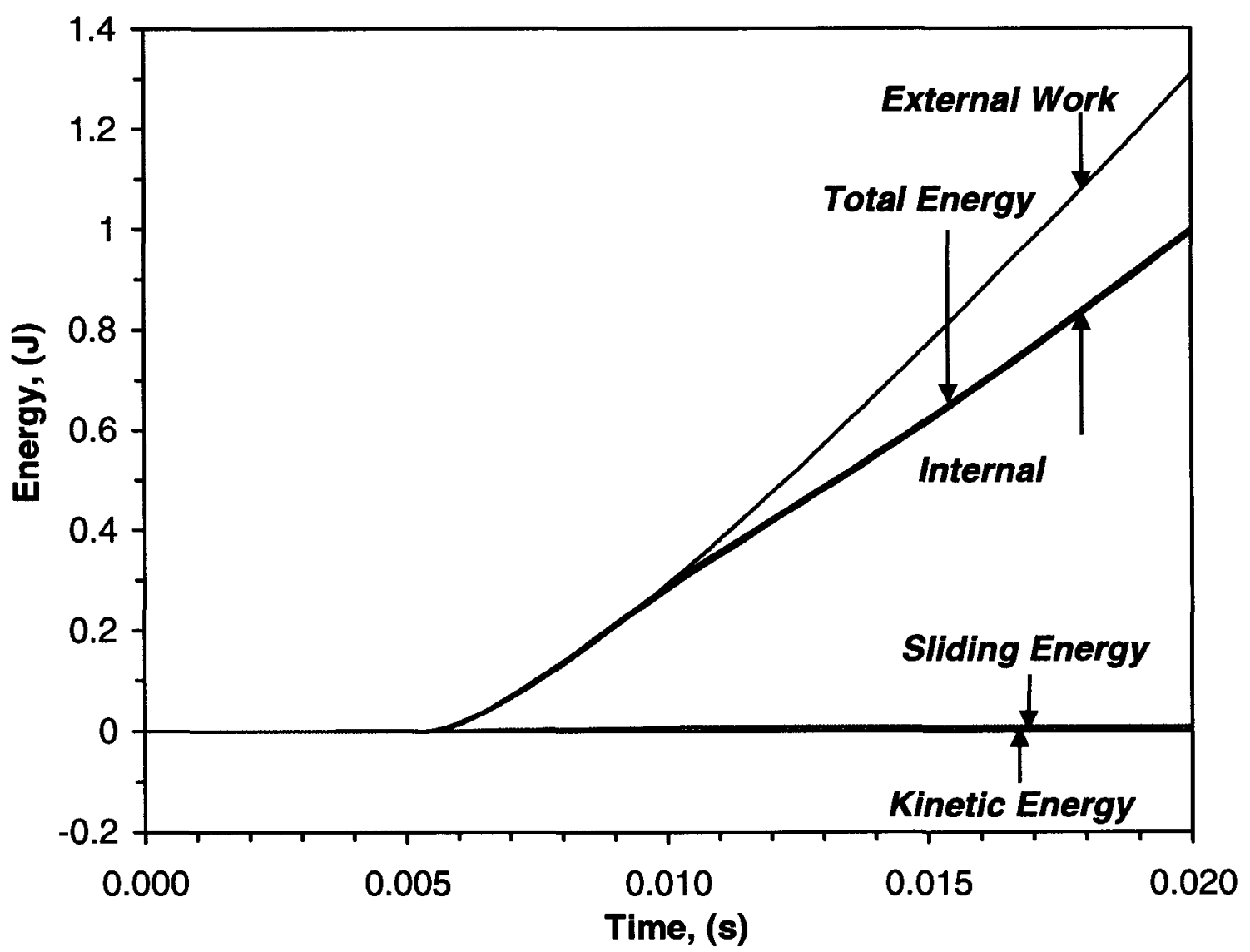

Figure 9.13: Energy balance typical of the metal cutting simulated in the Eulerian element formulation study, with workpiece modeled as Material Model 2. 
Figure 9.14 (b), when paired with Equation (9-2), showed that a coefficient of friction of 0.39 best replicated the experimental chip thickness of $1.7 \mathrm{~mm}$ observed in Figure 2.17. Subsequently, another simulation was completed employing a coefficient of friction equal to $\mu=0.39$. The geometry of the chip formed during this simulation was $1.7 \mathrm{~mm}$ and, as predicted, was in excellent agreement with the experimental chip thickness.

\subsubsection{Strain Distribution}

The strain distribution in material ahead of the tool tip, modeled with Material Model 2 and $\mu=0.39$, is presented in Figure 9.15. The strain in the workpiece varied between 0.0 , in regions where no plastic deformation was detected, and 8.3 , which was the maximum plastic strain accumulated in the material. An increase in strain was evident beneath the newly machined surface and at the rake face of the tool, due to the friction applied at the tool-workpiece contact. The maximum strain beneath the machined surface was 3.6, which occurred directly below the cutting line and decreased with increasing distance into the workpiece. Strain levels decreased to approximately 0.25 at $300 \mu \mathrm{m}$ beneath the machined surface; all traces of plastic deformation vanished at depths of $400 \mu \mathrm{m}$ beneath the machined surface. Maximum strain in the SDZ was 8.3 and occurred at $200 \mu \mathrm{m}$ above the tool tip. Significant plastic deformation within the SDZ was contained to $80 \mu \mathrm{m}$ of the rake face, which defined the width of the SDZ. The strain decreased with increasing distance from the rake face and ranged between 1.5 and 2.5 within the chip. The maximum strain within the PDZ was 7.2 and occurred at the tool tip, it decreased along the primary shear plane to a minimum of 0.5 at the chip root.

\subsubsection{Stress Distribution}

The computed values of the von Mises stress distribution corresponding to Material Model 2 and $\mu=0.39$ are presented in Figure 9.16. The maximum von Mises stress at the termination time was $438 \mathrm{MPa}$ and was located at the tool tip. Beneath the machined surface, a local maximum stress of $300 \mathrm{MPa}$ occurred adjacent to the cutting line. The stress decreased with increasing distance below the machined surface, and 


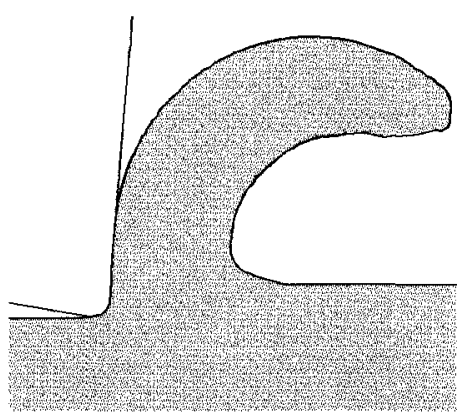

$\mu=0.0, t_{c}=1.05$

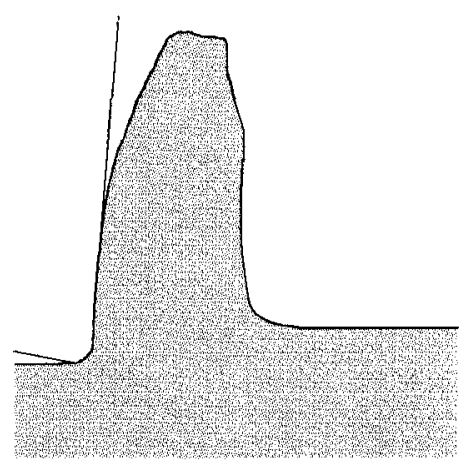

$\mu=0.2, t_{c}=1.34$

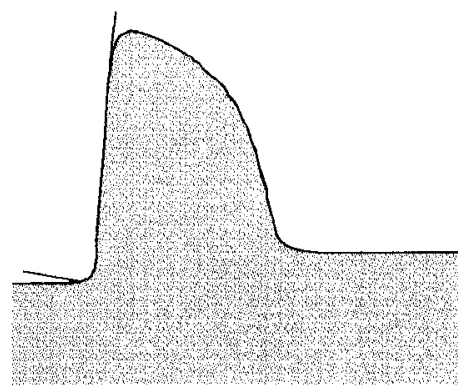

$\mu=0.4, t_{c}=1.72$

$500 \mu \mathrm{m}$

(a)

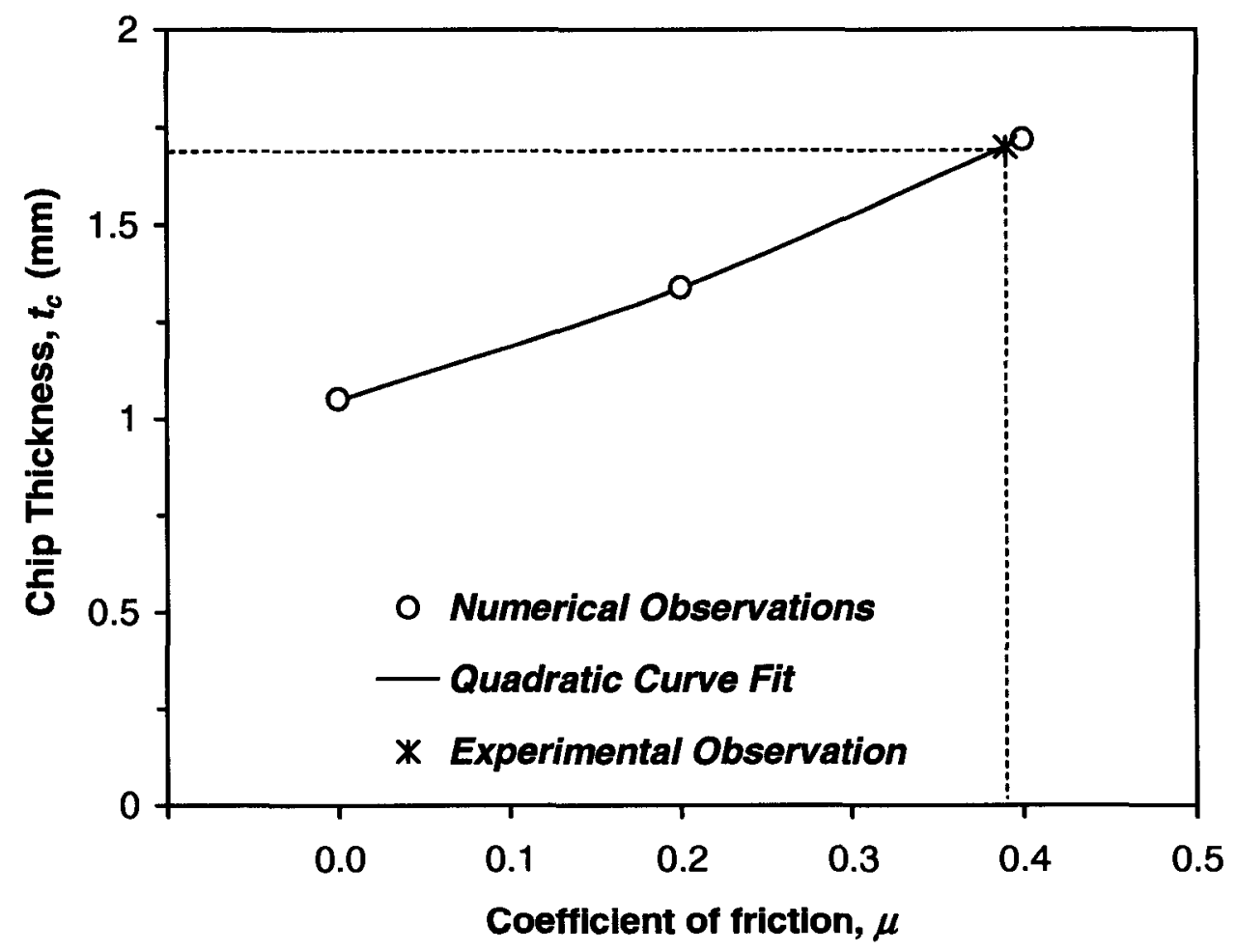

(b)

Figure 9.14: Investigation of friction coefficient effect on chip thickness for Material Model 2, shown are: (a) chip geometry with corresponding coefficients of friction, and (b) coefficient of friction vs. chip thickness plot, with experimental data noted. 


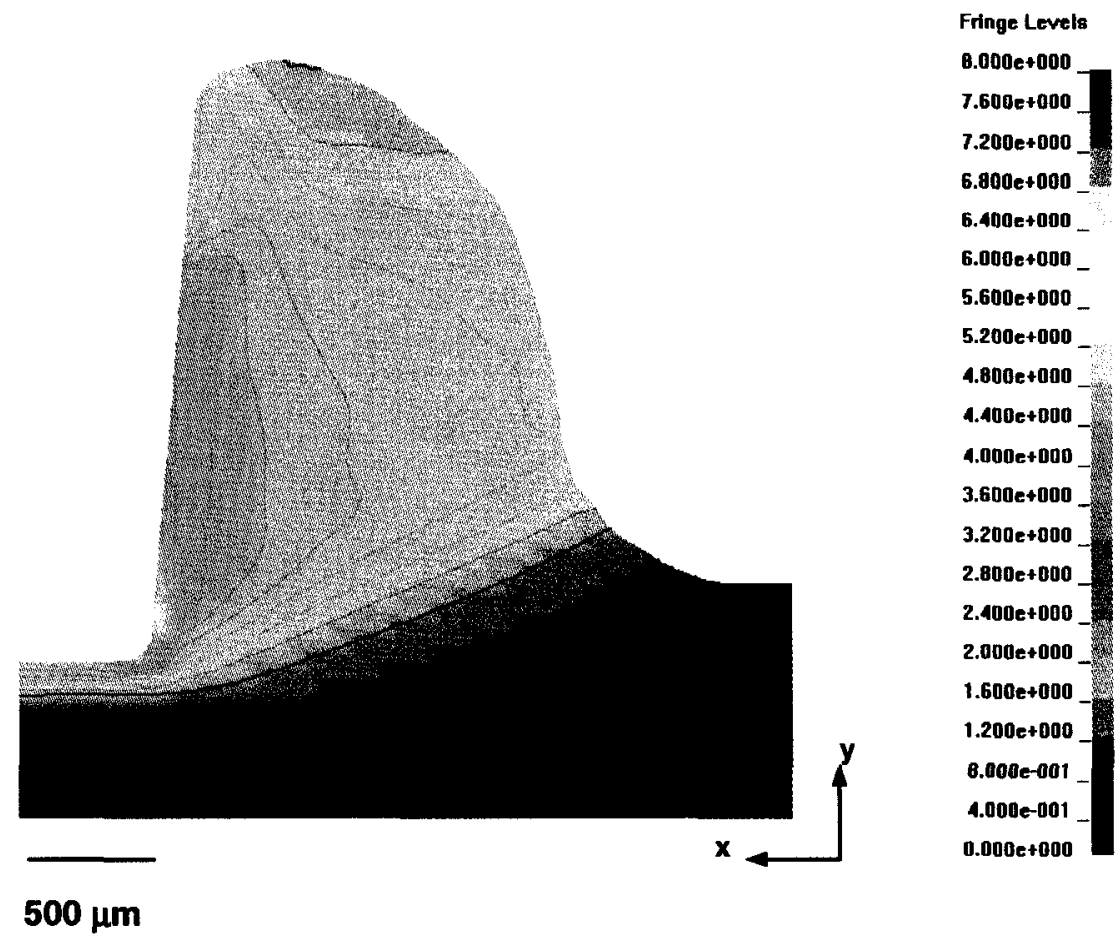

Figure 9.15: Strain distribution in the material ahead of the tool tip in the Eulerian element formulation study, with workpiece modeled as Material Model 2 and $\mu=0.39$.

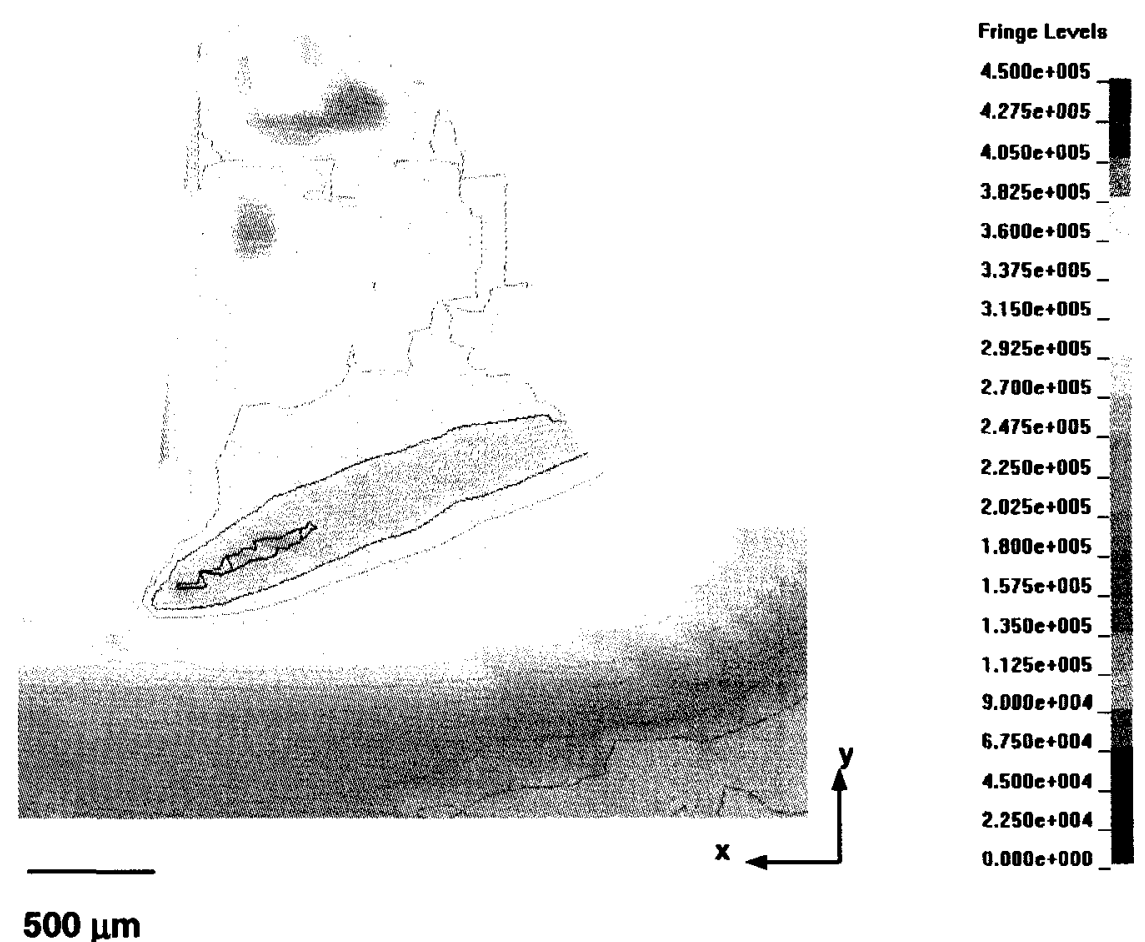

Figure 9.16: Stress distribution in the material ahead of the tool tip, in units of $\mathrm{kPa}$, in the Eulerian element formulation study, with workpiece modeled as Material Model 2 and $\mu=0.39$. 
stresses above yield strength extended to approximately $400 \mu \mathrm{m}$ below the cutting line, which was in agreement with the width of the plastic deformation zone predicted by the strain distribution. The concentration of increased stresses in the PDZ, ranging between 400 to $425 \mathrm{MPa}$, defined the zone width as approximately $600 \mu \mathrm{m}$. The stresses decreased above and below the PDZ with increasing distance. The stresses did not rise adjacent to the rake face, and thus the stress distribution did not indicate the existence of a SDZ, contrary to the strain distribution.

\subsubsection{External Work}

Work done in order to deform the workpiece was tracked with respect to time and the coefficient of friction applied at the chip-tool interface. The external work per unit depth of the model is presented in Figure 9.17. Up to a time of $5.5 \mathrm{~ms}$ no work was done because contact with the tool did not occur and the workpiece did not deform. Subsequent to the workpiece-tool contact, the external energy increased linearly to enable the deformation required for the continually forming chip, and was therefore maximum at termination. The external work also increased with an increasing coefficient of friction, similar to the trends observed in Material Model 1. The external work at termination time varied from $3.03 \mathrm{~J} \cdot \mathrm{mm}^{-1}$ for $\mu=0.0$ to $4.68 \mathrm{~J} \cdot \mathrm{mm}^{-1}$ for $\mu=0.4$. For the friction coefficient of $\mu=0.39$, which best represented the experimental chip geometry, the external work at termination was $4.60 \mathrm{~J} \cdot \mathrm{mm}^{-1}$. 


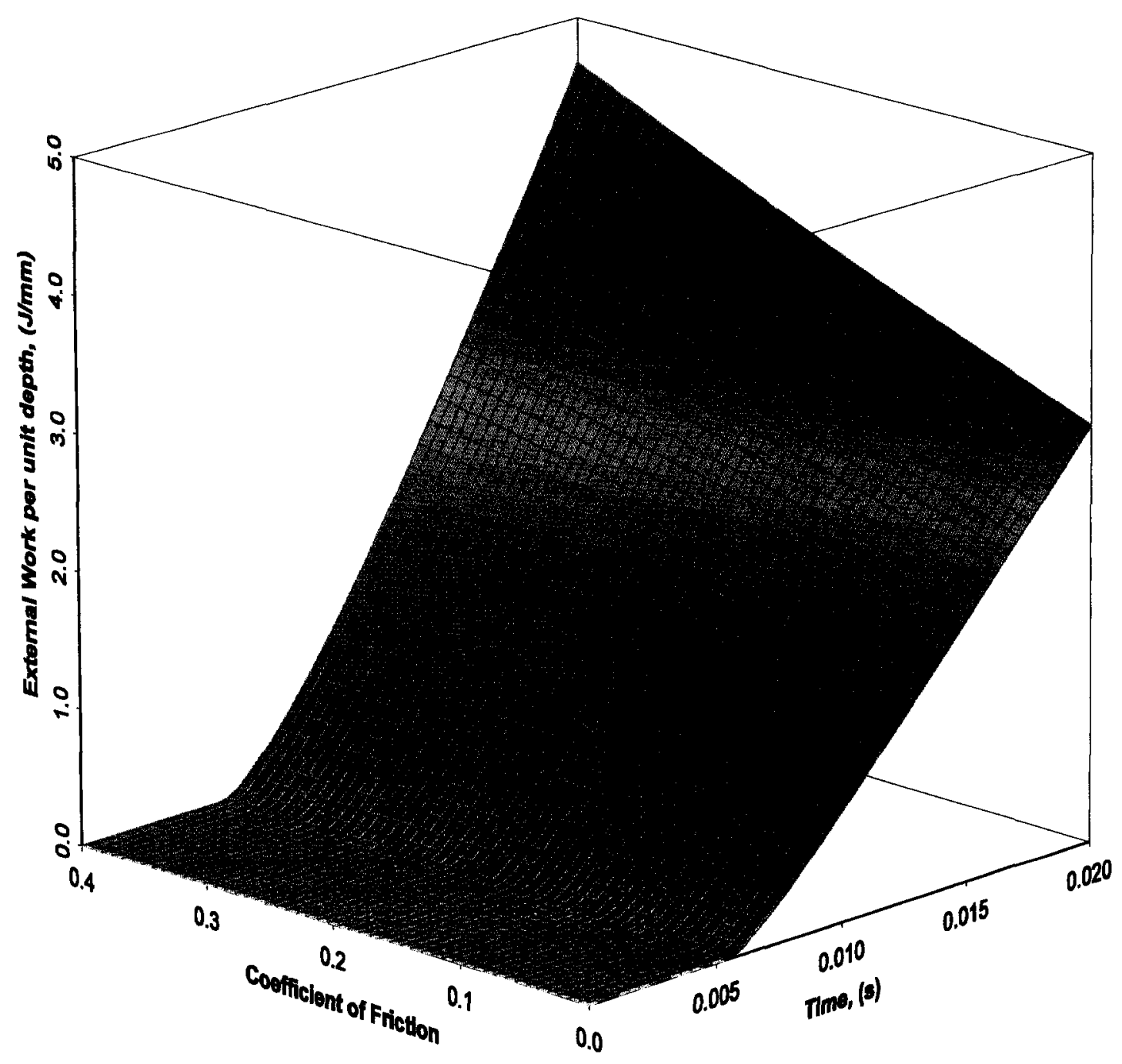

Figure 9.17: Surface plot of external work variation, in units of $\mathrm{J} \cdot \mathrm{mm}^{-1}$, with respect to time and friction, in the Eulerian element formulation study with workpiece modeled as Material Model 2. 


\subsubsection{Force and Power Predictions}

The forces produced at the tool-chip interface were analyzed with respect to time and the coefficient of friction applied at the contact interface. As discussed in Section 9.2.6, the x-component of the resultant contact force corresponded to the cutting force $\left(F_{c}\right)$, while the y-component and z-component corresponded to the thrust force $\left(F_{t}\right)$ and radial force $\left(F_{r}\right)$, respectively. Figure 9.18 presents the cutting forces, and Figure 9.19 presents the thrust forces both with respect to time and friction. The numerical force values were again multiplied by a factor of 11.1, to account for difference in contact areas between the numerical model and experimental tests, as was previously discussed in Section 9.2.6. The radial forces were negligible in comparison to cutting and thrust forces as was observed in the analysis of Material Model 1 and as stated by the assumptions of Piispanen [8]. The cutting and thrust forces remained zero until contact initiated between the tool and workpiece, which occurred at $5.5 \mathrm{~ms}$. Beyond $5.5 \mathrm{~ms}$, the forces rose rapidly until a steady state value was reached. Due to the longer time period required for the chip to reach a steady state formation, attaining a steady state force value was delayed in time with an increasing coefficient of friction. The cutting forces were significantly higher than the thrust forces throughout the cutting process, and both increased with higher coefficients of friction. The steady state value of the cutting forces increased from $1150 \mathrm{~N}$ for $\mu=0.0$ to $2000 \mathrm{~N}$ for $\mu=0.4$. The steady state thrust forces on the other hand increased from $517 \mathrm{~N}$ for $\mu=0.0$ to $963 \mathrm{~N}$ for $\mu=0.4$. For $\mu=0.39$, the coefficient of friction best replicating the experimental results, the cutting and thrust forces at the simulation termination were $1970 \mathrm{~N}$ and $941 \mathrm{~N}$, respectively.

The surface plot in Figure 9.20 was generated from the cutting force plot (Figure 9.18) utilizing Equation (2-10), and illustrates the total power consumption during the cutting process. Much like the cutting force, the total power increased at a fast rate until it reached a steady state value. As was found in the analysis of Material Model 1 and as shown by Figure 9.20, the power consumption increased as the friction between the tool and workpiece increased. The maximum power consumption was $690 \mathrm{~W}$ for $\mu=0.0$, and $1200 \mathrm{~W}$ for $\mu=0.4$. For $\mu=0.39$, the consumed power was $1182 \mathrm{~W}$. 


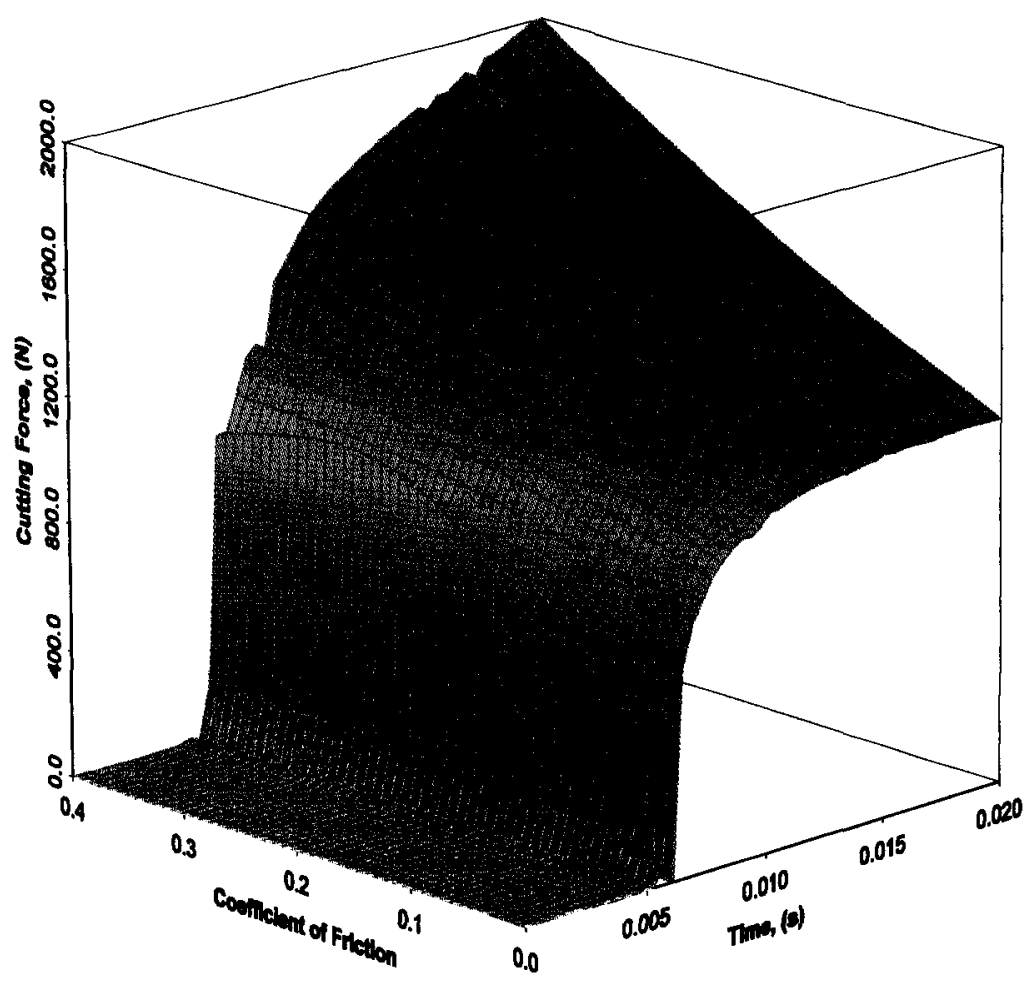

Figure 9.18: Surface plot of cutting force $\left(F_{c}\right)$ variation, in units of $\mathrm{N}$, with respect to time and friction, in the Eulerian element formulation study with workpiece modeled as Material Model 2.

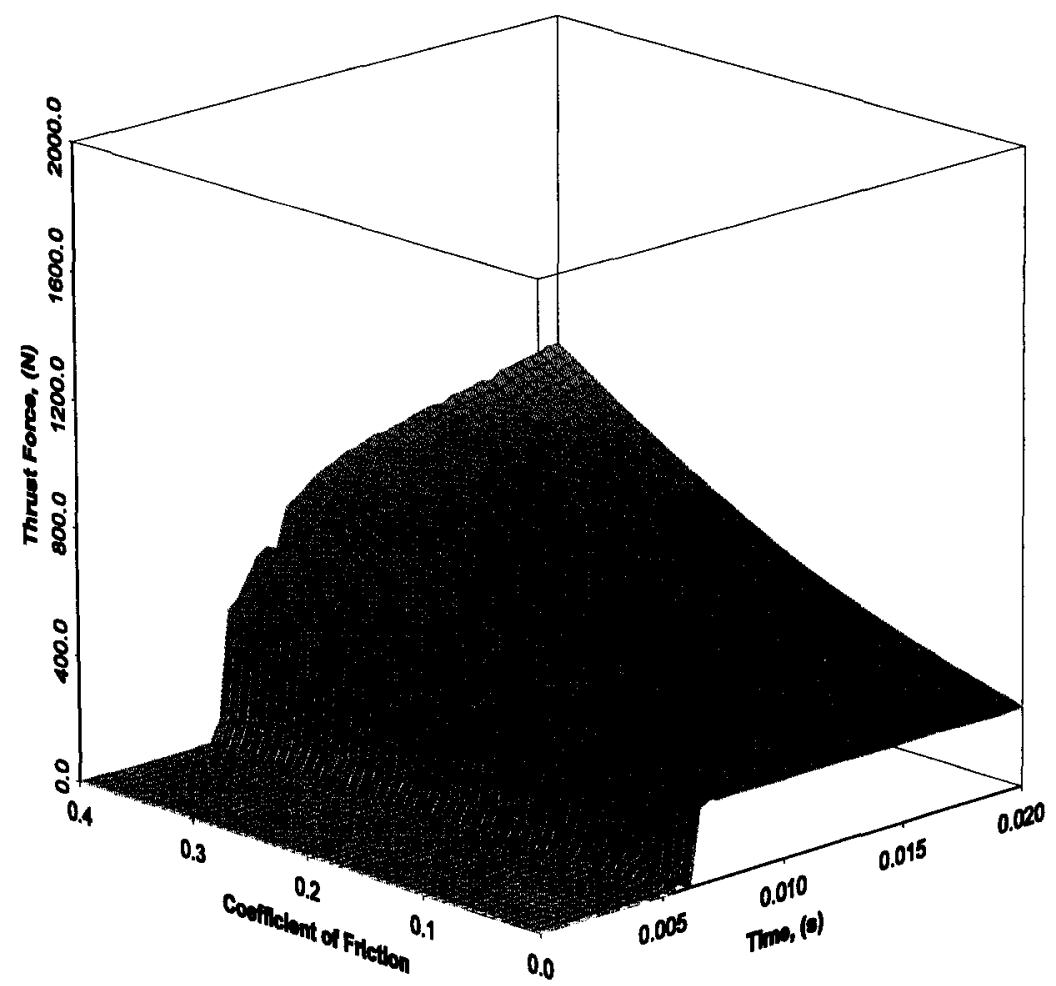

Figure 9.19: Surface plot of the thrust force $\left(F_{t}\right)$ variation in units of $\mathrm{N}$, with respect to time and friction, in the Eulerian element formulation study with workpiece modeled as Material Model 2. 


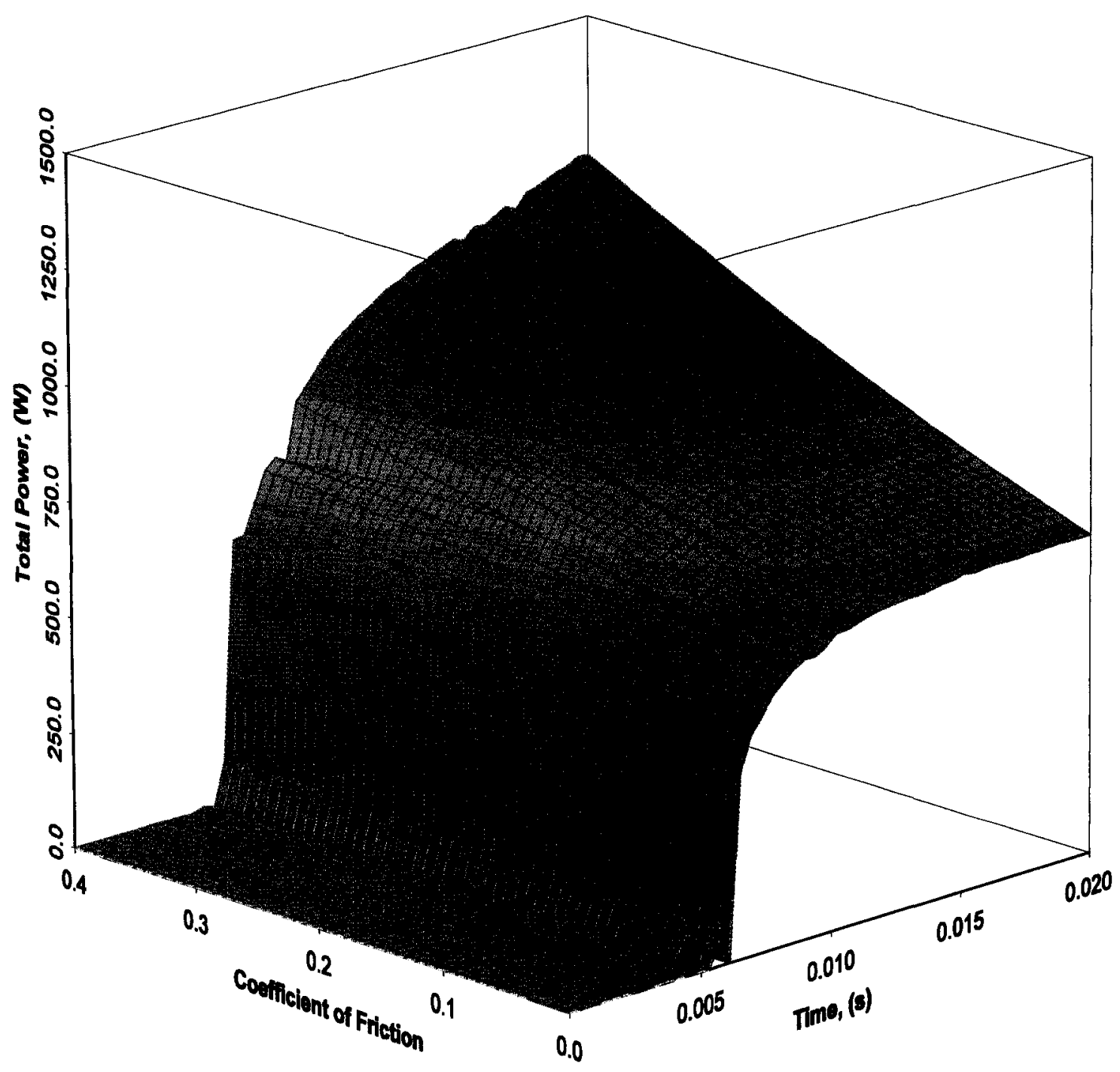

Figure 9.20: Surface plot of total power $\left(P_{t}\right)$ consumed, in units of $\mathrm{W}$, for metal cutting simulations in the Eulerian element formulation study with workpiece modeled as Material Model 2. 


\subsection{RESULTS GENERATED FROM MATERIAL MODEL 3}

The final material model, Material Model 3, described the stress-strain flow curve by a series of points fitted to the experimental data, as discussed in Section 6.1.3.2. The processing time for the simulation was 143 hours and 9 minutes.

\subsubsection{Energy Balance}

The energy balance associated with the simulation conducted for Material Model 3, is shown in Figure 9.21 and closely resembles the energy balance observed in the study of Material Models 1 and 2. All energies remained zero until the time when contact occurred between the tool and workpiece. Beyond initial contact, internal and total energy were equivalent to each other, while kinetic energy was negligible in comparison and appears to be zero in Figure 9.21. Penetration, represented by sliding energy, was also negligible in this investigation. After contact occurred, internal energy slightly exceeded external work. After $10.0 \mathrm{~ms}$, when portions of the workpiece material exited the airmesh, the total energy slope decreased and eventually external work exceeded the total energy, which is a concept described in detail in Section 9.2.1.

\subsubsection{Deformation of the Workpiece}

Material Model 3 was developed from the experimental flow curve (Figure 2.20), and is assumed to account inherently for frictional effects, as well as strain rate and temperature effects. Hence the coefficient of friction was specified as zero within the contact definition. The chip geometry is presented in Figure 9.22. The numerical chip thickness of approximately $1.6 \mathrm{~mm}$ was in good agreement with the experimental chip thickness of $1.7 \mathrm{~mm}$, thereby validating the assumption that frictional effects are in some way accounted for within the material model. 


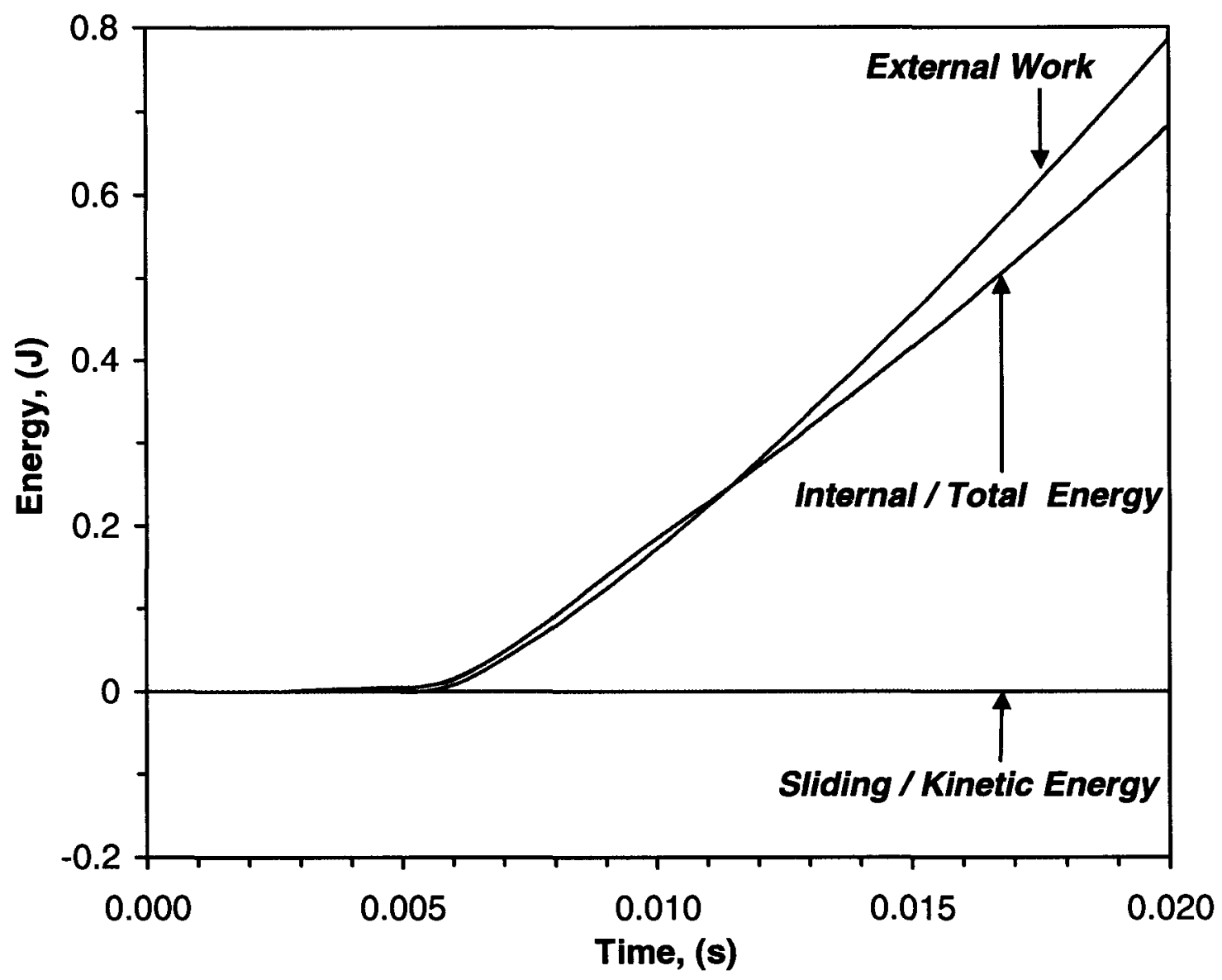

Figure 9.21: Energy balance typical of the metal cutting simulated in the Eulerian element formulation study, with workpiece modeled as Material Model 3.

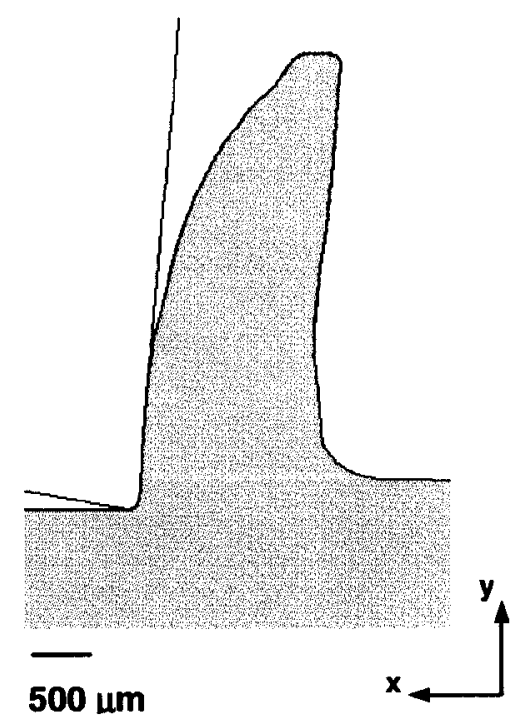

Figure 9.22: Geometry of the chip formed by the simulation in the Eulerian element formulation study with workpiece modeled as Material Model 3. 


\subsubsection{Strain Distribution}

The distribution of strain in the material ahead of the tool tip is presented in Figure 9.23. The strain within the workpiece varied between 0.0 in zones where plastic deformation did not occur, and a maximum strain of 5.6 at the tool tip. A strain increase was observed adjacent to the rake face and at the machined surface, due to the displacement of the material at the tool tip and subsequent chip formation. The maximum strain in the machined surface was 3.5 and occurred adjacent to the cutting line. The strain beneath the machined surface declined with increasing distance from the cutting line, decreasing to a strain of 0.05 at depths of approximately $300 \mu \mathrm{m}$ and diminishing completely below depths of $400 \mu \mathrm{m}$. The strain decreased along the primary shear plane, from a maximum of 5.6 at the tool tip to a minimum of 0.5 at the chip root. The SDZ was contained to a distance of approximately $80 \mu \mathrm{m}$ from the rake face. The strain within the SDZ varied between 4.5 adjacent to the rake face and 3.5 on the perimeter of this region. Outside of the SDZ and in the chip, the strain varied between 1.5 and 2.5 .

\subsubsection{Stress Distribution}

The distribution of von Mises stresses computed via Material Model 3 is shown in Figure 9.24. The maximum stress of $410 \mathrm{MPa}$ was located at the tool tip and corresponded to the location of maximum strain. The stresses increased adjacent to the machined surface and rake face of the tool, which was also consistent with observed strain increases. The maximum stress within the machined surface was $360 \mathrm{MPa}$ and was located along the cutting line. The stresses decreased with increasing distance from the cutting line, and below an approximate depth of $400 \mu \mathrm{m}$ they were equal to the yield strength of the material. The maximum stress within the SDZ was approximately $380 \mathrm{MPa}$. Contrary to the observations made in Material Models 1 and 2, high stresses were not concentrated within the PDZ; the stresses decreased along the primary shear plane from a maximum at the tool tip to a minimum of approximately $260 \mathrm{MPa}$ at the chip root. The width of the PDZ was approximated as $600 \mu \mathrm{m}$, dictated by the concentration of stress iso-contours between $230 \mathrm{MPa}$ and $330 \mathrm{MPa}$. 


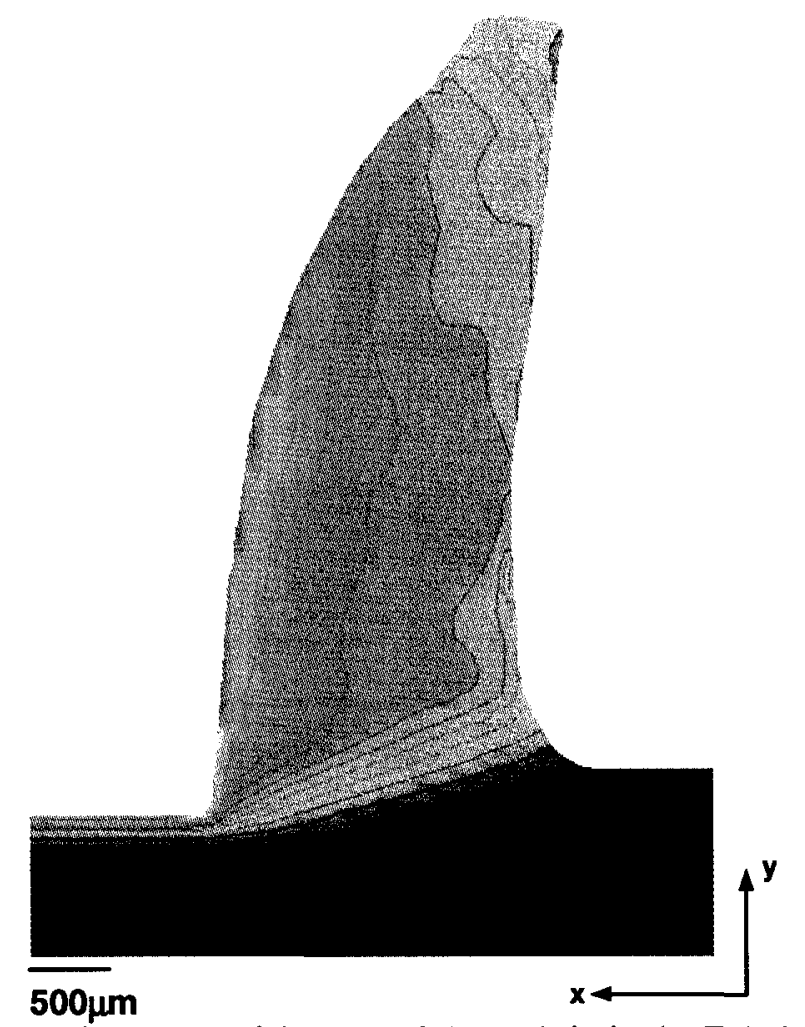

Fringe Levels
$6.000 e+000$
$5.700 e+000$
$5.400 e+000$
$5.100 e+000$
$4.800 e+000_{2}$
$4.500 e+000$
$4.200 e+000$
$3.900 e+000$
$3.600 e+000$
$3.300 e+000$
$3.000 e+000$
$2.700 e+000$
$2.400 e+000$
$2.100 e+000$
$1.000 e+000$
$1.500 e+000$
$1.200 e+000$

Figure 9.23: Strain distribution in the material ahead of the tool tip in the Eulerian element formulation study with workpiece modeled as Material Model 3.

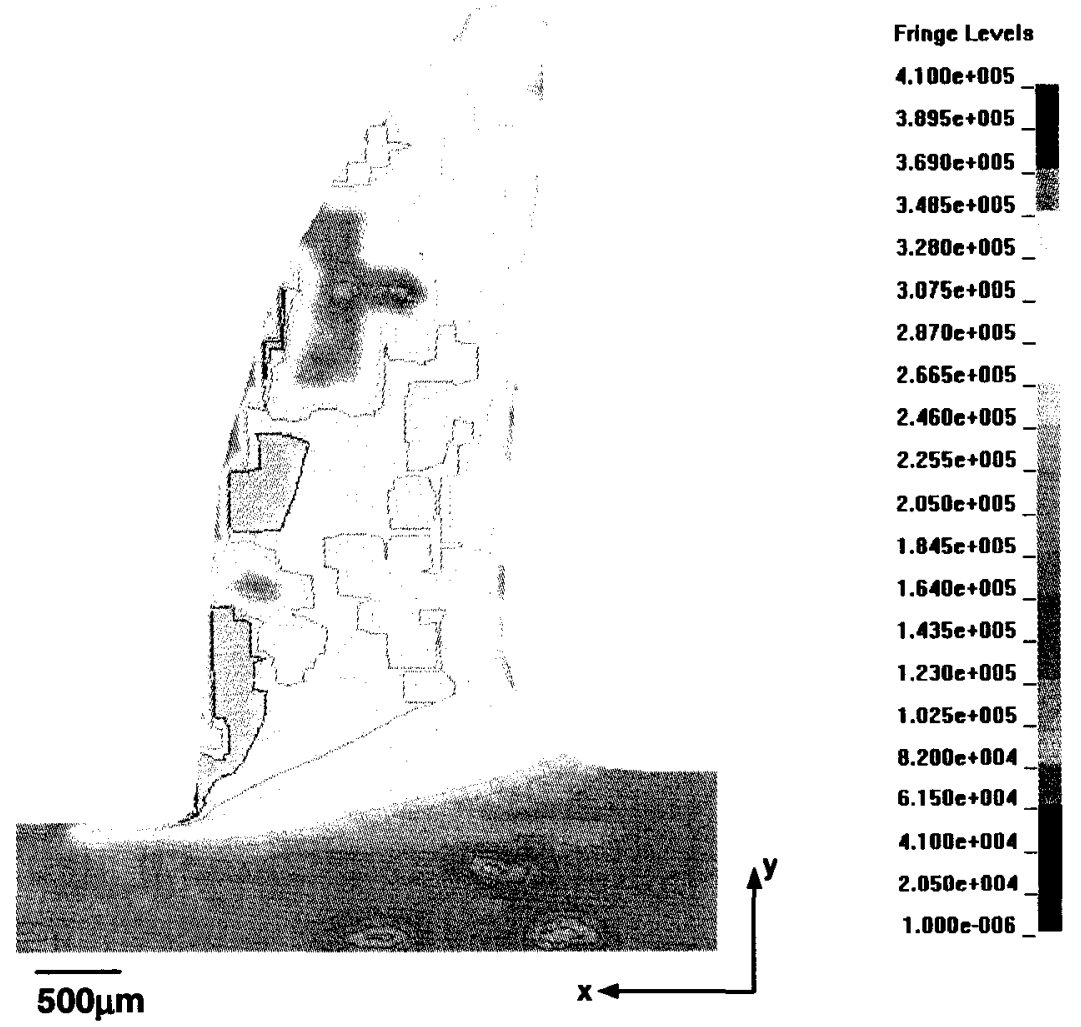

Figure 9.24: Stress distribution in the material ahead of the tool tip, in units of $\mathrm{kPa}$, in the Eulerian element formulation study with workpiece modeled as Material Model 3. 


\subsubsection{External Work}

External work per unit model depth is shown with respect to time in Figure 9.25. Up to a time of $5.5 \mathrm{~ms}$, when contact first occurred between the tool and workpiece, external energy remained zero because work is only required to deform the workpiece. Beyond the time of $5.5 \mathrm{~ms}$, external work increased linearly with progression of workpiece deformation. External work was maximum at termination time and was equal to $5.20 \mathrm{~J} \cdot \mathrm{mm}^{-1}$.

\subsubsection{Force and Power Predictions}

The forces produced at the tool-chip interface were analyzed with respect to time and are illustrated in Figure 9.26. The difference in experimental wall thickness $(3.0 \mathrm{~mm})$ and model depth $(0.27 \mathrm{~mm})$ was accounted for by multiplying the force values by a factor of 11.1. All forces remained zero until a time of first contact with the tool. The radial force or the z-component of the contact resultant force was negligible beyond $5.5 \mathrm{~ms}$ and in agreement with the assumptions of Piispanen [8]. The cutting and thrust force rose rapidly beyond the time of $5.5 \mathrm{~ms}$ and reached a more constant value once the metal cutting process reached a steady state stage. The cutting force was increasingly larger than the thrust force with time. At the simulation termination, the thrust force was $209 \mathrm{~N}$ while the cutting force was over six times larger at $1332 \mathrm{~N}$.

The cutting force was used to calculate the power consumption of the cutting process via Equation (2-10), which is illustrated in Figure 9.27. The trends observed in power consumption were identical to those described for the cutting force due to the direct nature of the relationship between the two variables. The maximum power consumption of $799 \mathrm{~W}$ was computed at termination time when the process was roughly estimated to be steady state. 


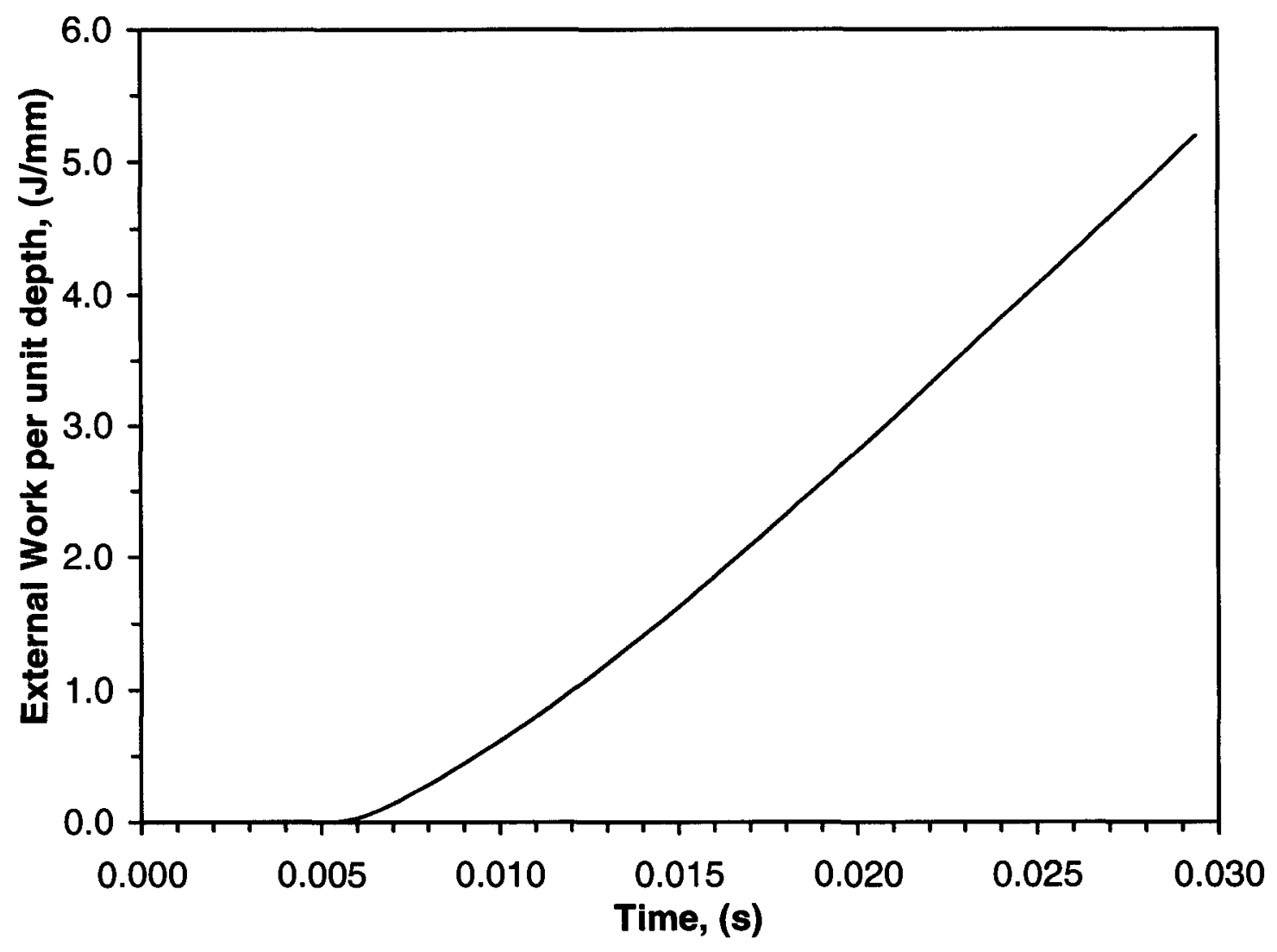

Figure 9.25: External work variation, in units of $\mathrm{J} \cdot \mathrm{mm}^{-1}$, in the Eulerian element formulation study with workpiece modeled as Material Model 3. 


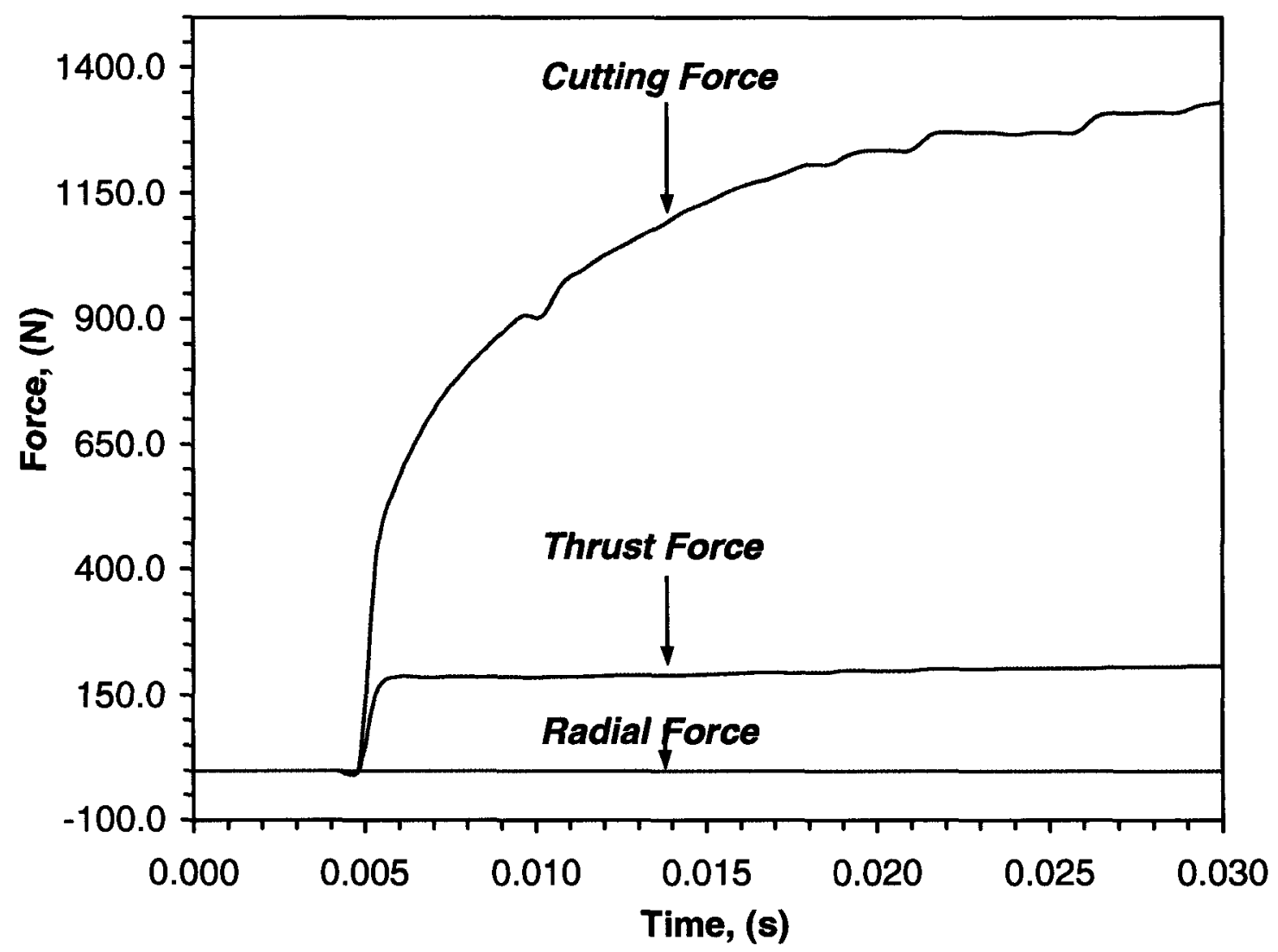

Figure 9.26: Cutting $\left(F_{c}\right)$, thrust $\left(F_{t}\right)$ and radial $\left(F_{r}\right)$ forces, in units of $\mathrm{N}$, in the Eulerian element formulation study with workpiece modeled as Material Model 3. 


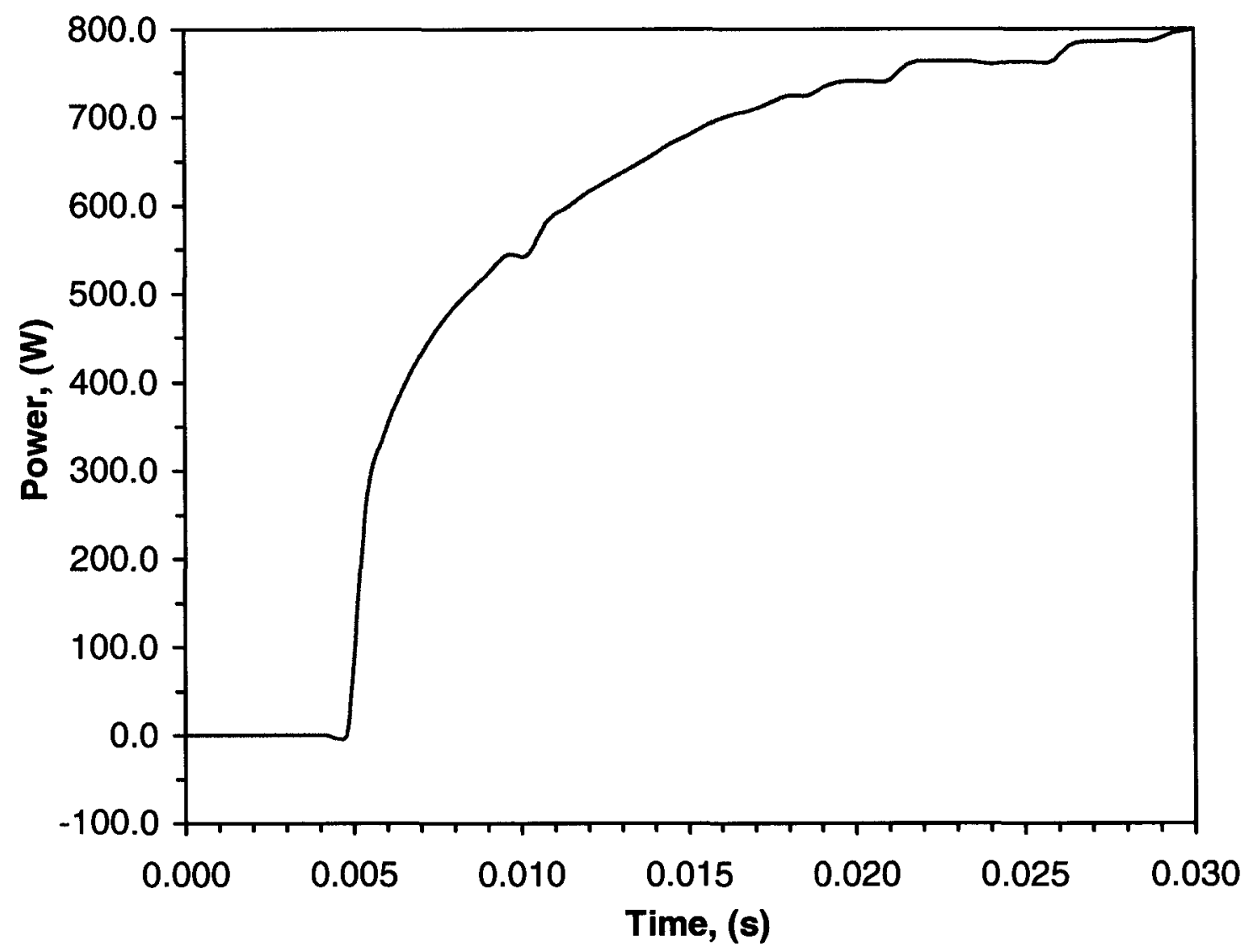

Figure 9.27: Total power $\left(P_{t}\right)$ consumed, in units of $\mathrm{W}$, for the metal cutting simulation in the Eulerian element formulation study with workpiece modeled as Material Model 3. 


\section{CHAPTER 10}

\section{Discussion of Results}

\subsection{LAGRANGIAN ELEMENT FORMULATION}

The results of the Lagrangian element formulation study for the steady state chip formation were presented in Chapter 7. The study included a mesh sensitivity analysis and an investigation into the effectiveness of adapting the workpiece mesh to control distortion and improve reliability of results.

It was evident that the Lagrangian element formulation was incapable of supporting the high deformation required to displace the material ahead of tool tip. Although it is commonly understood that a finer mesh can help to produce more accurate results, here the smaller elements at the tool tip resulted in greater element distortion during interaction with the tool. The stress and strain distributions, shown in Figures 7.3 and 7.4, respectively, showed a concentration of material displacement in the PDZ, which was consistent with the shearing of material along the primary shear plane occurring in experiments. Correlation of the values of stresses, strains and forces, to experimental observations was not valid since the force applied to the tool in the simulations was scaled down (Section 4.1.4) to control the workpiece mesh distortion at the tool tip.

The solution to the problem of mesh distortion used by other researchers studying metal cutting with the Lagrangian element formulation has been either to apply a separation criteria to enable the chip and machined surface to separate, or to continually adapt the mesh. Use of a separation criterion, although effective, is an unrealistic portrayal of the mechanisms of chip formation; it simulates a fracture of the material instead of a material deformation. This method was not studied in this project due to its inappropriateness. An attempt was made to adapt the workpiece fully and partially (around the tool tip) in an effort to control the mesh distortion, but this method generated other difficulties. Since a whole new mesh is generated during adaptivity, all nodal constraints placed on the workpiece were lost within the first adaptive step, which resulted in unusual workpiece deformation. Several different constraint methods were analyzed but all faltered under the application of adaptivity. 
The lack of success in the use of adaptivity, and the questionable validity of using a separation criterion, resulted in the abandonment of the Lagrangian element formulation and to the consideration of a new approach. This approach consisted of using the ALE element formulation and ALE smoothing in efforts to reduce the mesh distortion at the tool tip.

\subsection{ALE ELEMENT FORMULATION}

The FE model of steady state chip formation showed considerable improvement in mesh distortion occurring at the tool tip when Figure 8.2 (from the ALE results) and Figure 7.2 (from the Lagrangian results) were compared. However, the ALE smoothing was insufficient to allow chip formation. At a certain deformation, the workpiece bowed under the load being applied by the tool, and bending occurred as evident in Figure 8.2. Despite several attempts to optimize the smoothing weight factors (Section 2.2.2), the bending in the workpiece persisted. The FE model of incipient chip formation was also unsuccessful in the modeling of metal cutting. Although several adjustments to the ALE smoothing were made, the mesh distortion at the tool tip was so great that erroneous termination resulted for all investigations.

Since mesh distortion and erroneous termination could not be eradicated, the FE model was not successful in predicting chip formation; as such experimental and numerical correlation was not completed. Instead of continuing to attempt to make the ALE study operative, attention was turned towards the last possible element formulation, the Eulerian formulation. The Eulerian element formulation showed promise of eliminating mesh distortion and enabling the modeling of material flow around the tool without applying smoothing and artificial separation criterion. 


\section{I0.3 EULERIAN ELEMENT FORMULATION}

The Eulerian element formulation effectively modeled the metal cutting process without imposing any artificial failure criteria and without applying mesh adaptivity or smoothing. Capability of the material to move independently of the mesh enabled the Eulerian workpiece undergoing metal cutting to displace at the tool tip to form a chip and a machined surface. The Eulerian FE model was the only one studied in the present work that could efficiently model the cutting parameters and mechanism involved in chip formation. Extensive correlation was performed between all three material models investigated and the experimental findings reported by Elmadagli and Alpas [56]. Comparisons between numerical and experimental chip geometry, strain distributions, stress distributions, forces, and power consumption are summarized below.

\subsubsection{Deformation of the Workpiece}

The geometry of the chips formed by each of the material models studied during the metal cutting simulations is shown in Figure 10.1 and was compared to the chip geometry formed during the experimental machining tests. The coefficient of friction used for Material Model $1(\mu=0.84)$ and Material Model $2(\mu=0.39)$ was that which best replicated the experimental geometry, and thus these numerically determined chip thicknesses were in agreement with the experimental results. The disadvantage of these material models is that a range of coefficients of friction had to be considered before determining which value of $\mu$ was the most appropriate for the analysis. It is very difficult to presume the validity of the coefficients of friction for Materials 1 and 2, since they estimate a value for the combination of the sticking and sliding region. The friction between copper and silicon nitride can range between 0.2 and 0.6 for humidity levels of $95 \%$ to $10 \%$ [67] respectively, which is applicable to the analysis of the sliding region. In the sticking region, where the workpiece can seize to the tool, the friction reaches values much greater than 1.0. In combination, sticking and sliding regions can produce a mean friction coefficient of either 0.84 or 0.39 , as predicted by Material Models 1 and 2, respectively. Therefore, it is unfeasible to declare whether Material Model 1 or 2 is more 


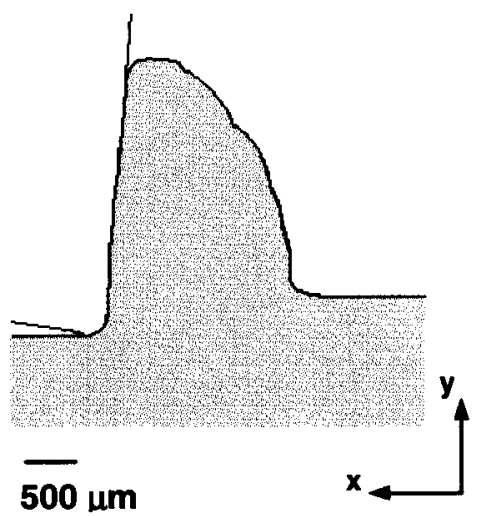

(a)

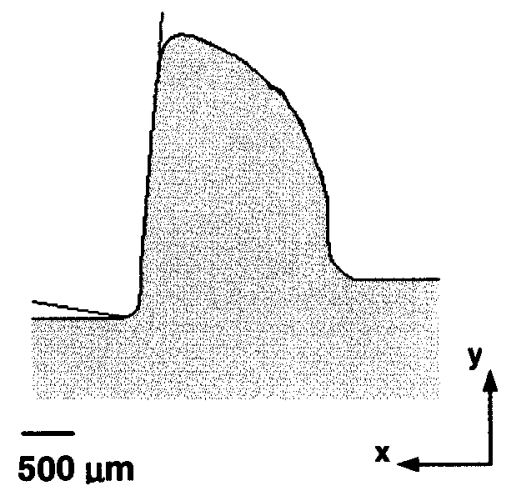

(b)

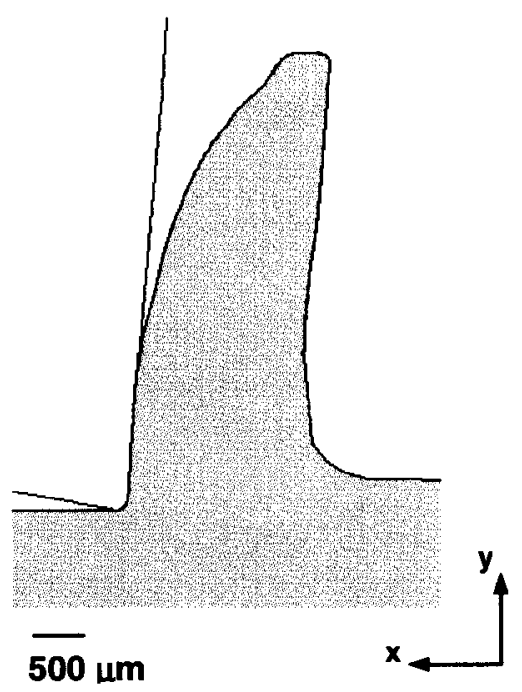

(c)

Figure 10.1: Chip geometry formed during simulation of: (a) Material Model 1 with $\mu=0.84$ at termination time of $\mathrm{t}=20.0 \mathrm{~ms}$, (b) Material Model 2 with $\mu=0.39$ at termination time of $\mathrm{t}=20.0 \mathrm{~ms}$, and (c) Material Model 3 at termination time of $\mathrm{t}=30.0 \mathrm{~ms}$. 
accurate on the basis of which coefficient of friction reproduces the experimental chip geometry.

For Material Model 3, a chip thickness of $1.6 \mathrm{~mm}$ was produced without considering friction and was within $6 \%$ of the experimental chip thickness of $1.7 \mathrm{~mm}$. Since it is clear that friction must exist between the tool and the workpiece, and Material Model 3 generated accurate results without considering friction in the contact algorithm, it can be deduced that friction must be in some way inherently included within the stress-strain behaviour of the material as originally assumed (Section 6.1.5). The capabilities of Material Model 3 to predict accurately the chip formation process, without the user having to estimate a friction coefficient, poses a definite advantage of material Model 3 over both Material Models 1 and 2.

\subsubsection{Strain Distribution}

The distribution of strain in the workpiece for each of the studied material models is shown in Figures 10.2 through 10.5. Figure 10.2 shows the strains in Material Model 1 with $\mu=0.84$; Figure 10.3 shows those in Material Model 2 with $\mu=0.39$; and Figure 10.4 shows the strains in Material Model 3. The numerical strain distributions are accompanied by the experimental results, shown in Figure 10.5, which were obtained from reference [56].

\section{Machined Surface}

In the machined surface, the numerical models predicted a decrease in strain with increasing distance below the cutting line. The maximum strain in the machined surface for Material Model 3 was 3.5, slightly lower then that predicted by the other two models, which was 3.6. At depths of $300 \mu \mathrm{m}$ below the cutting line, the strain in Material Model 2 was 0.25 , which is significantly higher then the strain of 0.025 in Material Model 1 and strain of 0.05 in Material Model 3. Plastic deformation in the machined surface was contained within a depth $350 \mu \mathrm{m}$ in Material Model 1, and to $400 \mu \mathrm{m}$ in Material Models 2 and 3.

The strain in the machined surface of the experimental sample reached values of 8.1 very close to the cutting line. The contour equivalent to a strain of 3.64 occurred at a 


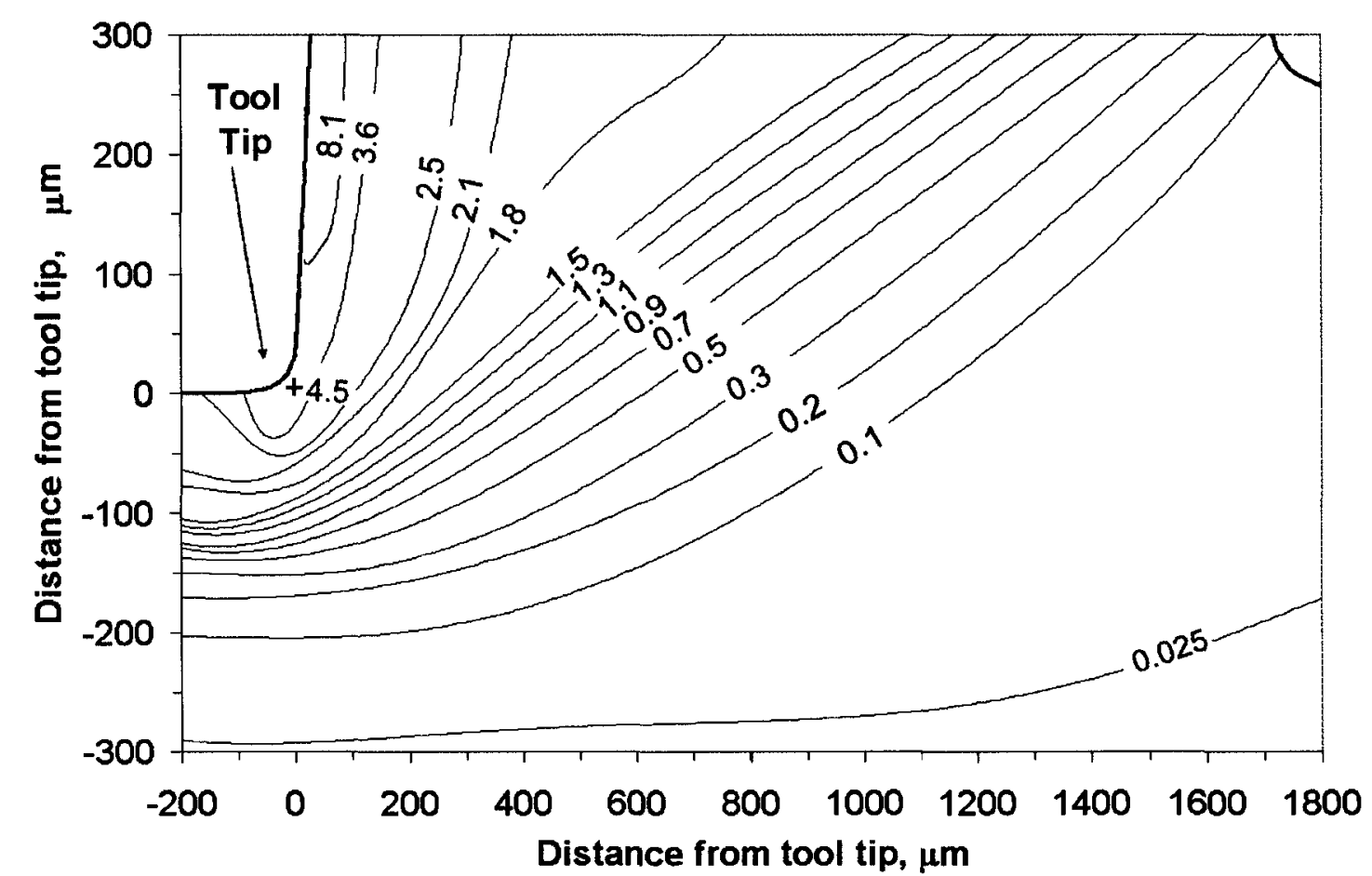

Figure 10.2: Contours of equivalent strain according to Material Model 1 with $\mu=0.84$ at $\mathrm{t}=20.0 \mathrm{~ms}$.

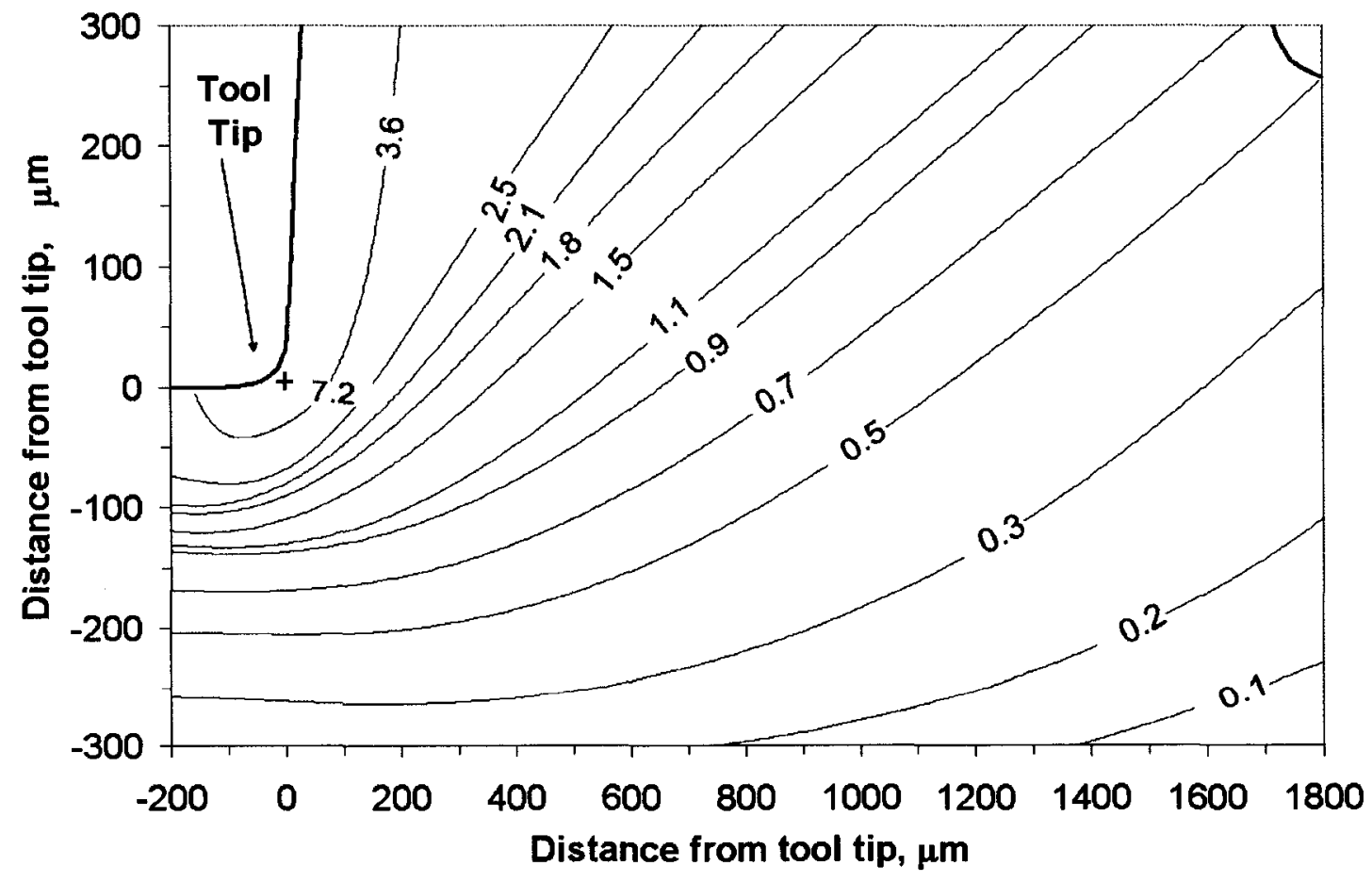

Figure 10.3: Contours of equivalent strain according to Material Model 2 with $\mu=0.39$ at $\mathrm{t}=20.0 \mathrm{~ms}$. 
depth of approximately $50 \mu \mathrm{m}$, which corresponded well to the depth of the 3.5 contour for Material Model 3 and 3.6 for Material Models 1 and 2. At $300 \mu \mathrm{m}$, the experimental strain was approximately 0.05 and plastic deformation extended to $400 \mu \mathrm{m}$ beneath the cutting line; these results were well predicted by all the material models studied but were best replicated by Material Model 3.

\section{Secondary Deformation Zone (SDZ)}

For all studied material models the strain in the SDZ was highest adjacent to the tool rake face and decreased towards the free surface of the chip. The maximum strain in Material Model 1 occurred within the SDZ approximately $400 \mu \mathrm{m}$ above the tool tip (as noted in Section 9.2.3), which corresponded to $(50 \mu \mathrm{m}, 400 \mu \mathrm{m})$ where the $\mathrm{x}$ and $\mathrm{y}$ coordinates were measured with reference to the tool tip. The value of the maximum strain in Material Model 1 was 7.5. The maximum strain in Material Model 2 was predicted as 8.3 and occurred at $(25 \mu \mathrm{m}, 200 \mu \mathrm{m})$, also in the SDZ. Contrary to Material Models 1 and 2, Material Model 3 predicted the location of maximum strain directly at the tool tip $(0 \mu \mathrm{m}, 0 \mu \mathrm{m})$ with a value of 5.6. The SDZ was identified as the region adjacent to tool rake face, where there was an increase in strains. The width of the SDZ was assumed as the distance between the rake face and the contour of strain 3.5 or 3.6 , defining a region of high strain concentration. By this definition, the SDZ widths were approximated as $100 \mu \mathrm{m}$ for Material Model 2, and $80 \mu \mathrm{m}$ for Material Models 1 and 3. Beyond the SDZ, the strain within the chip ranged between 1.5 and 2.5 for all material models.

The experimental results show that the maximum strain in the workpiece, occurred above the tool tip adjacent to the rake face, which was well represented by Material Models 1 and 2. The value of experimental maximum strain was 8.1, which is within $7 \%$ of the predictions in Material Model 1, and within 2\% of those in Material Model 2. The location of maximum strain in the experimental results was inconsistent with that observed in Material Model 3, and was under-predicted by $30 \%$ in its value. The definition of the SDZ width was consistent with that used in the numerical results, or the distance between the rake face and the strain contour of 3.64. The SDZ in Material Models 2 and 3 predicted the experimental width of $60 \mu \mathrm{m}$ to a higher precision then 
Material Model 1, which varied by nearly $70 \%$. The experimental strain attained within the chip was 1.5 , which was in good correlation with the strain in the numerical analyses.

\section{3. $\quad$ Primary Deformation Zone (PDZ)}

The variations of strain within the PDZ were consistent for all studied material models, i.e., the strain was maximum at the tool tip and decreased along the primary shear plane to reach a minimum strain at the chip root. The values of strain at the tool tip for Material Models 1 and 2 were 4.5 and 7.2 respectively, but were only the local maximum strain values. The tool tip strain in Material Model 3 was 5.6, which was the absolute maximum strain attained in the workpiece. For Material Model 1, the strain of 4.5 at the tool tip $(0 \mu \mathrm{m}, 0 \mu \mathrm{m})$ decreased along the primary shear plane, to a value of 0.1 at the chip root $(1700 \mu \mathrm{m}, 250 \mu \mathrm{m})$. In Material Model 2, a strain of 7.2 at $(0 \mu \mathrm{m}, 0 \mu \mathrm{m})$ decreased to 0.5 at $(1700 \mu \mathrm{m}, 250 \mu \mathrm{m})$, along the primary shear plane. Material Model 3 accumulated a maximum strain of 5.6 at $(0 \mu \mathrm{m}, 0 \mu \mathrm{m})$, which decreased along the primary shear plane to 0.5 at $(1700 \mu \mathrm{m}, 250 \mu \mathrm{m})$. The calculated strain gradient along the primary shear plane was noted to be the greatest in Material Model 2, and the lowest in Material Model 1.

The tool tip strain in the experimental results was reported as 3.65 [56] and decreased along the primary shear plane to a minimum of 1.5 at the chip root $(1700 \mu \mathrm{m}, 250 \mu \mathrm{m})$, which was consistent with the trend observed in the PDZ of the numerical strain distributions. The strain gradient within the PDZ of the experimental strain distribution was much smaller then that observed in the numerical results.

In general, correlation between strain values for the numerical and experimental test methods showed that the trends in the machined surface, SDZ, and PDZ, were in good agreement. Material Model 3 showed the least accuracy on predicting the location of maximum strain, but was otherwise well capable of replicating experimental strains. Material Model 1 did not accurately approximate the SDZ width, and did not predict the location of the maximum strain. Of the three material models studied, Material Model 2 exhibited the best agreement with the experimental strain distribution. 


\subsubsection{Stress Distribution}

The numerical predictions of von Mises stress distributions are shown in Figures 10.6, 10.7, and 10.8, for Material Models 1, 2, and 3 respectively. For the purpose of numerical-experimental correlation, Figure 10.9 shows the experimental stress distribution that was generated from information provided in reference [56].

\section{Machined Surface}

For the machined surface, the numerical models predicted a decrease in stress with increasing depth below the cutting line, which was consistent with trends observed in strain distributions. These stresses in the machined surface were much higher in Material Model 3 at $360 \mathrm{MPa}$, than in Material Models 1 and 2 where the local maximum stress peaked at $260 \mathrm{MPa}$ and $300 \mathrm{MPa}$, respectively. Stresses above the yield level were contained within the depth of the plastic deformation zones as predicted by locations of plastic strains; the depth of plastic deformation was $350 \mu \mathrm{m}$ for Material Model 1, and $400 \mu \mathrm{m}$ for Material Models 2 and 3.

Trends in the experimental stress distribution of the machined surface were consistent with those in the numerical analysis. The maximum stress detected within the machined surface of the experimental sample was $360 \mathrm{MPa}$, which was in excellent agreement with Material Model 3. Material Models 1 and 2 underestimated the maximum experimental stress in the machined surface by $28 \%$ and $17 \%$, respectively.

\section{Secondary Deformation Zone (SDZ)}

The distribution of stress in the SDZ of Material Models 1 and 2 was significantly different than that of Material Model 3. Material Model 3 showed that the stress in the SDZ was maximum adjacent to the tool rake face and decreased towards the free surface of the chip, which was similar to trends observed in the strain distributions. The maximum stress contour within the SDZ was $360 \mathrm{MPa}$ at a distance of approximately $100 \mu \mathrm{m}$ from the rake face. In Material Models 1 and 2, the stress distributions were inconsistent with what was observed in the corresponding strain distributions. The stress at the rake face of the SDZ was as low as $260 \mathrm{MPa}$ and $340 \mathrm{MPa}$ for Material Models 1 and 2, respectively, and increased with increasing distance from the rake face; this 
implies that the SDZ observed in the strain distribution does not exist according to the stress distribution. The increase in strain at the rake face should be accompanied by the increase in stress in this region, as observed in Material Model 3.

The experimental stress distribution showed an increase in stress at the rake face, which decreased towards the free surface of the chip. The highest stress contour was $360 \mathrm{MPa}$ at a distance of $120 \mu \mathrm{m}$ from the tool rake face. The experimental stress distribution was in best agreement with numerical results of Material Model 3; both showed a concentration of increased stresses at the rake face. Material Models 1 and 2 showed the exact opposite trends and did not agree with the experimental results.

\section{Primary Deformation Zone (PDZ)}

The location of the maximum von Mises stress for all three of the studied material models was at the tool tip. These maximum von Mises stress values for Material Models 1, 2, and 3 were $342 \mathrm{MPa}, 438 \mathrm{MPa}$, and $410 \mathrm{MPa}$ respectively. In Material Models 1 and 2 the stresses decreased when approaching the tool tip, which is inconsistent with the point location of maximum stress. Location of maximum stress in Material Model 3 was consistent with the remainder of the stress distribution, which showed an increase in stress as the tool tip was approached. The trends of stress distribution in the PDZ were similar for Material Models 1 and 2, but differed significantly for Material Model 3.

While increased stress in Material Model 3 was concentrated in the SDZ at the tool rake face, the stress concentration in the other two materials was within the PDZ. Along the primary shear plane of Material Model 1 , the stress at the tool tip $(0 \mu \mathrm{m}, 0 \mu \mathrm{m})$ was $260 \mathrm{MPa}$; it then increased to $275 \mathrm{MPa}$ at $(100 \mu \mathrm{m}, 50 \mu \mathrm{m})$; increased continually to a maximum of $330 \mathrm{MPa}$ at $(600 \mu \mathrm{m}, 100 \mu \mathrm{m})$; and decreased to a stress of $260 \mathrm{MPa}$ at the chip root at $(1700 \mu \mathrm{m}, 250 \mu \mathrm{m})$. Along the primary shear plane of Material Model 2, the stress at the tool tip $(0 \mu \mathrm{m}, 0 \mu \mathrm{m})$ was $340 \mathrm{MPa}$; it then increased to $390 \mathrm{MPa}$ at $(100 \mu \mathrm{m}, 50 \mu \mathrm{m})$; increased continually to a maximum of $425 \mathrm{MPa}$ at $(600 \mu \mathrm{m}, 100 \mu \mathrm{m})$; and decreased to a stress of $375 \mathrm{MPa}$ at the chip root at $(1700 \mu \mathrm{m}, 250 \mu \mathrm{m})$. The distribution of stress along the primary shear plane of Material Model 3 were consistent with the strain observations: along the primary shear plane the stresses decreased from a 
maximum of $410 \mathrm{MPa}$ at the tool tip $(0 \mu \mathrm{m}, 0 \mu \mathrm{m})$ to a minimum of $260 \mathrm{MPa}$ at the chip root $(1700 \mu \mathrm{m}, 250 \mu \mathrm{m})$. The PDZ in Material Model 1, was approximated as being contained within the stress contour of $300 \mathrm{MPa}$, which corresponded to a width of $600 \mu \mathrm{m}$ measured along a direction perpendicular to the primary shear plane. The PDZ in Material Model 2, was approximated as being contained within the stress contour of $400 \mathrm{MPa}$, which also corresponded to an approximate width of $600 \mu \mathrm{m}$ measured along a direction perpendicular to the primary shear plane. The width of the PDZ in Material Model 3, was determined by the concentration of stress contours between a stress of $230 \mathrm{MPa}$ and $330 \mathrm{MPa}$, which was measured to be approximately $600 \mu \mathrm{m}$ measured along a direction perpendicular to the primary shear plane.

The tool tip stress at $(0 \mu \mathrm{m}, 0 \mu \mathrm{m})$ in the experimental results was reported as $422 \mathrm{MPa}[56]$ and decreased along the primary shear plane to a minimum of $330 \mathrm{MPa}$ at the chip root $(1700 \mu \mathrm{m}, 250 \mu \mathrm{m})$. Material Model 3 was the only material model of those studied that accurately predicted this stress trend in the PDZ. The values of maximum stress for experimental findings correlate well to the numerical results of Material Model 3; the numerical results underestimated the experimental values by only $3 \%$.

The conclusion drawn from the correlation of numerical and experimental stress distributions is that Material Model 3 was the only material model of those studied that was capable of predicting stress distributions in close agreement with experimental findings. The stress distributions in Material Models 1 and 2 were unacceptable because they failed to recognize the increase in stresses in the SDZ, and instead falsely predicted a stress increase in the PDZ. The accuracy of Material Model 3 could be expected because the numerical stress-strain relationship was derived directly from the experimental data used for correlation.

An analysis of the Johnson-Cook equation (Equation (6.1)) pinpointed the strain rate term as the cause of numerical stress distribution inaccuracies in Material Models 1 and 2. The first term of the equation defines a simple power law between stress and strain, while the third term is neglected because thermal-mechanical analysis is not yet developed in the Eulerian formulation. Hence the only term that can contribute to the discrepancies in the stress distributions is the second term, which accounts for strain rate. The influence of strain rate on the stress distribution is discussed further in Section 10.5. 


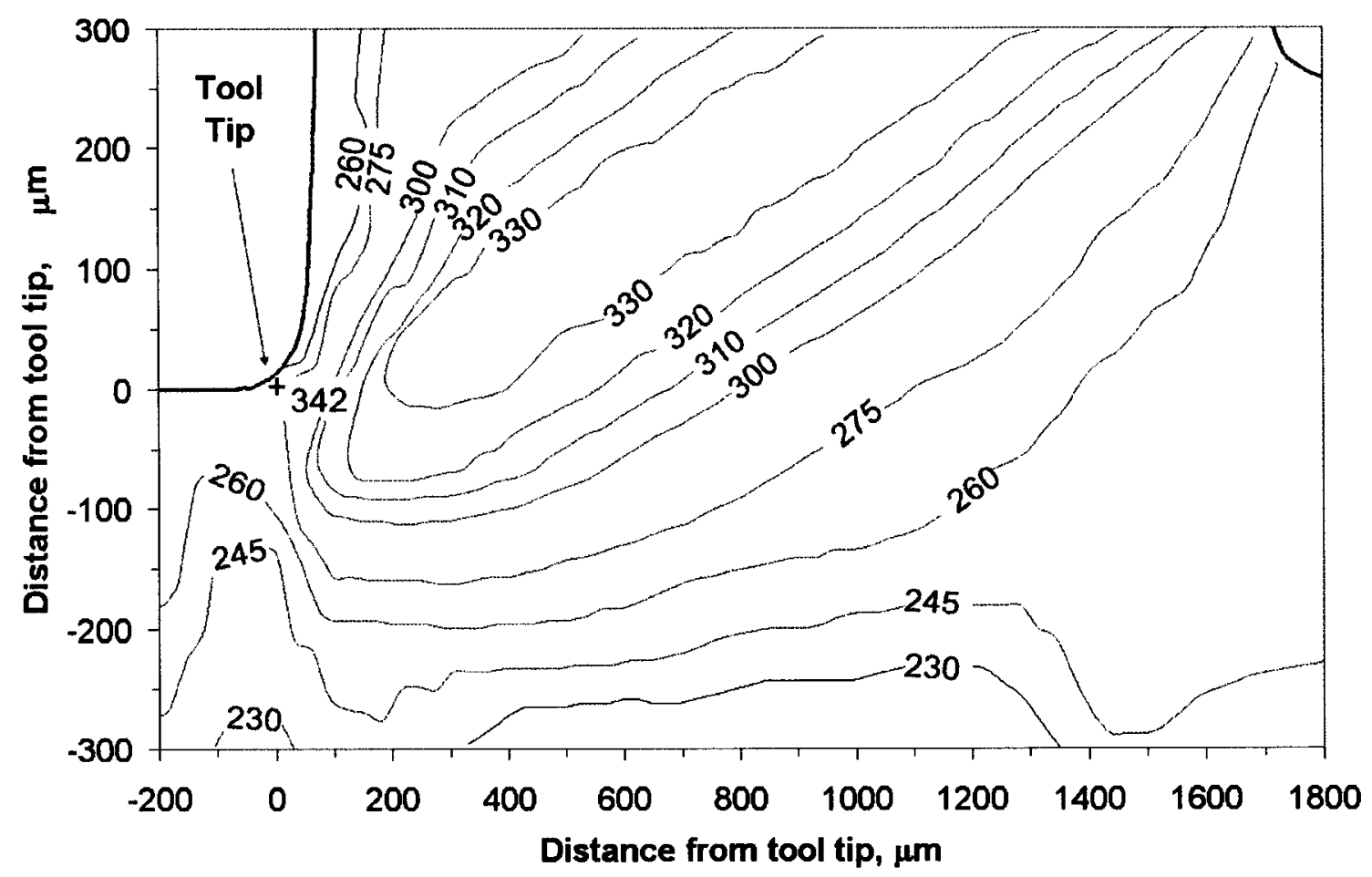

Figure 10.6: Contours of flow stress, in units of MPa, according to Material Model 1 with $\mu=0.84$ at $\mathrm{t}=\mathbf{2 0 . 0} \mathrm{ms}$.

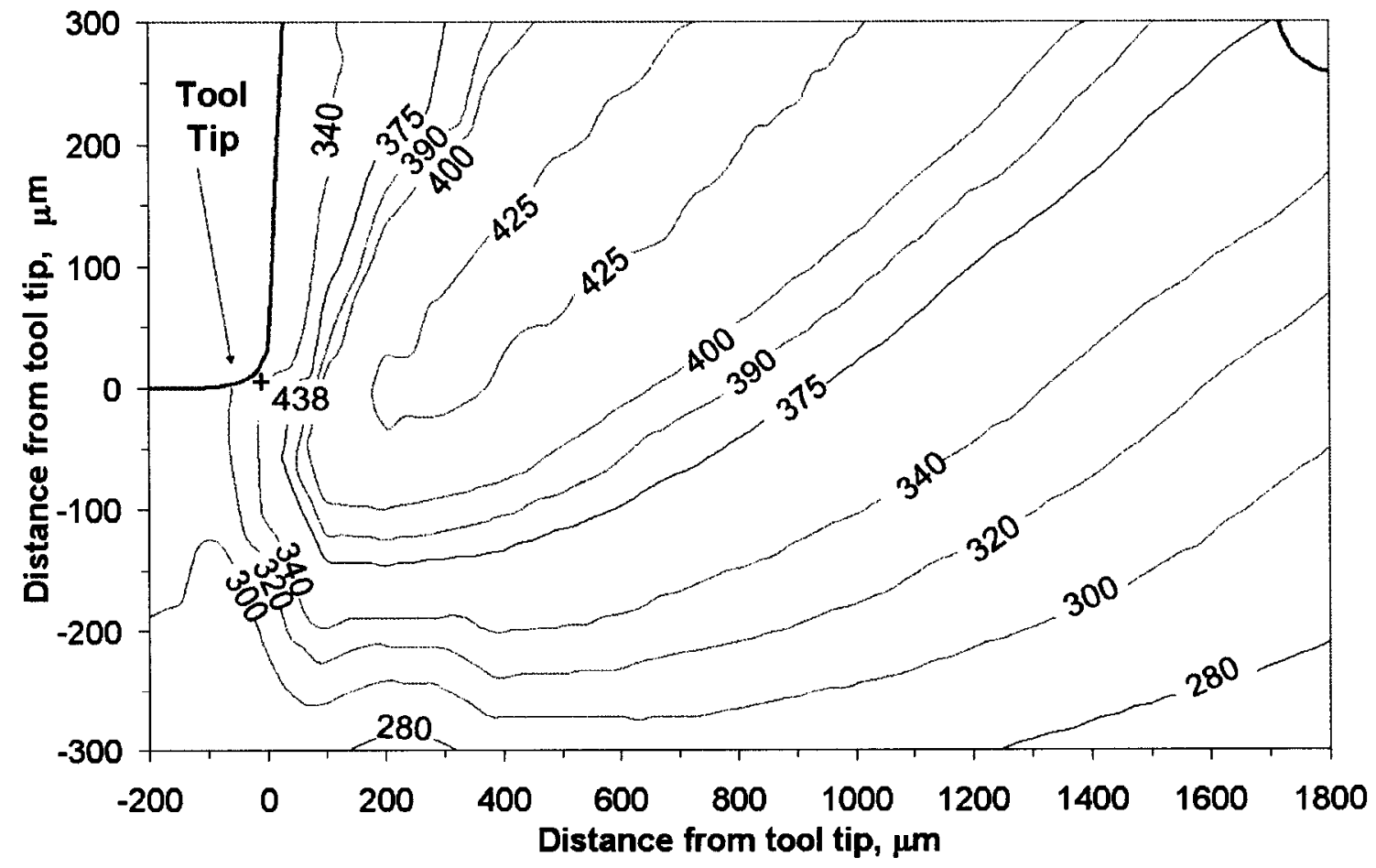

Figure 10.7: Contours of flow stress, in units of MPa, according to Material Model 2 with $\mu=0.39$ at $\mathrm{t}=20.0 \mathrm{~ms}$. 


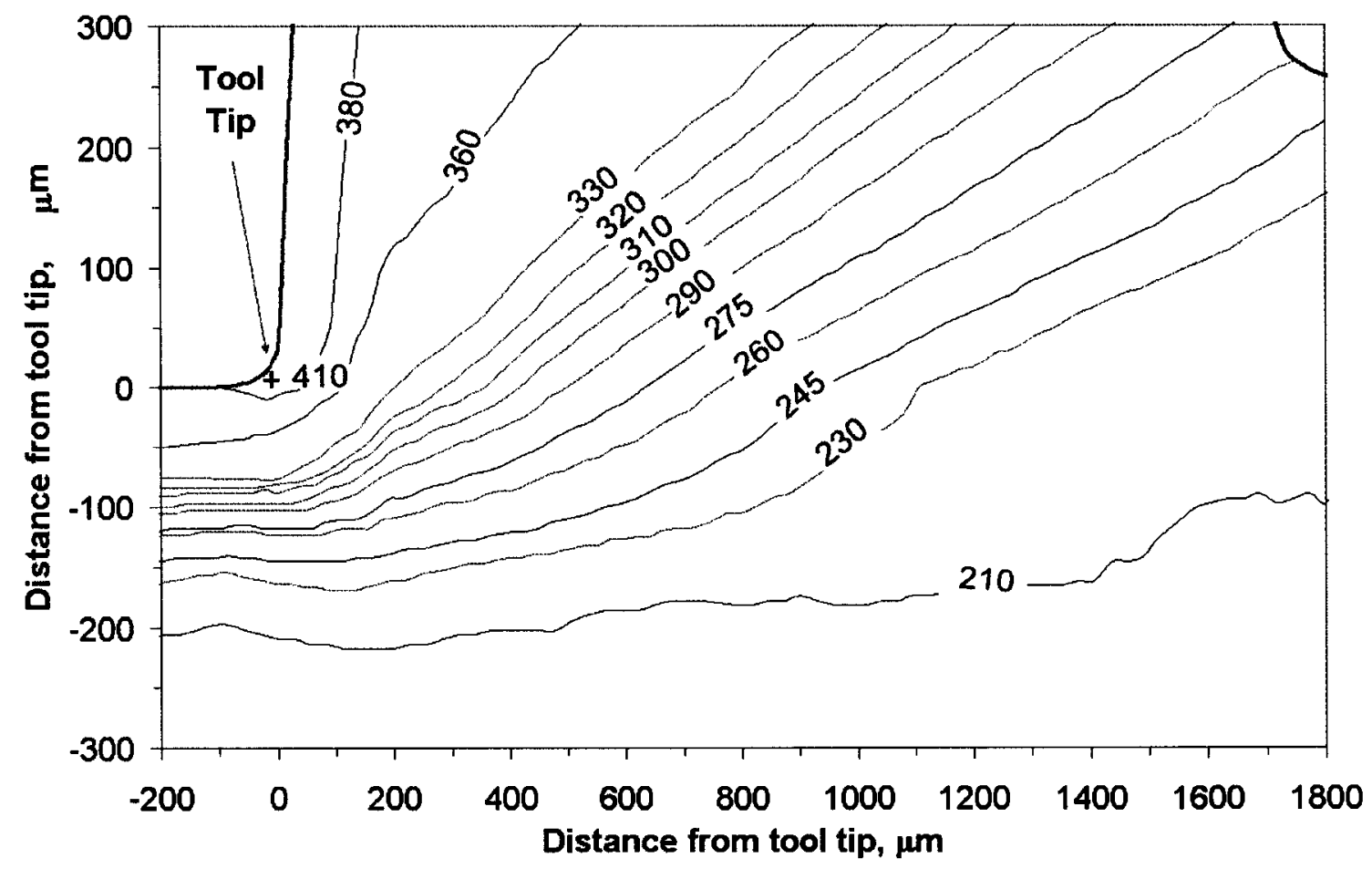

Figure 10.8: Contours of flow stress, in units of $\mathrm{MPa}$, according to Material Model 3 at $\mathrm{t}=30.0 \mathrm{~ms}$.

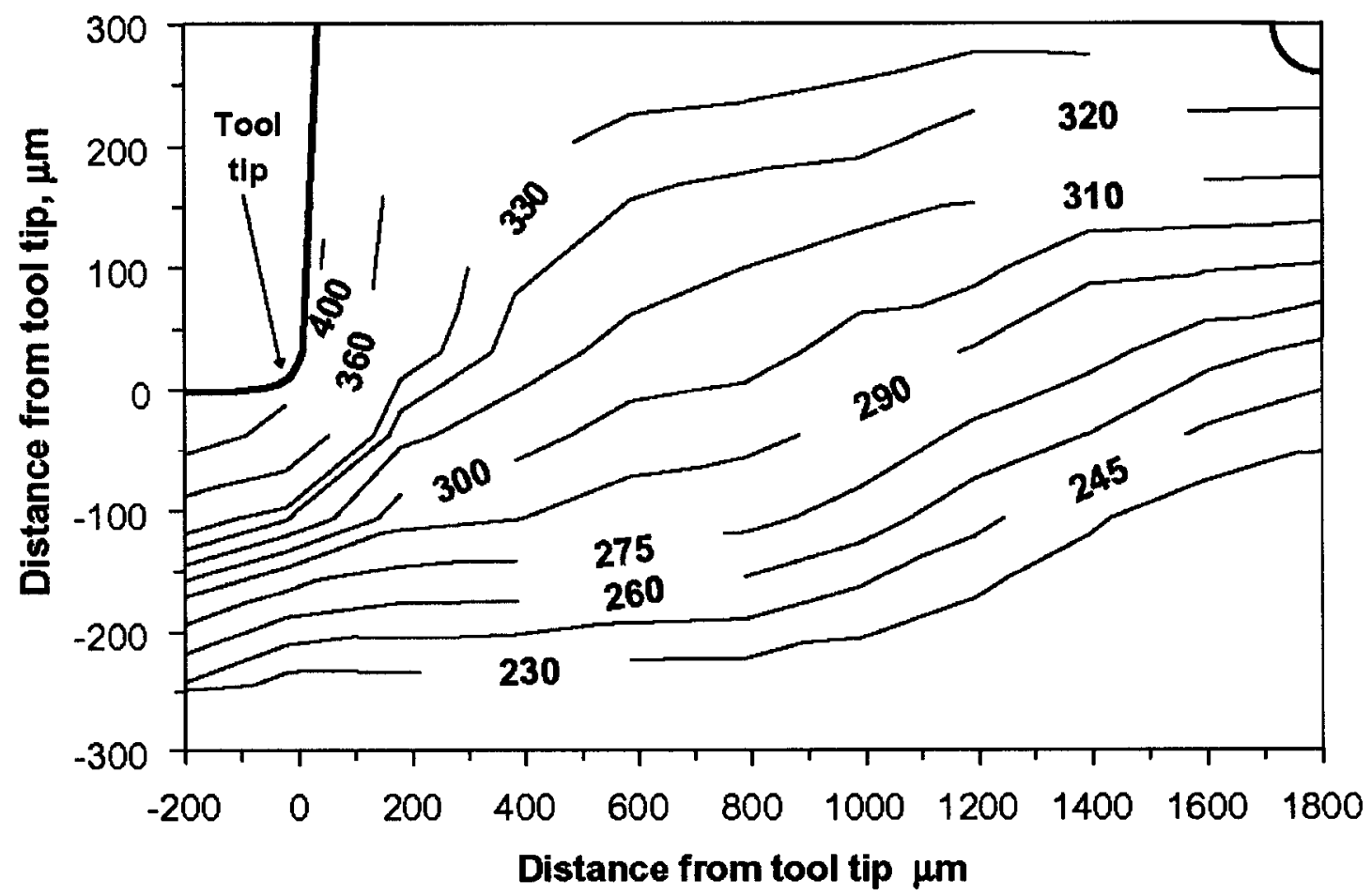

Figure 10.9: Contours of flow stress, in units of MPa, according to experimental data [56]. 


\subsubsection{External Work}

The variation of external work per unit model depth is shown in Figure 10.10 for all three material models. The graph of each of the material models ended at its designated simulation termination time, which was $20.0 \mathrm{~ms}$ for Material Models 1 and 2, and $30.0 \mathrm{~ms}$ for Material Model 3. The external work for all material models remained zero until $5.0 \mathrm{~ms}$, the time when the workpiece first contacted the tool and strain began to accumulate. Beyond $5.0 \mathrm{~ms}$ each graph was linear; the different slopes resulted in slight differences in the external work calculation. External work was analyzed at $20.0 \mathrm{~ms}$, the maximum time for which data for all three material models was available. At $20.0 \mathrm{~ms}$, Material Model 2 exhibited the highest value $\left(4.60 \mathrm{~J} \cdot \mathrm{mm}^{-1}\right.$ ), followed by Material Model $1\left(3.60 \mathrm{~J} \cdot \mathrm{mm}^{-1}\right)$, and then Material Model $3\left(2.76 \mathrm{~J} \cdot \mathrm{mm}^{-1}\right)$. The differences in the external work required to deform the workpiece material are a direct result of the strain being accumulated in each of the material models; the larger the strain that develops in the workpiece, the more work must be done to displace the material. As discussed in Section 10.3.2, Material Model 2 had the highest maximum strain of 8.3, followed by Material Model 1 with a strain of 7.5, and finally Material Model 3 with maximum strain of 5.6. The order of highest to lowest external work corresponds to the order of highest to lowest strain attained in the workpiece for each material model. The external work required for the experimental tests was not available for experimental-numerical correlation. 


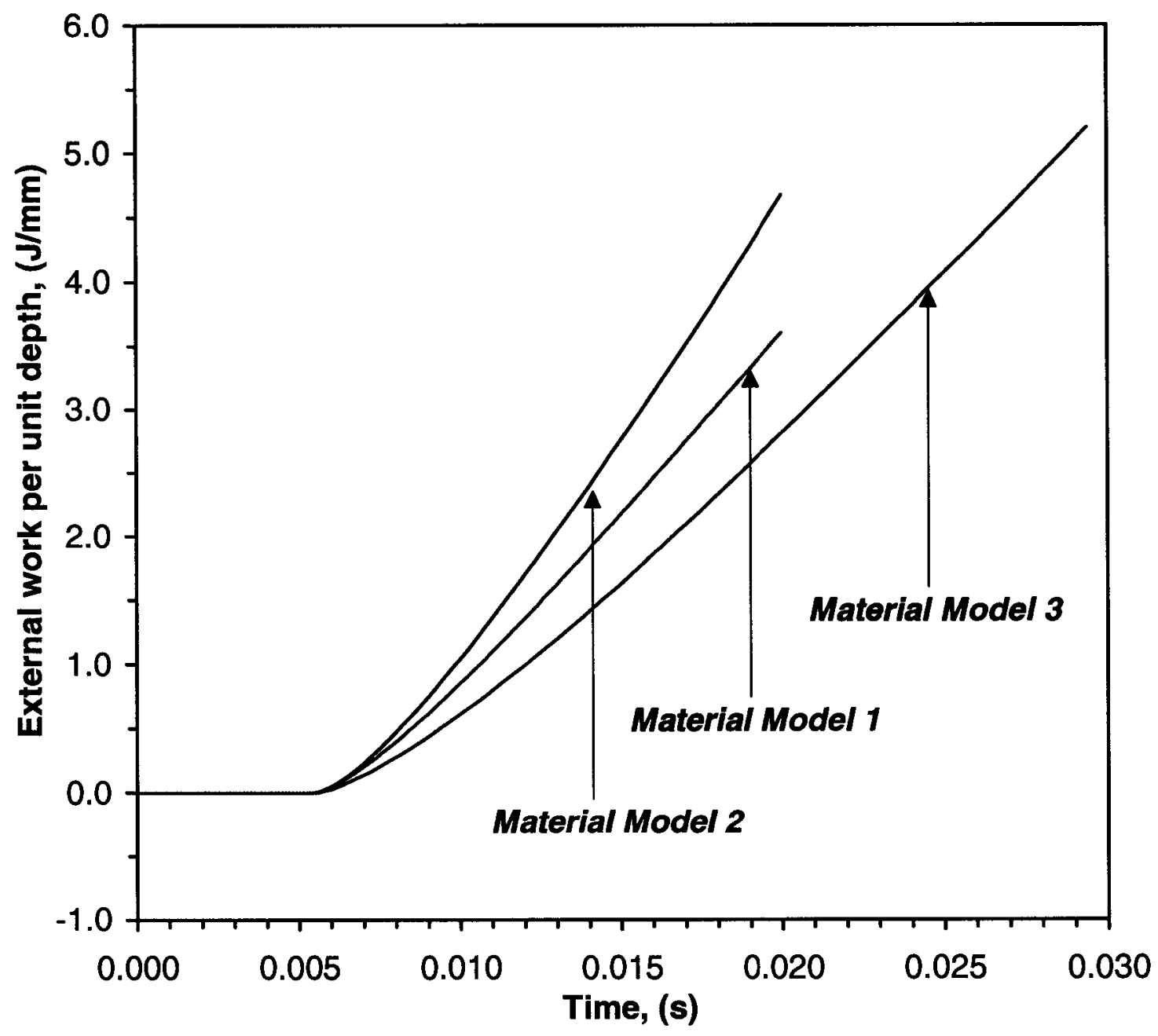

Figure 10.10: External work variation, in units of $\mathrm{J} \cdot \mathrm{mm}^{-1}$ in: Material Model 1 with $\mu=0.84$ and termination time of $20.0 \mathrm{~ms}$, Material Model 2 with $\mu=0.39$ and termination time of $20.0 \mathrm{~ms}$, and Material Model 3 with termination time of $30.0 \mathrm{~ms}$. 


\subsubsection{Forces and Power Consumption}

Cutting and thrust forces are shown in Figures 10.11 and 10.12, respectively, for the three material models. The total power consumed during the cutting operations was derived from the cutting forces via Equation (2-10) and is illustrated in Figure 10.13. The dashed line in each of the figures represents the steady state force or power values attained during the experimental machining tests. The forces and total power are those obtained with $\mu=0.84$ for Material Model 1, and $\mu=0.39$ for Material Model 2. For Material Model 3, friction was accounted for by the experimental input data.

\section{Cutting Forces $\left(F_{c}\right)$}

The cutting force for all three material models remained zero until workpiece-tool contact occurred at $5 \mathrm{~ms}$; it then rose rapidly, and slowly began to level off. At the termination time of $20.0 \mathrm{~ms}$ the cutting forces were still slightly on the rise but the chip formation, strain distribution, and stress distribution indicated that steady state conditions had been reached; in accordance to these observations the cutting force value at termination time was designated as steady state. Material Models 1 and 2 were assumed to have steady state cutting forces of $1430 \mathrm{~N}$ and $1970 \mathrm{~N}$, respectively. Material Model 3, terminated at $30.0 \mathrm{~ms}$, attained a steady state cutting force of $1332 \mathrm{~N}$.

The experimental cutting force (dashed line in Figure 10.11) had a steady state value of $1177 \mathrm{~N}$. Material Model 3 best predicted this force, with an overestimation of only $13 \%$. Material Model 2 overestimated the experimental cutting force by $21 \%$. Material Model 1 provided the least accurate cutting force approximation with an overestimation of $67 \%$.

\section{Thrust Forces $\left(F_{t}\right)$}

The trends observed in the thrust forces for all three material models resembled the cutting forces. The thrust force remained at zero until workpiece-tool contact occurred at $5.0 \mathrm{~ms}$; it then rose rapidly and slowly reached a steady state plateau. The thrust forces at the termination time of $20.0 \mathrm{~ms}$ for Material Models 1 and 2 were assumed to reflect their steady state values, which were of 962 and $941 \mathrm{~N}$, respectively. 


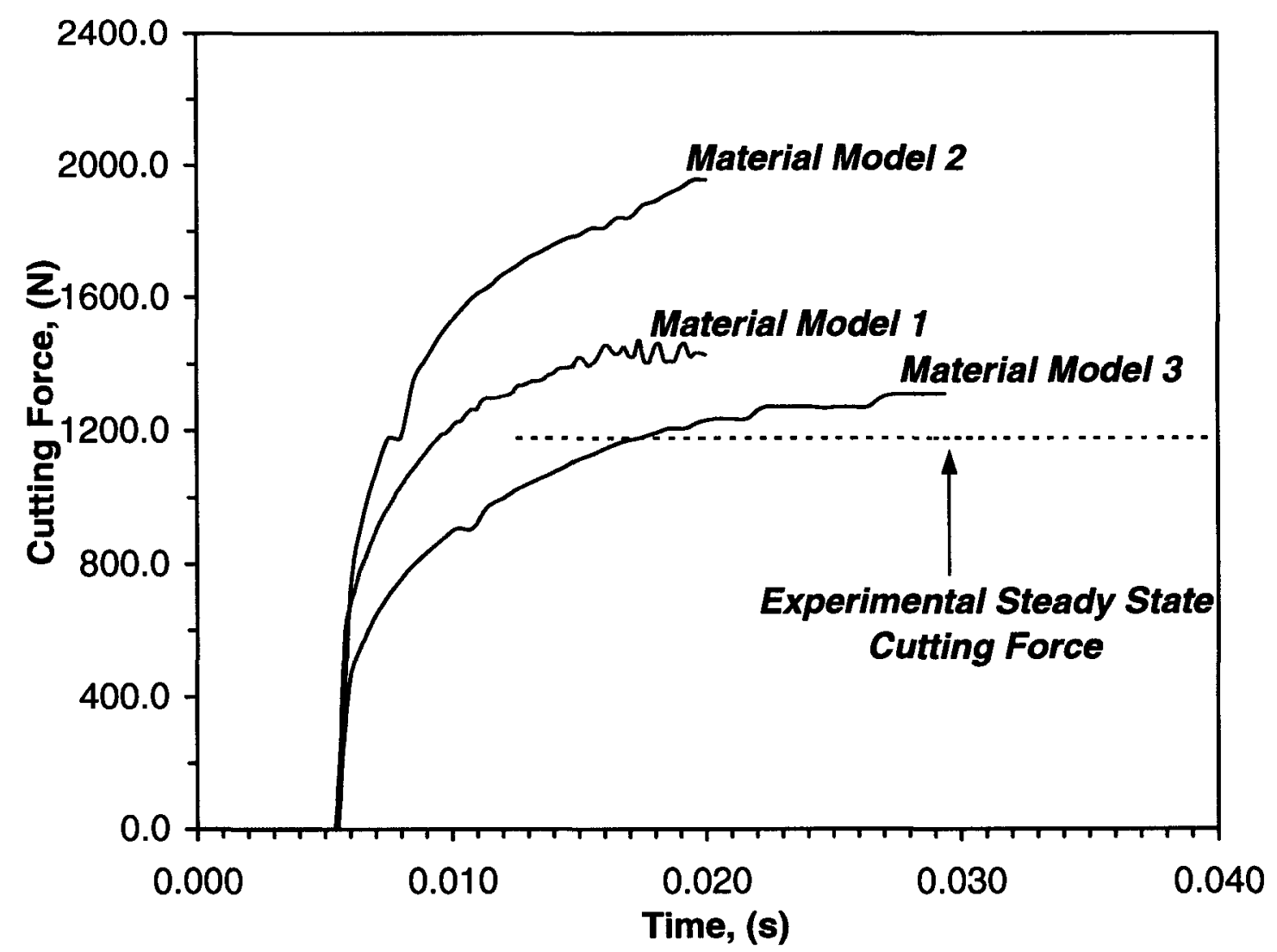

Figure 10.11: Cutting force $\left(F_{c}\right)$, in units of $\mathrm{N}$, for all three studied material models with respect to time, and the steady state cutting force for the machining tests [56]. 


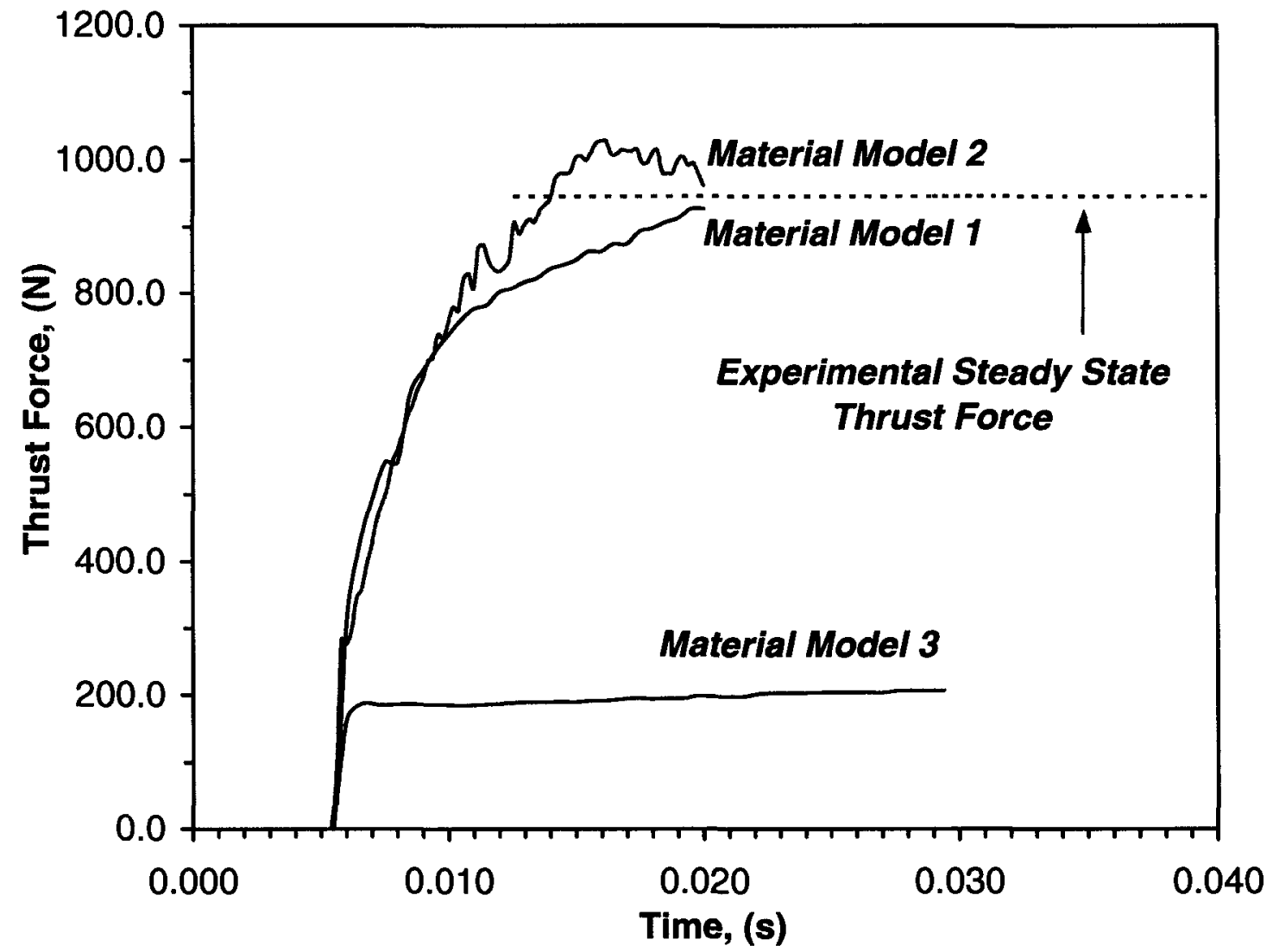

Figure 10.12: Thrust force $\left(F_{t}\right)$, in units of $\mathrm{N}$, for all three studied material models with respect to time and the steady state thrust force for the machining tests [56]. 


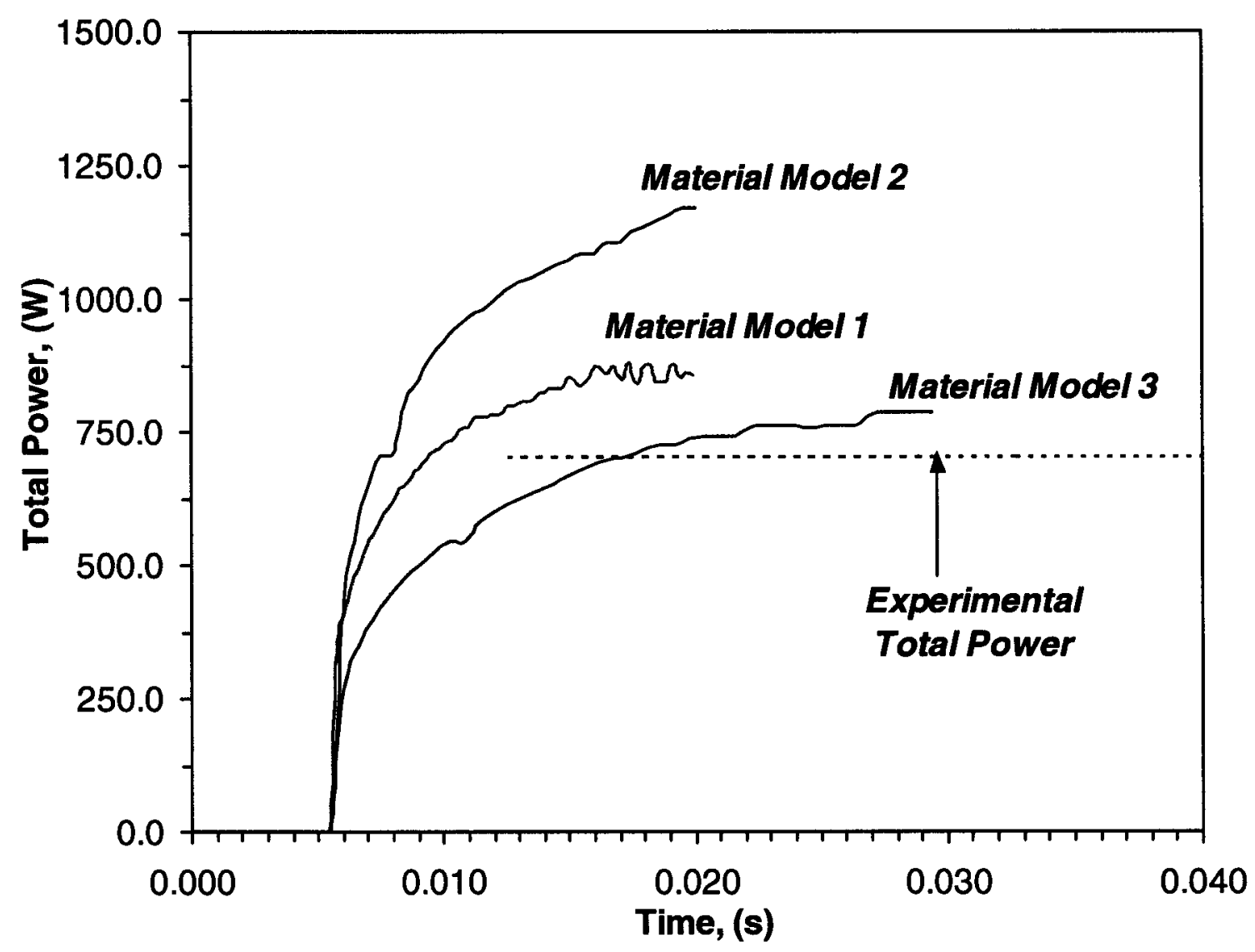

Figure 10.13: Total power $\left(P_{t}\right)$, in units of $\mathrm{W}$, for all three studied material models with respect to time, and the power consumed for the steady state of the machining tests [56]. 
Material Model 3, which terminated at $30.0 \mathrm{~ms}$, attained a steady state thrust force of $209 \mathrm{~N}$. The thrust force is a product of the cutting force and the coefficient of friction. Because friction was not applied in the contact algorithm for Material Model 3, the resultant value was well below Material Models 1 and 2 .

The experimental thrust force, marked by the dashed line in Figure 10.12, had a steady state value of $946 \mathrm{~N}$. Material Models 1 and 2 both closely predicted the experimental thrust force with variations of 2 and $1 \%$, respectively. Contrary to its close prediction of the cutting force, Material Model 3 underestimated the thrust forces by $78 \%$.

\section{Total Power Consumed $\left(P_{t}\right)$}

Figure 10.13 depicts the total power consumption of the cutting process for each material model. The power varied with time in the same manner as the cutting force, remaining zero until contact occurred between the tool and workpiece, then rising rapidly and slowly levelling off to its steady state value. The power consumed by the chip formation in Material Model 1 was $858 \mathrm{~W}$. Material Model 2 cutting simulation consumed $1182 \mathrm{~W}$ and Material Model 3 consumed $799 \mathrm{~W}$.

Power consumption during the experimental machining operation was calculated from the experimental cutting forces through Equation (2-10), and was equal to $706 \mathrm{~W}$. Because the total power consumed is proportional to the cutting force, the accuracy of numerical cutting force predictions had a direct effect on the precision of the calculated power consumption. Material Model 3 was the most accurate in predicting the cutting force; therefore it was also the most accurate in predicting the total power consumed, with a variation of $13 \%$. Material Models 2 and 3 overestimated the consumed power by $21 \%$ and $67 \%$, respectively.

The three material models are compared as follows; Material Model 3 was able to predict the cutting force and total power consumed within $13 \%$, but generated a $78 \%$ error in the thrust force estimations. Material Model 2 was capable of predicting the cutting force and power consumption only within $21 \%$ but is extremely accurate in predicting the thrust force within $1 \%$. Material Model 1 was highly inaccurate in 
predicting the cutting force and power consumption, generating errors of $67 \%$, but predicted the thrust force within 2\%. Hence Material Model 2 provides the best compromise between the prediction of cutting force and/or power consumption, and predicting thrust forces.

When choosing the best material model with respect to force and power predictions it is important to recognize that one of the objectives of machining is to minimize the required power input. Because minimizing power input translates to minimizing power consumption and thereby minimizing cutting forces, the accurate prediction of cutting forces plays a much more important role in metal cutting simlations then the accurate prediction of thrust force. As such for the numerical simulations conducted, Material Model 3 is the best model because it most precisely predicted the cutting forces and power consumption of the process. Additional work must be conducted to fully investigate the weaker area of its results, i.e., the thrust force predictions. 


\subsection{DETERMINATION OF BEST NUMERICAL MODEL}

Of the three element formulations studied in the course of this project, the Eulerian element formulation was found to most accurately model the extreme workpiece deformation which occurs at the tool tip to form a chip through shearing. Each of the three material models analyzed in the Eulerian formulation study had strong predictive capabilities in one or more of the following areas: chip geometry, strain and stress distribution, cutting and thrust forces, and power consumption. However, the best material model should have an ability to predict all the aspects of chip formation to a high degree.

It was concluded that experimental chip geometry can be replicated by all of the material models, but notice was taken of the fact that Material Models 1 and 2 required friction coefficient manipulation, but Material Model 3 did not. Because the strain distribution is independent of the material model, it was expected to be similar for all cases with the exception of the SDZ, where the friction applied in Material Models 1 and 2 caused a slight rise in strains. Material Models 1 and 2 were severely deficient in the prediction of stress distribution. The models predicted a concentration of stress in the PDZ and softening in the SDZ, which is in disagreement with the strain distribution in experimental findings. Material Model 3 was the only material that accurately predicted the distribution of stress with increased stresses in the SDZ instead of the PDZ. In addition to predicting the correct stress distribution, Material Model 3 was also the most precise model for values of stress.

The experimental cutting forces and power consumption best correlated to Material Model 3; Material Models 1 and 2 most accurately predicted the thrust force. Because the cutting force and power consumption are points of interest when analyzing a metal cutting operation, Material Model 3 is the best material when considering forces.

Material Model 3 employing an Eulerian element formulation was considered the best numerical model of those studied in this research. The material model boasts an ability to predict as accurate chip geometry without fine-tuning the coefficient of friction to fit experimental results. It is also superior to the other two models in its correlation 
with the experimental stress distribution. Material Model 3 gives an accurate portrayal of the strain distribution and strain values in the material ahead of the tool tip, and closely predicts the cutting forces and power consumption during the cutting process. One drawback of this material model is that the stress-strain behaviour must be obtained from extensive experimental investigations; as such, experimental machining tests must be performed and analyzed prior to any numerical investigation thus defeating the purpose of FE modeling. This drawback can be overcome by generating and making available a library of Voce equation parameters to be referenced when modeling the metal cutting of various materials.

\subsection{COMPUTATION OF STRAIN RATES}

Experimental strain rate distribution data was not available in literature but was beneficial for correlation with simulation. A procedure was developed to calculate strain rate distributions in the PDZ in the material ahead of the tool tip, based on an assumption that during dynamic deformation any flow line, as depicted by inclined grain boundaries, will take the shape of a consecutive flow line within an increment of time (Figure 10.14). Considering two successive flow lines, the strain rates in the PDZ can be estimated using Equation (10-1).

$$
\dot{\varepsilon}=\frac{d \varepsilon}{d t}
$$

where $d \varepsilon$ is the strain increment between the flow lines and $d t$ is the particular increment of time required for the first flow line to occupy the position of the second. The time increment is determined from the velocity of the material and the distance between the portions of the consecutive flow lines that are parallel. The first region, designated as $\mathrm{A}$, includes all areas under the upper boundary of the primary shear zone, in which the material velocity is assumed to be equivalent to the cutting velocity. Strain rates are calculated in region $\mathrm{A}$ by Equation (10-2).

$$
\dot{\varepsilon}_{A}=\frac{\partial \varepsilon_{A}}{\partial x} v_{c}
$$


where $\partial x$ and $\partial \varepsilon_{A}$ are the distance and strain increments, respectively, calculated along lines parallel to the direction of cutting velocity, as shown in Figure 10.14. The second region, designated as $B$, includes the chip above the primary shear zone where the material moves along a direction parallel to the rake face with the velocity of the chip. In this region, the strain rates are calculated by Equation (10-3).

$$
\dot{\varepsilon}_{B}=\frac{\partial \varepsilon_{B}}{\partial u} v_{c h i p}
$$

where $v_{\text {chip }}$ is the chip velocity, and $\partial u$ and $\partial \varepsilon_{B}$ are the distance and strain increments, respectively, calculated along lines parallel to the direction of chip velocity.

The equivalent strain rates found experimentally are presented in Figure 10.15 (a), while numerical equivalent strain rates generated by the Eulerian workpiece modeled with Material Model 3 are shown in Figure 10.15 (b). The maximum strain rate occurred at the tool tip reaching values of approximately $6100 \mathrm{~s}^{-1}$ and $3500 \mathrm{~s}^{-1}$ for the experimental and numerical results, respectively. The strain rates increased from a maximum at the tool tip to approximately $1750 \mathrm{~s}^{-1}$ and $1500 \mathrm{~s}^{-1}$ at $(200 \mu \mathrm{m}, 0 \mu \mathrm{m})$ in the numerical and experimental results, respectively. Below the cutting line, the strain rates decreased to $150 \mathrm{~s}^{-1}$ at $(0,-200 \mu \mathrm{m})$ and beyond this depth they were negligible. The contour plots of strain rates clearly showed that the material displacement is contained within the PDZ since large strain rates were concentrated in this region. The strain rates in the PDZ were lower then those detected at the tool tip; they reached values of $500 \mathrm{~s}^{-1}$ and $1000 \mathrm{~s}^{-1}$ in the experimental and numerical findings, respectively.

Although the steps taken to calculate the experimental strain rates may be an area of further discussion, the agreement of the experimental strain rate distribution with the order of the strain rate values found in the validated FE model provides support to the calculation. The suppression of strain rates within the PDZ also supports a statement made in Section 10.3.2 regarding the cause of stress increase in the PDZ of Material Models 1 and 2. In that statement, the increase was attributed to the strain rates. From Figure 10.15, it is clear that high strain rates are contained within the PDZ and have contributed to increasing the stresses in this region as calculated by the material model. 
Therefore, the causes of the poor stress distribution predictions of the Johnson-Cook material model are identified in 1) the consideration of strain rate effects, and 2) the omission of the thermal softening influence. 


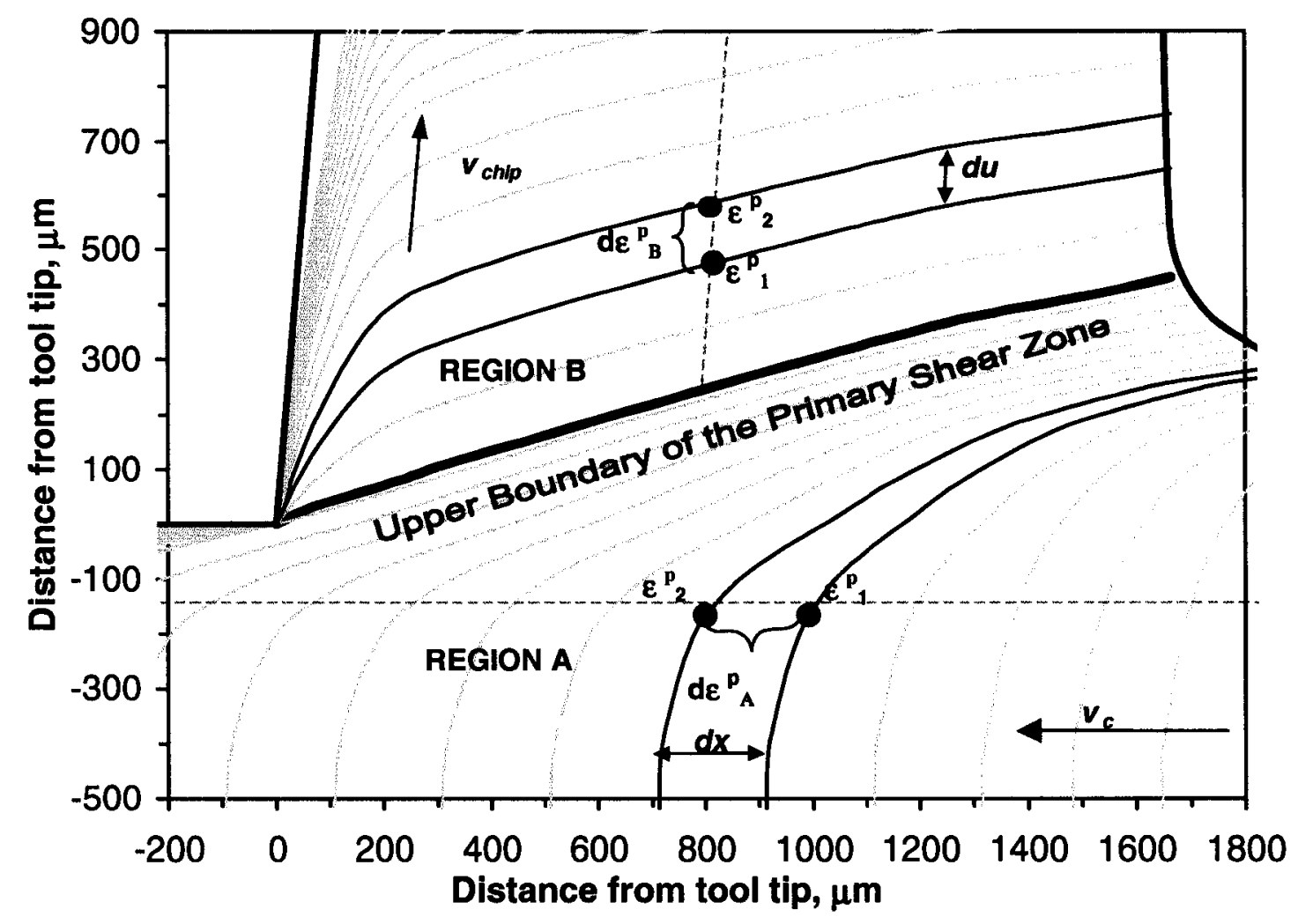

Figure 10.14: Variables defined in the experimental analysis of strain rate distributions in the material ahead of the tool tip. 


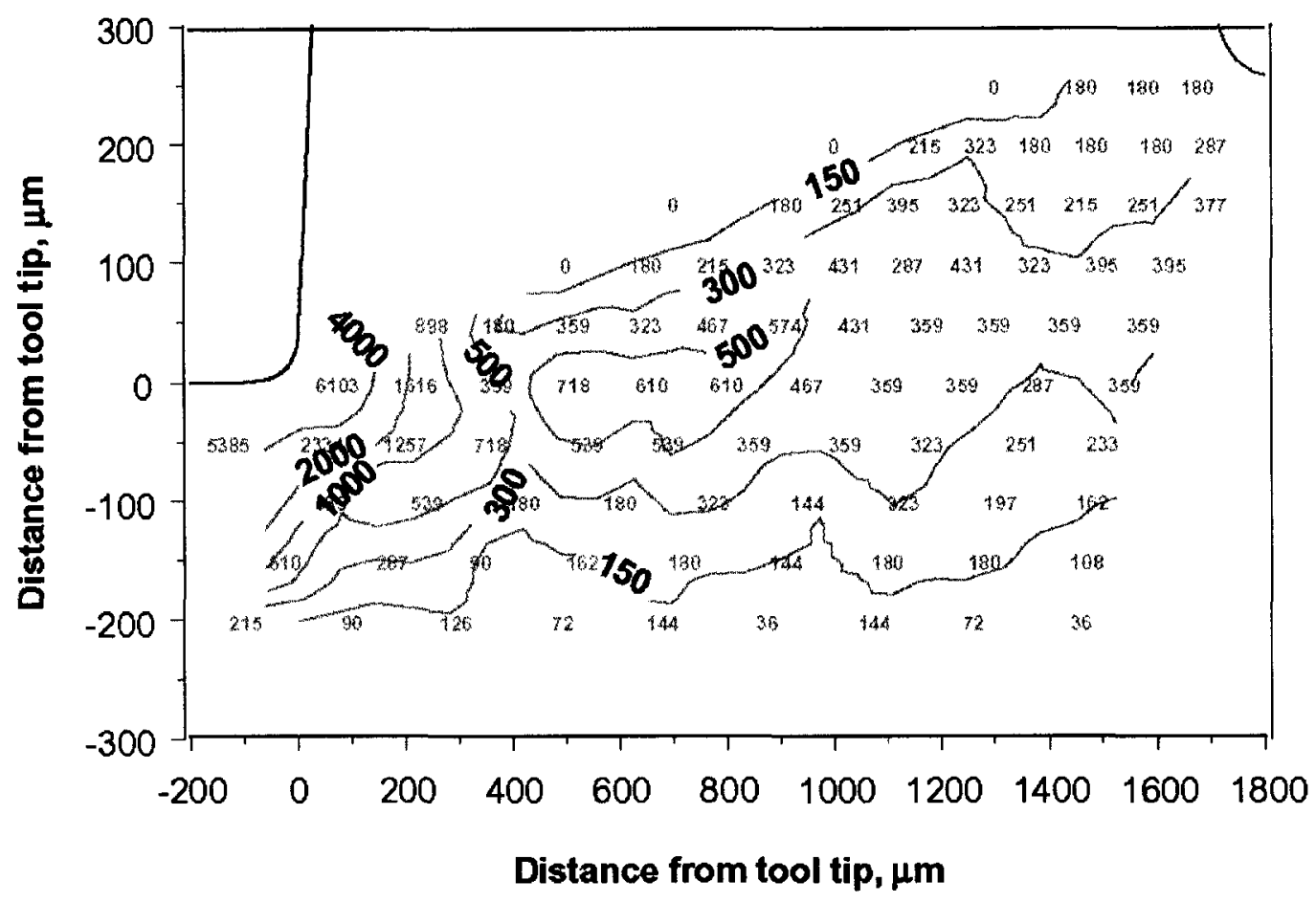

(a)

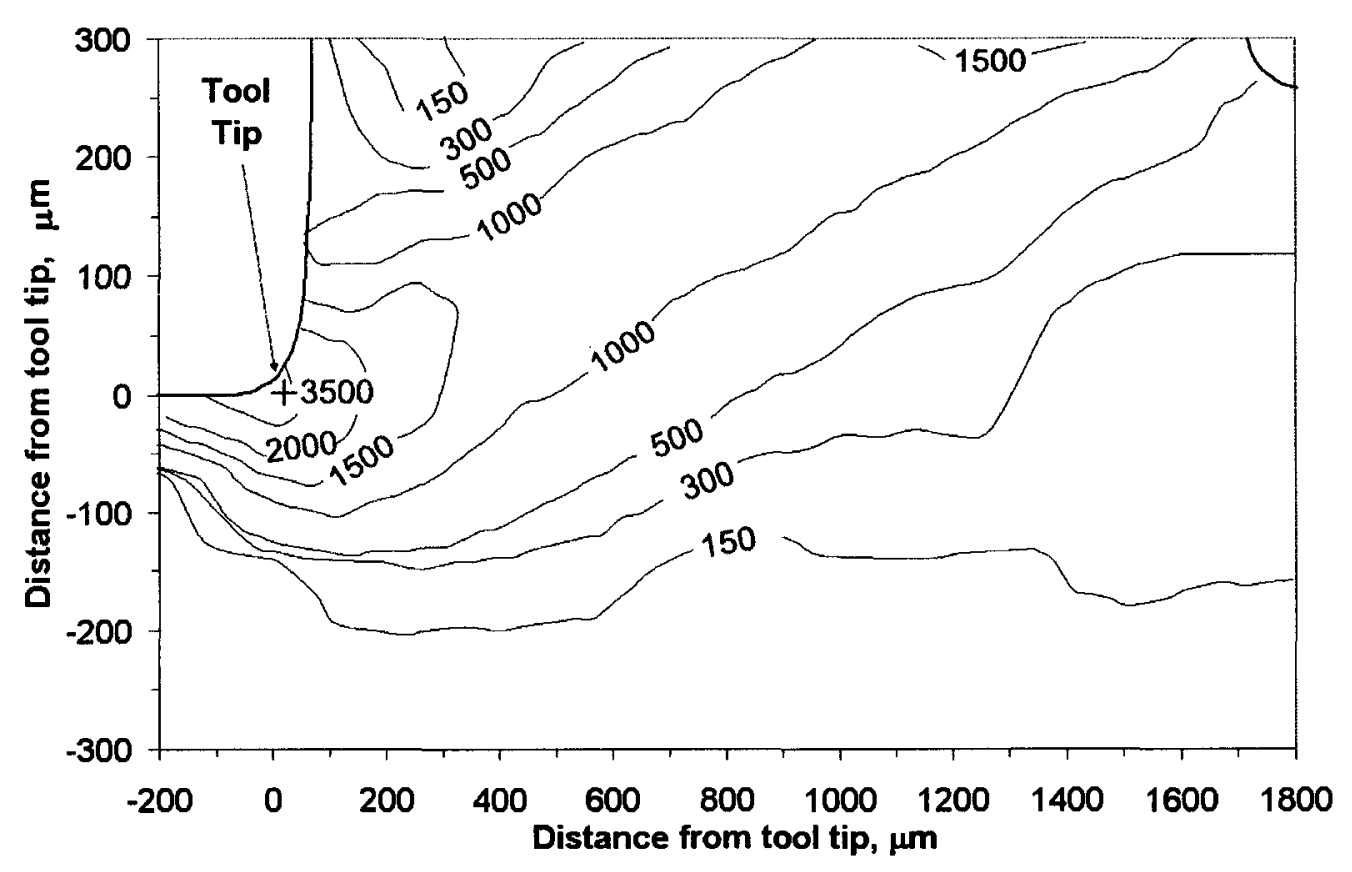

(b)

Figure 10.15: Strain rates, in units of $\mathrm{s}^{-1}$, according to (a) experimental findings, and (b) FE results with workpiece modeled by Material Model 3. 


\section{CHAPTER 11}

\section{Conclusions}

The presented study has focused on the development of a FE model for the orthogonal metal cutting of commercial purity ETP copper. Three element formulations, including the Lagrangian, ALE, and Eulerian element formulations, have been investigated in the scope of this project. Conclusions drawn from the presented experimental and numerical observations are given below:

\section{Lagrangian Element Formulation}

1. A model was developed for the steady state chip formation during metal cutting, using the Lagrangian element formulation. Results revealed that most significant mesh distortion occurred in the proximity of the tool tip. The mesh experienced severe deformation during the modeling of metal cutting because the mesh was attached to the material, which was being deformed most drastically at the tool tip. The finer the elements in this area, the more excessive the distortion became.

2. Mesh adaptivity was applied to the Lagrangian workpiece, to reduce the extent of mesh distortion occurring at the tool tip. Applying mesh adaptivity considerably reduced the distortion at the tool tip, but because a whole new mesh was generated during mesh adaptivity in LS-DYNA, nodal constraints originally placed on the workpiece were lost. Therefore, mesh adaptivity was not effective in modeling of metal cutting using the LS-DYNA software.

\section{ALE Element Formulation}

3. A model was developed for the steady state chip formation using the ALE element formulation. Results showed definite improvements over the Lagrangian technology in reducing mesh distortion; however, with successive smoothing the mesh was still incapable of modeling the large material deformation at the tool tip without influencing the constraints initially placed on the workpiece. The model was not successful in the modeling of chip formation during metal cutting. 
4. The ALE element formulation was also used in the modeling of incipient chip formation. Despite several attempts to optimize the smoothing mechanisms, the simulations terminated erroneously due to the severe mesh distortion occurring in the proximity of the tool tip. Thus this model was also unsuccessful in simulating material deformation during machining.

\section{Eulerian Element Formulation}

5. A FE model for the orthogonal cutting process was developed using an Eulerian element formulation, and was the first documented attempt to use this formulation in modeling of machining. The numerical model employing the Eulerian element formulation for modeling of the workpiece was found to most accurately predict the deformation state of the workpiece material undergoing the metal cutting operation. The workpiece was modeled using three distinct material models, two of which were based on the Johnson-Cook constitutive equation and the third was an elastic plastic hydrodynamic constitutive model.

6. The numerical results for each of the material models applied, were correlated to experimental findings reported in literature on the basis of: chip geometry, strain distribution, stress distribution, cutting and thrust forces, and power consumed by the operation. Each of the material models were capable of reproducing the chip geometry of the experimental machining samples; to do so, the Johnson-Cook material models (Material Models 1 and 2) required the optimization of the contact friction, while Material Model 3 inherently included friction and reproduced the correct chip thickness without assigning interface friction in the contact algorithm. All of the material models well predicted the strain distributions in the material ahead of the tool tip. Material Model 3 was the most accurate in predicting the stress distribution, cutting forces and power consumption. Material Models 1 and 2 (those represented by the Johnson-Cook constitutive equation) were especially inaccurate in their predictions of stress distributions, finding that increased stresses were concentrated in the PDZ, while experimentally they increased in the SDZ. 
7. Material Model 3, the hydrodynamic material model based on an experimentally determined Voce type stress-strain relationship, was found be the most accurate of all tested material models for predicting the chip geometry, stress, strain, cutting force and power consumption found through experimental observations. Correlations between the numerical and experimental findings, which validated the precision of the developed model, are summarized below:

- The experimental chip thickness of $1.7 \mathrm{~mm}$ was nearly reproduced by the numerical model, which generated a chip with a thickness of $1.6 \mathrm{~mm}$.

- The overall trends in numerical strain and stress distribution were in good agreement with experimental observations. The maximum numerical strain of 5.6 and maximum numerical stress of $410 \mathrm{MPa}$ were located at the tool tip, which correlated well with the maximum experimental strain and stress of 8.1 and $422 \mathrm{MPa}$, respectively, also located in the tool tip region. The experimentally determined strain in the material ahead of the tool tip at $(50 \mu \mathrm{m}, 0 \mu \mathrm{m})$ was $\bar{\varepsilon}^{p}=3.64$, which compared well with $\overline{\mathcal{E}}^{p}=3.50$ calculated numerically for the same location. Also, at a distance of $50 \mu \mathrm{m}$ ahead of the tool tip, the experimentally estimated stress value was $360 \mathrm{MPa}$, which was in agreement with the numerically predicted value of $380 \mathrm{MPa}$.

- The sizes of both the primary deformation zone $(600 \mu \mathrm{m})$ and the secondary deformation zone $(80 \mu \mathrm{m})$ predicted by the hydrodynamic material model were in agreement with the experimental observations.

- The numerically determined cutting force $(1332 \mathrm{~N})$ and total power consumption $(799 \mathrm{~W})$ predicted their experimental counterparts to within $13 \%$.

8. With the support of the validated numerical results, a procedure was developed for the calculation of experimental strain rates. Both numerical predictions and experimental calculations showed that non-zero strain rates are contained within the PDZ where shearing of the material occurs during chip formation. The order of magnitude of the numerical results was in agreement with the strain rates obtained from experimental observations. 
Being the first documented model of metal cutting to utilize the Eulerian element formulation, the developed $\mathrm{FE}$ model has shown to be very innovative. It has served to accurately predict the deformation of copper subjected to metal cutting operations, and has the potential to expand in predicting the behaviour of other materials undergoing machining. The model has the capabilities of accurately predicting the cutting forces, which can estimate the power required to perform the operation. The accuracy of stress and strain distribution can also be beneficial when estimating the quality of the machined surface. The numerical model enables testing of various cutting parameters to forecast the ability of the process to fulfill functional requirement, while maintaining efficiency.

The investigations performed in this work have significantly contributed to the area of research involving FE modeling of machining, and have established that the Eulerian element formulation can be advantageous to the FE modeling of high deformation processes. 


\section{CHAPTER 12}

\section{Suggestions for Future Work}

The short-comings of the developed model are mainly caused by lack of Eulerian formulation development within the LS-DYNA Software. Thermal-mechanical coupling is currently not functional in this formulation and temperature distributions cannot be calculated, and cannot be correlated with experimental results. Since temperature has a large influence on the tool life and quality of the machined surface, it is imperative that in the future the thermal effects are incorporated into the FE model.

One of the conclusions drawn was that Material Model 3, the elastic plastic hydrodynamic material derived from an experimental flow curve, inherently incorporates the effects of friction at the tool-workpiece interface. This phenomenon should be further investigated by studying the effect of varying input variables and addition of lubrication, on the experimental Voce-type flow curve and the numerical / experimental correlations.

The established model should be validated further by performing cutting simulations of other metals including various alloys and metal-matrix composites, and correlating these results to available experimental findings. Currently, the developed model is only capable of modeling continuous chip flow, which is another limitation of the Eulerian element formulation. With further development of the software potential lies in advancing the model to enable accurate predictions of discontinuous chip flow.

The model developed in the scope of this project simulated the simplest of all machining operations, which is one tool pass of a turning operation. Multiple tool passes are frequently required to attain the desired shape and thus the model should reflect that; several cuts into one workpiece should be consecutively modeled, enabling the study of the residual stress effect on multiple pass operations. Since most metal shaping operations involve the formation of metal chips, the developed model for chip formation in metals can also act as a stepping-stone to modeling of much more complex operations, such as drilling.

The Eulerian element formulation has proven to have great potential in the modeling of high strain and strain rate processes. Its capabilities should be fully utilized in modeling other high deformation process beyond machining operations. 


\section{REFERENCES}

[1] Machinery's Handbook, 25 ${ }^{\text {th }}$ edition, Industrial Press, New York, NY, 1996.

[2] M. C. Shaw, Metal Cutting Principles, Oxford Science Publications, New York, NY, 1989.

[3] ASM Handbook, Volume 16: Metals Handbook, ASM International, Metals Park, $\mathrm{OH}, 1989$.

[4] B. F. von Turkovich, Shear Stress in Metal Cutting, J. Eng. Ind., 1970, vol. 92, pp. 151-157.

[5] P. L. B. Oxley and M. G. Stevenson, Measuring Stress/Strain Properties at Very High Strain Rates Using a Machining Test, J. Inst. Met., 1967, vol. 95, pp. 308-313.

[6] ASM Handbook, Volume 18: Friction, Lubrication, and Wear Technology, ASM International, Metals Park, OH, 1989.

[7] M. E. Merchant, Mechanics of the Metal Cutting Process: I. Orthogonal Cutting and a Type 2 Chip, J. Appl. Phys., 1945, vol. 16, pp. 267-275.

[8] V. Piispanen, Theory of Formation of Metal Chips, J. Appl. Phys., 1948, vol. 19, pp. 876-881.

[9] M. E. Merchant, Mechanics of the Metal Cutting Process: II. Plasticity Conditions in Orthogonal Cutting, J. Appl. Phys., 1945, vol. 16, pp. 318-324.

[10] E. H. Lee and B. W. Shaffer, The Theory of Plasticity Applied to a Problem of Machining, J. of Appl. Mech. Trans. ASME, 1951, vol. 18, pp. 405-413.

[11] M. C. Shaw, N. H. Cook, and I. Finnie, The Shear-Angle Relationship in Metal Cutting, Trans. ASME, 1953, vol. 75, pp. 273-283.

[12] G. W. Rowe and P. T. Spick, A New Approach to Determination of the Shear-Plane Angle in Machining, J. Eng. Ind., 1967, vol. 89, pp. 530-538.

[13] P. K. Wright, Predicting the Shear Plane Angle in Machining From Workmaterial Strain-Hardening Characteristics, J. Eng. Ind., 1982, vol. 104, pp. 285-292.

[14] R. F. Recht, Catastrophic Thermoplastic Shear, J. Appl. Mech. Trans. ASME, 1964, vol. 31, pp. 189-193.

[15] K. Okushima and K. Hitomi, An Analysis of the Mechanism of Orthogonal Cutting and its Application to Discontinuous Chip Formation, J. Eng. Ind., 1961, vol. 83 , pp. 545-556. 
[16] J. T. Black, On the Fundamental Mechanism of Large Strain Plastic Deformation: Electron Microscopy of Metal Cutting Chips, J. Eng. Ind., 1971, vol. 93, pp. 507-526.

[17] S. Ramalingam and J. T. Black, An Electron Microscopy Study of Chip Formation, Metall. Trans., 1973, vol. 4, pp. 1103-1112.

[18] J. C. Lemaire and W. A. Backofen, Adiabatic Instability in the Orthogonal Cutting of Steel, Metall. Trans., 1973, vol. 3, pp. 477-481.

[19] S. Ramalingam and J. T. Black, On the Metal Physical Considerations in the Machining of Metals, J. Eng. Ind., 1972, vol. 94, pp. 1215-1224.

[20] H. Ernst and M. E. Merchant, Chip Formation, Friction, and High Quality Machined Surfaces, Surf. Treat. Met. Trans. ASM, 1941, vol. 29, pp. 299-378.

[21] G. Dieter, Mechanical Metallurgy, McGraw-Hill, New York, NY, 1986.

[22] O. C. Zienkiewicz and R. L. Taylor, Finite Element Methods, $4^{\text {th }}$ Edition, McGraw-Hill Book Company Europe, Berkshire, England, 1994.

[23] T. Belytschko, W. K. Liu and B. Moran, Nonlinear Finite Elements for Continua and Structures, John Wiley and Sons Ltd., West Sussex, England, 2000.

[24] Introduction to Finite Element Analysis, Lecture Notes by N. G. Zamani and W. J. Altenhof, FEA Connection, 2003.

[25] O. Axelsson, Iterative Solution Methods, Cambridge University Press, New York, NY, 1994.

[26] N. M. Newmark, A Method of Computation for Structural Dynamics, J. Eng. Mech. Div. ASCE, 1959, vol. 85, pp. 67-94.

[27] LS-DYNA Theoretical Manual, Livermore Software Technology Corporation, Livermore, CA, 1998.

[28] B. Van Leer, Towards the Ultimate Conservative Difference Scheme: IV. A New Approach to Numerical Convection, J. Comput. Phys., 1977, vol. 23, pp. 276-299.

[29] B. Van Leer, Towards the Ultimate Conservative Difference Scheme: II. Monotonicity and Conservation Combined in a Second-Order Scheme, J. Comput. Phys., 1973, vol. 14, pp. 361-370.

[30] LS-DYNA 970 Keyword User's Manual Volume I, Livermore Software Technology Corporation, Livermore, CA, 2003.

[31] M. A. Meyers, Dynamic Behaviour of Materials, John Wiley \& Sons, Inc., New York, NY, 1994.

[32] LS-DYNA 970 Keyword User's Manual Volume II, Livermore Software Technology Corporation, Livermore, CA, 2003. 
[33] B. E. Klamecki, Incipient Chip Formation in Metal Cutting - A Three Dimensional Finite Element Analysis, Ph.D. Dissertation, University of Illinois, Urbana-Champaign, IL, 1973.

[34] M. P. Lajczok, A Study of Some Aspects of Metal Machining Using Finite Element Methods, Ph.D. Dissertation, North Carolina State University, Raleigh, NC, 1980.

[35] J. S. Strenkowski and K. J. Moon, Finite Element Prediction of Chip Geometry and Tool/Workpiece Temperature Distributions in Orthogonal Metal Cutting, J. Eng. Ind., 1990, vol. 112, pp. 313-318.

[36] J. T. Carroll and J. S. Strenkowski, Finite Element Models of Orthogonal Cutting with Application to Single Point Diamond Turning, Int. J. Mesh. Sc., 1988, vol. 30, pp. 899-920.

[37] T. Shirakashi and E. Usui, Simulation Analysis of Orthogonal Metal Cutting Mechanisms, Proceedings of the International Conference on Product Engineering, Tokyo, Japan, 1974, pp. 535-540.

[38] J. S. Strenkowki and J. T. Carroll, A Finite Element Model of Orthogonal Metal Cutting, J. Eng. Ind., 1985, vol. 107, pp. 349-354.

[39] A. Shih, S. Chandrasekar, and H. Yang, Finite Element Simulation of Metal Cutting Process with Strain-Rate and Temperature Effects, ASME-PED, 1990, vol. 43 , pp. 11-24.

[40] K. Iwata, K. Osakada, and Y. Terasaka, Process Modeling of Orthogonal Cutting by the Rigid-Plastic Finite Element Method, J. Eng. Mat. Tech., 1984, vol. 106, pp. 132-138.

[41] K. Komvopoulos and S. A. Erpenbeck, Finite Element Modeling of Orthogonal Metal Cutting, J. Eng. Ind., 1991, vol. 113, pp. 253-267.

[42] Z. Lin and S. Lo, J. Ultra-Precision Orthogonal Cutting Simulation for Oxygen-Free High-Conductivity Copper, Mat. Proc. Tech., 1997, vol. 65, pp. 281-291.

[43] Z. Lin and S. Lo, An Investigation of Sticking Behavior on the Chip-Tool Interface Using Thermo-Elastic-Plastic Finite Element Method, J. Mat. Proc. Tech., 2002, vol. 121, pp. 285-292.

[44] B. Zhang and A. Bagchi: Finite Element Simulations of Chip Formation and Comparison with Machining Experiment, J. Eng. Ind., 1995, vol. 116, pp. 289-297.

[45] C. Shet and X. Deng, Finite Element Analysis of the Orthogonal Metal Cutting Process, J. Mat. Proc. Tech., 2000, vol. 105, pp. 95-109. 
[46] T. Obikawa, H. Sasahara, and T. Shirakashi, Application of Computational Machining Method to Discontinuous Chip Formation, J. Manuf. Sci. E. Trans. ASME, 1997, vol. 119, pp. 667-674.

[47] J. M. Huang and J. T. Black, An Evaluation of Chip Separation Criteria for the FEM Simulations of Machining, J. Manuf. Sci. E. Trans. ASME, 1996, vol. 118, pp. 545-554.

[48] E. Ceretti, P. Fallbohmer, W. T. Wu, and T. Altan, Application of $2 D$ FEM to Chip Formation in Orthogonal Cutting, J. Mat. Proc. Tech., 1996, vol. 59, pp. 169-180.

[49] E. Ceretti, M. Lucchi, and T. Altan, FEM Simulation of Orthogonal Cutting: Serrated Chip Formation, J. Mat. Proc. Tech., 1999, vol. 95, pp. 17-26.

[50] T. D. Marusich and M. Ortiz, Modelling and Simulation of High-Speed Machining, Int. J. Numer. Meth. Eng., 1995, vol. 38, pp. 3675-3694.

[51] D. J. Benson, An Efficient, Accurate, Simple ALE Method for Nonlinear Finite Element Programs, Comp. Meth. App. Mech. Eng., 1989, vol. 72 (3), pp. 305-350.

[52] D. J. Benson, Computational Methods in Lagrangian and Eulerian Hydrocodes, Comp. Meth. App. Mech. Eng., 1992, vol. 99 (2-3), pp. 235-394.

[53] M. Souli, A. Ouahsine, and L. Lewin, ALE Formulation for Fluid-Structure Interaction Problems, Comp. Meth. App. Mech. Eng., 2000, vol. 190, pp. 659-675.

[54] G. S. Gadala and J. Wang, ALE Formulation and its Application in Solid Mechanics, Comp. Meth. App. Mech. Eng., 1998, vol. 167, pp. 35-55.

[55] M. Movahhedy, M. S. Gadala, and Y. Altintas, Simulation of the Orthogonal Metal Cutting Process using an Arbitrary Lagrangian-Eulerian Finite-Element Method, J. Mat. Proc. Tech., 2000, vol. 103, pp. 267-275.

[56] M. Elmadagli and A. T. Alpas, Metallographic Analysis of the Deformation Microstructure of Copper Subjected to Orthogonal Cutting, Mater. Sci. and Eng. A, 2003, vol. 355, pp. 249-259.

[57] ASTM E 112-96, Annual Book of ASTM Standards, Philadelphia, PA, 1998.

[58] ASM Handbook, Volume 2: Metals Handbook, $9^{\text {th }}$ edition, ASM International, Metal Parks, OH, 1979.

[59] Kennametal Inc., Lathe Tooling, Catalog 1010, Latrobe, PA, 2001.

[60] J. H. Dautzenberg and J. H. Zaat, Quantitative Determination of Deformation by Sliding Wear, Wear, 1973, vol. 23, pp. 9-19. 
[61] M. A. Moore and M. Douthwaite, Plastic Deformation Below Worn Surfaces, Metall. Trans. A, 1976, vol. 7A, pp. 1833-1839.

[62] J. Zhang and A. T. Alpas, Delamination wear in ductile materials containing second phase particles, Mater. Sci. Eng. A, 1993, vol. A160, pp. 25-35.

[63] B. Venkataraman and G. Sundararajan, The Sliding Wear Behaviour of Al-SiC Particulate Composites: II. The Characterization of Subsurface Deformation and Correlation with Wear Behaviour, Acta Mater., 1996, vol. 44, pp. 461-473.

[64] H. Zhang and A. T. Alpas, Quantitative evaluation of plastic strain gradients generated during orthogonal cutting of an aluminum alloy, Mater. Sci. and Eng. A, 2002, vol. A332, pp. 249-254.

[65] F. P. Bowden and D. Tabor, The Friction and Lubrication of Solids: Part II, Oxford University Press, London, England, 1964.

[66] E. Voce, The Relationship Between Stress and Strain for Homogenous Deformation, J. Inst. Met., 1948, vol. 74, pp. 537-549.

[67] J. Takadoum, H. Houmid-Bennani, and D. Mairy, The Wear Characteristics of Silicon Nitride, J. Euro. Ceram. Soc., 1998, vol. 18, pp. 553-556.

[68] G.R. Johnson and W.H. Cook, A Constitutive Model and Data for Metals Subjected to Large Strains, High Strain Rates and High Temperatures, Proceedings of the $7^{\text {th }}$ International Symposium in Ballistics, Hague, The Netherlands, 1983, pp. 541-547.

[69] D. Steinberg, Equation of State and Strength Properties of Selected Materials, Lawrence Livermore National Laboratory, Livermore, CA., 1996.

[70] H. Ernst and M.E. Merchant, Surface Friction of Clean Metals: A Basic Factor in the Metal Cutting Process, Proceedings of the special summer conferences on Friction and Surface Finish, The M.I.T. Press, Cambridge, MA, 1940, pp. 76-101. 


\section{APPENDIX A}

\section{Example Problem: Analysis of a Link Element [24]}

For purposes of demonstrating the discretization of equations, the simplest type of element, a link element, is analyzed under axial loading. In Figure A.1 a bar element with constant cross sectional area $A$, is loaded by a force $f(x)$, which is the force per unit length. The horizontal axis is labeled as both $x$ and $u$, since in the analysis the displacement $\mathrm{u}$ is assumed to be function of $x$ or $u(x)$. The sum of forces along the horizontal direction is:

$$
-A \sigma\left(x-\frac{\Delta x}{2}\right)+A \sigma\left(x+\frac{\Delta x}{2}\right)+f(x) \Delta x=0 .
$$

Approximating the stresses at the end point yields:

$$
-A\left(\sigma(x)-\frac{\Delta x}{2} \frac{d \sigma}{d x}\right)+A\left(\sigma(x)+\frac{\Delta x}{2} \frac{d \sigma}{d x}\right)+f(x) \Delta x=0 .
$$

Simplifying Equation (A-2) and diving through by $\Delta x$ gives the equilibrium equation:

$$
A \frac{d \sigma(x)}{d x}+f(x)=0 \text {. }
$$

Recalling that at infinitesimal strains, the material obeys Hooke's law we can assume:

$$
\sigma(x)=E \frac{d u(x)}{d x}
$$

where $\mathrm{E}$ is the Young's modulus constant through the bar, and $\frac{d u(x)}{d x}$ is the strain resulting from displacement $d u$ in the bar. Substituting Equation (A-4) into Equation (A-3) we obtain the governing differential equation, which is valid within a range of $x_{2} \geq x \geq x_{1}$

$$
A E \frac{d^{2} u}{d x^{2}}+f(x)=0
$$

Equation (A-6) expands equation (A-5) to account for area and Young's modulus as a function of the distance $x$.

$$
\frac{d}{d x}\left(A E \frac{d u}{d x}\right)+f(x)=0
$$


In order to apply the principle of virtual work, a virtual displacement of $\delta u(x)$ is assumed. Multiplying Equation (A-6) by the virtual displacement $\delta u(x)$ and integrating over the length of the bar yields:

$$
\int_{x_{1}}^{x_{2}} \frac{d}{d x}\left(A E \frac{d u}{d x}\right) \delta u(x) d x+\int_{x_{1}}^{x_{2}} f(x) \delta u(x) d x=0 .
$$

The first term must be integrated by parts:

$$
\left[A E \frac{d u}{d x} \delta u(x)\right]_{x=x_{1}}^{x=x_{2}}-\int_{x_{1}}^{x_{2}} A E \frac{d u}{d x} \frac{d \delta u}{d x} d x+\int_{x_{1}}^{x_{2}} f(x) \delta u(x) d x=0 .
$$

Substituting the upper and lower limits in the first term yields

$$
\left.A E \frac{d u}{d x}\right|_{x_{2}} \delta u\left(x_{2}\right)-\left.A E \frac{d u}{d x}\right|_{x_{1}} \delta u\left(x_{1}\right)-\int_{x_{1}}^{x_{2}} A E \frac{d u}{d x} \frac{d \delta u}{d x} d x+\int_{x_{1}}^{x_{2}} f(x) \delta u(x) d x=0
$$

Assuming the force at $x_{1}$ and $x_{2}$ is equal to $P_{1}$ and $P_{2}$, where $P_{1}$ is the in the negative direction, Equation (A-9) can be simplified to:

$$
\int_{x_{1}}^{x_{2}} A E \frac{d u}{d x} \frac{d \delta u}{d x} d x=\int_{x_{1}}^{x_{2}} f(x) \delta u(x) d x+P_{2} \delta u\left(x_{2}\right)+P_{1} \delta u\left(x_{1}\right) .
$$

The displacement $u(x)$ is approximated as a function of the displacement at the left $\left(u_{I}\right)$ and right $\left(u_{2}\right)$ extremities of the bar. The shape functions $N_{1}$ and $N_{2}$ determine the fraction of $u_{1}$ and $u_{2}$ that makes up the displacement $u(x)$ at any point along the bar.

$$
u(x)=u_{1} N_{1}(x)+u_{2} N_{2}(x)
$$

Substituting the approximation in Equation (A-11) into Equation (A-10) yields:

$$
\int_{x_{1}}^{x_{2}} A E\left(u_{1} \frac{d N_{1}}{d x}+u_{2} \frac{d N_{2}}{d x}\right) \frac{d \delta u}{d x} d x=\int_{x_{1}}^{x_{2}} f(x) \delta u(x) d x+P_{2} \delta u\left(x_{2}\right)+P_{1} \delta u\left(x_{1}\right) .
$$

The virtual displacement $\delta u(x)$ can now be assigned a value, and typically it is assigned the value of the shape function $N_{I}(x)$ and $N_{2}(x)$. Substituting in $N_{I}(x)$ and $N_{2}(x)$ results in Equations (A-13) and (A-14), respectively.

$$
\begin{aligned}
& \int_{x_{1}}^{x_{2}} A E\left(u_{1} \frac{d N_{1}}{d x}+u_{2} \frac{d N_{2}}{d x}\right) \frac{d N_{1}}{d x} d x=\int_{x_{1}}^{x_{2}} f(x) N_{1}(x) d x+P_{1} \\
& \int_{x_{1}}^{x_{2}} A E\left(u_{1} \frac{d N_{1}}{d x}+u_{2} \frac{d N_{2}}{d x}\right) \frac{d N_{1}}{d x} d x=\int_{x_{1}}^{x_{2}} f(x) N_{1}(x) d x+P_{1}
\end{aligned}
$$


Manipulation of Equation (A-13) and (A-14), leads to the matrix form of the equilibrium equation or Equation (A-15).

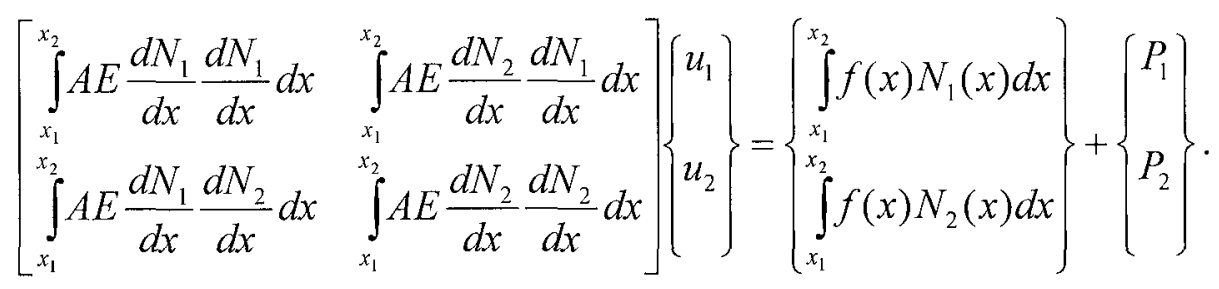

Equation (A-15) is expressed in terms of the global coordinate system and variable $x$ with range $x_{2} \geq x \geq x_{1}$. However it is more practical to write the shape functions in terms of a non-dimensional local coordinate system $s$ with range $1 \geq s \geq-1$, where:

$$
x=\frac{x_{1}+x_{2}}{2}+\frac{L}{2} S
$$

and $\mathrm{L}$ is the length of the element. Derivatives of the shape functions with respect to the local coordinate system are derived by the chain rule, such that:

$$
\frac{d N_{1}}{d x}=\frac{d N_{1}}{d s} \frac{d s}{d x} \text { and } \frac{d N_{2}}{d x}=\frac{d N_{2}}{d s} \frac{d s}{d x}
$$

where $\frac{d s}{d x}$ is called the Jacobian, and can be evaluated by rearranging and taking derivative of equation $(\mathrm{A}-16)$ :

$$
\frac{d s}{d x}=\frac{2}{L}
$$

Substituting Equation (A-17) into Equation (A-15), yields the equilibrium equation in matrix form but also with respect to a local coordinate system $s$.

$$
\begin{aligned}
& {\left[\begin{array}{ll}
\int_{-1}^{1} A E \frac{d N_{1}}{d s} \frac{d N_{1}}{d s}\left[\frac{d s}{d x}\right]^{2} \frac{d x}{d s} d s & \int_{-1}^{1} A E \frac{d N_{2}}{d s} \frac{d N_{1}}{d s}\left[\frac{d s}{d x}\right]^{2} \frac{d x}{d s} d s \\
\int_{-1}^{1} A E \frac{d N_{1}}{d s} \frac{d N_{2}}{d s}\left[\frac{d s}{d x}\right]^{2} \frac{d x}{d s} d s & \int_{-1}^{1} A E \frac{d N_{2}}{d s} \frac{d N_{2}}{d s}\left[\frac{d s}{d x}\right]^{2} \frac{d x}{d s} d s
\end{array}\right]\left\{\begin{array}{l}
u_{1} \\
u_{2}
\end{array}\right\}} \\
& =\left\{\begin{array}{l}
\int_{-1}^{1} f(x(s)) N_{1}(s) \frac{d x}{d s} d s \\
\int_{-1}^{1} f(x(s)) N_{2}(s) \frac{d x}{d s} d s
\end{array}\right\}+\left\{\begin{array}{l}
P_{1} \\
P_{2}
\end{array}\right\}
\end{aligned}
$$




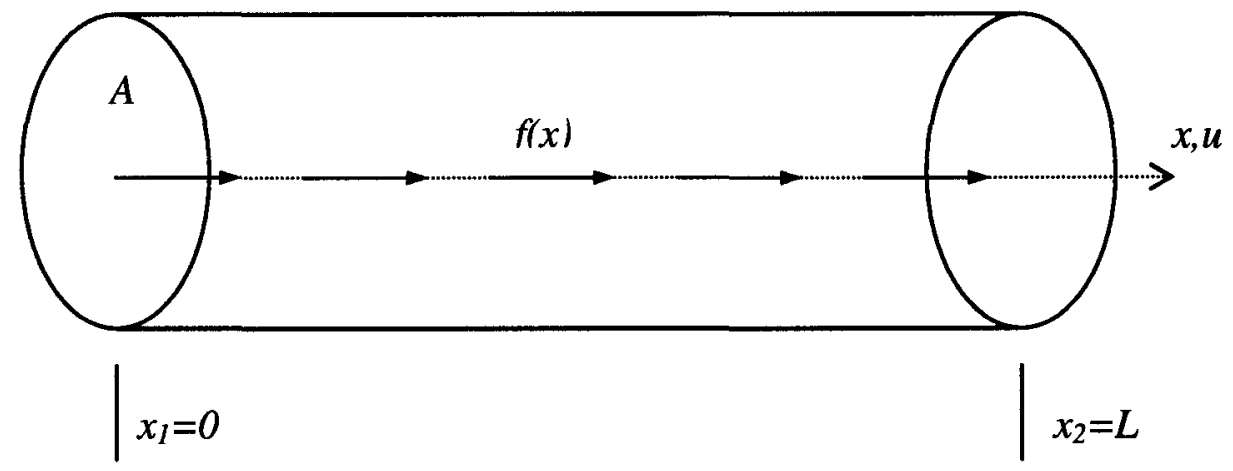

Figure A.1: Axially loaded member.

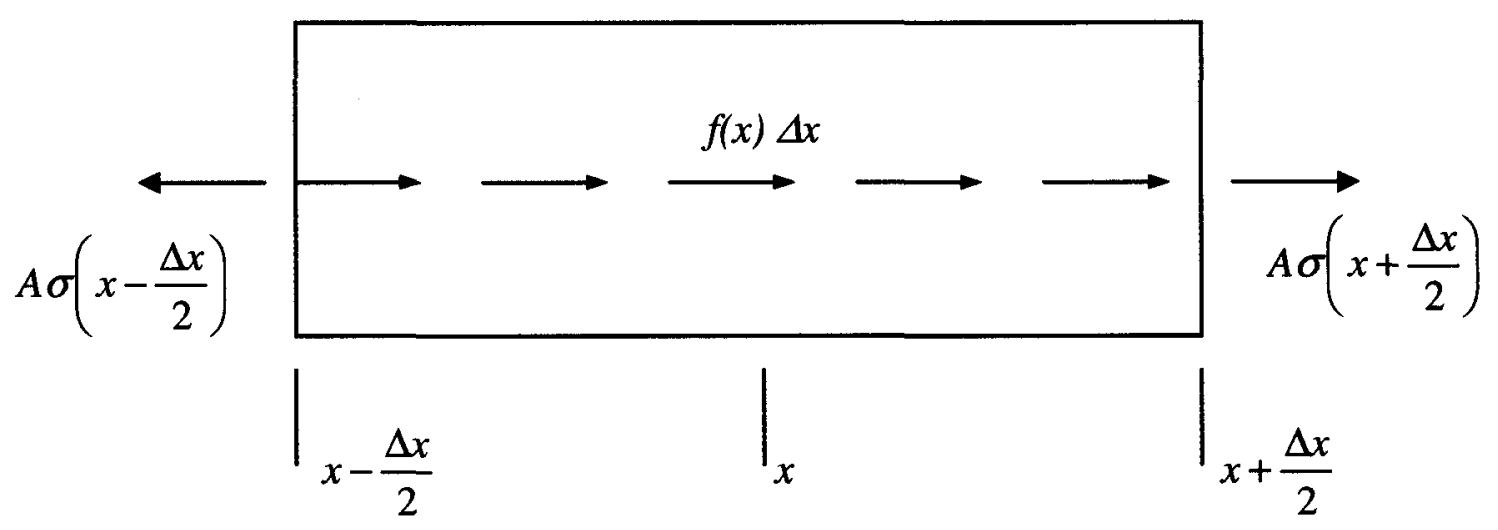

Figure A.2: Free body diagram of the section taken through member in Figure A.1 [24]. 


\section{APPENDIX B \\ Time Integration Techniques in LS-DYNA}

There are two distinct types of solution methods for the equation of motion available in FE analysis; they are the explicit and implicit methods. The time integration algorithm used for the explicit code is the central difference scheme, while the implicit code uses the Newmark's method.

The central difference method [25] can approximate velocity and acceleration as having either constant or linear time dependency during a time step. The linear approximation is used by the central difference scheme in LS-DYNA, and it expresses the acceleration $(\ddot{u})$ and velocity $(\dot{u})$ as:

$$
\begin{gathered}
\dot{u}_{n+\frac{1}{2}}=\frac{1}{2 \Delta t}\left[u_{n+1}-u_{n}\right] \\
\ddot{u}_{n+\frac{1}{2}}=\frac{1}{\Delta t^{2}}\left[u_{n+1}-2 u_{n+\frac{1}{2}}+u_{n}\right]
\end{gathered}
$$

where the subscript notations $n$ represent the time step, such that $n$ and $n+l$ are two consecutive time steps, $n+\frac{1}{2}$ is a mid-point between time steps $n$ and $n+1$, and $\Delta t$ is the duration of the time step interval. According to Equation (A-20) and (A-21), $\dot{u}_{n+\frac{1}{2}}$ and $\ddot{u}_{n+\frac{1}{2}}$ are an average of the velocity and acceleration at the previous time step $(n)$ and the consecutive time step $(n+1)$. When Equations (A-20) and (A-21) are substituted into the equation of motion corresponding to time interval $n+\frac{1}{2}$, the resulting expression takes the form of Equation (A-22).

$$
\left[\frac{[M]}{\Delta t^{2}}+\frac{[C]}{\Delta t}\right] \cdot u_{n+1}=f\left([M][C][K],\left\{u_{n+\frac{1}{2}}\right\},\left\{u_{n}\right\},\left\{F_{n+\frac{1}{2}}\right\}, \Delta t\right)
$$

Equation (A-22) is the governing equation of the central difference scheme. It computes the displacement of nodes at time $n+l$ using only know variables, those either constant through time or those calculated in the previous two time step calculations ( $n$ and $n+\frac{1}{2}$ ). 
Equation (A-22) shows that in order to solve for $u_{n+1}$, the mass matrix and damping matrix must be inverted, or their transpose must be calculated. By lumping the $[M]$ and $[C]$ matrices, and assuming the rigid body accelerations and velocities, respectively, the matrices become diagonal and simple to invert. The condition of stability for the explicit scheme is that the time step can be no larger then the time it takes for an elastic wave to travel through the smallest element, or:

$$
\Delta t_{c}=S \frac{l_{c}}{c}
$$

where $\Delta t_{c}$ is the critical time step, $l_{c}$ is the smallest finite element length, $S$ is the safety factor ( 0.9 by default), and $c$ is the wave propagation velocity defined by Equation (A-24) with $c$ defined as a function of material density $(\rho)$, Young's modulus $(E)$, and Poisson's ratio $(v)$.

$$
\begin{array}{lc}
\text { 1-D truss element } & c=\sqrt{\frac{E}{\rho}} \\
\text { 2-D shell elements } & c=\sqrt{\frac{E}{\rho \cdot\left(1-v^{2}\right)}} \\
\text { 3-D solid elements } \quad c=\sqrt{\frac{E \cdot(1-v)}{\rho \cdot(1+v) \cdot(1-2 v)}}
\end{array}
$$

The definition for length of the smallest finite element $\left(l_{c}\right)$ for truss, shell, and solid elements, can be obtained in the LS-DYNA Theoretical Manual [27].

The Newmark's method [26] approximates the displacement and velocity vector as:

$$
\begin{gathered}
u_{n+1}=u_{n}+\Delta t_{n} \dot{u}_{n}+\Delta t_{n}{ }^{2} \cdot\left[\left(\frac{1}{2}-\beta\right) \ddot{u}_{n}+\beta \cdot \ddot{u}_{n+1}\right] \\
\left.\dot{u}_{n+1}=\dot{u}_{n}+\Delta t_{n} \cdot\left[(1-\gamma) \ddot{u}_{n}+\gamma \cdot \ddot{u}_{n+1}\right)\right]
\end{gathered}
$$

where $\beta$ and $\gamma$ are parameters, which customize the approximations of velocity and displacement as constant, linear, and up to fourth order accurate within a time step. For the implicit scheme the parameters are set to $\beta=^{1 / 4}$ and $F^{1 / 2}$, which approximate constant 
displacement and velocity vectors over a time step $(\Delta t)$ and make the scheme unconditionally stable. Once the parameters are substituted into Equations (A-25) and (A-26) expressions for $\ddot{u}_{n+1}$ and $\dot{u}_{n+1}$ are derived and substituted into the equation of motion corresponding to the time interval of $n+1$. Equation (A-27), shows the form of the expression, which results from these substitutions.

$$
\left[\frac{4}{\Delta t^{2}}[M]+\frac{2}{\Delta t}[C]+[K]\right] \cdot u_{n+1}=f\left([M],[C][K],\left\{u_{n}\right\},\left\{\dot{u}_{n}\right\},\left\{\ddot{u}_{n}\right\}\left\{F_{n+1}\right\}, \Delta t\right)
$$

Equation (A-27) is the governing equation of the implicit scheme. It computes the displacement of nodes at time $n+1$, from nodal properties from the previous time step. Contrary to the central difference scheme, the stiffness matrix $[K]$ must be inverted in addition to the mass and damping matrices. Since the stiffness matrix is not a diagonal, finding the transpose of this matrix can prove to be a time-consuming process. 


\section{APPENDIX C \\ Sample LS-DYNA input for Lagrangian Element Formulation Model}

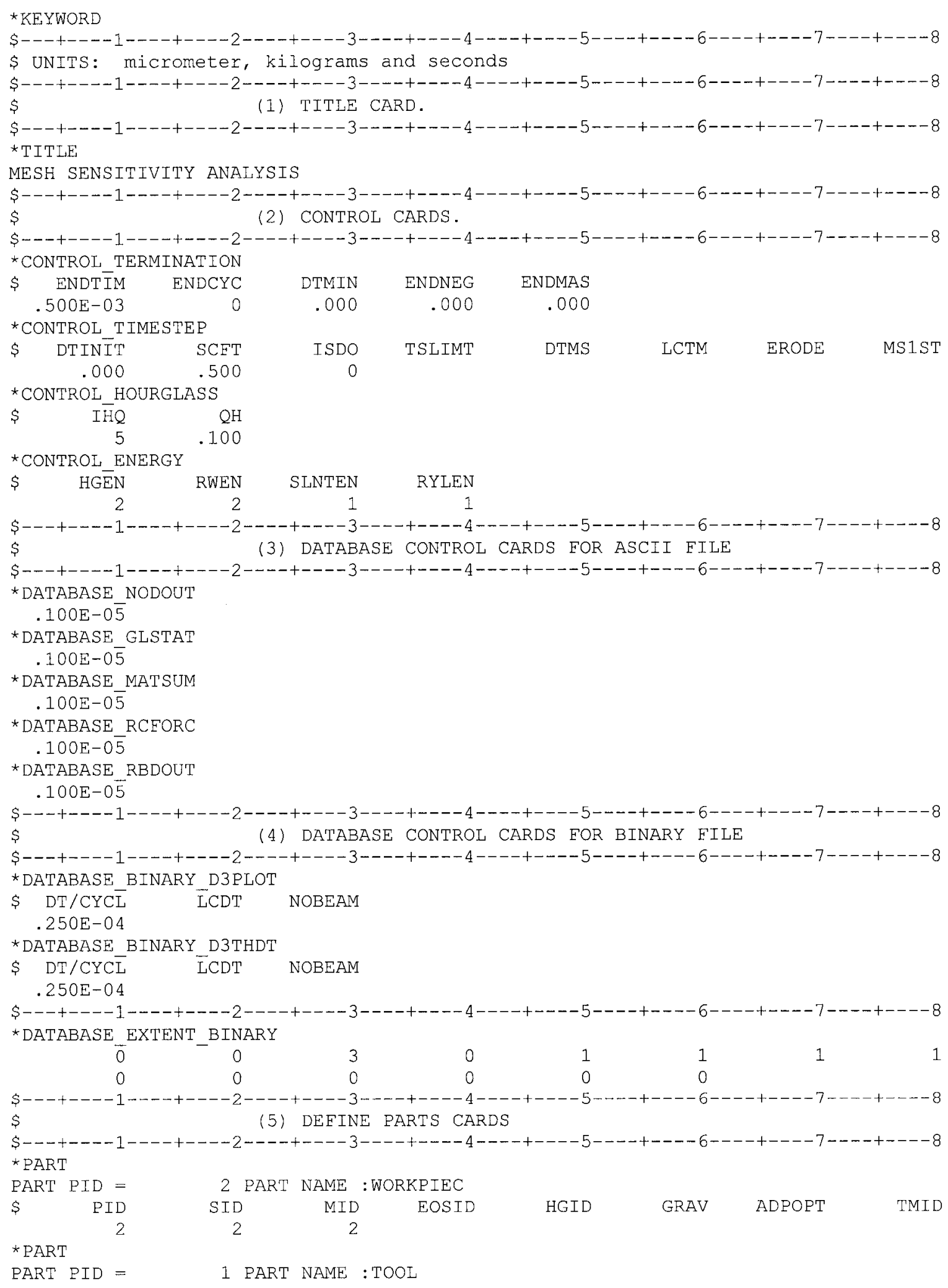




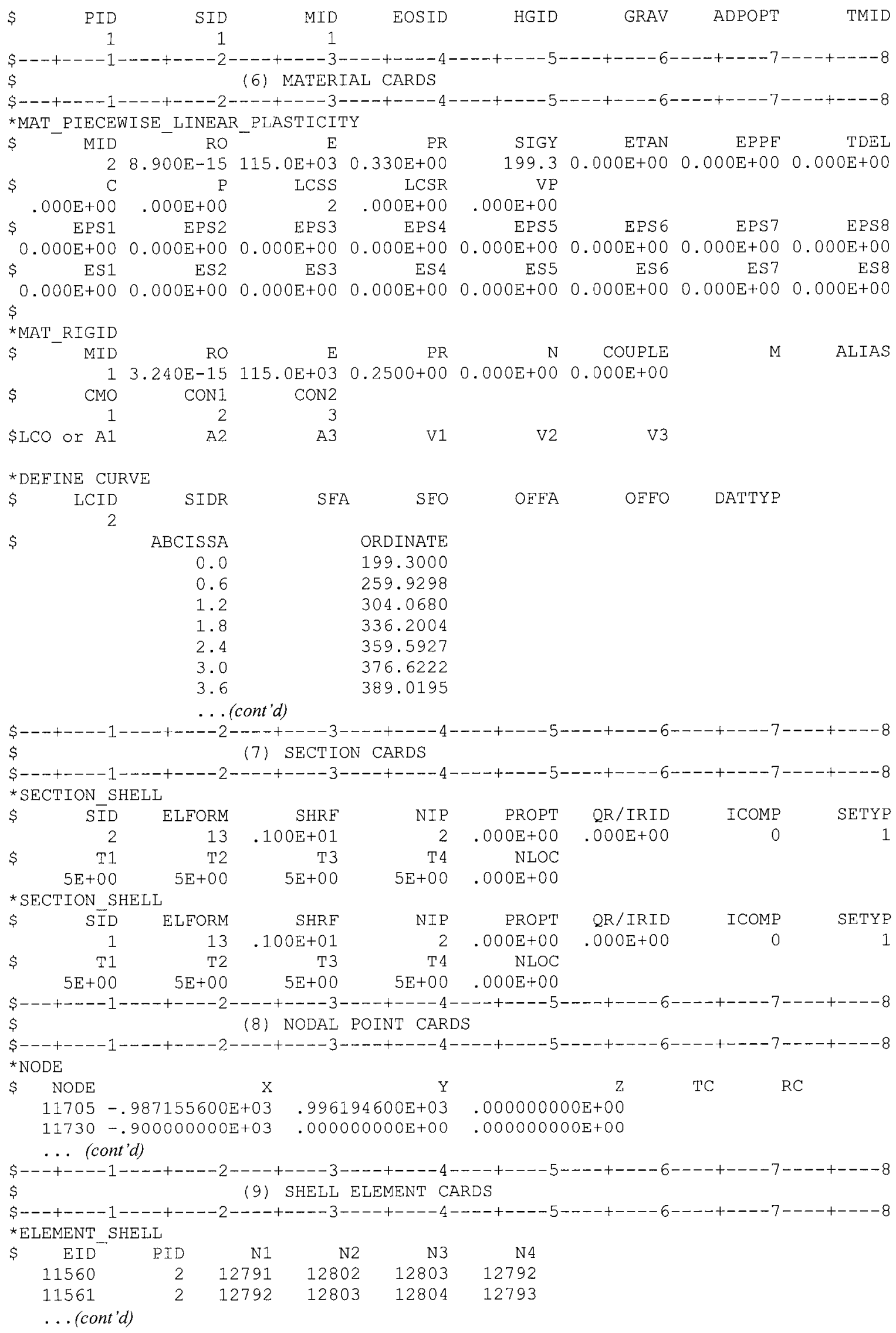




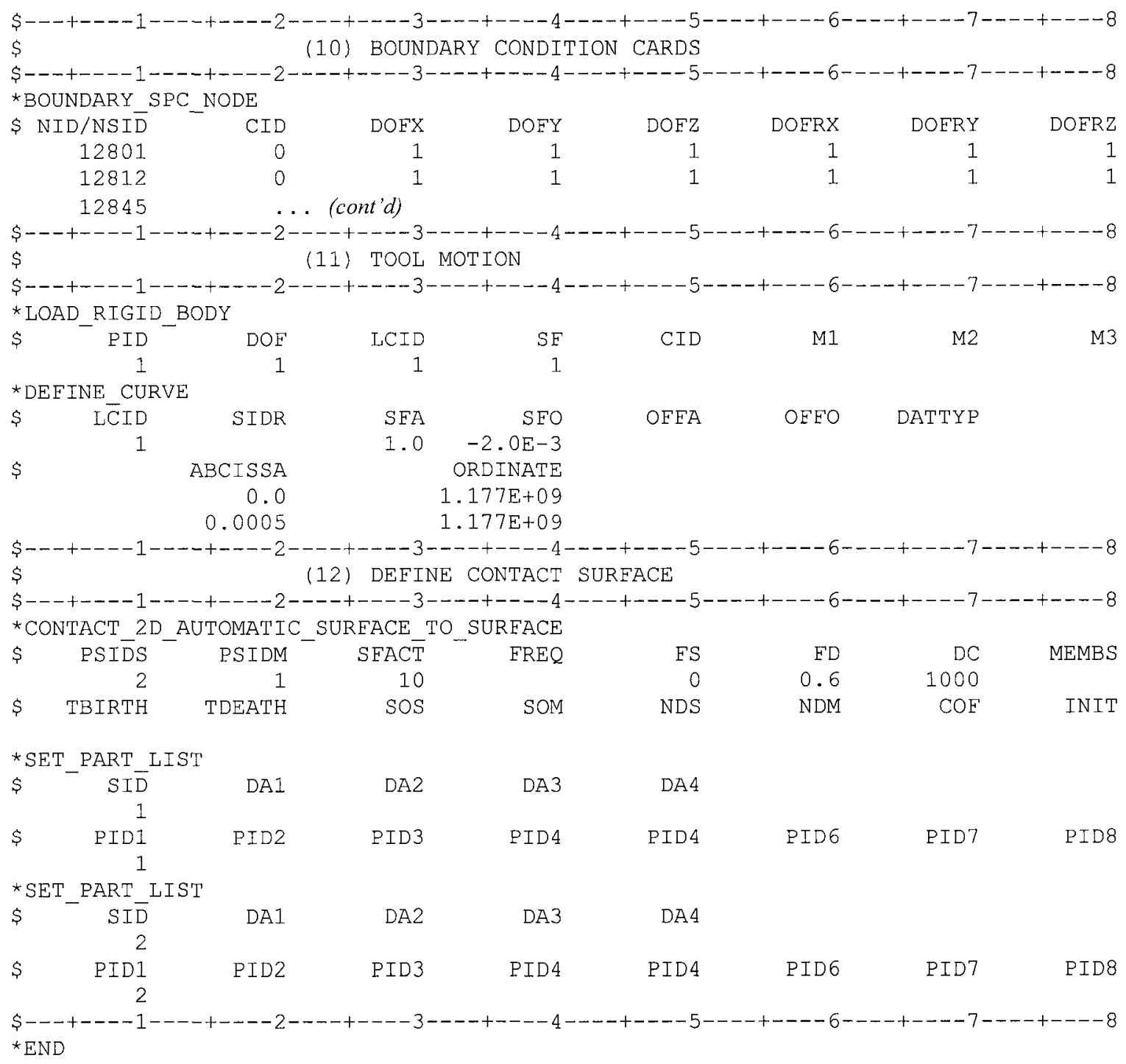


APPENDIX D

Sample LS-DYNA input for Lagrangian Element Formulation Model with Full Adaptivity

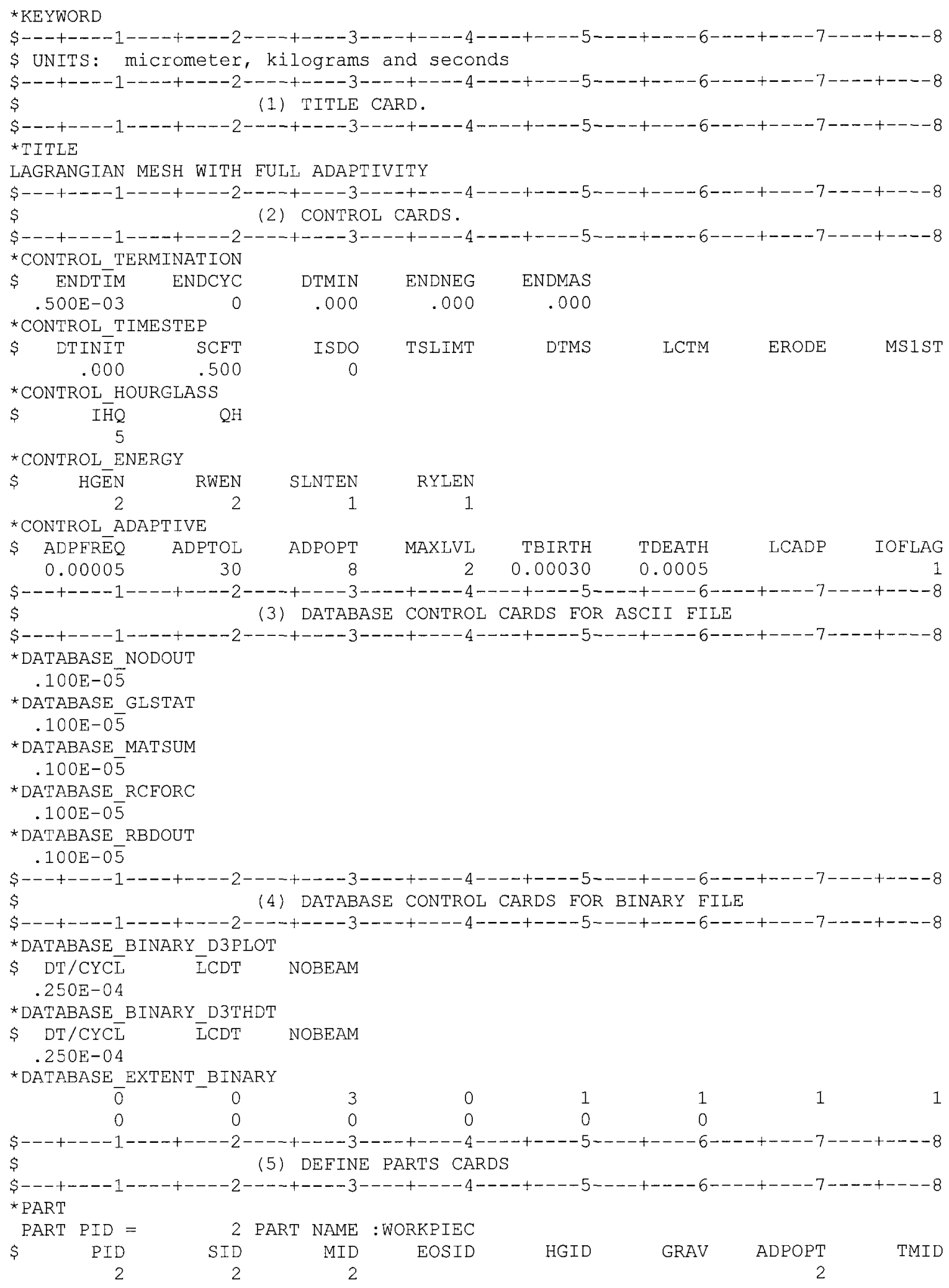




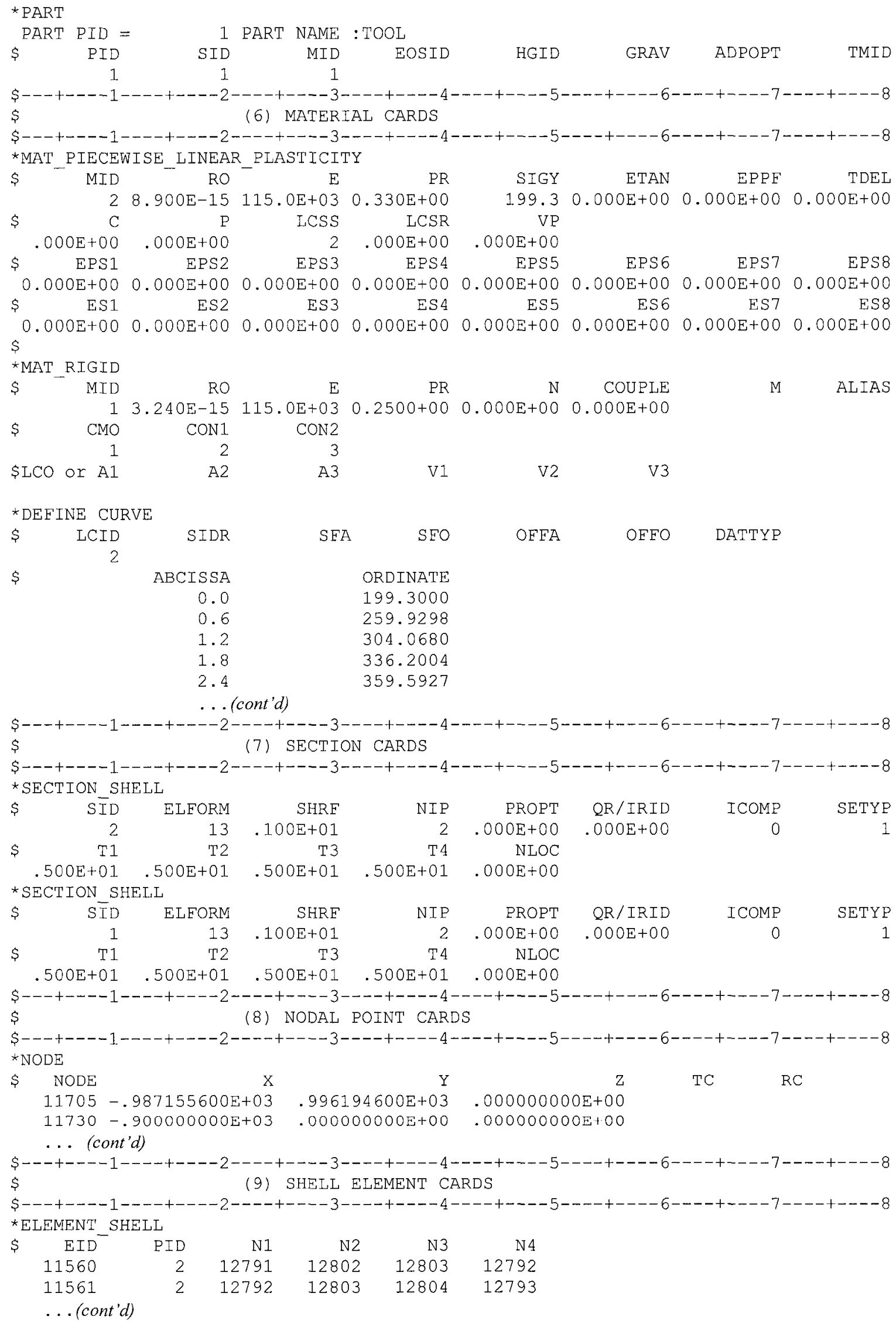




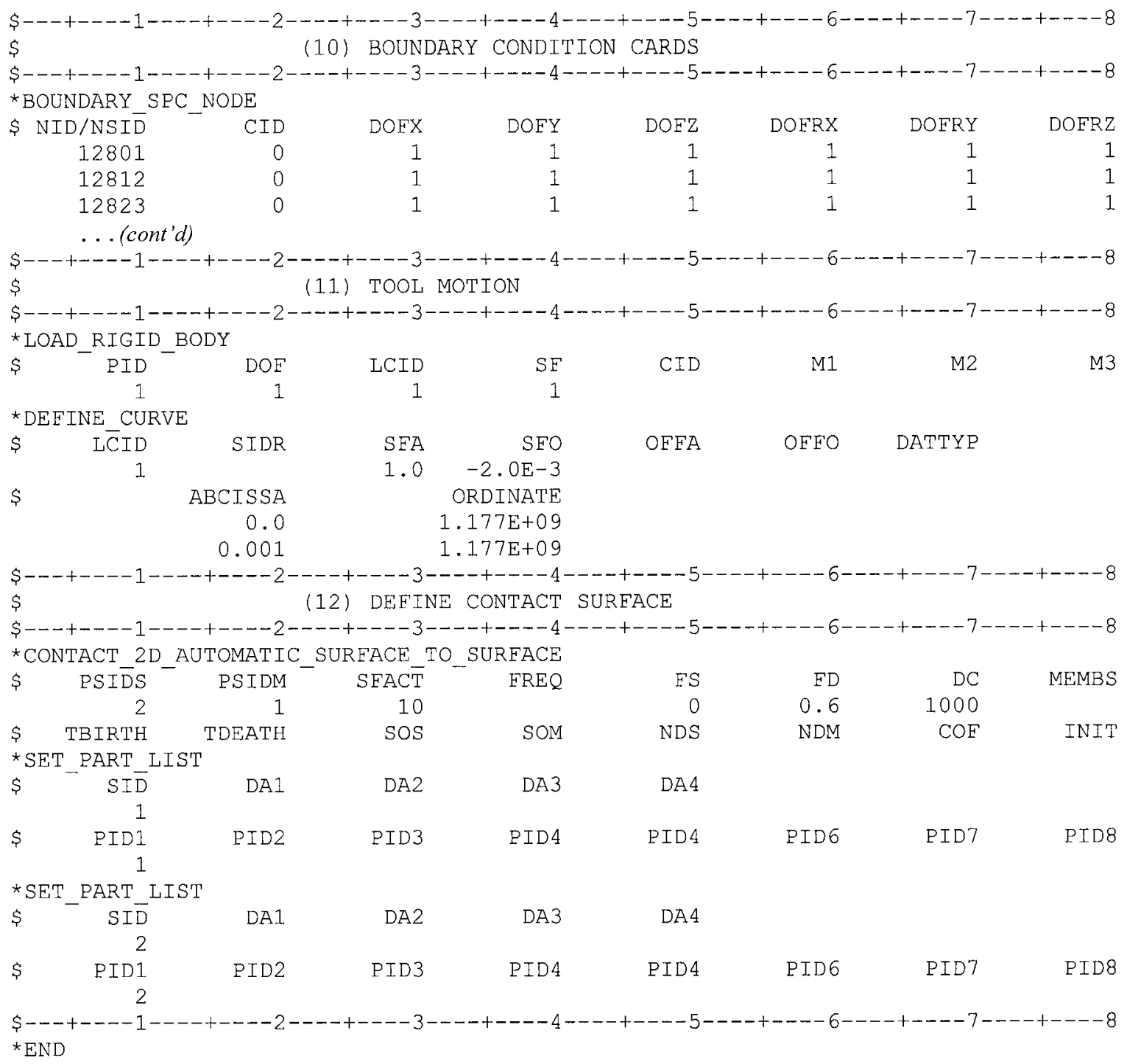




\section{APPENDIX E \\ Sample LS-DYNA input for Lagrangian Element Formulation Model with Partial Adaptivity}

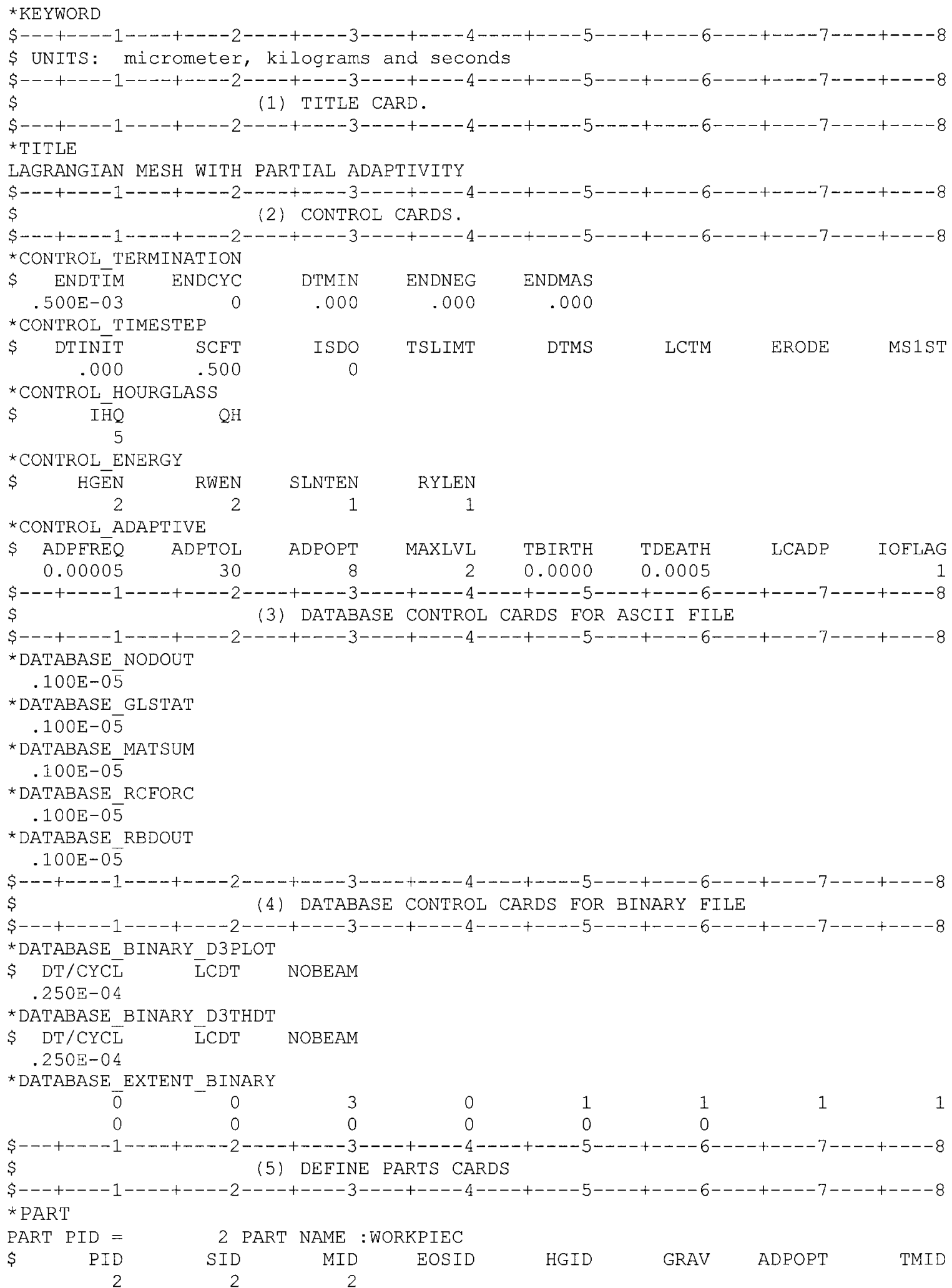




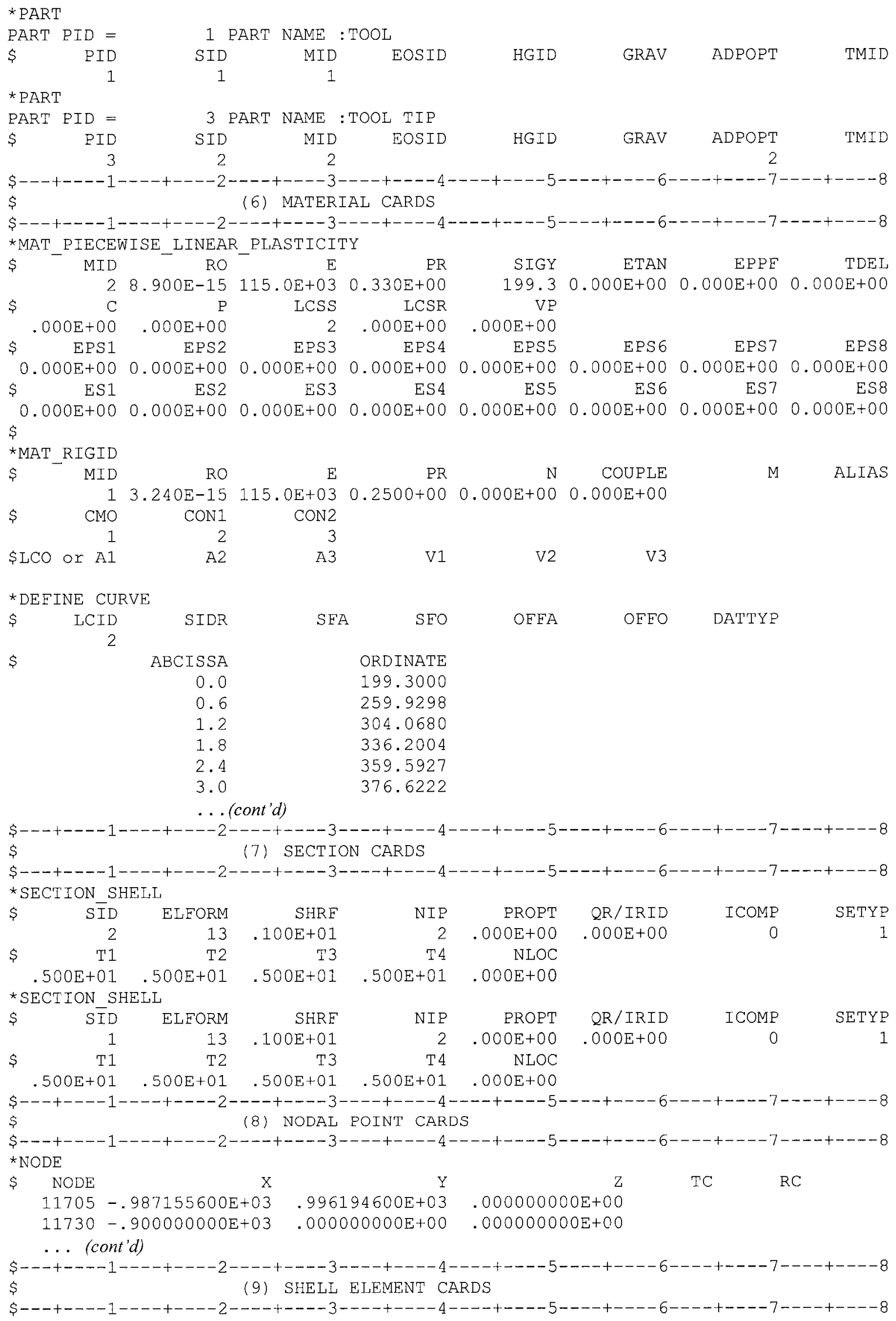




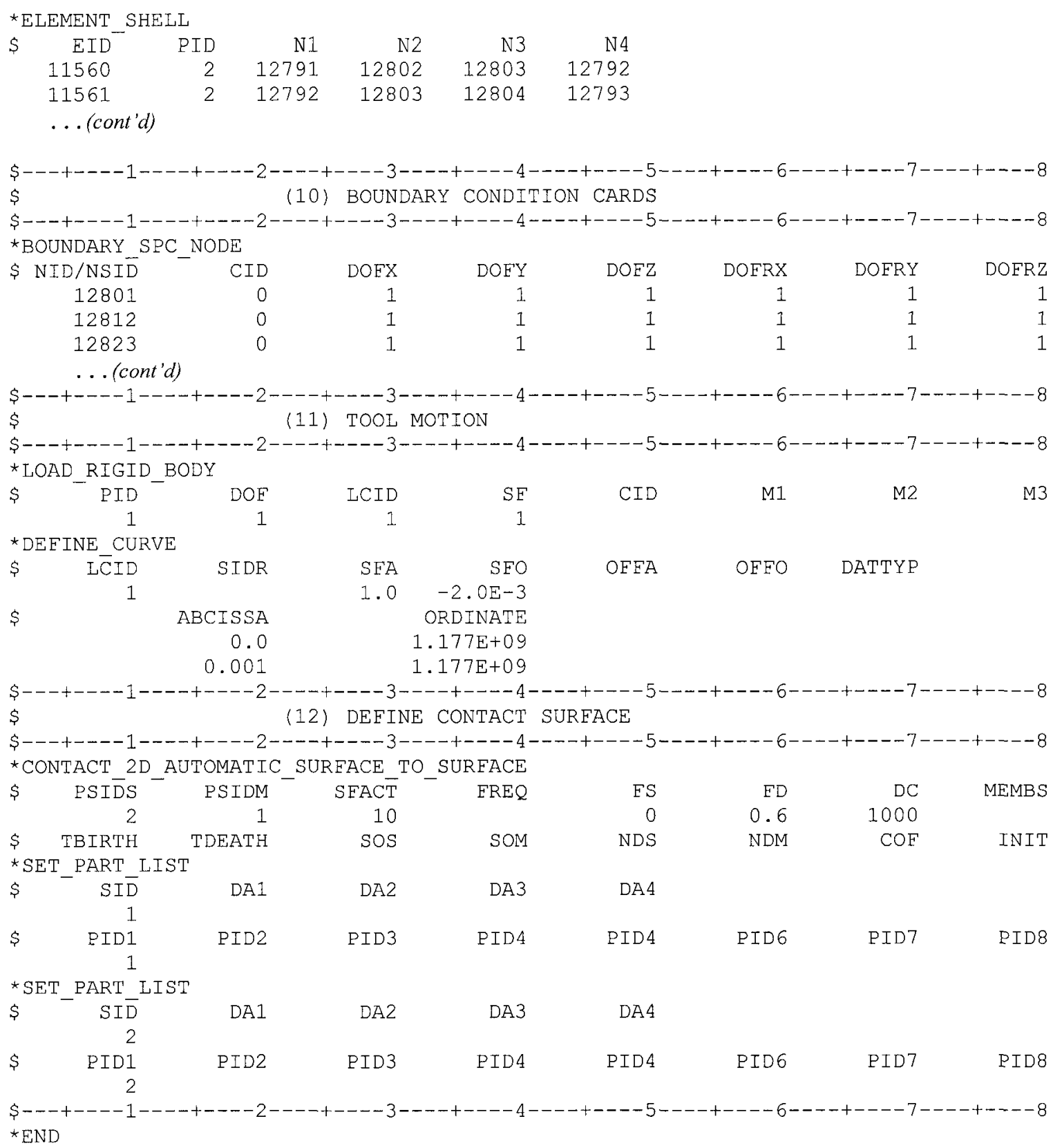




\section{APPENDIX F \\ Sample LS-DYNA input for ALE Element Formulation Model for Steady State Chip Formation}

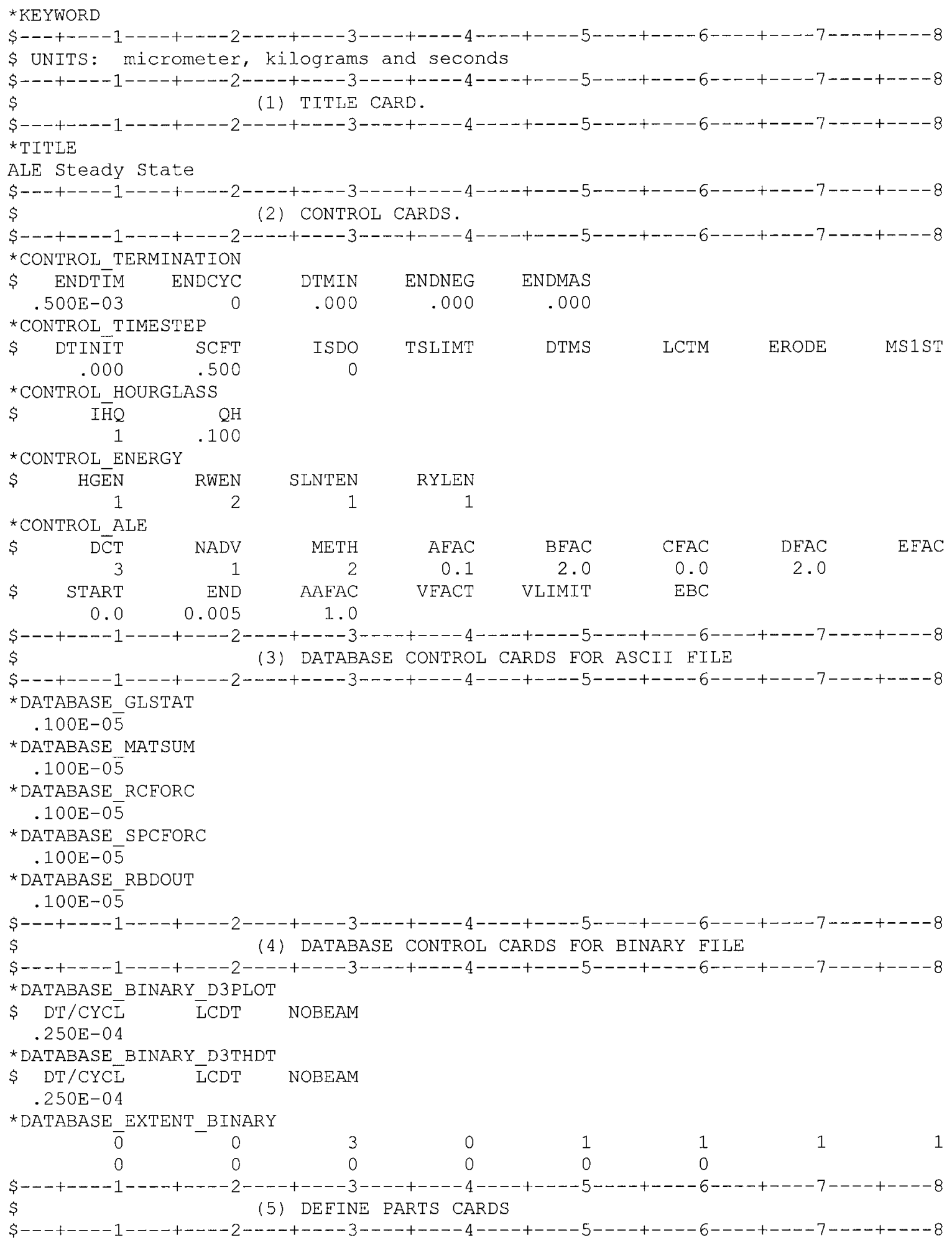




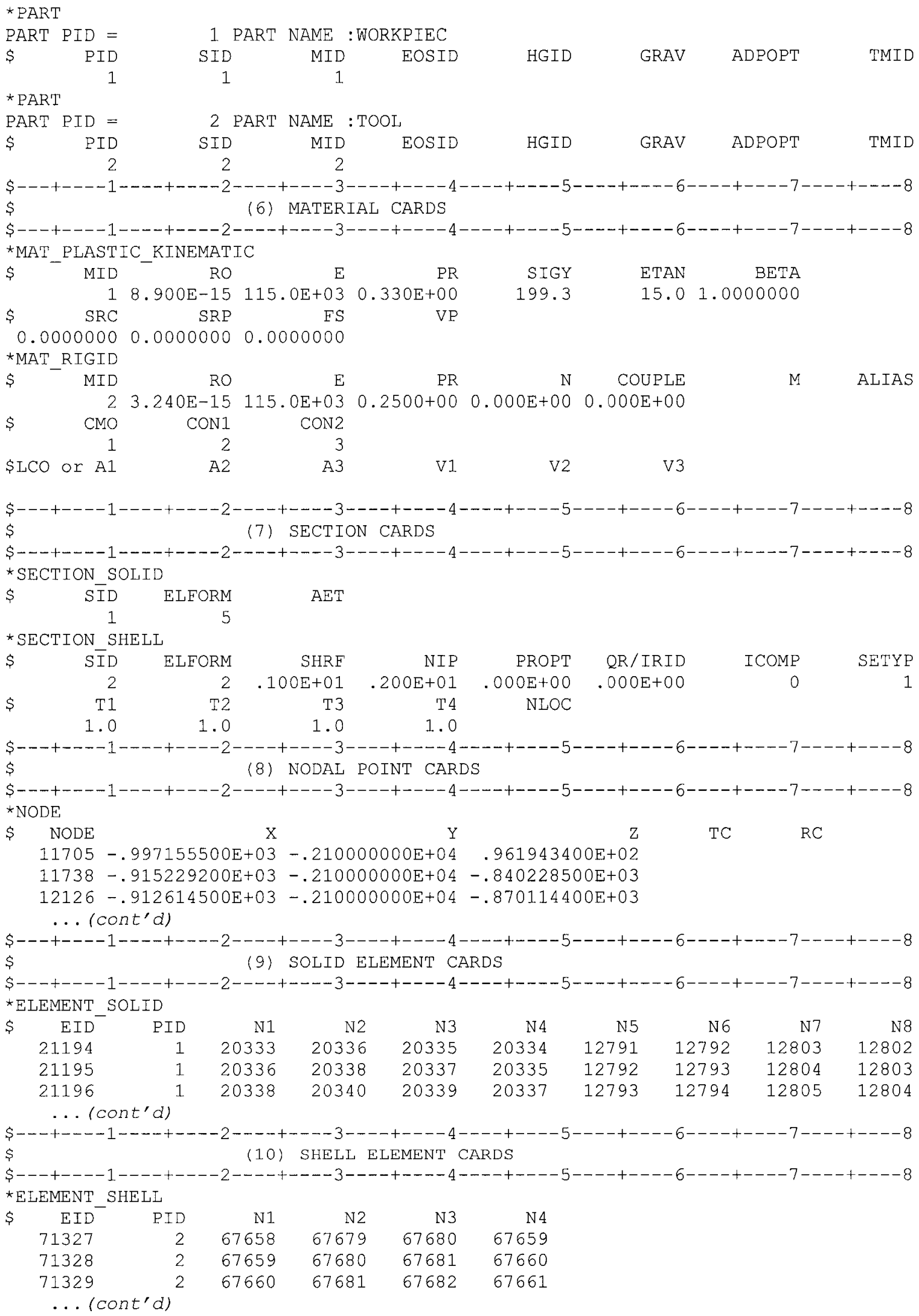




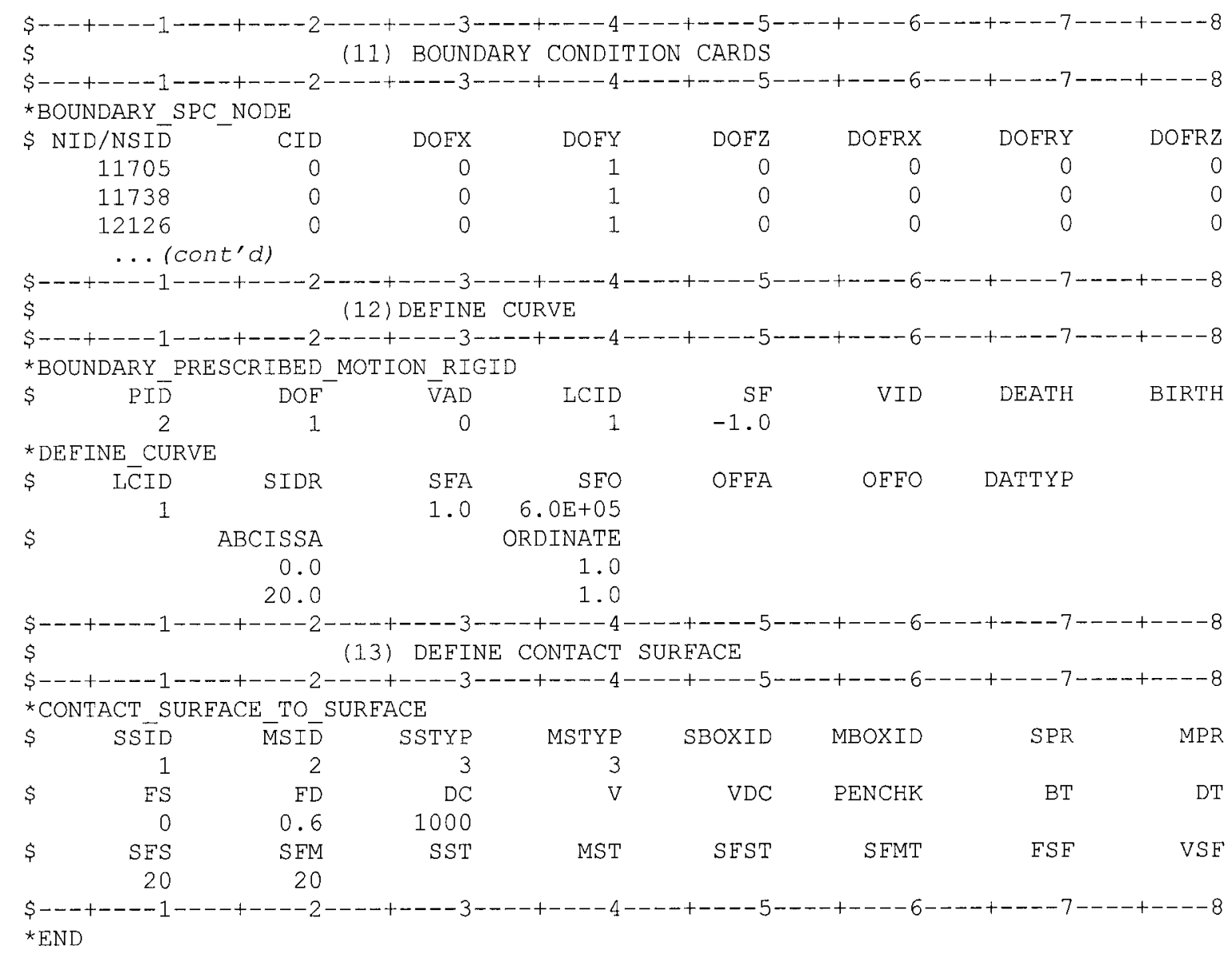




\section{APPENDIX G \\ Sample LS-DYNA input for ALE Element Formulation Model for Incipient Chip Formation}

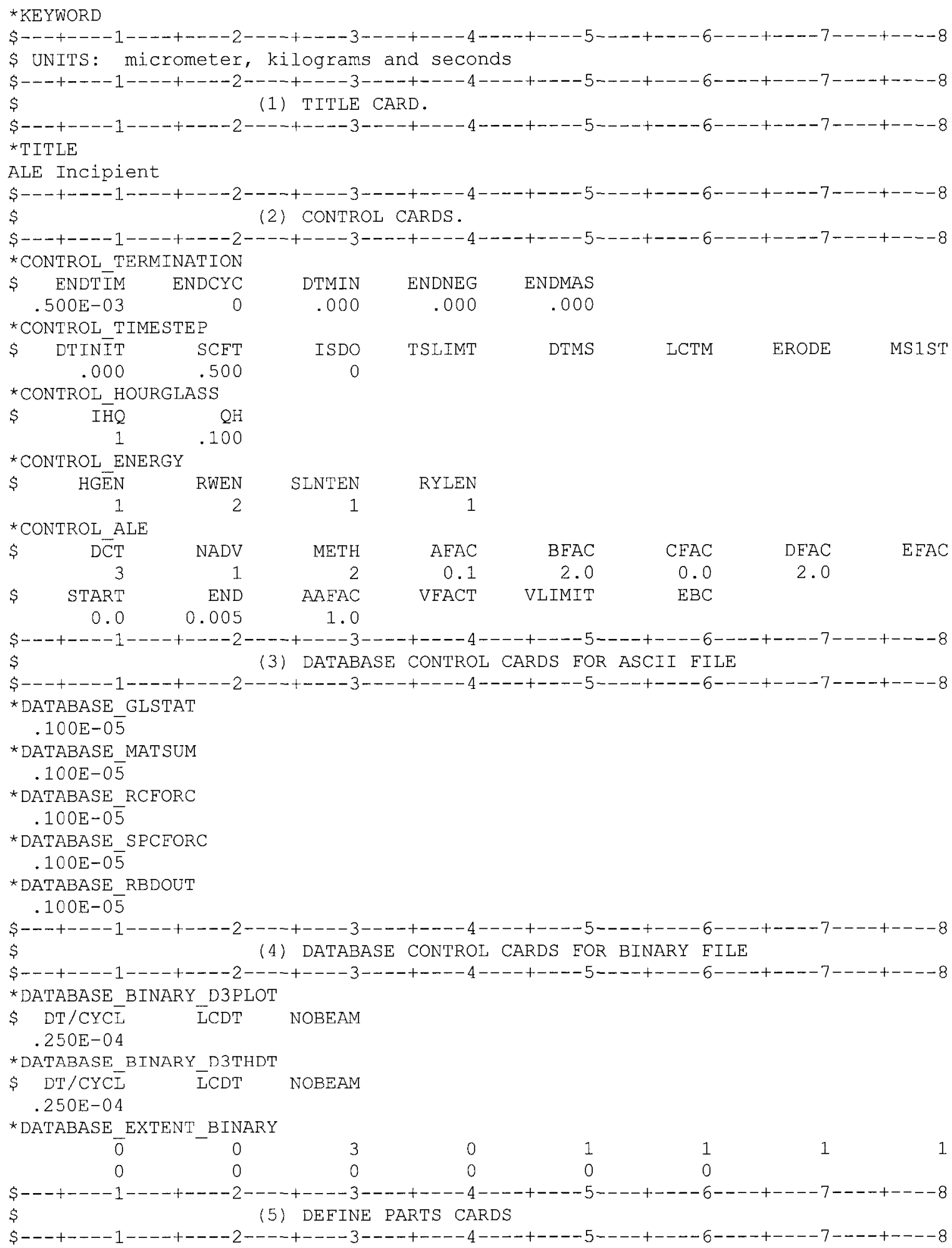




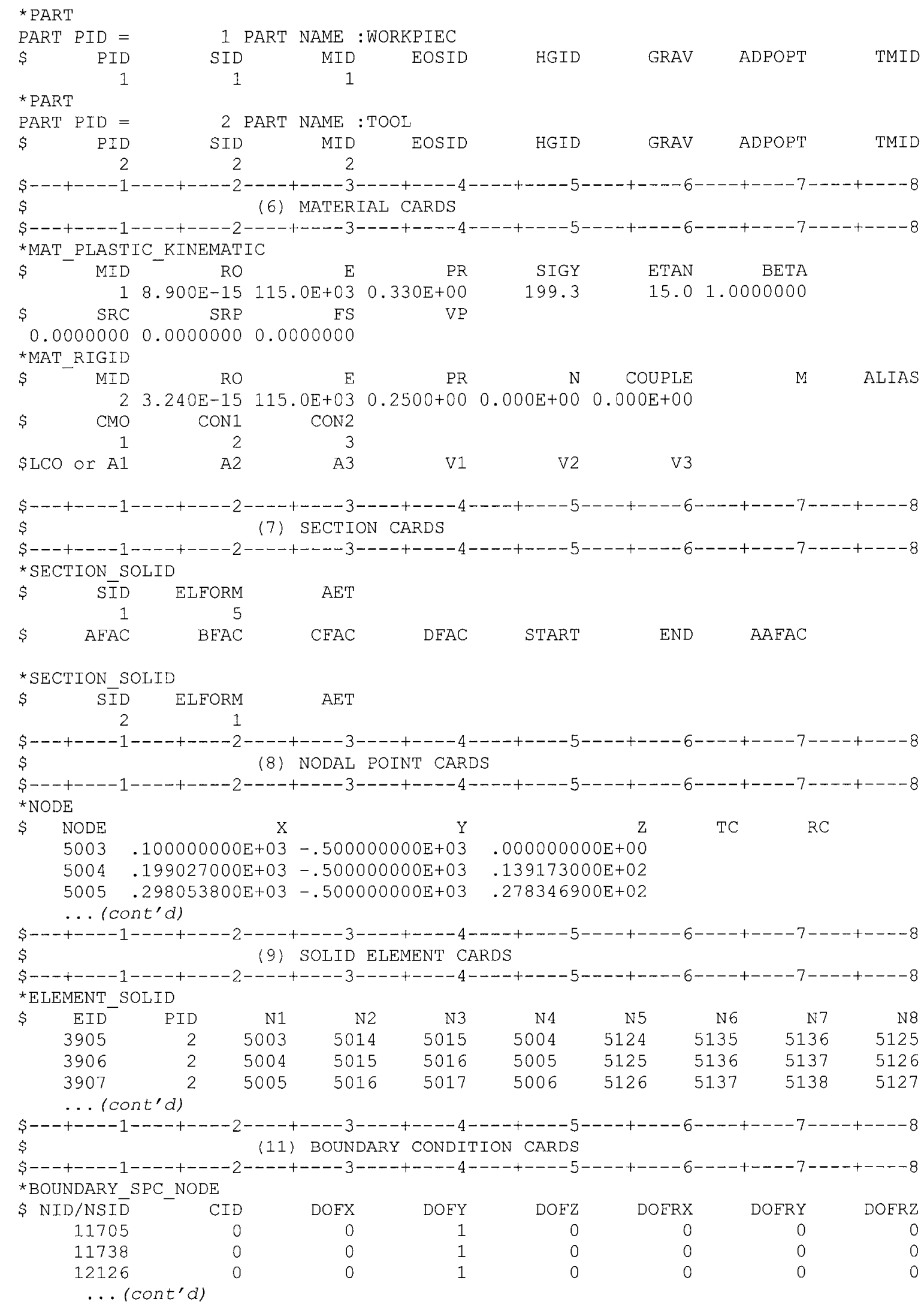




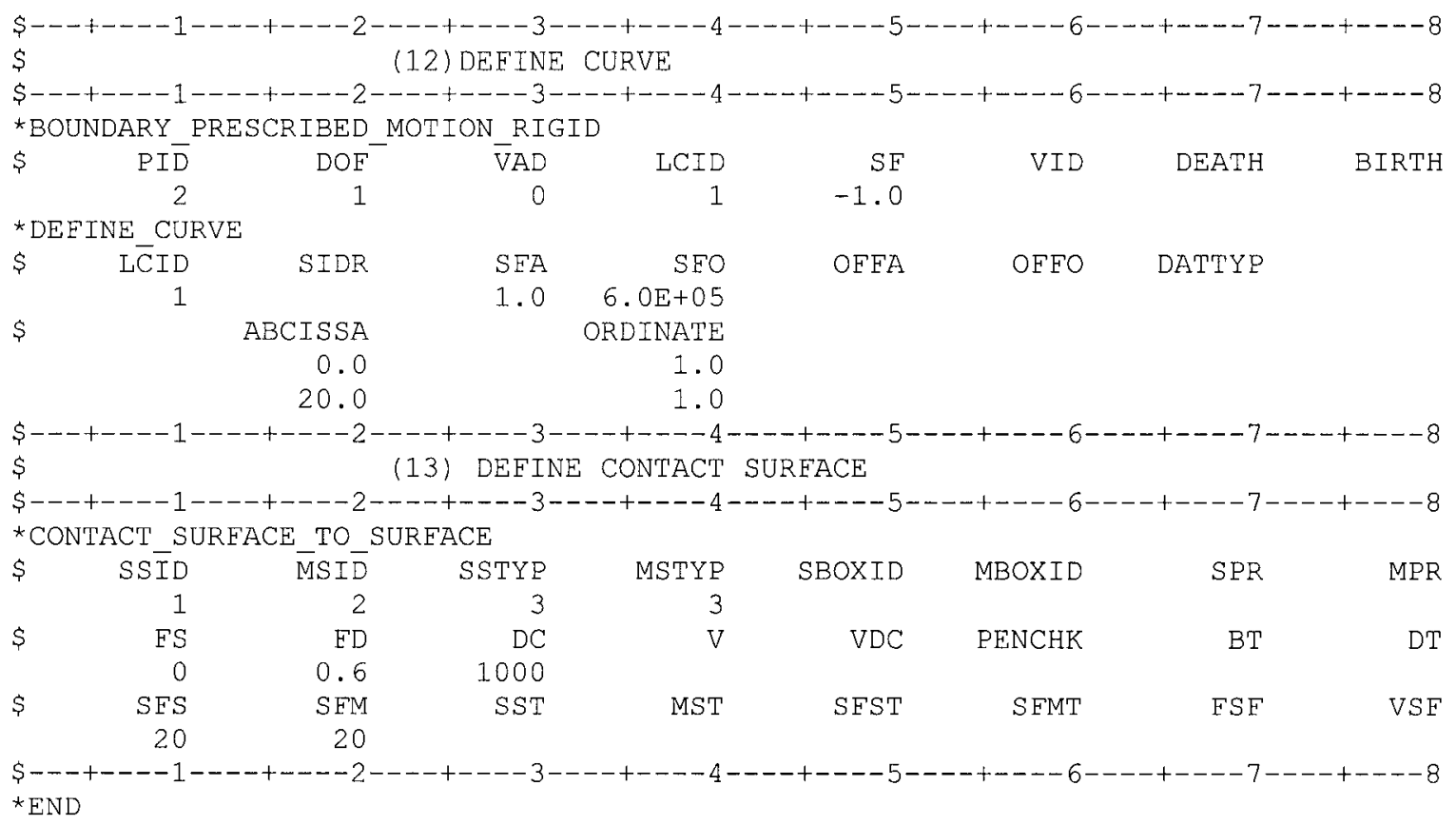




\section{APPENDIX H}

\section{Sample LS-DYNA input for Eulerian Element Formulation Study with Material Model 1}

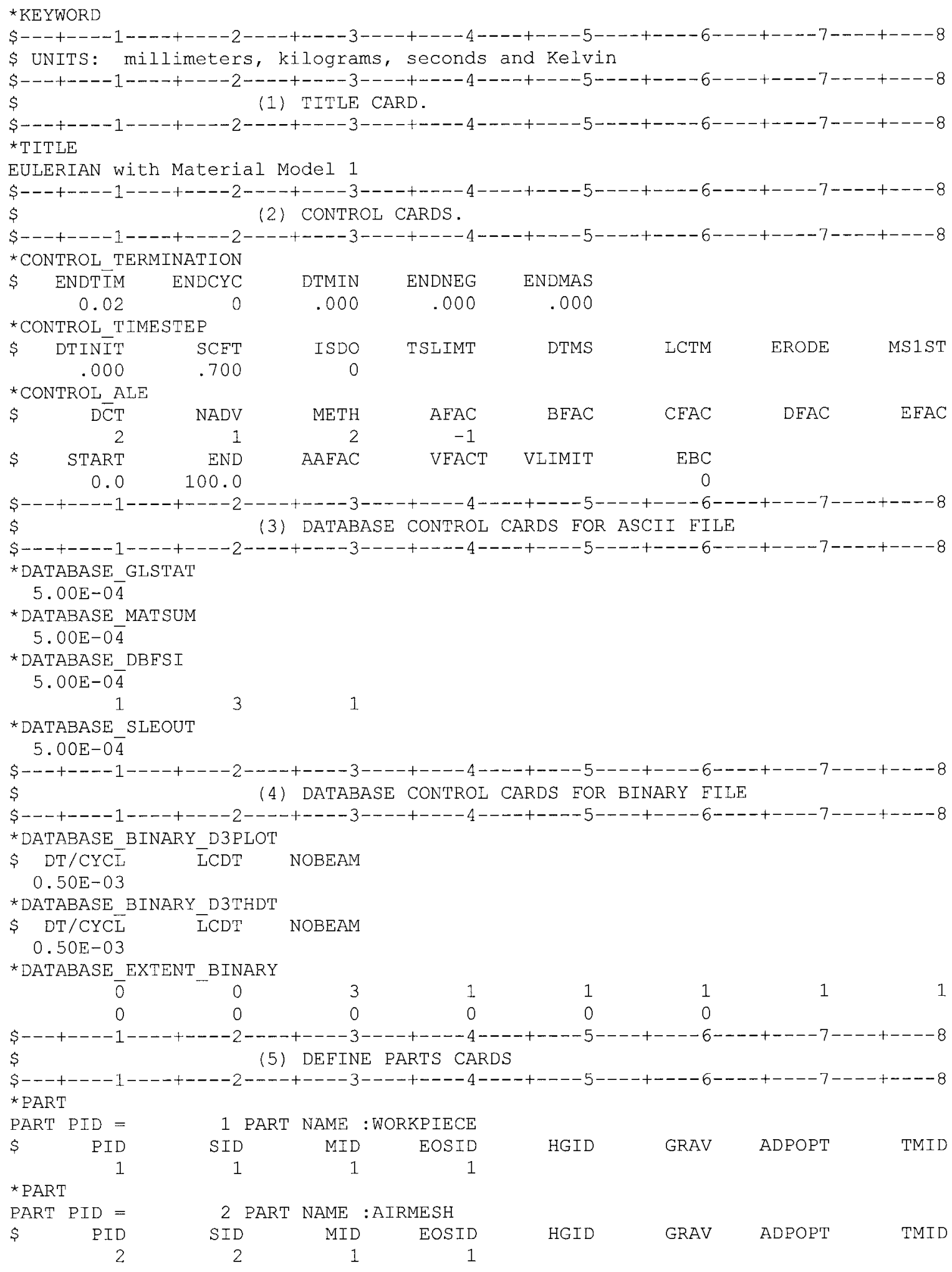




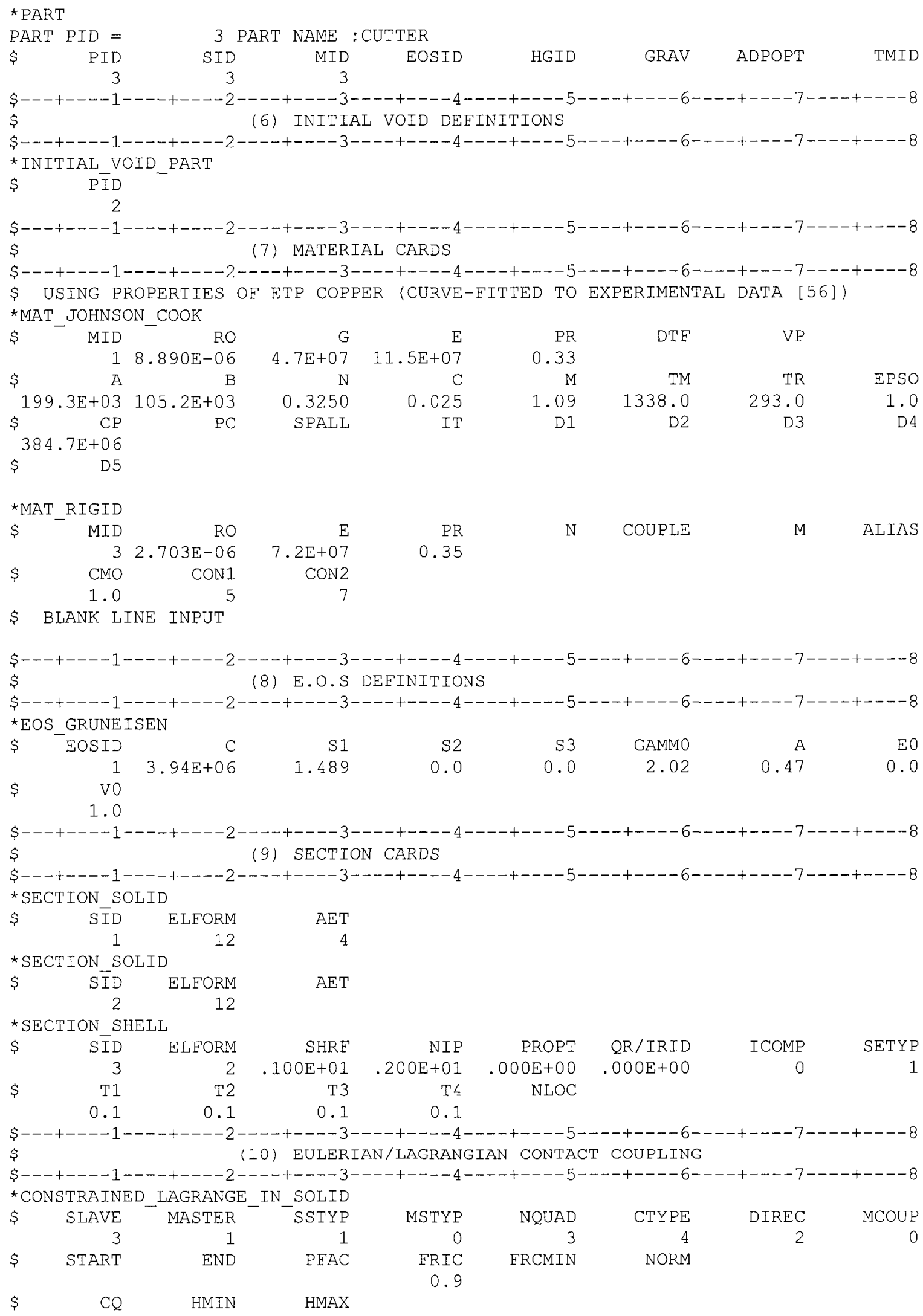




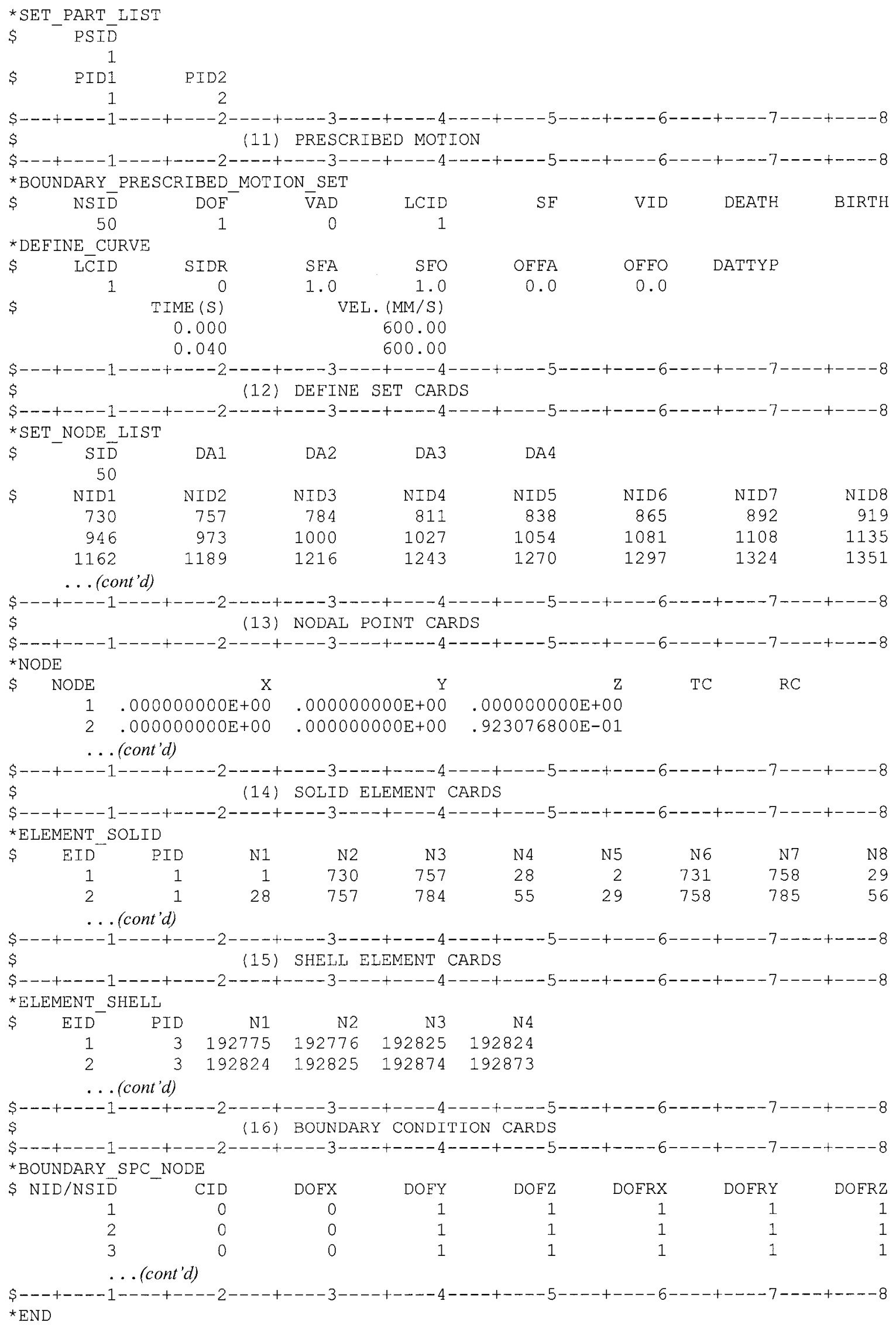




\section{APPENDIX I}

\section{Sample LS-DYNA input for Eulerian Element Formulation Study with Material}

\section{Model 2}

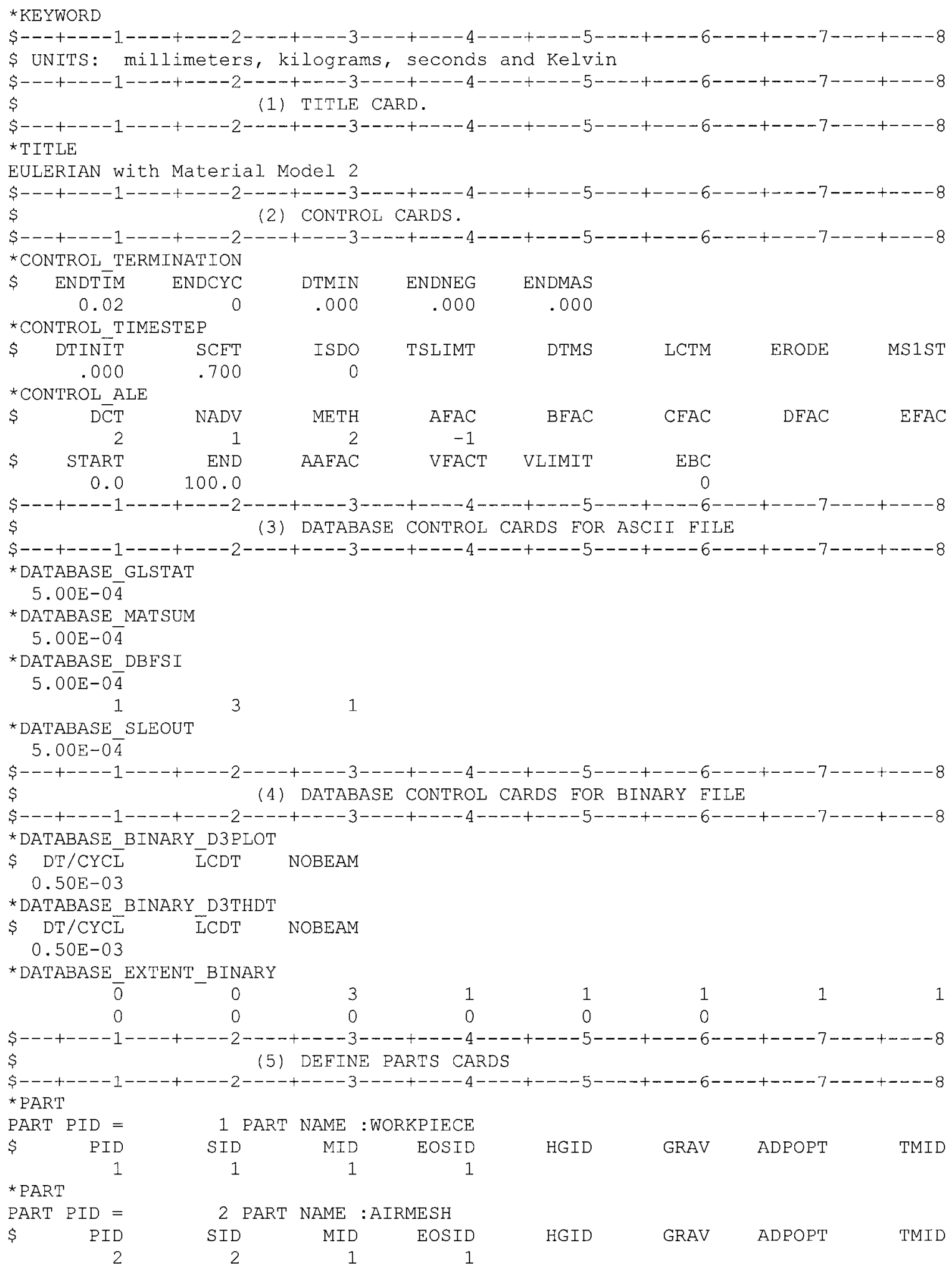




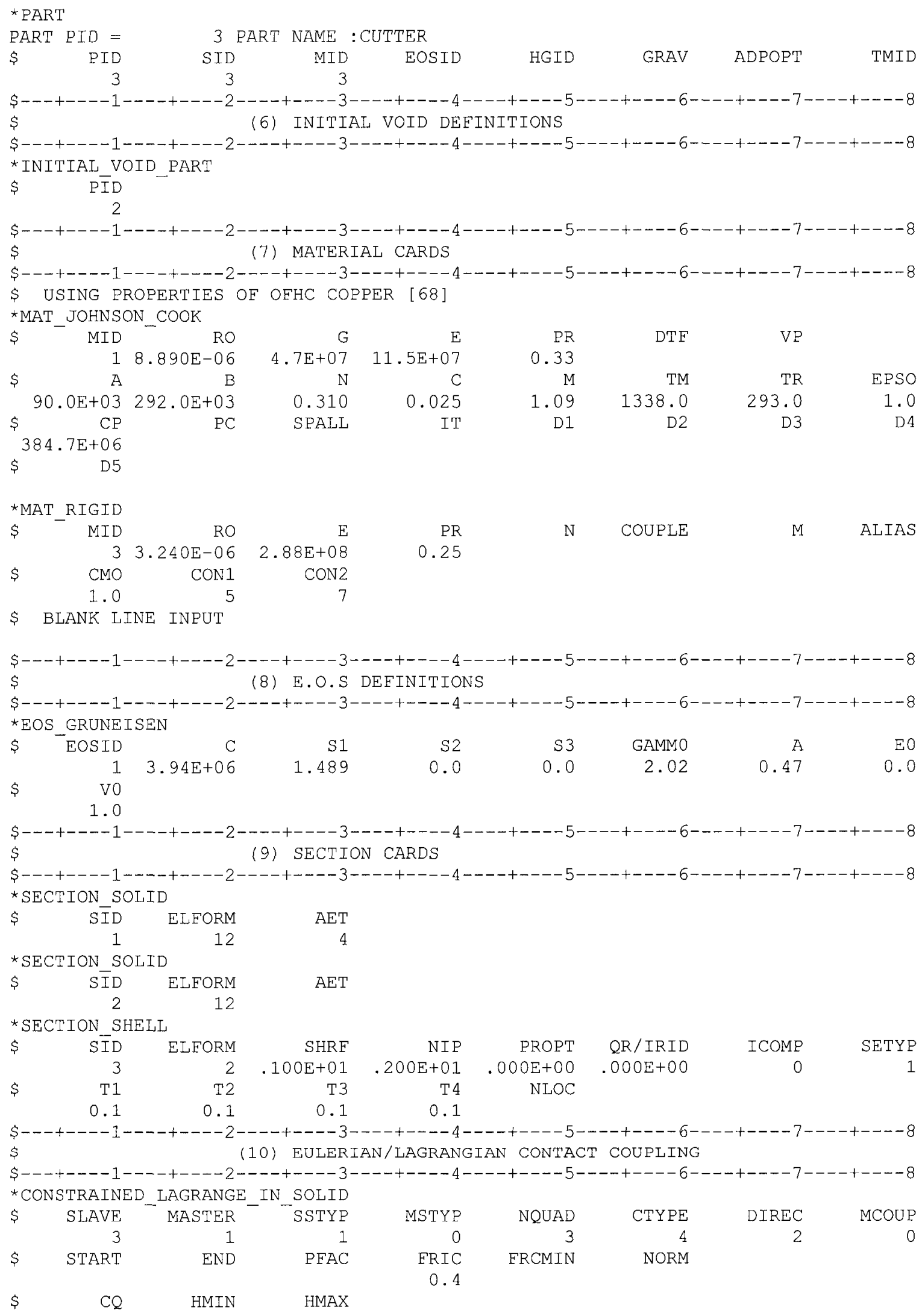




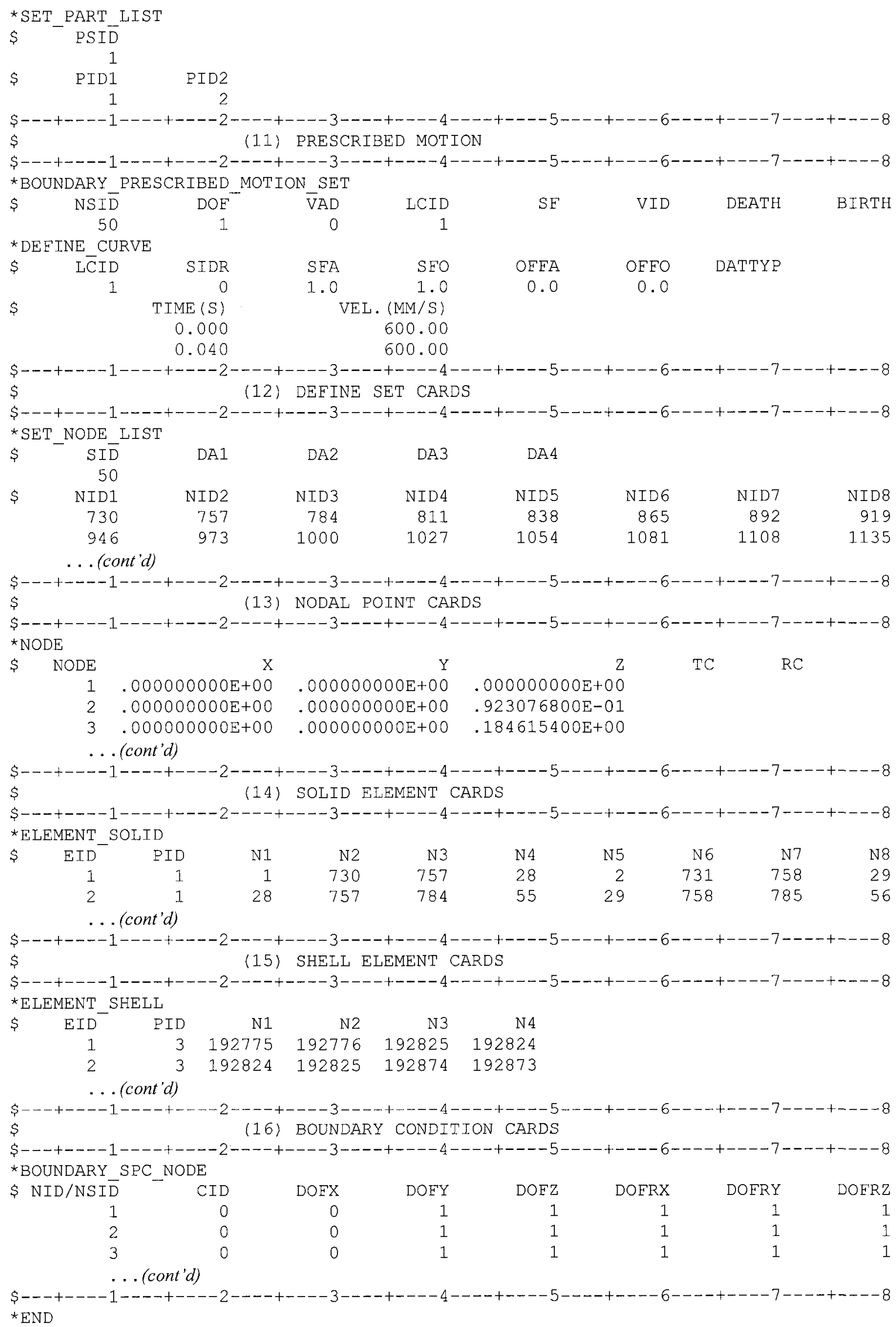




\section{APPENDIX J \\ Sample LS-DYNA input for Eulerian Element Formulation Study with Material Model 3}

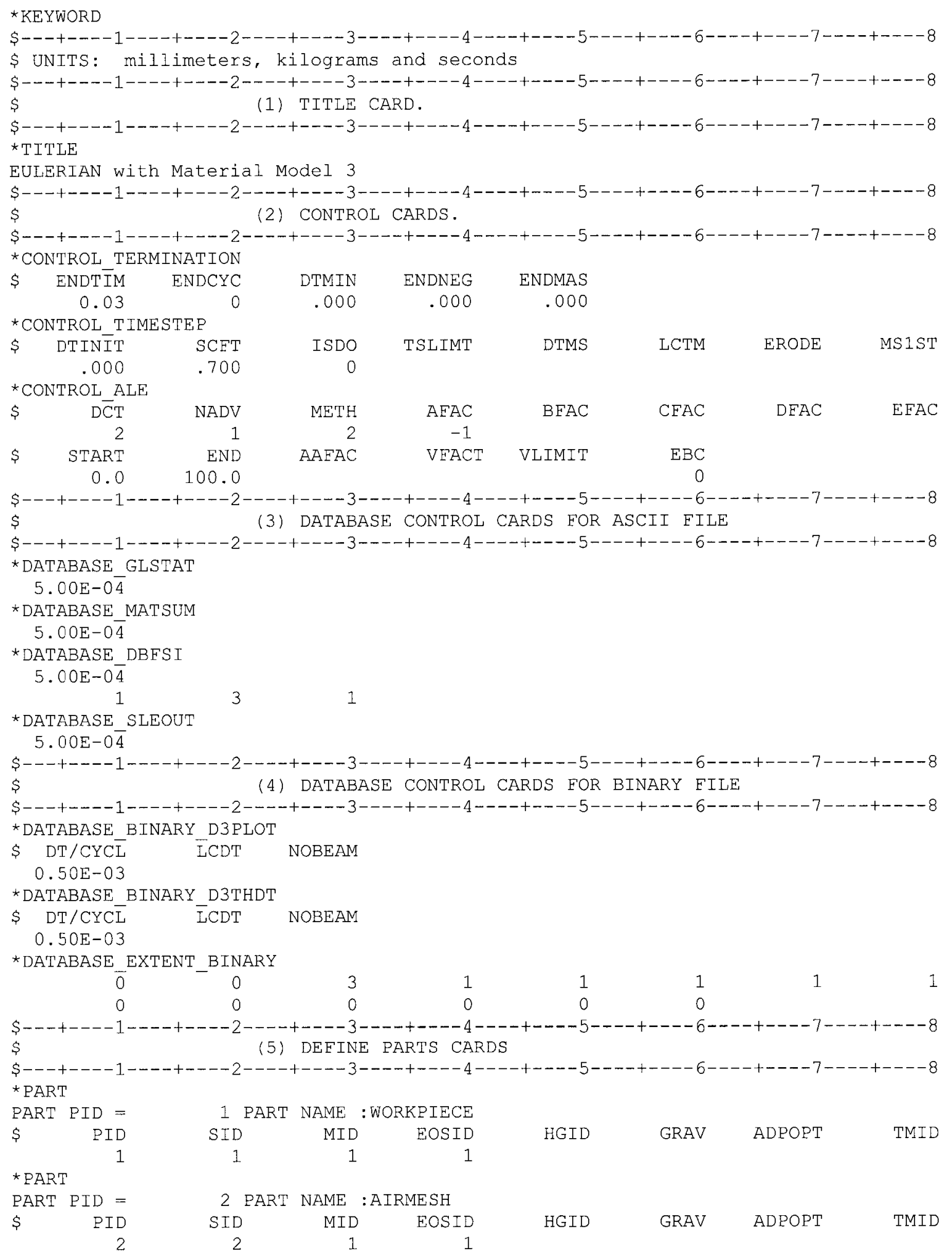


*PART

PART PID $=3$ PART NAME : CUTTER

PID
3

$\$---+----1----+----2----+----3----+----4----+----5----+----6----+----7----+----8$ $\$$ (8) E.O.S DEFINITIONS

$\$---+---1---+----2----+----3----+----4----+----5----+----6----+----7----+----8$ *EOS GRUNEISEN

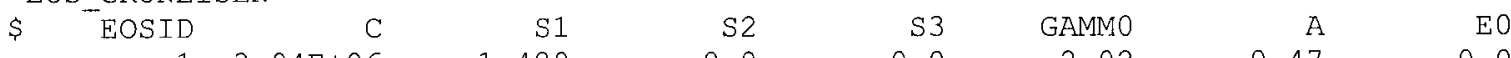
$\$ \quad \begin{array}{rrrrrrrr}1 & 3.94 \mathrm{E}+06 & 1.489 & 0.0 & 0.0 & 2.02 & 0.47 & 0.0\end{array}$

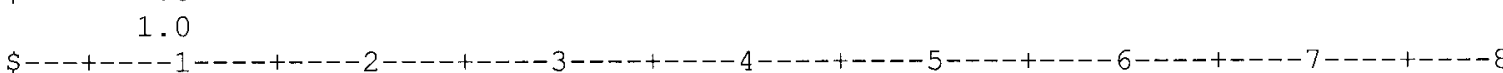

$\$$
$\$$
$\$--+---1---+---2-(9)$ SECTION CARDS 


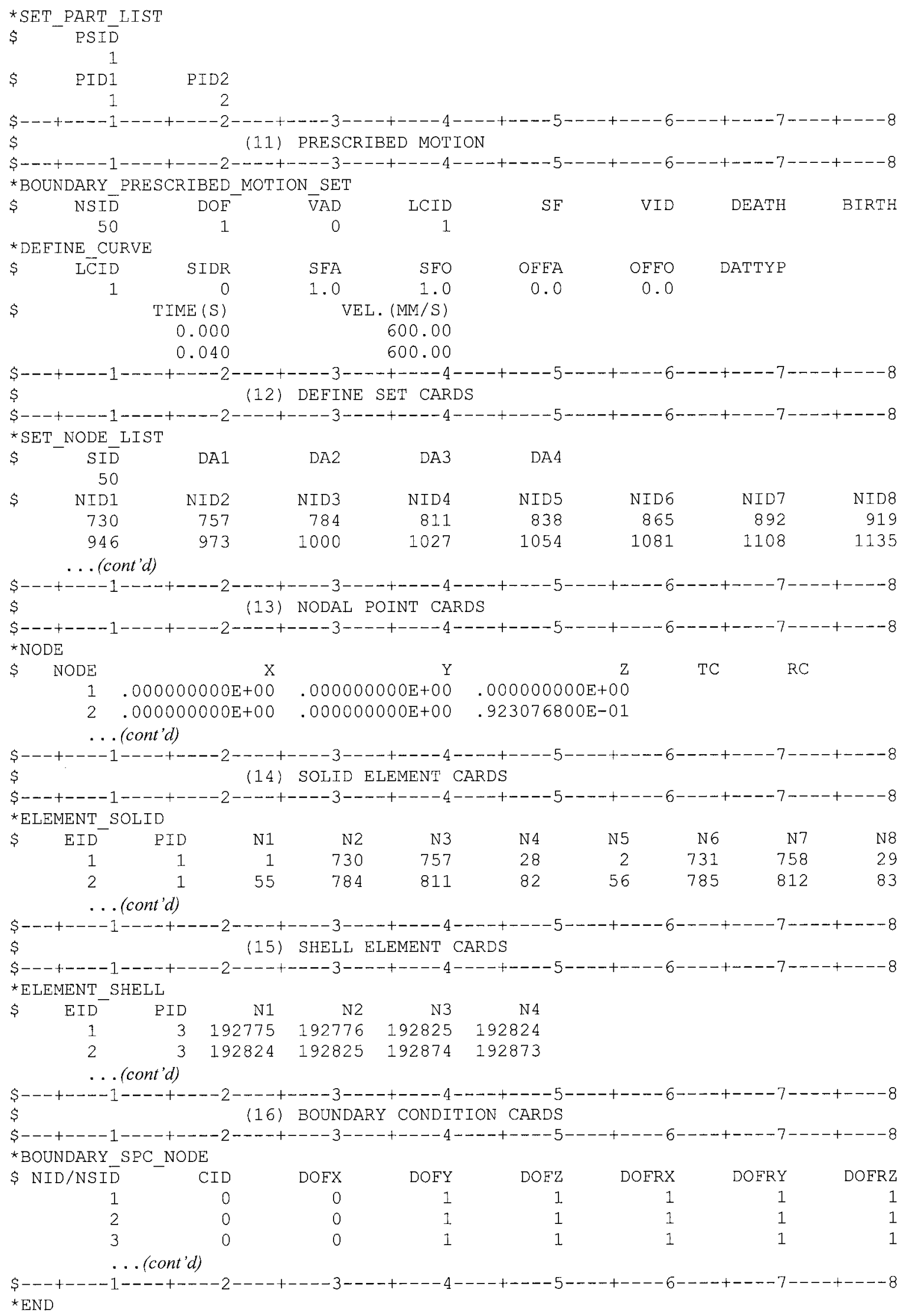




\section{APPENDIX K \\ Derivation of Johnson-Cook Parameters from experimental results}

In order to customize the Johnson-Cook parameters to the available experimental data in [56], the first term of the Johnson-Cook constitutive equation, expressed as Equation (A-28) was fitted to the experimental results.

$$
\sigma=A+B \varepsilon^{p "}
$$

By analyzing Equation (A-28) it can be established that the parameter $A$ is simply the stress corresponding to zero plastic strain or the yield stress $\left(\sigma_{\text {yield }}\right)$. Parameters $B$ and $n$ account for work hardening effects.

STEP 1 - Finding parameter A: The experimental stress-strain points and its corresponding Voce curve fit, found by Elmadagli and Alpas [56], are shown in Figure A.3.

From the Voce equation curve fit, the yield stress representing the experimental stress at zero experimental strain is 199.3 $\mathrm{MPa}$. Therefore $\mathbf{A}=199.3 \mathrm{MPa}$.

STEP 2 - Finding Parameters $B$ and $n$ : The log of equation (A-28) produces the following expression:

$$
\log (\sigma-A)=n \cdot \log \left(\varepsilon^{p}\right)+\log (B) .
$$

Equation (A-29) has the form of a linear relationship resembling:

$$
y=n \cdot x+b
$$

where $y=\log (\sigma-A), n$ is the slope of the experimental $\log -\log$ plot, $x=\log \left(\varepsilon^{p}\right)$, and $b=\log (B)$. Figure A.4 is the $\log \log$ plot of the data in Figure A.3, from the figure the curve fit to the experimental data in the form of Equation (A-29) is:

$$
\log (\sigma-A)=0.325 \cdot \log \left(\varepsilon^{p}\right)+\log (105.2) .
$$

Therefore parameter $B$ is $105.2 \mathrm{MPa}$ and the work hardening exponent $n$ is 0.325 . Recall that A is the yield strength of the material or $199.3 \mathrm{MPa}$, and the stress-strain relationship in Equation (A-28) for C11000 becomes:

$$
\sigma=199.3+105.2 \varepsilon^{p^{0.325}}
$$




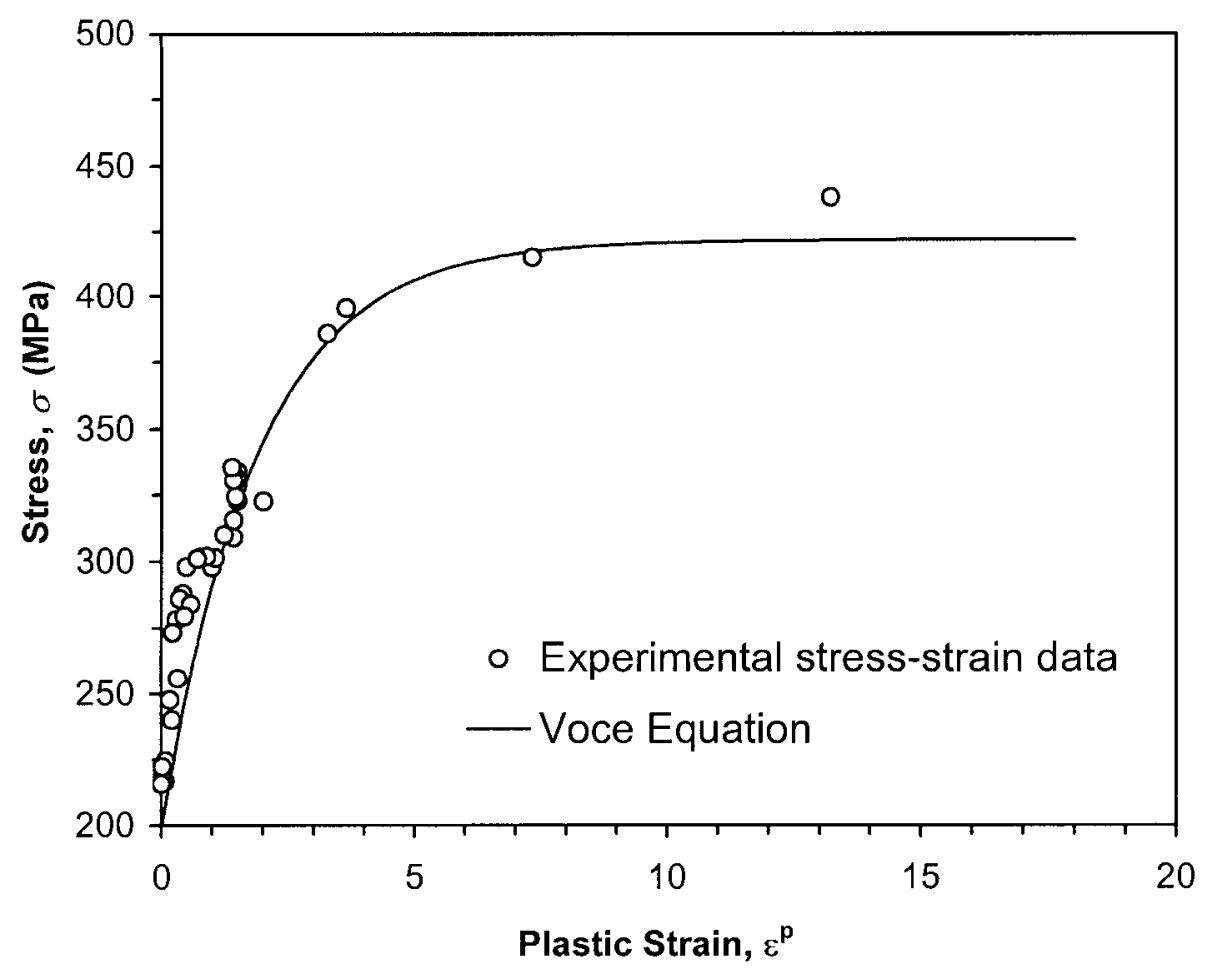

Figure A.3: Plot of experimental equivalent strain vs. flow stress [56]

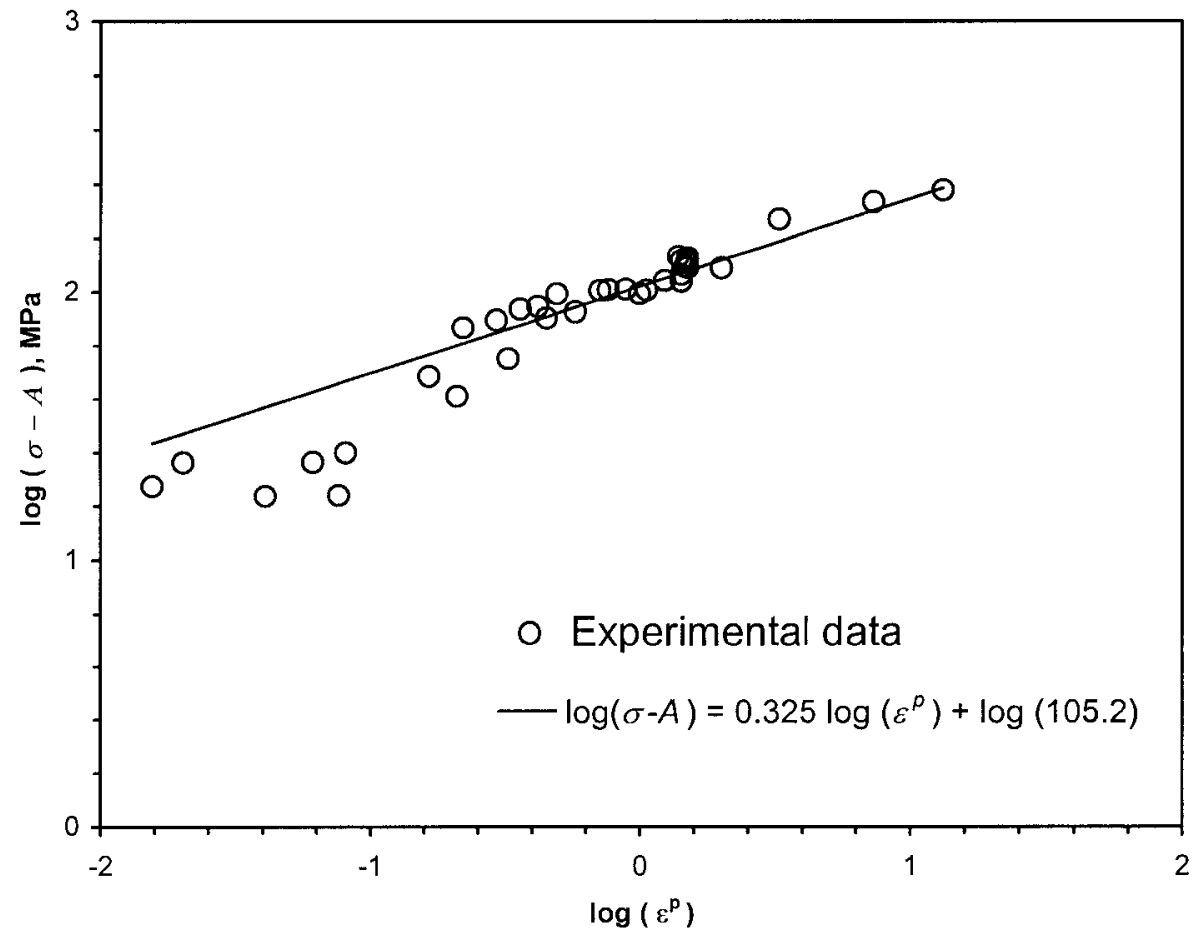

Figure A.4: Log-Log plot of the experimental stress-strain data, with a linear curve fit. 


\section{PUBLICATIONS}

1. Experimental Testing and Numerical Modeling of AM50A Magnesium Alloy for Structures Subjected to Large Deformation; Computational Methods and Experimental Measurements Conference; Spain, June 2001

2. Numerical Simulation of AM50A Magnesium Alloy Under Large Deformation; International Journal of Impact Engineering, vol. $30(2)$, pp. 117-142, February 2004.

3. An Eulerian Finite Element Model for Determination of Deformation State of a Copper Subjected to Orthogonal Cutting; Metallurgical and Materials Transactions A, accepted for publication, January 2004.

4. An Eulerian Finite Element Model of the Metal Cutting Process; $8^{\text {th }}$ International LS-DYNA Users Conference, Dearborn MI, submitted December 2003. 


\section{VITA AUCTORIS}

NAME:

Anna G. Raczy

PLACE OF BIRTH: Rzeszów, Poland

YEAR OF BIRTH: 1978

EDUCATION: $\quad$ University of Windsor, Windsor, ON

Department of Mechanical, Materials and Automotive Engineering M.A.Sc. in Engineering Materials

January 2002 - January 2004

University of Windsor, Windsor, ON

Department of Mechanical, Materials and Automotive Engineering B.A.Sc. in Mechanical Engineering (Materials Option)

September 1997 - August 2001 